\title{
Measurement of the Absolute Energy Scale of MINOS and Background Measurements in NEMO-3
}

\author{
Leo Jenner
}

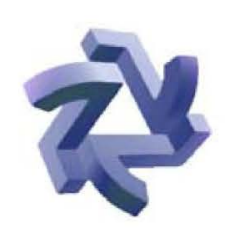

\section{NEMO-3}

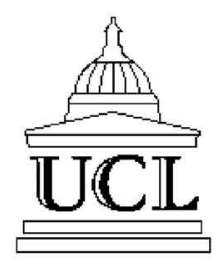

\section{University College London}

Submitted to the University of London in fulfilment of the requirements for the award of the degree of Doctor of Philosophy

September 2005 


\section{Abstract}

MINOS (Main Injector Neutrino Oscillation Search) is an experiment currently running in the US. A beam of neutrinos is created at Fermilab, Chicago, measured in the 1 kiloton 'Near Detector' and then travels 730km to the 5 kiloton 'Far Detector' in the Soudan Mine, Minnesota. In the intervening time, it is hoped that some of these neutrinos will change from one flavour to another. If this is observed, it is strong evidence for neutrino oscillations, the parameters of which can be measured to $10 \%$.

The MINOS experiment is a large project with a huge number of technical issues. Many aspects of the experiment were tested several years before the main experiment itself began to run, by employing a scaled down version of the detectors, known as the Calibration Detector (CalDet). This was placed in a test-beam at CERN and extensively studied, the data from which is analysed in this thesis.

In this thesis, photomultiplier tube crosstalk is discusseed, a phenomenon which generates false signals in the MINOS detectors. It is studied and an algorithm presented to enable its removal. Particle identification via various methods at CalDet is also described. Various pieces of hardware are available to assist with this, and a comparison is made to software techniques which are used at the larger MINOS detectors. A study of the CalDet beamline simulation is carried out and the discrepancies with data highlighted and explained. Finally, muon energy loss in CalDet is investigated. A comparison is made between published data and the observed data.

NEMO-3 is an experiment that has been ruming for some time in the Fréjus tunnel between France and Italy. It is a $0 \nu \beta \beta$ experiment, hoping to show that the neutrino is a Majorana particle and set limits on its mass.

This experiment, like every, has backgrounds. The dangerous background signals 
that arise from the radioactive decay of Uranium and Thorium are discussed in this thesis, specifically the measurement of the quantity of ${ }^{208} \mathrm{TI}$ and ${ }^{214} \mathrm{Bi}$ in the source foils of the detector. This is achieved by using Monte Carlo simulations of the contaminants behaviour in the detector, developing cuts on these events and applying them to the dataset. 


\section{Acknowledgements}

I would like to thank my supervisor, Jenny Thomas, for guidance, advice and persistence throughout my $\mathrm{PhD}$. I've met many people and gratefully received assistance from innumerable sources in London, Minnesota and Geneva, in particular from the old CalDet team: Phil Adamson, Anatael Cabrera, Jeff Hartnell, Mike Kordosky, Jason Koskinen, Karol Lang, Rob Morse, Ryan Nichol, Ruben Saakyan, Chris Smith, Jenny Thomas, Trisha Vahle and Alejandro, amongst others.

Whilst my involvement with the NEMO collaboration has been fairly minor, I'd like to thank Serge Julian, Dominique Lalanne and Ladislav Vala especially for welcoming me into the experiment.

On my journey through the PhD, there have always been people willing to lend a hand whenever I needed it and for whatever reason. My thanks go to Tristan Marshall, Atila Neves, Stuart Patterson and far too many others to mention here. Finally, I would like to thank my family, for continued support and encouragement throughout my student days. 


\section{Contents}

$\begin{array}{lll}1 & \text { Introduction } & 17\end{array}$

1.1 A Brief History of the Neutrino . . . . . . . . . . . . . 17

2 Neutrino Physics $\quad 20$

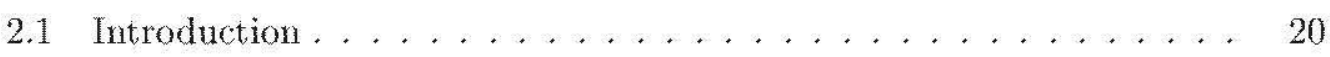

2.2 The Standard Model . . . . . . . . . . . . . . . . . 20

2.2 .1 Fermions . . . . . . . . . . . . . . . . 21

2.2 .2 Bosons. . . . . . . . . . . . . . . . . . . . . 22

2.3 Mass in the Standard Model . . . . . . . . . . . . . . . . . 23

2.4 Massive Neutrinos . . . . . . . . . . . . . . 27

2.5 Neutrinoless Double Beta Decay . . . . . . . . . . . . . 28

2.6 Neutrino Oscillations . . . . . . . . . . . . . . . 30

2.6 .1 MSW effect . . . . . . . . . . . . . . . . 34

3 The History of Neutrino Experiments $\quad 40$

3.1 Introduction . . . . . . . . . . . . . . . . 40 40

3.2 Measurements of Neutrino Masses . . . . . . . . . . . . . . . . 41

3.3 Direct Techniques. . . . . . . . . . . . . . . . . . . 42

3.4 Indirect Techniques . . . . . . . . . . . . . . 44

3.4.1 The Effective Majorana Mass of the Neutrino . . . . . . . 44

3.4.2 The Mass Difference Squared and mixing between neutrino species. . . . . . . . . . . . . . . . 4 48

3.5 Summary . . . . . . . . . . . . . . . . 59 
4 The NuMi-MINOS Experiment $\quad 65$

4.1 Introduction . . . . . . . . . . . . . . . 65

4.2 NuMI . . . . . . . . . . . . . . . . . 66

4.3 General Design of the MINOS detectors . . . . . . . . . . 68

4.4 The Near Detector . . . . . . . . . . . . . . . . 69

4.5 The Far Detector . . . . . . . . . . . . . . . 72

4.6 The Calibration Detector . . . . . . . . . . . 75

4.7 CERN Test Beams . . . . . . . . . . . . . . . . 79

4.8 MINOS Physics. . . . . . . . . . . . . . . . . . . . . 82

5 Calibration and Crosstalk $\quad 87$

5.1 The Calibration Chain . . . . . . . . . . . . . 87

5.1 .1 PS muons . . . . . . . . . . . . . . . . . 9 90

5.2 Crosstalk . . . . . . . . . . . . . . . . . . . 91

5.2 .1 Introduction . . . . . . . . . . . . . . . . 91

5.2 .2 Characteristics of Crosstalk in Data . . . . . . . 96

5.2 .3 Crosstalk Removal Algorithm . . . . . . . . . . . . . . . 104

5.2 .4 Algorithm Performance . . . . . . . . . . . . . . . 106

5.3 Summary . . . . . . . . . . . . . . . . . . . . 109

5.4 Pion/Muon Separation . . . . . . . . . . . . . . 110

5.5 Muon selection using the Cerenkov Detectors . . . . . . . . . 111

5.5 .1 Cerenkov efficiency . . . . . . . . . . . . . 114

5.6 Muon selection using shape and energy cuts . . . . . . . . . . 122

5.6 .1 Muon Spectrum ... . . . . . . . . . . . . 122

5.6 .2 Beamline Simulation . . . . . . . . . . . . 124

5.6 .3 Particle Definitions . . . . . . . . . . . . . . . 124

5.7 Measurement of Muon $d \mathrm{E} / d \mathrm{X} \ldots \ldots \ldots \ldots . \ldots . \ldots 140$

5.7 .1 Bethe-Bloch Fitting . . . . . . . . . . 145

5.8 Summary . . . . . . . . . . . . . . . 149

6 The NEMO-3 Experiment 152

6.1 Introduction . . . . . . . . . . . . . . . 152 
6.2 Detector Description . . . . . . . . . . . . . . 152

6.2 .1 Source Foils . . . . . . . . . . . . . . . . 154

6.2 .2 Tracking Wire Chamber . . . . . . . . . . . . 155

6.2 .3 Calorimeter . . . . . . . . . . . . . 155

6.2 .4 Electronics . . . . . . . . . . . . . 157

6.2 .5 Magnetic Field and Shielding . . . . . . . . . . . 159

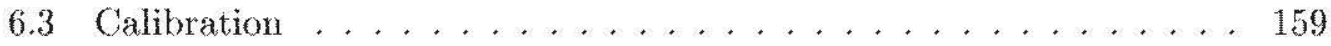

6.4 NEMO Physics . . . . . . . . . . . . . . . . . 160

7 Background studies in the NEMO-3 experiment 162

7.1 Backgrounds of the NEMO-3 experiment . . . . . . . . . . . 162

7.1 .1 The tail of the $2 \nu \beta \beta$ distribution . . . . . . . 163

7.1 .2 Neutrons and Photons . . . . . . . . . . . . . . . 163

7.1 .3 Radon . . . . . . . . . . . . . . . . . . . . . 164

$7.1 .4{ }^{214} \mathrm{Bi}$ and ${ }^{208} \mathrm{Tl} \ldots \ldots \ldots \ldots \ldots \ldots . \ldots \ldots 4 . \ldots \ldots$

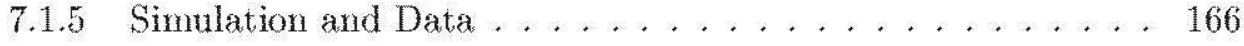

7.1 .6 Particle Definitions . . . . . . . . . . . . 166

7.1 .7 Selection Cuts ................... 167

7.1 .8 Calculation of Probability from $\chi^{2} \ldots \ldots \ldots 172$

7.1 .9 Calculation of Number of Events . . . . . . . 172

7.2 Measurement of the ${ }^{208} \mathrm{TI}$ Background in the Foils . . . . . . . 173

7.2 .1 Method ......................... 173

7.2 .2 Result . . . . . . . . . . . . . . . 177

7.3 Measurement of the ${ }^{214} \mathrm{Bi}$ Background in the Foils . . . . . . . 179

7.3 .1 Method . . . . . . . . . . . . . . 179

7.3 .2 Result . . . . . . . . . . . . . . . . . . . 181

7.4 Summary . . . . . . . . . . . . . . . 182

7.4 .1 Further Notes. . . . . . . . . . . . . . . . . 184

$\begin{array}{llr}8 \text { Conclusions } & 187\end{array}$

$\begin{array}{lr}\text { A Molybdenum Purification Processes } & 189\end{array}$

A.1 ${ }^{100}$ Mo Metallic Foils . . . . . . . . . . . . . . . . . . 189 
A.2 ${ }^{100}$ Mo Composite Foils . . . . . . . . . . . . . . . . . . 189 


\section{List of Figures}

2.1 The Higgs Potential . . . . . . . . . . . . . . . . 25

2.2 Modes of Double Beta Decay . . . . . . . . . . . . . 30

2.3 Double beta decay energy signatures . . . . . . . . . . . 31

2.4 Vacuum Oscillation probability ................. 33

2.5 Elastic Neutral Current Forward Scattering . . . . . . . . . . . 34

2.6 Elastic Charged Current Forward Scattering . . . . . . . . . 35

2.7 Matter Mixing Angle . . . . . . . . . . . . . . . 37

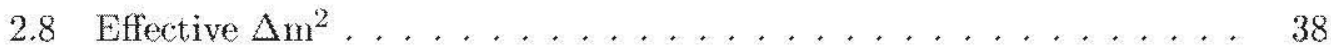

2.9 Oscillation Probability in the Earth . . . . . . . . . . 39

3.1 Kurie Plot of Tritium Decay . . . . . . . . . . . . . . . . . 43

3.2 Two neutrino and neutrinoless double beta decay . . . . . . . 45

3.3 Solar Neutrino Spectra . . . . . . . . . . . . . . . . . . 49

3.4 Neutrino interactions in normal and heavy water . . . . . . . 51

3.5 Comparison of Predicted Solar Fluxes to Experimental Measurements 52

3.6 Interactions from Cosmic Rays in the Atmosphere . . . . . . . 53

3.7 Atmosperic neutrino distance travelled verses zenith angle . . . . 55

3.8 Allowed and Excluded Regions of Parameter Space for $\nu_{\mu} \leftrightarrow \nu_{\tau}$ ascillations. . . . . . . . . . . . . . . . . . . 61

3.9 Allowed Parameter Space from Super-Kamiokande and K2K experi-

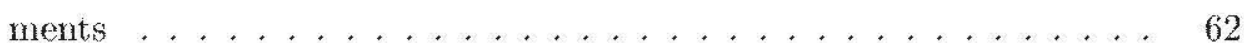

3.10 Allowed and Excluded Regions of Parameter Space for $\nu_{\mu} \leftrightarrow \nu_{e}$ oscillations. . . . . . . . . . . . . . . . . . 63

3.11 Allowed Parameter Space from KamLAND and solar experimetns. . 64 
4.1 Side view of the beam path . . . . . . . . . . 65

4.2 State map of the beam path . . . . . . . . . . 66

4.3 NuMI beamline at Fermilab . . . . . . . . . . . . 67

4.4 The three configurations of the NuMI beam . . . . . . . . 68

4.5 The instrumented regions of the Near Detector. . . . . . . . . 70

4.6 Partially and Fully Instrumented Near Detector Planes . . . . . . . 71

4.7 The Completed Near Detector . . . . . . . . . . . . 72

4.8 The Completed Far Detector . . . . . . . . . . . . . 73

4.9 Spot layout on PMT Pixels . . . . . . . . . . . . . 73

4.10 A CalDet Module . . . . . . . . . . . . . . . . 76

4.11 The Calibration Detector in the T11 testbeam area. . . . . . 77

4.12 The East Hall . . . . . . . . . . . . . . . . . . . . 80

4.13 Cerenkov detector at CalDet . . . . . . . . . . . 81

4.14 Cerenkov and TOF at CalDet . . . . . . . . . . . . . 81

4.15 Event Classes . . . . . . . . . . . . . . . . . 83

4.16 The $90 \%$ confidence limits on $\nu_{p t} \rightarrow \nu_{\tau}$ oscillation parameters . . 84

4.17 The oscillated and unoscillated charged current $\nu_{\mu}$ energy spectra. . 85

4.18 Limit plots for $\nu_{\mu} \rightarrow \nu_{a n y}$ from charged current $\nu_{\mu}$ interactions. . . . 85

4.19 Limit plots for $\nu_{p} \rightarrow \nu_{e} \ldots \ldots \ldots \ldots$. . . . . . 86

5.1 Light Injection Schematic . . . . . . . . . . . . . . . . 89

5.2 A Cosmic Ray Muon at CalDet . . . . . . . . . . . . . . . 92

5.3 A $1.8 \mathrm{GeV}$ beam muon . . . . . . . . . . . . . . 93

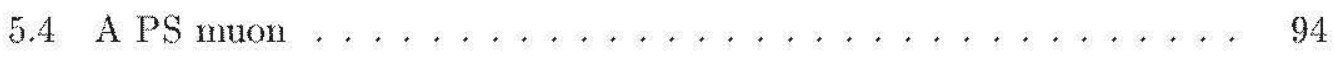

5.5 Photomultiplication method .................. 95

5.6 An M16 PMT . . . . . . . . . . . . . . . . . . . . . 95

5.7 Pixel numbering on PMT faces and spot positions . . . . . . . 97

5.8 Tracking before and after crosstalk removal . . . . . . . . . 98

5.9 Quantity of crosstalk to neighbouring pixels showing percentages of crosstalk to other pixels ... . . . . . . . . . . . . 99

5.10 Event display with hits on PMT face shown for a selected region . . 100

5.11 Quantity of crosstalk to neighbouring pixels showing number of entries 101 
5.12 Hit ADC vs Crosstalk ADC . . . . . . . . . . . . . . 102

5.13 Hit ADC vs crosstalk ADC with $\mathrm{x}$ and y projections . . . . . . 103

5.14 Number of crosstalk hits compared to real hits . . . . . . . . . 104

5.15 Crosstalk ADC/Source ADC . . . . . . . . . . . . . 105

5.16 Quantity of crosstalk identified . . . . . . . . . . . 107

5.17 Muon ranges with and without crosstalk removal . . . . . . . 108

5.18 Cerenkov Pressure Thresholds . . . . . . . . . . . . . . . 112

5.19 Raw events separated by the cerenkov counters at 1.8 and $2.0 \mathrm{GeV} .113$

5.20 Range verses cerenkov adc . . . . . . . . . . . . . . . 115

5.21 Effect of fiducial and range cuts . . . . . . . . . . . 116

5.22 Range verses $\beta$ for muons and pions . . . . . . . . . 117

5.23 Muon Detection Efficiency verses Plane . . . . . . . . . . . . 118

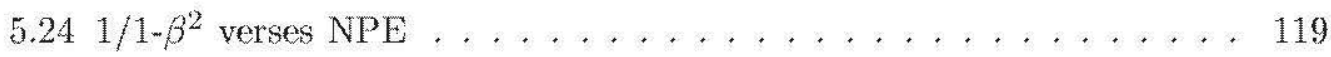

$5.25 \chi^{2}$ fits to number of points and determination of the cerenkov thresholds 120

5.26 The distribution of muon momenta at $1.8 \mathrm{GeV} \ldots \ldots . . . . . .121$

5.27 Corrected plane-by-plane efficiencies . . . . . . . . . . . . 121

5.28 Fits to range plots . . . . . . . . . . . . . . . . 122

5.29 Corrected range plots . . . . . . . . . . . . . . . 123

$5.30 \mathrm{~T} 11$ Muon Monte Carlo . . . . . . . . . . . . . . . . . . . . 125

5.31 T7 Muon Monte Carlo - Linear . . . . . . . . . . . . . . . 126

5.32 T7 Muon Monte Carlo - Log . . . . . . . . . . . . . 127

5.33 GEANT $1.8 \mathrm{GeV}$ muons with and without beamline simulation . . 128

5.34 TOF time differences . . . . . . . . . . . . . . . 131

5.35 Multiplicity and E3 cut distributions . . . . . . . . . 132

5.36 Selection efficiencies for pions and muons $\ldots \ldots \ldots 133$

5.37 Beam Composition . . . . . . . . . . . . . . . . 134

5.38 GEANT pion decay vertices . . . . . . . . . . . 135

5.39 Shape/energy selected muons that fire the upstream cerenkov . . . 136

5.40 Range components of the complete Monte Carlo muon spectrum . . 138

5.41 FractionFitter results for largest collimator aperture . . . . . . . 139

5.42 FractionFitter results for smallest collimator aperture . . . . . 140 
$5.43 \chi^{2} /$ n. d.f. for various energy shifts . . . . . . . . . . . 141

5.44 Bethe-Bloch formula for muons travelling through Copper. . . . . 142

$5.451 .8 \mathrm{GeV}$ Plane Hit Distributions . . . . . . . . . . . 144

$5.46 \mathrm{dE} / \mathrm{dX}$ fitting with nominal parameters . . . . . . . . 146

5.47 Parameters extracted from $\mathrm{dE} / \mathrm{dX}$ fitting $\ldots \ldots \ldots . . \ldots 147$

5.48 Bethe-Bloch fits at $1.8,3$ and $10 \mathrm{GeV} \ldots \ldots \ldots . \ldots 148$

5.49 Comparison of $\mathrm{dE} / \mathrm{dX}$, data and theory . . . . . . . 150

6.1 The NEMO-3 'Camembert' . . . . . . . . . . . . . . . . 153

6.2 The Structure of the NEMO-3 Geiger Wire Chamber . . . . . . . 156

6.3 The Structure of NEMO-3 Sectors . . . . . . . . . . . . . 158

$6.4 \beta \beta$ event display $\ldots \ldots \ldots \ldots \ldots \ldots \ldots \ldots$

$7.1{ }^{238} \mathrm{U}$ and ${ }^{232} \mathrm{Th}$ decay chains $\ldots \ldots \ldots \ldots . \ldots . \ldots . \ldots 5$

7.2 Probability distribution of internal electron hypothesis . . . . . 170

7.3 Probability distribution of external photon hypothesis . . . . . 170

7.4 Probability distribution of internal photon hypothesis . . . . . 171

7.5 Probability distribution of photon reflection hypothesis . . . . . 171

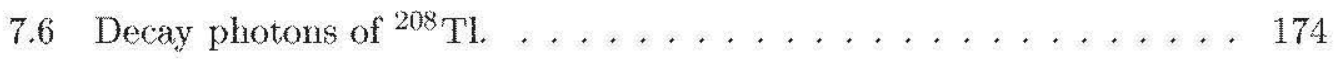

7.7 Principle events quantities of ${ }^{208} \mathrm{Tl}$ Monte Carlo decays. . . . . . . 176

$7.8{ }^{208} \mathrm{Tl}$ spectrum $\ldots \ldots \ldots \ldots \ldots \ldots \ldots$

$7.9{ }^{214}$ Bi event display - top view . . . . . . . . . . . . 179

$7.10{ }^{214} \mathrm{Bi}$ event display - side view . . . . . . . . . . . . 180

7.11 Decays of ${ }^{214} \mathrm{Bi} \ldots \ldots \ldots \ldots \ldots . \ldots \ldots$

7.12 Principle events quantities of ${ }^{214} \mathrm{Bi}$ Monte Carlo decays. . . . . . . 184

$7.13^{214}$ Bi spectra . . . . . . . . . . . . . . . . . 185

7.14 Half-life reconstruction . . . . . . . . . . . . . . 185 


\section{List of Tables}

2.1 Properties of Leptons . . . . . . . . . . . . . . . . . . 21

3.1 Direct Neutrino Mass Measurement . . . . . . . . . . . . 59

3.2 Mass Measurements from $0 \nu \beta \beta \ldots \ldots \ldots . . \ldots . . \ldots 59$

3.3 Neutrino Oscillation Parameters From Experiment . . . . . . . 60

4.1 Near Detector schematic . . . . . . . . . . . . . . . 70

4.2 Beam Line, Angle and Fibre Configurations of the CalDet Runs . . . 78

5.1 Pixel to Spot Configuration at CalDet . . . . . . . . . . 97

5.2 Cerenkov Efficiencies . . . . . . . . . . . . . 116

5.3 Fractions for various collimator settings $\ldots \ldots \ldots 136$

5.4 Fractions for various collimator settings . . . . . . . . . . 137

5.5 Progression of Monte Carlo simulation . . . . . . . . . . . 138

5.6 Muon Energy Loss Mechanisms in Iron . . . . . . . . . . . . . . 143

5.7 Muon Energy Loss Mechanisms in Polystyrene . . . . . . . . . . 145

5.8 Energy losses in Plane Components . . . . . . . . . . . . 146

5.9 Bethe-Bloch Fit Parameters . . . . . . . . . . . . 147

6.1 Nemo Source Foil Information . . . . . . . . . . . . . . . . . 154

7.1 The activities of the principle detector elements of NEMO-3 . . . 166

7.2 Decay Transition Energies of various ${ }^{208} \mathrm{Tl}$ Event Types . . . . . 173

7.3 Selection cuts applied to ${ }^{208} \mathrm{Tl}$ Monte Carlo. . . . . . . . . . . . 177

7.4 Calculated Activities in the 1,2 and $3 \gamma^{208} \mathrm{Tl}$ Channel . . . . . 178

7.5 Decay Transition Energies of various ${ }^{214} \mathrm{Bi}$ decays . . . . . . . . . 181 
7.6 Monte Carlo ${ }^{214} \mathrm{Bi}$ selection cuts . . . . . . . . . . . 182

7.7 Calculated Activity for ${ }^{214} \mathrm{Bi} \ldots \ldots \ldots \ldots . \ldots \ldots 2$ 


\title{
List of Acronyms
}

\author{
ADC Analogue-to-Digital Conversion. \\ CalDet MINOS Calibration Detector. \\ CC Charged Current. \\ CERN Centre European de Recherche Nucleaire. \\ DAQ Data Acquisition. \\ FarDet MINOS Far Detector. \\ HV High Voltage. \\ HPGe High Purity Germanium (detector). \\ LEP Large electron-positron (collider).
}

LI Light Injection.

LSM Laboratoire Souterrain de Modane.

M16 16 pixel PMT.

M64 64 pixel PMT.

MINOS Main Injector Neutrino Oscillation Search.

MIPP Main Injector Particle Production.

MSW Mikheyev-Smirnov-Wolfenstein. 
NearDet MINOS Near Detector.

NC Neutral Current.

NEMO Neutrino Ettore Majorana Observatory.

NuMI Neutrinos at the Main Injector.

PIN P-intrinsic-N (diode).

PMT / PM Photomultiplier tube.

PS Proton Synchrotron.

QIE Charge Integrator Encoder.

TDC Time-to-Digital Conversion.

TOF Time of Flight.

TPC Time Projection Chamber.

WLS Wavelength Shifting.

V-A Vector minus Axial.

VA Viking Analogue.

VARC VA Readout Card.

VME VERSAmodule Eurocard. 


\section{Chapter 1}

\section{Introduction}

\subsection{A Brief History of the Neutrino}

- 1896: Becquerel observes that uranium minerals emit radiations that can be 'recorded' on photographic emulsions. In this process, elementary particles are created apparently from nowhere and chemical elements spontaneously transform from one to another.

- 1930: $\alpha$-particles and $\gamma$-rays are understood to some extent, but $\beta$-rays appear to exhibit non-conservation of energy. Pauli proposes the existence of the 'neutron' in a drastic attempt to solve the problem [1].

- 1932: Chadwick discovers the neutron as it is known today, but it is too heavy to solve the $\beta$-ray problem [2].

- 1933: Fermi builds the theory of $\beta$-decay and the weak interaction around Pauli's hypothesis, naming the illusive particle the 'neutrino' [3].

- 1935: Maria Goeppert-Mayer predicts the existence of the two neutrino double beta decay process [4].

- 1939: W. H. Furry proposes neutrinoless double beta decay, based on Majorana's idea that a particle could be its own anti-particle [5].

- 1956: Frederick Reines and Clyde Cowan make first experimental detection of the neutrino by observing neutrinos from a nuclear reactor interacting with 
a mixture of water and cadmium chloride [6].

- 1957: Madame Wu shows that the weak interaction is 'left-handed' by observing that $\beta$-particles are preferentially emitted in a direction correlated with the nuclear spin $[7]$.

- 1957: Bruno Pontecorvo postulates that if different types of neutrino exist, they might be able to 'oscillate' from one type to another [8].

- 1961: The $\nu_{p}$ is first experimentally observed [9].

- 1968: Ray Davis makes the first experimental measurement suggesting a discrepancy between the number of $\nu_{e}$ neutrinos emitted by the sun and the number arriving at the earth [10].

- 1983: Atmospheric $\nu_{\mu}$ deficiency first observed $[11,12]$.

- 1987: 19 neutrinos observed from supernova SN1987A [11, 12].

- 1991: LEP shows that there are only 3 active neutrino species $[13,14,15,16]$.

- 2000: The $\nu_{\tau}$ is first experimentally observed [17].

- 2003: SNO shows that the total number of neutrinos arriving at the earth from the sun is in agreement with stellar models [18].

- 2004: Super-K data supports neutrino oscillations by observing a zenith angle dependence of $\nu_{\mu}$ deficit [19].

Today, there is extremely strong evidence to suggest that neutrinos have non-zero mass and that they can oscillate from one flavour to another.

The questions that remain today are:

- What the absolute values of their masses?

- What the values of the oscillation parameters?

Two experiments that are in operation today are addressing these questions. NEMO-3 is a neutrinoless double beta decay $(0 \nu \beta \beta)$ experiment that is hoping to 
find or improve the limits on the mass of the neutrino. MINOS is a long-baseline neutrino oscillation experiment that hopes to measure oscillation parameters to better than $10 \%$. 


\section{Chapter 2}

\section{Neutrino Physics}

\subsection{Introduction}

The neutrino is one small component of the theory known as the Standard Model $[20,21]$. It is the theory that describes all of the particles in nature and their interactions with each other. It is an incredibly successful theory having made predictions that were proven by experiment and having stood up to rigorous scientific testing. However, many believe that the Standard Model is an incomplete theory on account of the fact that many fundamental parameters are arbitrarily set and do not naturally evolve from the theory itself. Allowing massive neutrinos also requires an extension to the existing theory.

\subsection{The Standard Model}

According to the Standard model, there are two types of fundamental particles: fermions and bosons. Fermions are the constituents of matter and bosons are the force carrying particles. The three forces by which these particles interact are the Strong force, the Weak force and Electromagnetism. The force of Gravity is not included in the Standard Model. 


\subsubsection{Fermions}

All fermions have 1/2-integer values for their spin quantum number. They can be subdivided into quarks which can interact with other particles via all three forces and leptons which do not. Each of the two groups of particles have six species, plus their antiparticle partners. It is convenient to further separate the leptons and quarks into generations which are indicative of their mass hierarchies. Within the lepton sector, the first generation doublet is therefore composed of the negatively charged electron $\left(\mathrm{e}^{-}\right)$and an electron neutrino $\left(\nu_{e}\right)$. The second generation consists of the muon $(\mu)$ and the muon neutrino $\left(\nu_{p}\right)$ and the third generation comprises the tau $(\tau)$ and the tau neutrino $(\tau)$. Muons and taus are unstable particles that decay into lighter charged leptons, but always accompanied by their counterpart neutrino. Neutrinos are only able to interact with other particles via the Weak Force, whilst charged leptons can also interact via Electromagnetism.

$$
\text { Leptons: }\left(\begin{array}{l}
\nu_{e} \\
e^{-}
\end{array}\right) \quad\left(\begin{array}{l}
\nu_{\mu} \\
\mu^{-}
\end{array}\right) \quad\left(\begin{array}{l}
\nu_{\tau} \\
\tau^{-}
\end{array}\right)
$$

\begin{tabular}{|c|c|c|c|c|}
\hline Gen. & Flavour & $\begin{array}{c}\text { Charge } \\
(\mathrm{Q})\end{array}$ & $\begin{array}{c}\text { Mass } \\
(\mathrm{MeV})\end{array}$ & Lifetime \\
\hline \multirow{2}{*}{1} & $\mathrm{e}$ & -1 & 0.51 & $>4.2 \times 10^{24} \mathrm{yr}$ \\
& $\nu_{e}$ & 0 & $<3 \times 10^{-6}$ & $>21 \times 10^{9} \mathrm{~s}$ \\
\hline 2 & $\mu$ & -1 & 105.7 & $2.2 \times 10^{-6} \mathrm{~s}$ \\
& $\nu_{\mu}$ & 0 & $<0.19$ & $>2.9 \mathrm{~s}$ \\
\hline 3 & $\tau$ & -1 & 1777 & $291 \times 10^{-15} \mathrm{~S}$ \\
\hline
\end{tabular}

Table 2.1: Some properties of the known leptons [21].

All quarks carry an electromagnetic charge that is some fraction of the absolute charge on the electron: the up (u), charm (c) and top (t) quarks possessing $+2 / 3$, and down (d), strange (s) and bottom (b) quarks carrying -1/3. They can be arranged into generations as follows: 


$$
\text { Quarks: }\left(\begin{array}{l}
u \\
d
\end{array}\right) \quad\left(\begin{array}{l}
c \\
s
\end{array}\right) \quad\left(\begin{array}{l}
t \\
b
\end{array}\right)
$$

Quarks also carry the colour 'charge' associated with the strong force. Quarks can be red, green or blue with anti-quarks being anti-red, anti-green or anti-blue. A property of the strong force is that quarks cannot exist individually. In nature they form hadrons which are colour-neutral collections of two or three quarks that carry integer or zero electromagnetic charge. Hadrons formed by three quarks, each with a different colour, are known as baryons. Common examples of baryons are the proton (uud) and the neutron (udd). Two-quark hadrons, one being a quark and the other being an anti-quark, are known as mesons, an example being the the $\pi^{-}$ $(\bar{u} \mathrm{~d})$.

\subsubsection{Bosons}

Bosons have integer or zero values of spin. Bosons act as force carriers that permit interactions between particles, each force having one or more bosons associated with it. The properties of the boson itself are an important part of the manifestation of the force; for example the range of the force is inversely proportional to the mass of the boson.

The Electromagnetic force is the simplest in many ways, with a single, massless, chargeless boson as the force carrier: the photon. The range of this force is infinite and it is only felt by particles that carry an electric charge.

The Strong force is mediated by eight massless, chargeless but coloured bosons known as gluons. Unlike photons, gluons can couple to each other and the force between them increases with their separation. When they become too far apart, the potential energy in the bond between them creates quark-anti-quark pairs from the vacuum. This prevents quarks existing in isolation and also effectively reduces the strong force's range despite the gluons being massless.

The Weak force is of most interest to neutrino physics since it is the only force by which the neutrino can interact. It is carried by three massive bosons, the $\mathrm{W}^{+}$, $W^{-}$and the $Z^{0}$. The $W^{ \pm}$bosons are electromagnetically charged and have masses 
of $80.45 \mathrm{GeV}$. The $\mathrm{Z}^{0}$ has a mass of $91.2 \mathrm{GeV}$ and no charge. The high masses of the weak bosons cause the force to be very short ranged.

Weak interactions have a strange property in that they violate parity. It was indirectly observed that neutrinos produced from $\beta$-transitions of magnetically polarized cobalt nuclei $[7]$ in the reaction:

$$
{ }^{60} \mathrm{Co} \rightarrow{ }^{60} N i^{*}+e^{-}+\bar{\nu}_{e}
$$

preferentially travelled in the opposite direction to that of the nuclear spin of the cobalt. This implied that only left-handed chiral fermions and right-handed chiral antifermions participated in weak interactions. In the case of massless neutrinos, the left-handed chiral states are identical to the observable left-handed helicity states. For Dirac particles, the mass term:

$$
-m \bar{\psi} \psi=-m\left(\bar{\psi}_{R}+\bar{\psi}_{L}\right)\left(\psi_{R}+\psi_{L}\right)=-m\left(\bar{\psi}_{R} \psi_{L}+\bar{\psi}_{L} \psi_{R}\right)
$$

always connects the opposite chiral components of the same field. Thus the absence of either $\psi_{R}$ or $\psi_{L}$ automatically leads to $\mathrm{m}=0$. With no right-handed neutrinos observed, they were assumed not to exist and this lead to neutrino masses being defined as zero within the standard model, although extremely small neutrino masses $(\sim$ few $\mathrm{eV})$ are still consistent with the observed parity violation.

\subsection{Mass in the Standard Model}

The standard model is a $\mathrm{SU}(3) \otimes \mathrm{SU}(2) \otimes \mathrm{U}(1)$ gauge theory [22]. In quantum field theory, fermions are described in terms of Lagrangians and field equations. When a local gauge symmetry is imposed on a fermion field, a conserved quantity can be defined. In this case, the conserved quantity is the charge associated with the force involved. If we take for example the Lagrangian [23] of a free fermion:

$$
\mathcal{L}=\bar{\psi}\left(i \gamma^{\mu} \partial_{\mu}-m\right) \psi
$$

and local U(1) symmetry of the form: 


$$
\psi \rightarrow \psi^{6}=e^{i \alpha(x) Q} \psi
$$

is imposed, the Lagrangian for quantum electodynamics (QED) is obtained:

$$
\mathcal{L}=\bar{\psi}\left(i \gamma^{\mu} \partial_{\mu}-m\right) \psi-e \bar{\psi} \gamma^{\mu} Q \psi A^{\mu}-\frac{1}{4} F_{\mu \nu} F^{\mu \nu}
$$

where $A^{\mu}$ is the field required to preserve the invariance and $\mathrm{F}_{\mu \nu}=\partial^{\mu} A^{\nu}-\partial^{\nu} A^{\mu}$. For local gauge invariance the interaction term $-e \bar{\psi} \gamma^{\mu} Q \psi$ between the fermion field, $\psi$, and the photon field $A^{\mu}$ is necessary. $Q$ is the charge operator whose eigenvalues are conserved quantities; in this case, electromagnetic charge.

The electromagnetic and weak forces are unified in electroweak theory by the imposition of $\mathrm{SU}(2)^{L} \otimes \mathrm{U}(1)$ symmetry. If one requires this gauge invariance, four fields are introduced adding the following interaction terms to the Lagrangian:

$$
-g \bar{\chi}_{L} \gamma_{\mu} \mathbf{T} \cdot \mathbf{W}^{\mu} \chi_{L}=g^{i} \bar{\psi} \gamma_{\mu} \frac{Y}{2} \psi B^{\mu}
$$

where $\mathbf{W}^{\mu}$ and $\mathrm{B}^{\mu}$ are the vector fields introduced to preserve the gauge invariance. $\mathbf{T}$ and $\mathrm{Y}$ are the operators, the eigenvalues of which are conserved quantities governing the strength of the coupling.

We know the $W^{ \pm}$bosons only couple with left-handed fermions, therefore the fermion fields must be separated into left and right chiral projections:

$$
\begin{array}{ll}
\chi_{L}^{l}=\left(\begin{array}{c}
\nu_{e} \\
e^{-}
\end{array}\right)_{L} & \chi_{L}^{q}=\left(\begin{array}{l}
u \\
d
\end{array}\right)_{L} \\
\psi_{R}=e_{R}^{-} & \psi_{R}=u_{R}, d_{R}
\end{array}
$$

There is no right-handed neutrino state in the standard model.

Although we have four fields represented by four gauge bosons, unfortunately they are not the observed $W^{ \pm}, Z^{0}$ and $\gamma$. Due to the requirements of gauge invariance and theory renormalization, the four bosons must be massless. A separate process is required to obtain the familiar bosons and to generate mass for the $W^{ \pm}$and $\mathrm{Z}$ bosons. This process is known as the Higgs Mechanism [24]. 
Underlying the Higgs mechanism is the concept of spontaneous symmetry breaking. If we consider the four scalar particles, $\phi_{i}$, with a $\mathrm{SU}(2) \otimes \mathrm{U}(1)$ gauge invariant Lagrangian given by:

$$
\mathcal{L}=\left|\left(i \partial_{\mu}-g \mathbf{T} \cdot \mathbf{W}_{\mu}-g^{g} \frac{Y}{2} \psi B_{\mu}\right) \phi\right|^{2}
$$

It is necessary now to make the assumption that the vacuum is not a singlet of the gauge symmetry but rather that there are an infinite number of states with the same ground-state energy. The process of choosing one of these states is known as Spontaneous Symmetry Breaking as after this choice U(1) transformations can result in a different lowest energy state. Through this process fields obtain non-zero vacuum expectation values. The Higgs potential is an example of this and can be expressed mathematically as:

$$
V(\phi)=\frac{1}{2} \mu^{2} \phi^{2}+\frac{1}{4} \lambda \phi^{4}
$$

with $\mu^{2}<0$ and $\lambda>0$.

If this term is added to the Lagrangian, gauge invariance is preserved but the scalar fields acquire a non-zero vacuum expectation value. It also introduces an extra degree of freedom for each field resulting from the degeneracy of the vacuum state, often expressed as a Goldstone boson [25].

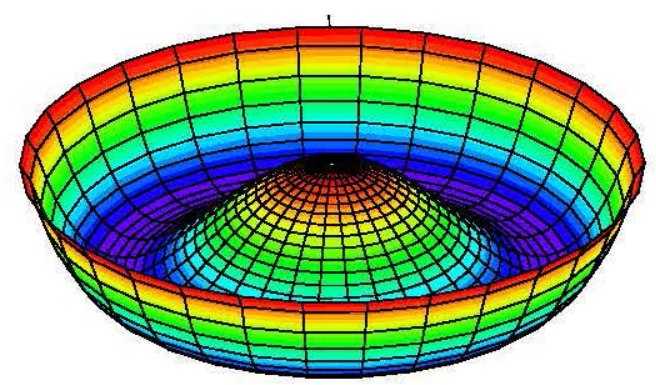

The Higgs Potential: $V(\phi)=\frac{1}{2} \mu^{2} \phi^{2}+\frac{1}{4} \lambda \phi^{4}$

Figure 2.1: Showing the Higgs Potential in 3 Dimensions.

The Higgs scalar fields can be defined as an SU(2) doublet: 


$$
\phi=\left(\begin{array}{c}
\phi^{+} \\
\phi^{0}
\end{array}\right)=\frac{1}{\sqrt{2}}\left(\begin{array}{c}
\phi_{1}+i \phi_{2} \\
\phi_{3}+i \phi_{4}
\end{array}\right)
$$

The symmetry is broken by choosing one true vacuum state:

$$
\phi_{0}=\frac{1}{\sqrt{2}}\left(\begin{array}{l}
0 \\
\nu
\end{array}\right)
$$

with $\nu=\sqrt{-\mu^{2} / \lambda}$.

Once this choice is made the gauge symmetry is spontaneously broken and the vector fields $\mathbf{W}$ and $B$ become massive. The extra degrees of freedom due to the four degenerate vacua of the $\phi$ fields become the longitudinal polarizations for the $\mathbf{W}$ and $B$ fields.

The $W_{\mu}^{1}$ and $W_{\mu}^{2}$ fields now correspond to the massive $W_{p}^{+}$and $W_{\mu}^{-}$gauge fields. The physical $Z_{p}$ and $A_{p}$ fields are obtained from a mixing of the $W_{\mu}^{3}$ and $B_{\mu}$ :

$$
\begin{aligned}
& Z^{\mu}=\cos \theta_{w} W_{p}^{3}-\sin \theta_{w} B_{\mu} \\
& A^{\mu}=\sin \theta_{w} W_{\mu}^{3}+\cos \theta_{w} B_{\mu}
\end{aligned}
$$

where $\cos \theta_{w}$ is known as the Weinberg or weak mixing angle and can be expressed as the ratio of the $Z^{0}$ and $W^{ \pm}$masses $[26]$ :

$$
\cos \theta_{w}=\frac{M_{W}}{M_{Z}} \simeq 0.87679
$$

Although the $\mathrm{W}$ and $\mathrm{B}$ fields are massive, $\mathrm{A}_{\mu}$ is chosen such that the operator associated with it is $\mathrm{Q}=\mathrm{T}^{3}+\mathrm{Y} / 2$ and $\mathrm{Q}_{\phi_{0}}=0$. Therefore, spontaneous symmetry breaking generates mass for the $\mathrm{W}^{ \pm}$and $\mathrm{Z}$ bosons but the photon remains massless.

The Higgs scalar fields were introduced to generate mass for the $\mathrm{W}^{ \pm}$and the $\mathrm{Z}$ bosons but we can also consider its interaction with the fermion fields. It is possible to write down the Lagrangian for the Higgs-fermion couplings, which connect the left-handed doublet to the right-handed singlet fermion fields. For the first generation of leptons and quarks:

$$
\mathcal{L}=f^{e} \bar{\chi}_{L}^{l} \phi_{0} e_{R}+f^{u} \bar{\chi}_{L}^{q} \bar{\phi}_{0} u_{R}+f^{d} \bar{\chi}_{L}^{q} \phi_{0} d_{R}+h . c
$$


where $\bar{\phi}=i \tau_{2} \phi_{0}^{*}$, and $f^{x}$ are the Yukawa coupling constants for the $x$-Higgs interactions.

With equation 2.11 , symmetry breaking gives:

$$
\mathcal{L}=f^{e} \frac{v}{\sqrt{2}} \overline{e_{L}} e_{R}+f^{u} \frac{v}{\sqrt{2}} \overline{u_{L}} u_{R}+f^{d} \frac{v}{\sqrt{2}} \overline{d_{L}} d_{R}+h \cdot c
$$

Therefore, all of the fermions have acquired a mass of $\mathrm{f}^{x} \frac{\nu}{\sqrt{2}}$ except the neutrino.

\subsection{Massive Neutrinos}

The standard model requires an extension to incorporate massive neutrinos, but it is only a minor modification. The mass terms described previously have been Dirac mass terms, which couple left- and right-handed fields together. Dirac mass terms are the only kind available to charged particles. However neutrinos, being neutral, also have Majorana mass terms available to them. These couple particles to their anti-particles.

Defining the following conventions for the charge-conjugation operator, $C$, and the chiral fields, $\psi_{L}$ and $\psi_{R}$ :

$$
\begin{gathered}
\psi^{C}=C \gamma^{0} \psi^{*}=i \gamma^{2} \psi^{*} \\
\psi_{L}=\frac{1}{2}\left(1-\gamma_{5}\right) \psi, \quad \psi_{R}=\frac{1}{2}\left(1+\gamma_{5}\right) \psi \\
\psi_{L}^{C} \equiv\left(\psi_{L}\right)^{C}=\frac{1}{2}\left(1+\gamma_{5}\right) \psi^{C}=\left(\psi^{C}\right)_{R}
\end{gathered}
$$

Since fermion mass terms connect left- and right-handed fields, all of the available Dirac and Majorana mass terms are:

$$
\begin{array}{r}
\text { Dirac mass: } \mathcal{L}_{D}=m_{D}\left(\overline{\psi_{L}} \psi_{R}+\overline{\psi_{R}} \psi_{L}\right)=m_{D} \bar{\psi} \psi \\
\text { Majorana mass: } \mathcal{L}_{a}=m_{a}\left(\overline{\psi_{L}^{C}} \psi_{L}+\overline{\psi_{L}} \psi_{L}^{C}\right)=m_{a} \bar{\chi} \chi \\
\text { Majorana mass: } \mathcal{L}_{b}=m_{b}\left(\overline{\psi_{R}^{C}} \psi_{R}+\overline{\psi_{R}} \psi_{R}^{C}\right)=m_{b} \bar{\omega} \omega
\end{array}
$$

with the fields defined as:

$$
\begin{aligned}
& \psi=\psi_{L}+\psi_{R} \\
& \chi=\psi_{L}+\psi_{L}^{C}, \quad \chi^{C}=\chi \\
& \omega=\psi_{R}+\psi_{R}^{C}, \quad \omega^{C}=\omega
\end{aligned}
$$


Note how the Majorana fields are self-conjugating. This is only possible as the particle is uncharged.

The most general mass term occurs when all three of the above fields are present:

$$
\begin{aligned}
\mathcal{L}_{D M} & =m_{D} \overline{\psi_{L}} \psi_{R}+m_{a} \overline{\psi_{L}^{C}} \psi_{L}+m_{b} \overline{\psi_{R}^{C}} \psi_{R}+h . c \\
& =\frac{1}{2} m_{D}(\bar{\chi} \omega+\bar{\omega} \chi)+m_{a} \bar{\chi} \chi+\bar{\omega} \omega \\
& =\left(\begin{array}{ll}
\bar{\chi} & \bar{\omega}
\end{array}\right)\left(\begin{array}{cc}
m_{a} & \frac{1}{2} m_{D} \\
\frac{1}{2} m_{D} & m_{b}
\end{array}\right)\left(\begin{array}{l}
\chi \\
\omega
\end{array}\right)
\end{aligned}
$$

If we redefine $m_{a}$ as the mass of the left-handed neutrino and $m_{b}$ as the mass of the right-handed neutrino, the diagonalized equation gives eigenvalues of:

$$
m_{1,2}=\frac{\left(m_{L}+m_{R}\right)}{2} \pm \frac{\sqrt{\left(m_{L}-m_{R}\right)^{2}+m_{D}^{2}}}{2}
$$

\section{Seesaw Mechanism}

A special case for this method of including neutrino mass is known as the seesaw mechanism [27]. It potentially provides an explanation as to why the neutrino mass is so small compared to other fermions, so is theroretically favoured over approaches with only Dirac mass terms.

Defining $m_{L}=0$ and $m_{R} \gg m_{D}$ the mass eigenstates are:

$$
\begin{aligned}
& m_{1} \sim \frac{m_{D}^{2}}{m_{R}} \gg m_{D} \\
& m_{2} \sim m_{R}
\end{aligned}
$$

The result is one very light neutrino state with mass $m_{1}$ and a heavy state, $m_{2}$. It is the heaviness of $\omega$ (made predominantly from $\nu_{R}$ ), which makes $\chi$ (made predominantly from $\nu_{L}$ ) so light. Two interesting consequences of massive neutrinos are neutrino oscillations and the possibility of neutrinoless double beta decay.

\subsection{Neutrinoless Double Beta Decay}

Discovery of neutrinoless double beta decay would confirm the Majorana nature of neutrinos. In the process of neutrinoless double beta decay, two neutrinos are 
exchanged rather than being enitted, something that can only occur if the neutrino is its own anti-particle. Further consequences of this beyond the simple Standard Model are the violation of (total) lepton number conservation and the possibility of right-handed currents.

Double beta decay is a rare, spontaneous transition that occurs between certain nuclei with the same mass number, $A$, in which the charge, $Z$, changes by exactly two units. In this case, the more usual single beta decay is energetically less favourable, or impossible.

Double beta decay with the emission of two neutrinos $(2 \nu \beta \beta)$ was first suggested by Maria Goeppert-Mayer in 1935 [4]:

$$
(A, Z) \rightarrow(A, Z+2)+e_{1}^{-}+e_{2}^{-}+\bar{\nu}_{e_{1}}+\bar{\nu}_{e_{2}}
$$

This mode, shown in Figure $2.2 \mathrm{a}$ is allowed within the standard model without any extensions. There are five bodies in the final state and the quantity $\mathrm{E}_{e e_{2}}=\mathrm{E}_{e_{1}}+\mathrm{E}_{e_{2}}$ is continuous.

Neutrinoless Double Beta decay $(0 \nu \beta \beta)$ was first suggested by W. H. Furry in 1939 [5] after the development of the Majorana theory.

$$
(A, Z) \rightarrow(A, Z+2)+e_{1}^{-}+e_{2}^{-}
$$

It is not an allowed process within the standard model since it violates lepton number conservation and requires an $\overline{\nu_{e}}$ to exchange with a $\nu_{e}$. This process itself requires a helicity flip of the neutrino which can be achieved as a result of the massiveness of the neutrino and/or by the existence of right-handed charged lepton currents. Both of these processes are illustrated in Fig. 2.2b and 2.2c. There are three bodies in the final state resulting in a sharp peak in the $\mathrm{E}_{e e}$ spectrum at the $\mathrm{Q}$-value of the relevant transition of the given double beta decay isotope. The width of this peak is given by the detector resolution and any energy losses of the electrons.

The final mode discussed here is double beta decay with emission of a Majoron.

$$
(A, Z) \rightarrow(A, Z+2)+e_{1}^{-}+e_{2}^{-}+\chi
$$

A Majoron is a hypothetical scalar particle that could conserve the lepton number of the process if it was itself assigned a lepton number of -2 . The $\chi$ is a particle 
(a)

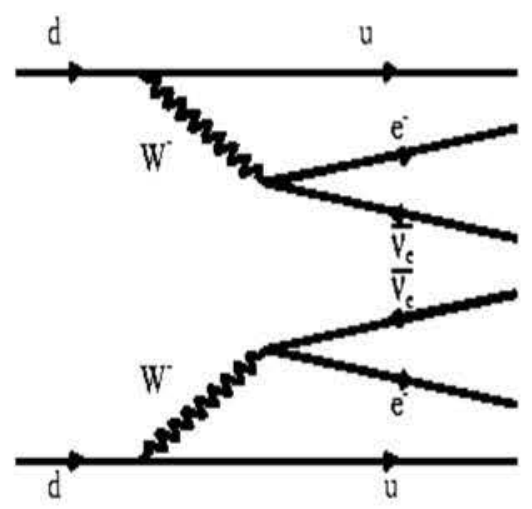

(c)

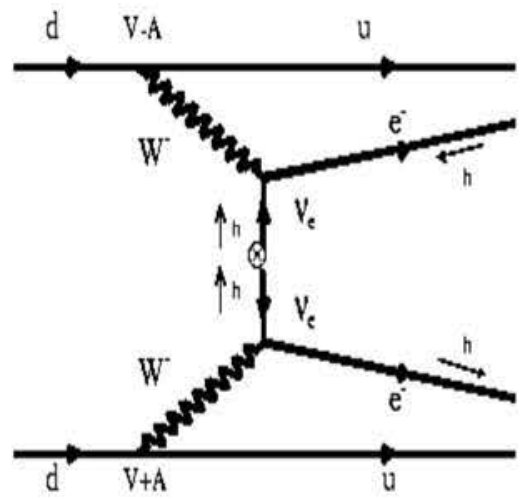

(b)

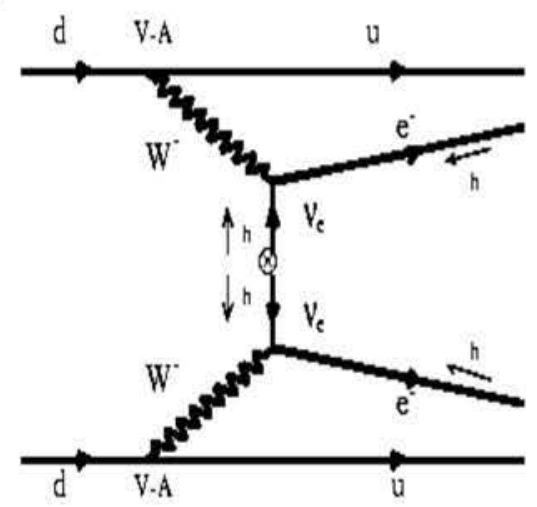

(d)

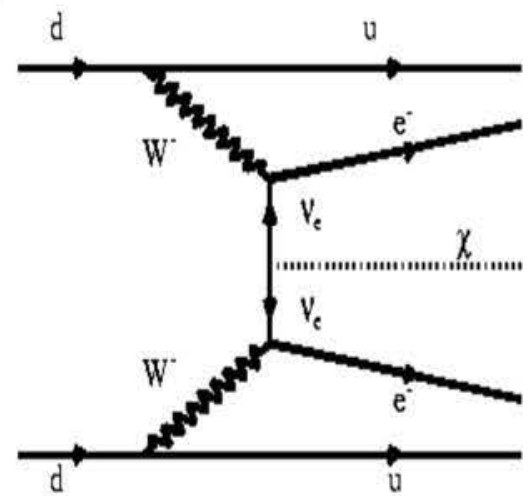

Figure 2.2: The varions modes of double beta decay and nentrimoless double beta (decay. a) Standard domble beta decay with emission of two nemtrinos. b) Standard nentrimoless domble beta decay inchuling a helicity flip resulting from massive nentrimos. c) Nentrimoless domble beta decay involving a right-hated current. d) Nentrimoless domble beta decay with emission of a Majorom.

that is momally associated with the spontameoms breaking of baryom mimus lepton mimber $(\mathrm{B}-\mathrm{L}$ ) comervation.

The characteristic: signatures from the $\mathrm{E}_{\text {eq }}$ spectra for the various motes are shown diagramatically in Figme 2.3 .

\subsection{Neutrino Oscillations}

Nentrino oscillations can provide a simple explanation for the apparent flavour changing of nemtrines that has been experimentally observed. In a simple and- 


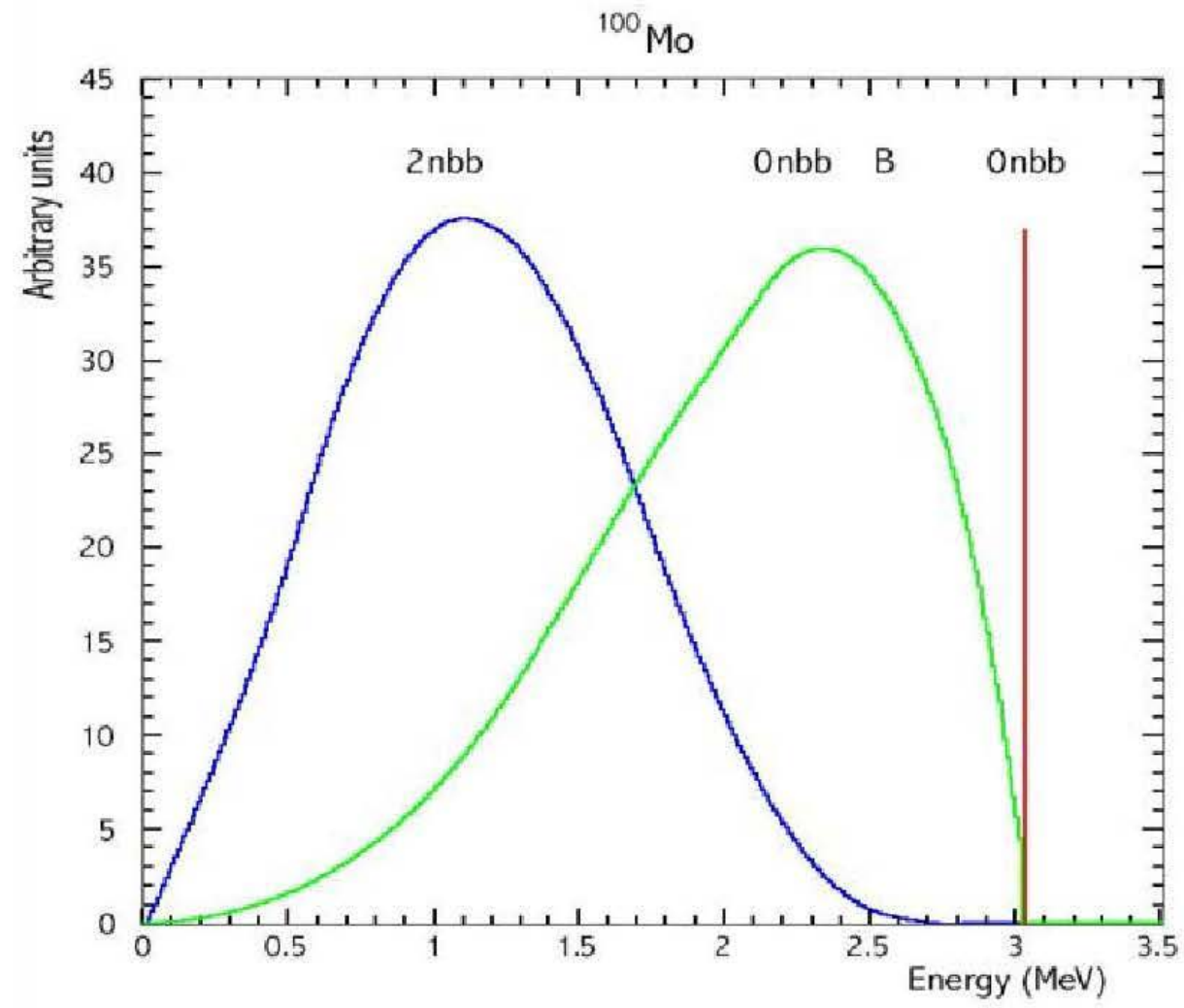

Figure 2.3: The expected observed summed-electron energy signature of two nettrino double beta decay, neutrinoless double beta decay and double beta decay with emission of a majoron.

ogy with the quark sector, massive neutrinos could have different weak and mass eigenstates related by a $3 \times 3$ unitary matrix, as shown in Equation 2.34 .

$$
\left(\begin{array}{l}
\nu_{e} \\
\nu_{n} \\
\nu_{\tau}
\end{array}\right)=\left(\begin{array}{lll}
U_{e 1} & U_{e 2} & U_{e 3} \\
U_{u 1} & U_{n 2} & U_{\mu 3} \\
U_{\tau 1} & U_{\tau 2} & U_{\tau 3}
\end{array}\right)\left(\begin{array}{c}
\nu_{1} \\
\nu_{2} \\
\nu_{3}
\end{array}\right)
$$

It is convenient to parameterize the matrix $\mathrm{U}$ in the following way [28]:

$$
U=\left(\begin{array}{ccc}
c_{12} c_{13} & s_{12 c_{13}} & s_{13} e^{-i \delta} \\
-s_{12} c_{23}-c_{12} s_{23} s_{13} e^{i \delta} & c_{12} e_{23}-s_{12} s_{23} s_{13} e^{i \delta} & s_{23} c_{13} \\
s_{12} s_{23}-c_{12 c_{23} s_{13} e^{i \delta}} & c_{12} s_{23}-s_{12 c_{23} s_{13} e^{i \delta}} & c_{23} c_{13}
\end{array}\right)
$$

where $c_{i j}=\cos \theta_{i j}, s_{i j}=\sin \theta_{i j}, \theta_{i j}$ are the neutrinos' weak mixing angles and $\delta$ is 
the CP-violating phase. The elements of this matrix can only be determined by experiment, the best values from the present data are [21]:

$$
U=\left(\begin{array}{ccc}
0.8 & 0.57 & 0 \\
0.45 & 0.5 & 0.7 \\
0.34 & 0.6 & 0.68
\end{array}\right)
$$

This shows that although all three neutrino species mix together, there may be dominant oscillation modes for each flavour. Simplifying to two flavour mixing can lead to a useful result [8]. By considering just $\nu_{e}$ and $\nu_{\mu}$, the relation:

$$
\left(\begin{array}{l}
\left|\nu_{e}\right\rangle \\
\left|\nu_{\mu}\right\rangle
\end{array}\right)=\left(\begin{array}{cc}
\cos \theta & \sin \theta \\
-\sin \theta & \cos \theta
\end{array}\right)\left(\begin{array}{l}
\left|\nu_{1}\right\rangle \\
\left|\nu_{2}\right\rangle
\end{array}\right)
$$

The initial state of a $\nu_{p}$ is given by:

$$
\left|\nu_{(x=0)}\right\rangle=\left|\nu_{p}\right\rangle=-\sin \theta\left|\nu_{1}\right\rangle+\cos \theta\left|\nu_{2}\right\rangle
$$

After some distance, $L$, the wavefunction is:

$$
\left|\nu_{(x=L)}\right\rangle=-\sin \theta e^{i p_{1} L}\left|\nu_{1}\right\rangle+\cos \theta e^{i p_{2} L}\left|\nu_{2}\right\rangle
$$

From this, the probability that the $\nu_{\mu}$ will have evolved into a $\nu_{e}$ can be derived:

$$
\begin{aligned}
P_{\nu_{k} \rightarrow \nu_{\epsilon}} & =\left|\left\langle\nu_{(x=L)} \mid \nu_{(x=0)}\right\rangle\right|^{2} \\
& =\left|-\sin \theta \cos \theta e^{i p_{1} L}+\cos \theta \sin \theta e^{i p_{2} L}\right|^{2} \\
& =\sin ^{2} 2 \theta \sin ^{2} \frac{\left(p_{2}-p_{1}\right) L}{2}
\end{aligned}
$$

where $p_{i}$ are the momenta of the species in question. Then, defining $m_{i}$ as the masses and $\mathrm{E}$ as the energy, it follows that since $\mathrm{p}_{i}^{2}=\mathrm{E}^{2}-\mathrm{m}_{i}^{2}$, in the limit $\mathrm{m}_{i}<<\mathrm{E}$, $\mathrm{p}_{i}=\mathrm{E}-\frac{m_{i}^{2}}{2 E}$ is arrived at and by defining $\Delta \mathrm{m}_{21}^{2}=\mathrm{m}_{2}^{2}-\mathrm{m}_{1}^{2}$, the relation:

$$
P_{\nu_{k} \rightarrow \nu_{\epsilon}}=\sin ^{2} 2 \theta \sin ^{2} \frac{\Delta m_{i 1}^{2} L}{4 E}
$$

is found. By manipulating the units to express energy in $\mathrm{GeV}$, length in kilometers and mass in $\mathrm{eV}$, the final transition probability is: 


$$
P_{\nu_{u} \rightarrow \nu_{\ell}}=\sin ^{2} 2 \theta \sin ^{2} \frac{1.27 \Delta m_{21}^{2} L}{E}
$$

For the baseline of the MINOS detector $(735 \mathrm{~km})$, the oscillation probability as a function of energy for values of $\Delta m_{21}^{2}$ suggested by atmospheric neutrino experiments is shown in Figure 2.4 [29].
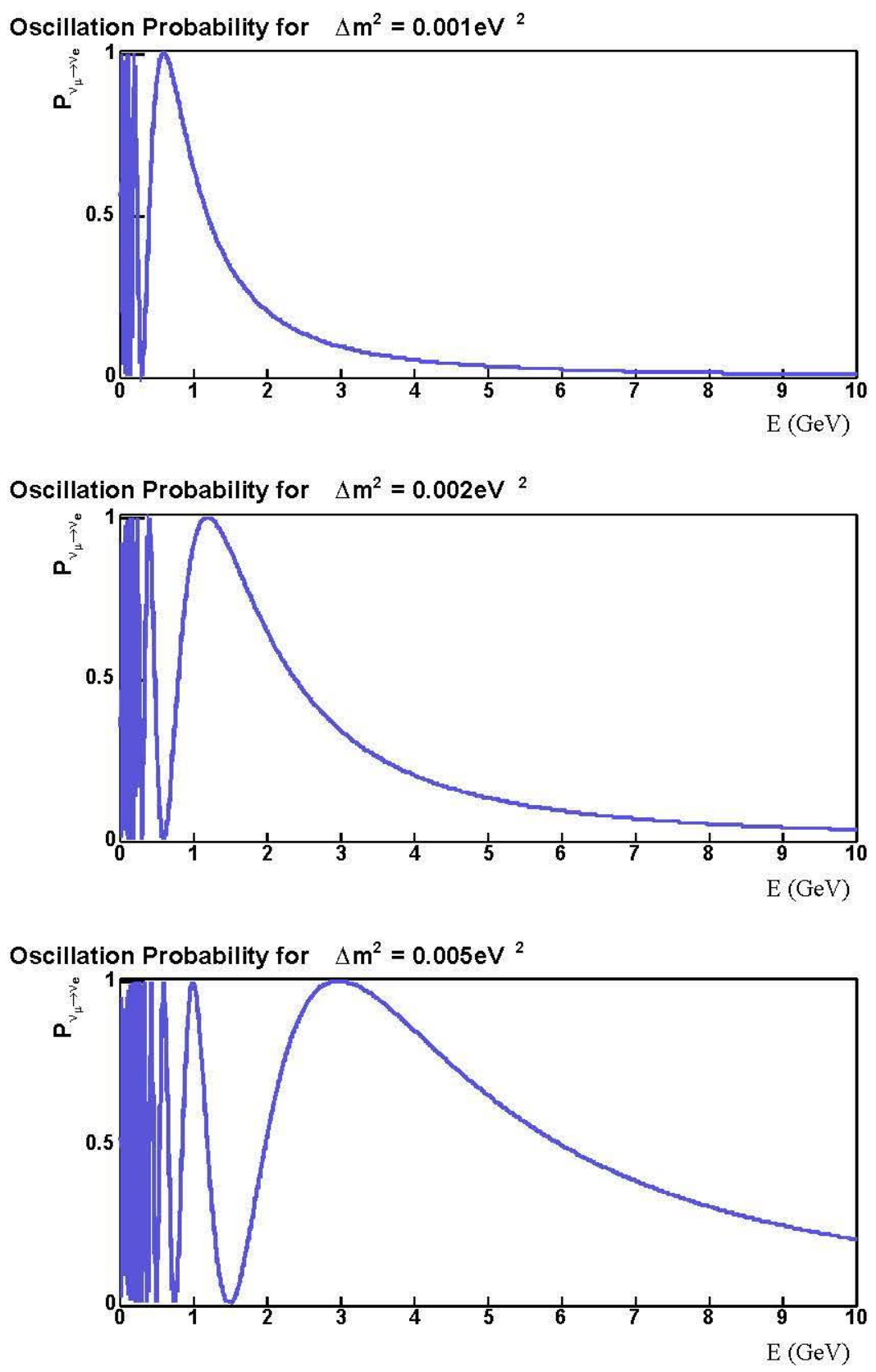

Figure 2.4: Vacuum Oscillations probability 


\subsubsection{MSW effect}

Oscillations can be enhanced when neutrinos are travelling through matter. This phenomenon is know as the Mikheyev-Smirnov-Wolfenstein (MSW) effect [30], [31]. Neutrinos have very small interaction cross-sections $\left(\sim 10^{-42}-10^{-43} \mathrm{~m}^{2}\right.$ at $\left.1 \mathrm{GeV}\right)$ but when travelling through large quantities of matter, there can be observable effects. They can undergo elastic forward scattering such that their momentum does not change. All neutrinos can interact via a neutral current channel shown in Figure 2.5. These interactions can be thought of as producing an effective refractive index, given by:

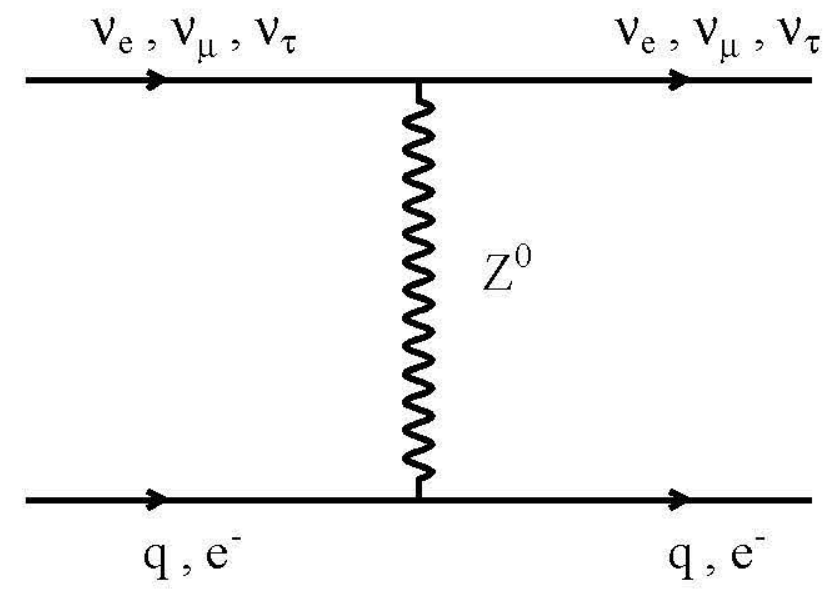

Figure 2.5: Elastic Neutral Current Forward Scattering experienced by Neutrinos. All species of neutrino can undergo this process.

$$
n=1+\sum_{\alpha} \frac{2 \pi N_{\alpha}}{p^{2}} f_{\nu \alpha}
$$

where $f_{\nu \alpha}, \alpha \in\{e, n, p\}$, is the forward scattering amplitude for the neutrino interacting with particle type $\alpha$ and $\mathrm{N}_{\alpha}$ is the number density of that particle. Neutral current interactions have no effect on oscillations since all varieties of neutrino couple equally to the $Z^{0}$. Electron (anti)neutrinos can also interact via two charged current channels shown in Figure 2.6 which contributes: 
(a)

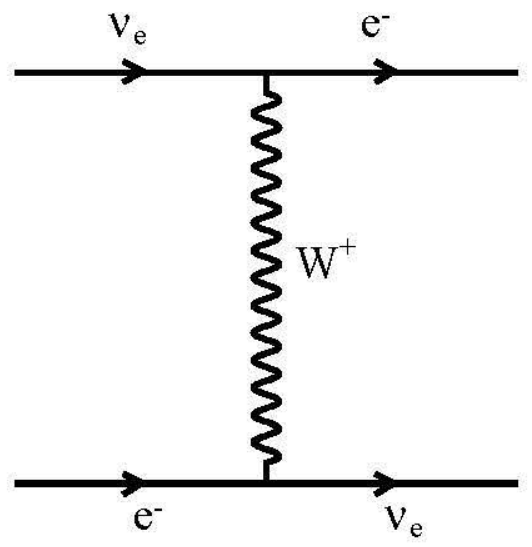

(b)

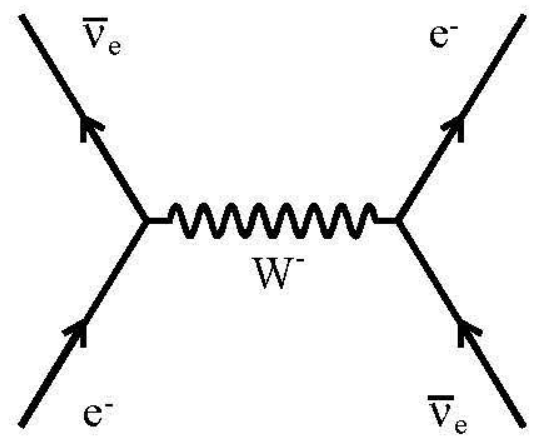

Figure 2.6: Elastic Charged Current Forward Scattering that only $\nu_{e}$ s can undergo.

$$
f_{\nu e}= \pm \frac{G_{F p}}{\sqrt{2 \pi}}
$$

where $G_{F}$ is the Fermi Constant and the plus and minus signs are for neutrinos and anti-neutrinos respectively. The refractive index introduces a phase factor of $e^{i(n-1) p x}$ leading to an additional phase of:

$$
\pm \sqrt{2} G_{F} N_{e} x
$$

This modifies the oscillation parameters $\theta$ (mixing angle) to $\theta_{m}$ and $\mathrm{l}_{\nu}$ (oscillation length) to $l_{m}$ as follows:

$$
\begin{gathered}
\sin ^{2} 2 \theta_{m}=\frac{\sin ^{2} 2 \theta}{1-2\left(l_{\nu} / l_{0}\right) \cos 2 \theta+\left(l_{\nu}^{2} / l_{0}^{2}\right)} \\
l_{m}=\frac{l_{\nu}}{\sqrt{1-2\left(l_{\nu} / l_{0}\right) \cos 2 \theta+\left(l_{\nu}^{2} / l_{0}^{2}\right)}}
\end{gathered}
$$

where $l_{\nu}=4 \pi \mathrm{E} / \delta \mathrm{m}_{21}^{2}$ is the vacuum oscillation length, $\rho$ is the density of the medium being traversed and $\mathrm{l}_{0}=4 \pi \mathrm{a} / \rho$.

The quantity, a, is defined as:

$$
a=\frac{1}{2 \sqrt{2} G_{F} N_{e}}
$$


This leads to two interesting scenarios. Firstly is the case where a beam of monoenergetic neutrinos passes through a medium of varying density. A significant enhancement of oscillations occurs in the layer of density:

$$
\rho_{r e s}= \pm a \frac{\Delta m_{21}^{2} \cos 2 \theta}{E}
$$

This is thought to account for the majority of neutrino oscillations that occur in the sun. When a beam of neutrinos of continuous energy passes through a medium of constant density, there is an oscillation resonance in the portion of the spectrum where:

$$
E_{r e s}= \pm a \frac{\Delta m_{21}^{2} \cos 2 \theta}{\rho}
$$

This is the principle of long baseline neutrino oscillation experiments since a resonance can occur for even very small values of the vacuum mixing angle. In addition, the mass hierarchy of neutrinos can be probed in this way since resonant enhancement of neutrinos is only possible if $m_{2}>m_{1}$ and of antineutrinos if $m_{2}<m_{1}$. Figure 2.7 shows the enhancement of the mixing angle for different values of the matter density. The effective $\Delta m_{21}^{2}$ is shown for the same densities in Figure 2.8. The effect on these on the oscillation probability is shown in Figure 2.9. The effects of $\sin ^{2} 2 \theta_{\text {matter }}$ and $\Delta \mathrm{m}_{\text {matter }}^{2}$ work against each other, resulting in oscillation probabilities in the earth being similar to those in a vacuum. In each figure, three different values of the vacuum mixing angle are shown. 

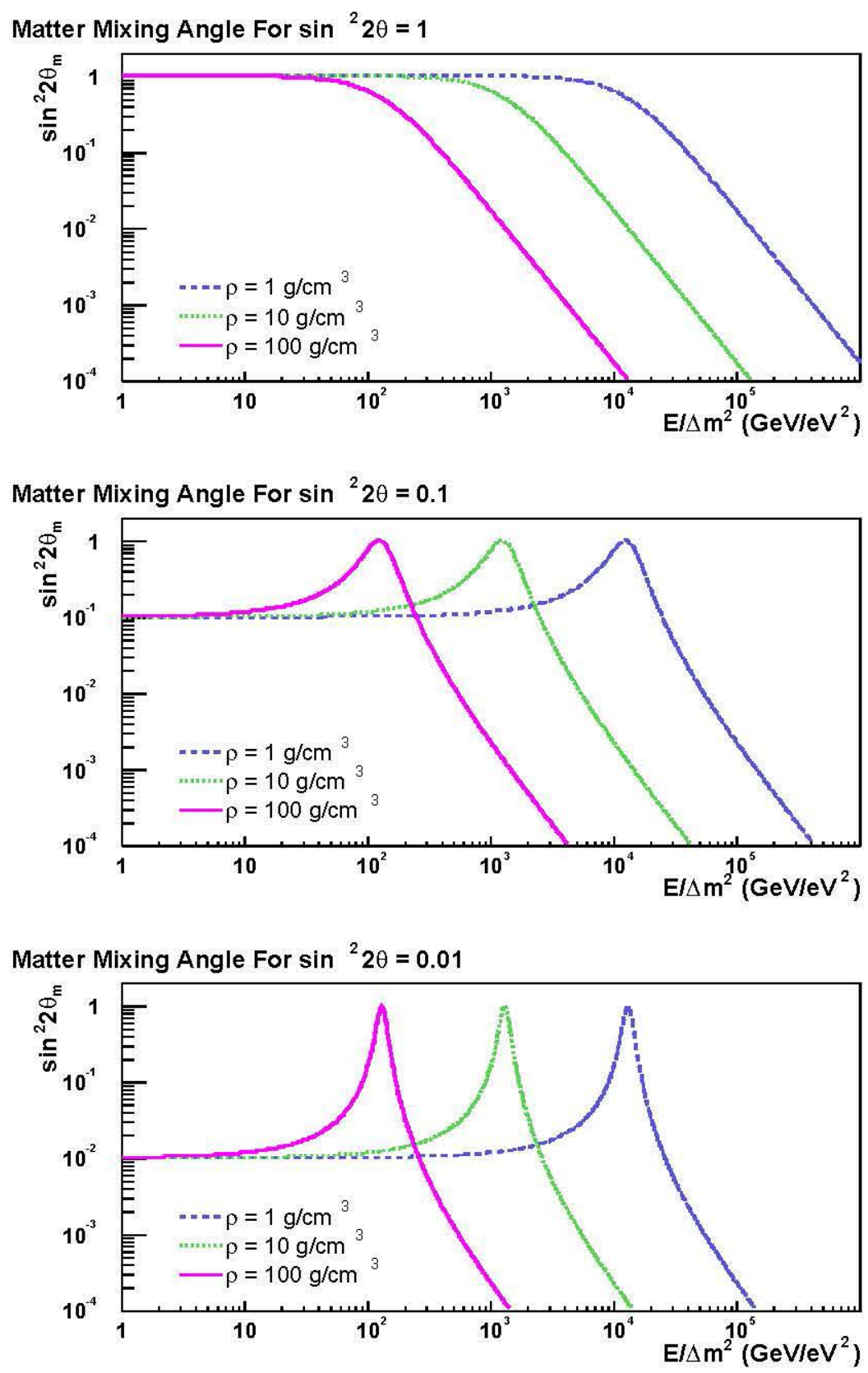

Figure 2.7: The neutrino mixing angle is modified for neutrinos travelling through matter. This plot shows the matter mixing angle for various neutrino energies at various matter densities. 

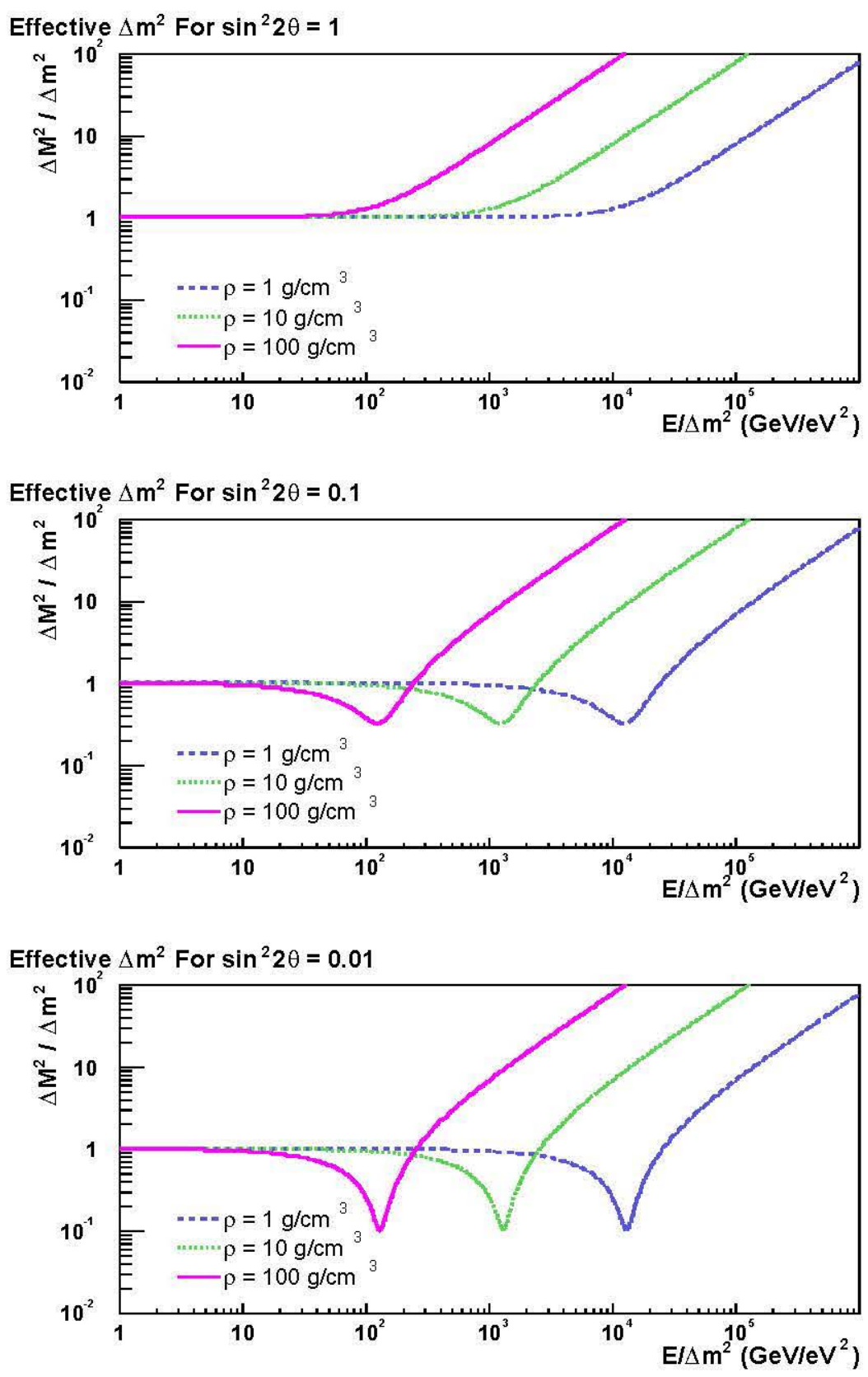

Figure 2.8: $\Delta \mathrm{m}^{2}$ is also modified for neutrinos travelling through matter. This plot shows the ratio of the modified $\Delta \mathrm{m}^{2}$ to $\Delta \mathrm{m}_{\text {vacuum }}^{2}$ for various neutrino energies at various matter densities. 

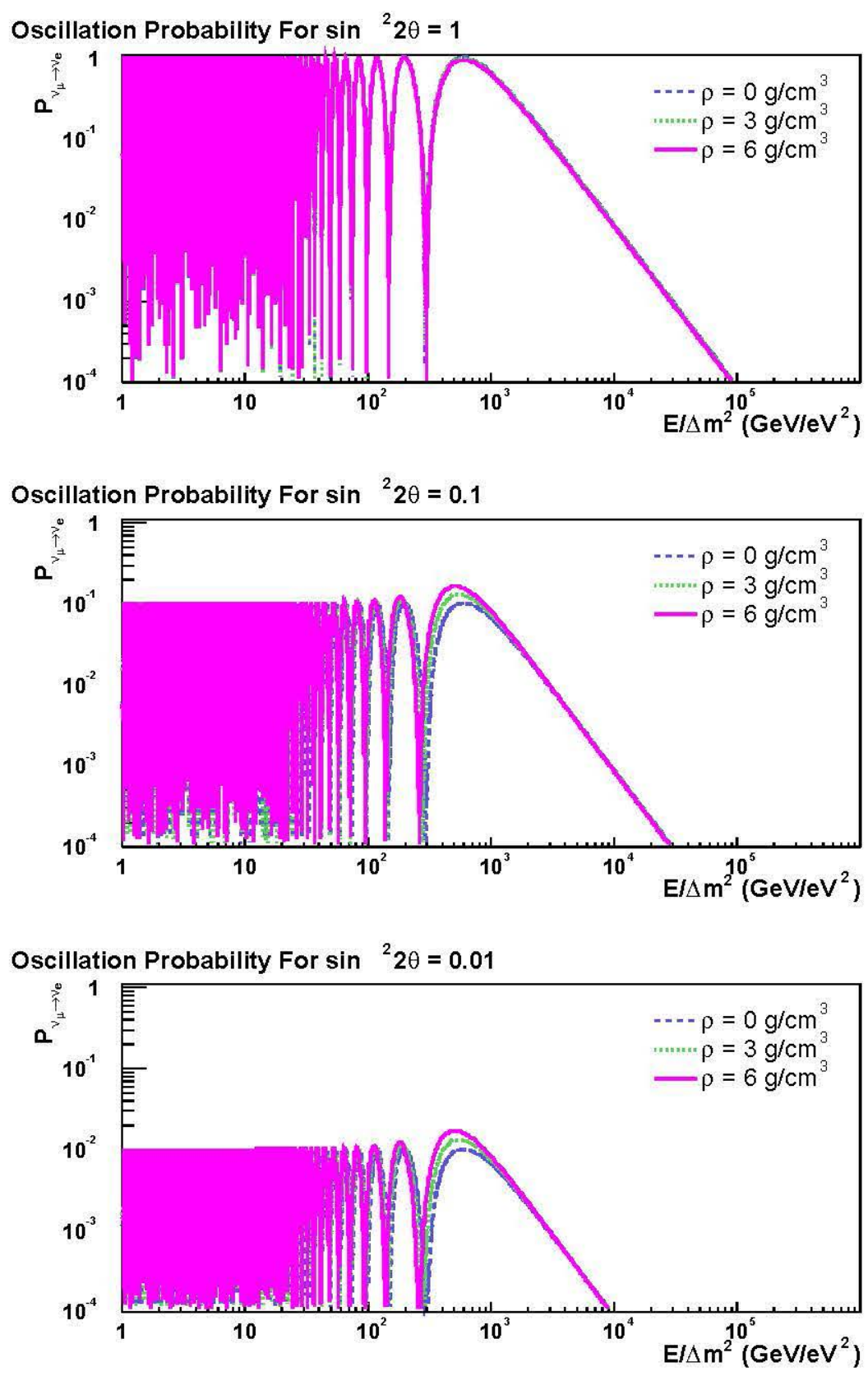

Figure 2.9: The effects of $\sin ^{2} 2 \theta_{\text {matter }}$ and $\Delta \mathrm{m}_{\text {matter }}^{2}$ work against each other, resulting in oscillation probabilities in the earth being similar to those in a vacuum. 


\section{Chapter 3}

\section{The History of Neutrino Experiments}

\section{$3.1 \quad$ Introduction}

The concept of the neutrino was suggested by Pauli in 1930 to explain the apparent non-conservation of energy seen in beta decay experiments in the late 1920 s and early $1930 \mathrm{~s}$ [1]. Unlike those seen in alpha and gamma ray experiments, the spectra from beta decay were continuous and more like that expected from a two-body final state. In 1931 Fermi formally developed his theory of beta decay within the framework of Quantum Electrodynamics [1,3] and named the elusive particle that was a component of the theory, the neutrino meaning little neutral one. Fermi's theory was very successful but direct detection of a neutrino seemed impossible owing to its incredibly weak interaction strength. But in June 1956 at the Savannah River reactor, Reines and Cowen observed $3.0 \pm 0.2$ events per hour above all backgrounds in their water and scintillator $\left(\mathrm{CdCl}_{2}\right)$ detector $[6]$. This was attributed to the inverse beta decay interaction: $\bar{\nu}_{e}+p \rightarrow n+e^{+}$.

Three neutrino species are now known to exist: the electron neutrino $\nu_{e}$, the muon neutrino $\nu_{\mu}$, first seen directly by Schwartz and collaborators in Brookhaven in 1961 and the tau neutrino, $\nu_{\tau}$, observed in the DONUT experiment in 2000. This completed our picture of leptons in nature: three charged $\left(\mathrm{e}^{-}, \mu^{-}\right.$and $\left.\tau^{-}\right)$with three neutral partners $\left(\nu_{e}, \nu_{p}\right.$ and $\left.\nu_{\tau}\right)$ and six corresponding antileptons. Measurement of 
the width of the $Z^{0}$ resonance at CERN $[13,14,15,16]$ showed that it couples to three light, active neutrinos only.

But the story was far from complete. The experiments of $\mathrm{Wu}$ et al. [7] and Goldhaber et al. [32] showed that the weak interaction has a strange property in that it maximally violates parity, resulting in the fact that all neutrinos are created in a left-handed state. The apparent non-existence of right-handed neutrinos implied that they travelled at the speed of light and were massless. As a result, right handed neutrinos were not included in the Standard Model of particle physics.

The first indication that neutrinos exhibited behaviour not predicted by the Standard Model came from observations of solar neutrinos in 1968 by Ray Davis at the Homestake Mine [10]. Fewer $\nu_{e}$ were observed than expected but later experiments confirmed that the total number of neutrinos arriving at the earth was correct. Since then, mounting evidence suggests that the neutrino's weak eigenstates are mixtures of their mass eigenstates and that they can 'oscillate' from one flavour to another as they travel. This is only possible if neutrinos have mass, although it still may be very small. Since then a number of experiments have been developed to try and measure the mass of the neutrino and to determine its exact nature and the mechanism by which it is able to change flavour.

\subsection{Measurements of Neutrino Masses}

There are two classes of experiment that address the question of neutrino mass: Direct and Indirect. Direct techniques make few a priori assumptions about the neutrino's properties since the measurements tend to be based on kinematic observables. Indirect measurements can have requirements such as lepton number violation and may not probe the absolute mass of the neutrino. Direct measurements probe the quantity:

$$
\overline{M_{\nu_{\alpha}}}=\sqrt{\sum_{i=1}^{k}\left|U_{\alpha i}\right|^{2} m_{\nu_{i}}^{2}}
$$

which is the weighted average mass of a particular neutrino species. Here, $\alpha=(\mathrm{e}, \mu$, $\tau), \mathrm{U}_{\alpha i}$ are the amplitudes in the lepton mixing matrix and the sum over $\mathrm{k}$ includes 
all mass eigenstates that are kinematically allowed for a particular measurement.

\subsection{Direct Techniques}

Studies of the end-point of the electron energy spectrum from tritium decay have been used to search for non-zero electron neutrino masses via the process:

$$
{ }_{1}^{3} \mathrm{H} \rightarrow{ }_{2}^{3} \mathrm{He}+\mathrm{e}^{-}+\bar{\nu}_{e}
$$

If the electron neutrino does have a mass, potentially measurable distortions will occur to the end-point of the resultant electron energy spectrum (See Figure 3.1). The measurement is complicated by the fact that very few decays occur in the region of interest and corrections must be made for nuclear screening effects and final state interactions of the tritium itself. The best result currently comes from the University of Mainz which sets an upper limit on $\overline{M_{\nu_{\epsilon}}}$ of $2.2 \mathrm{eV}$ at $95 \%$ C.L. In the future an international project to build a next generation $\beta$-spectrometer, $\mathrm{KATRIN}^{1}$, is expected to bring the upper limit down to $\sim 0.3 \mathrm{eV}$ [33]. An alternative method uses a cyrogenic calorimeter to detect decays of ${ }^{187}$ Re. This has the advantage of a very low transition energy and therefore better statistics in the region of interest. [34].

An upper limit on the $\nu_{p}$ mass can be obtained by studying the decay:

$$
\pi^{+}(\text {at rest }) \rightarrow \mu^{+}+\nu_{\mu}
$$

This process is a two-body decay, so the masses of the muon and pion and the muons momentum are all that is required to set a limit on the neutrinos mass:

$$
{\overline{M_{\nu}}}^{2}=m_{\pi}^{2}+m_{\mu}^{2}-2 m_{\pi} \sqrt{p_{\mu}^{2}+m_{\mu}^{2}}
$$

The latest results from the Paul Scherrer Institute in Switzerland [35] give a value of $170 \mathrm{keV} / \mathrm{c}^{2}$ at $90 \%$ C.L.

Limits on the mass of the $\nu_{\tau}$ are obtained by studying the decays:

\footnotetext{
${ }^{1}$ KArlswuhe TRItium Neutrino experiment,
} 


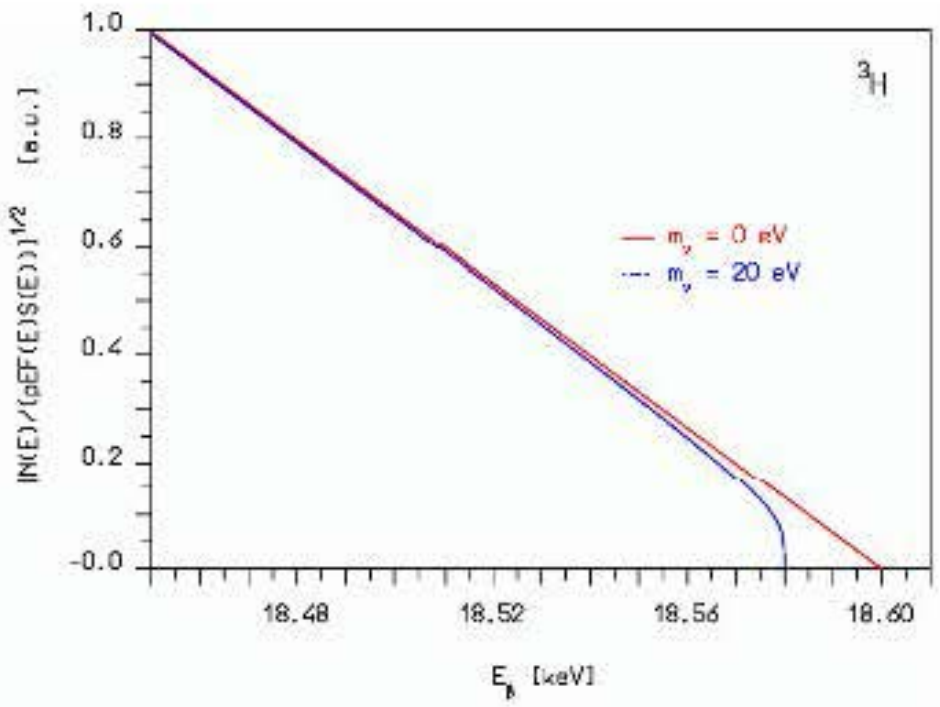

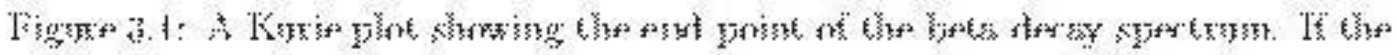

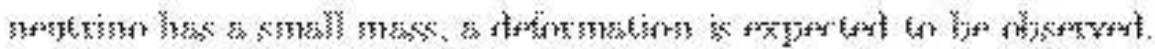

$$
r \rightarrow i k+i k+i
$$

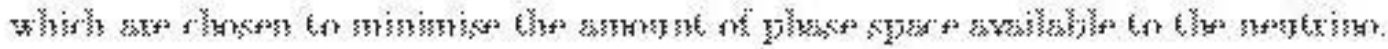

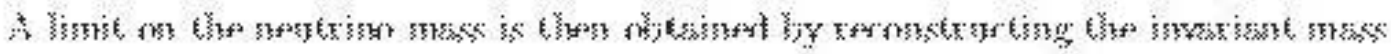
of 6 Q

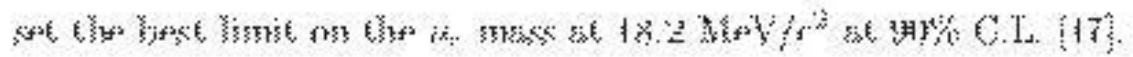

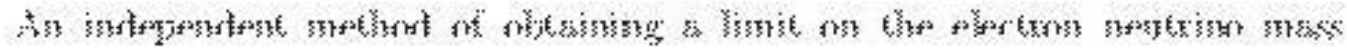

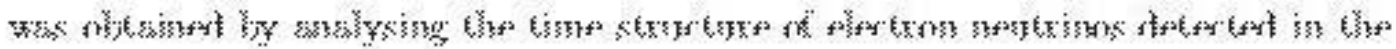

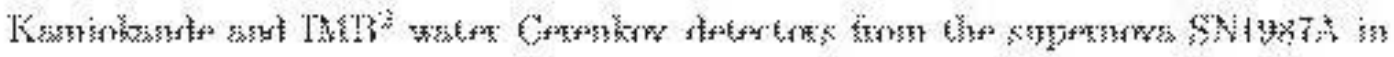

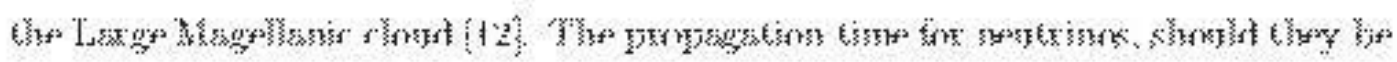

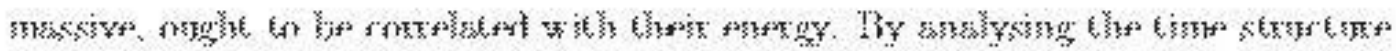
กी Th:

औसs

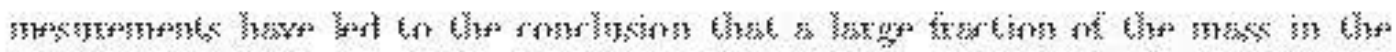

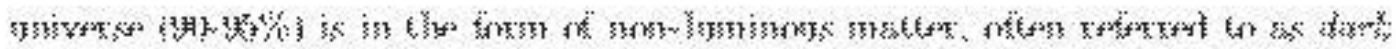

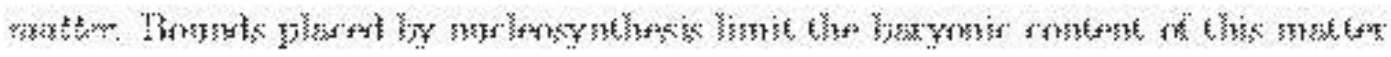

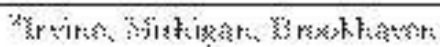


to $10 \%$. However, neutrinos are the second most abundant particle in the universe $\left(n_{\nu}=1 / 3 n_{p h o t o n s}\right)$ and so even if they have a very small mass, they could still contribute towards a significant fraction of the dark matter. In order to prevent the universe being closed (i.e. $\Omega>1$, not observed) the sum of all neutrino masses must satisfy the relation:

$$
\sum_{e, \nu, \tau} m_{\nu}<91.5 \Omega_{\nu} h^{2} \mathrm{eV}
$$

where $h$ is the Hubble Constant. This implies:

$$
\sum_{e, \nu, \tau} m_{\nu}<0.70 \mathrm{eV}[37]
$$

\subsection{Indirect Techniques}

Although many indirect techniques for measuring the mass of the neutrino can be very precise, they are constrained to measuring a quantity that is some function of the neutrino mass and not the mass itself. Two major branches of experimental physics today focus on two particular measurements: the effective Majorana mass and the mass difference squared between the species of neutrino.

\subsubsection{The Effective Majorana Mass of the Neutrino}

The effective Majorana mass of the neutrino is defined as:

$$
<m_{i}>_{\alpha}=\sum_{i} U_{\alpha i}^{2} m_{i}
$$

where the sum over $\mathrm{i}$ covers the mass eigenstates and $\alpha$ are the weak eigenstates, $\mathrm{e}$ $\mu$ and $\tau$. Determination of the effective Majorana mass is reliant on the fact that the neutrino is a Majorana particle and not a Dirac one. The most promising test of the neutrino's Majorana verses Dirac nature is neutrinoless double beta decay $(0 \nu \beta \beta$, see Figure 3.2 a). This type of interaction can be thought of as a double weak decay with an exchange of virtual neutrinos.

Before considering $0 \nu \beta \beta$ as a window on the neutrino mass, it must first be established that the simpler mechanism, double beta decay with emission of two 
neutrinos $(2 \nu \beta \beta$, Figure $3.2 \mathrm{~b})$, does exist in nature. It was first shown that it indeed does by T. Kirsten and his co-workers in the late 1960's [38]. A geochemical method was used, based on the search for daughter products that have accumulated in ancient minerals over billions of years. A $17 \mathrm{~g}$ sample of natural tellurium ore $\left({ }^{130} \mathrm{Te}\right.$ ) was studied by mass spectroscopy and chemical analysis to seach for an excess of ${ }^{130} \mathrm{Xe}$. The half-life for this decay was found to be $T_{1 / 2}^{\beta 3}\left({ }^{130} \mathrm{Te}\right)=2.19$ x $10^{21}$ years [39]. Soon afterwards, experiments studying ${ }^{128} \mathrm{Te}[40,41]$ and ${ }^{82} \mathrm{Se}$ $[43,42]$ were performed. A similar procedure, known as a radiochemical method, can be used to study ${ }^{238} \mathrm{U},{ }^{232} \mathrm{Th}$ and ${ }^{244} \mathrm{Pu}$. In these cases, the energy of alpha particles emitted by the daughter nuclei are measured.

(a)

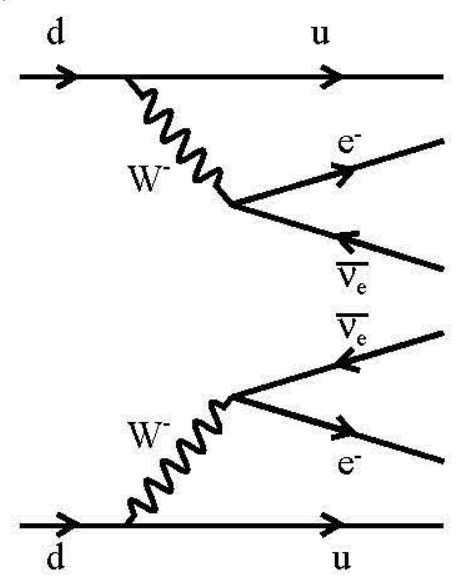

(b)

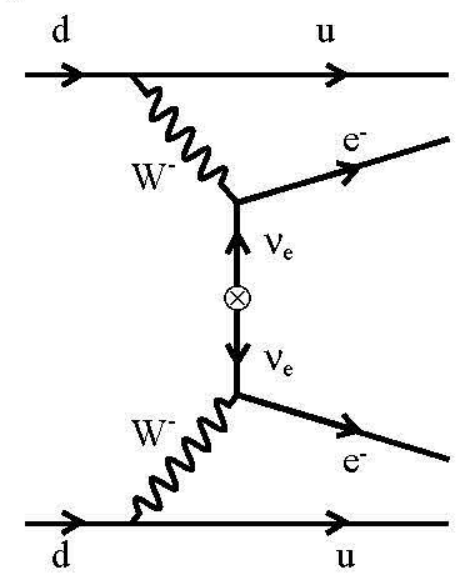

Figure 3.2: Feynman diagrams illustrating two forms of double beta decay. On the left is a diagram showing conventional double beta decay where two neutrinos are produced. On the right is neutrinoless double beta decay which can be thought of as a virtual exchange of neutrinos in the final state.

The exact mode of the $\beta \beta$ decay cannot be probed by any of these experiments since all the information about the electron energies is lost. In order to distinguish between decay modes, extremely efficient background suppression is required, or additional information such as that from particle tracking. These direct techniques for double beta decay measurement tend to fall into two categories: Calorimetric and Tracking. 


\section{Calorimetric}

Germanium based detector experiments have a very good detection efficiency and excellent energy resolution making them very suitable for $0 \nu \beta \beta$ decay searches.

The Heidelberg-Moscow experiment $[44,45,46]$ employs ${ }^{76} \mathrm{Ge}$ as both the double beta emitter and the active detector component. Five high purity germanium detectors (HPGe) were installed in the Gran Sasso underground laboratory, and comprised $10.96 \mathrm{~kg}$ of germanium, enriched such that $86 \%$ of the mass is the isotope ${ }^{76} \mathrm{Ge}$. The Heidelberg-Moscow experiment claims to have detected evidence of neutrinoless double beta decay. After a recent re-evaluation of their data, they have published at the $4.2 \sigma$ level $(99.9973 \% \mathrm{c} .1)$ the value of:

$$
\left\langle m_{\nu}\right\rangle=0.1-0.9 \mathrm{eV}[47]
$$

taking a $50 \%$ error in the nuclear matrix element into account. The best fit value is $0.4 \mathrm{eV}$.

Another experiment currently ruming, $\operatorname{IGEX}^{3}[48,49]$, has $8 \mathrm{~kg}$ of similarly enriched ${ }^{76} \mathrm{Ge}$ detectors at the Baksan and Canfranc underground laboratories. In the future, the GENIUS ${ }^{4}$ and Majorana experiments hope to use similar techniques with much larger quantities of germanium.

Cryogenic (bolometer) detectors can measure beta decay based on the fact that the heat capacity at low temperatures of a diamagnetic and dielectric crystal (such as $\mathrm{TeO}_{2}$ ) is proportional to the cube of the ratio between the operating and Debye temperatures. Therefore, in a low temperature enviromment, a tiny energy release by a particle can be detected by the increase in temperature of the absorber. Two experiments using this technique to investigate $0 \nu \beta \beta$ decay in natural $\mathrm{TeO}_{2}$ are MI-DBD 5 and CUORICINO [50] in Gran Sasso. In around a year's data taking; CUORICINO currently sets a lower limit of $\mathrm{T}_{1 / 2}^{0 \nu \beta 3} \geq 1.0 \times 10^{24}$ years at $90 \%$ C.L. which corresponds to:

$$
<m_{\nu}>\leq 0.26-1.4 \mathrm{eV}[51]
$$

\footnotetext{
${ }^{3}$ International Germanium EXperiment,

${ }^{4}$ GErmanium in liquid NItrogen Underground Setup

"MIrro Double Beta Decay
} 
After 3 years, CUORICINO intents to upgrade the experiment from an active mass of $42 \mathrm{~kg}$ of $\mathrm{TeO}_{2}$ to $775 \mathrm{~kg}$, a proposal known as CUORE ${ }^{6}$.

\section{Particle Track Reconstruction}

A completely different class of $0 \nu \beta \beta$ decay experiments utilise passive sources, inside a detector which is capable of measuring the energy of emitted particles and providing track reconstruction. This method has the advantages of providing very good background reduction and allows a large variety of isotopes to be studied. Early attempts used cloud chambers or time projection chambers (TPC) with sources in the form of thin foils (to limit energy loss in the material) or as the TPC gas itself (in the case of ${ }^{136} \mathrm{Xe}$ ). The proposed experiment, EXO, ${ }^{7}$ builds on this design, using a TPC with background suppression based on laser tagging ${ }^{136} \mathrm{Ba}^{++}$ions produced by $\beta \beta$ decay. A sensitivity to $0.01 \mathrm{eV}$ is expected.

The design of the NEMO detectors is a wire chamber which provides three dimensional tracking and is combined with a calorimeter to measure the energy of electrons, positrons and photons. The first generation of the experiment focussed exclusively on the $\beta \beta$ decay of ${ }^{100}$ Mo whereas NEMO-2 also measured $2 \nu \beta \beta$ decays in ${ }^{82} \mathrm{Se},{ }^{116} \mathrm{Cd}$, and ${ }^{96} \mathrm{Zr}$. The most recent version, NEMO-3, contains larger quantities of the isotopes ${ }^{100} \mathrm{Mo},{ }^{82} \mathrm{Se},{ }^{116} \mathrm{Cd},{ }^{130} \mathrm{Te},{ }^{150} \mathrm{Nd},{ }^{96} \mathrm{Zr}$, and ${ }^{48} \mathrm{Ca}$. The NEMO-3 experiment is fully operational, taking data now and is described in Chapter 6 . No evidence for $0 \nu \beta \beta$ has so far been seen with $\sim 7 \mathrm{~kg}$ of ${ }^{100} \mathrm{Mo}$ and $\sim 1 \mathrm{~kg}$ of ${ }^{82} \mathrm{Se}$. The corresponding limits are $\mathrm{T}_{1 / 2}(0 \nu \beta \beta) \geq 4.6 \times 10^{23}$ years for ${ }^{100} \mathrm{Mo}$ and $\mathrm{T}_{1 / 2}(0 \nu \beta \beta)$ $\geq 1.0 \times 10^{23}$ years for ${ }^{82} \mathrm{Mo}(90 \%$ C.L.). With uncertainties in the nuclear matrix element calculations included, the limits on the effective Majorana neutrino mass are:

$$
\begin{aligned}
& { }^{100} \mathrm{Mo}<0.7-2.8 \mathrm{eV} \\
& { }^{82} \mathrm{Se}<1.7-4.9 \mathrm{eV}
\end{aligned}
$$

The Japanese experiment ELEGANTS $V^{3}$, situated in the Oto Cosmo Observa-

\footnotetext{
${ }^{6}$ Cryogenic Underground Observatory for Rare Events

${ }^{7}$ Enriched Xenon $\beta \beta$ decay Observatory
} 
tory utilises a $\beta-\gamma$ spectrometer which consists of three drift chambers for particle tracking, and a calorimeter. Two high purity ${ }^{100} \mathrm{Mo}$ sources are used, with a total mass of $171 \mathrm{~g}$.

Two recently proposed projects are MOON ${ }^{8}$ and Super NEMO. MOON aims to study both $\beta \beta$ decay and solar neutrinos using 40 tons of natural molybdenum foils (equivalent to 3.3 tons ${ }^{100} \mathrm{Mo}$ ) interleaved with plastic scintillator modules. The expected sensitivity is $\sim 0.03 \mathrm{eV}$. Super NEMO hopes to employ $100 \mathrm{~kg}$ of ${ }^{82} \mathrm{Se}$ foils sandwiched between scintillator walls. This relatively low budget experiment can reach sensitivity of $0.05-0.11 \mathrm{eV}$.

\subsubsection{The Mass Difference Squared and mixing between neutrino species}

The discovery of a solar $\nu_{e}$ and atmospheric $\nu_{k}$ deficit has created a huge branch of experimental neutrino physics: neutrino oscillations; the seemingly most likely solution to these two problems. Neutrino oscillation measurements offer the most sensitive method of probing neutrino mass but they can only probe the mass difference squared $\left(\Delta \mathrm{m}_{i j}^{2}=\mathrm{m}_{i}^{2}-\mathrm{m}_{j}^{2}\right)$ between the neutrino species. Although this gives information on the neutrino mass scale it does not advance our knowledge of the absolute masses $\mathrm{m}_{1}, \mathrm{~m}_{2}$ and $\mathrm{m}_{3}$.

Experiments intended to measure the $\Delta \mathrm{m}^{2} \mathrm{~s}$ and the closely related quantities $\theta_{12}, \theta_{23}$ and $\theta_{13}$ (the neutrino mixing angles) fall into the several categories depending on the source of the neutrinos: Solar, Atmospheric, Reactor and Accelerator.

\section{Solar Neutrinos}

Neutrinos are generated in the core of the Sun in nuclear fusion reactions. Two cycles of processes occur: the proton-proton (pp) chain and the Carbon-NitrogenOxygen cycle. These produce neutrinos with a spectrum of energies, some discrete and some continous, averaging to around $1 \mathrm{MeV}$. Neutrinos from the Sun were first detected via the reaction:

\footnotetext{
${ }^{8}$ MOlybdemm Observatory Of Neutrinos
} 


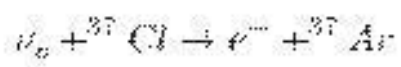

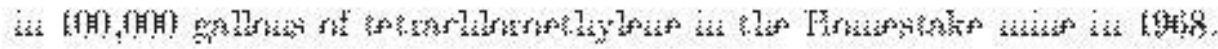

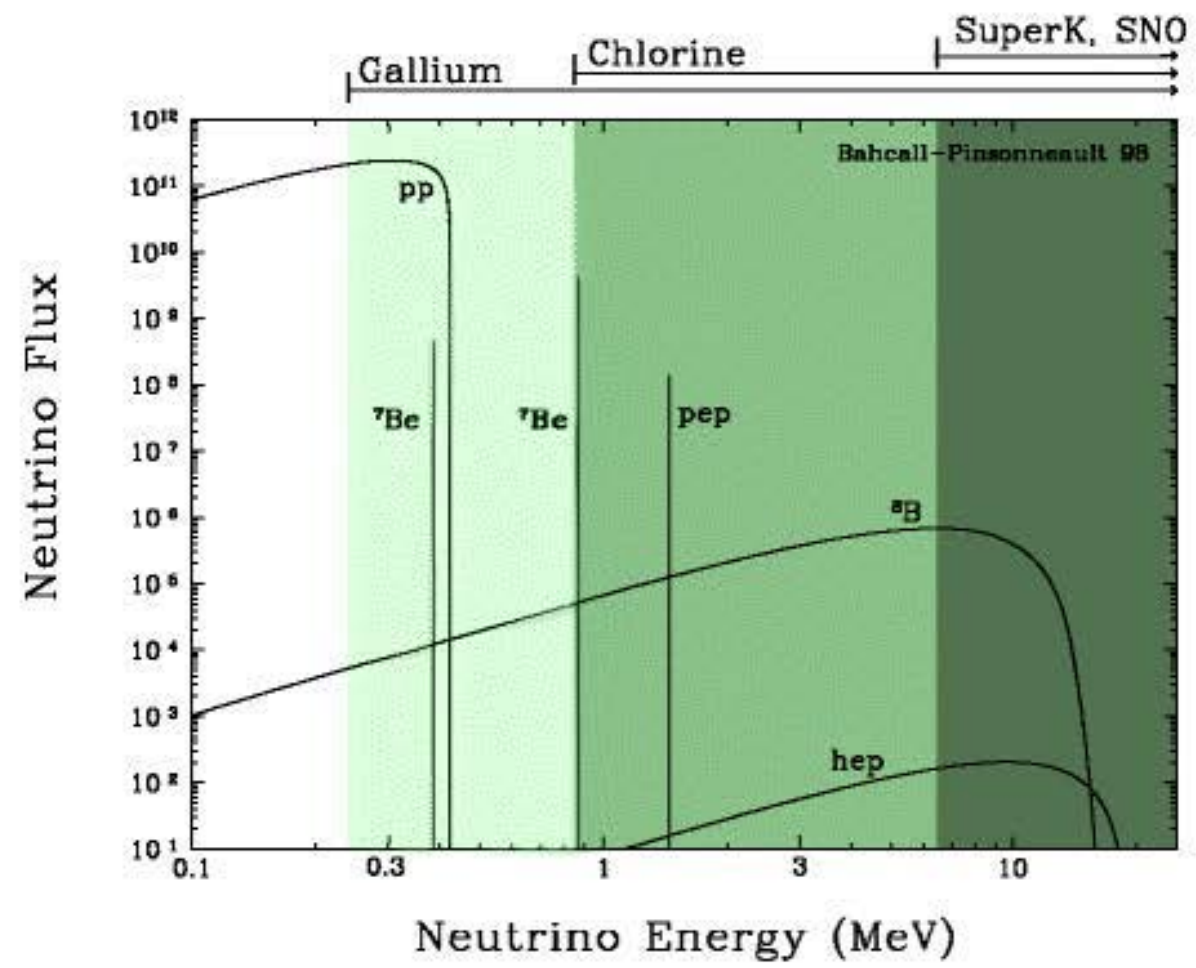

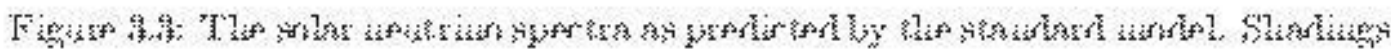

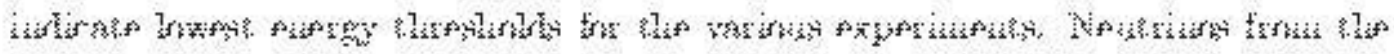

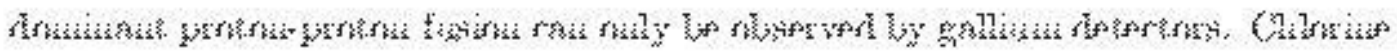

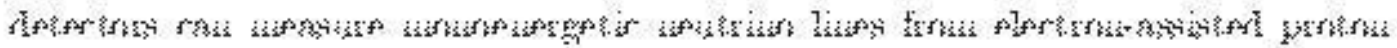

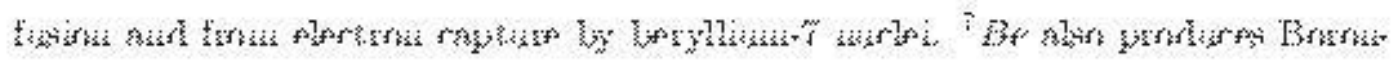

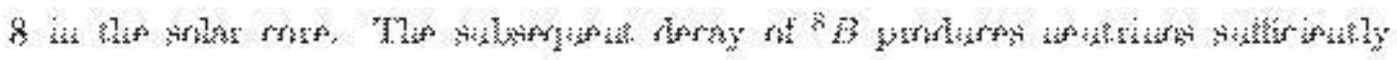

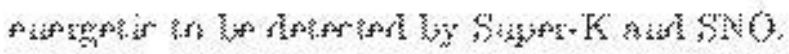

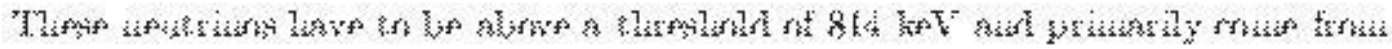

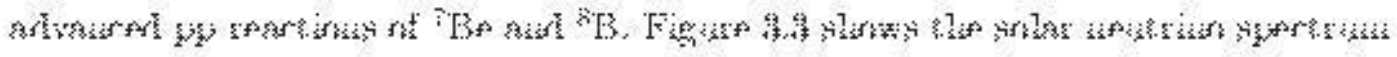

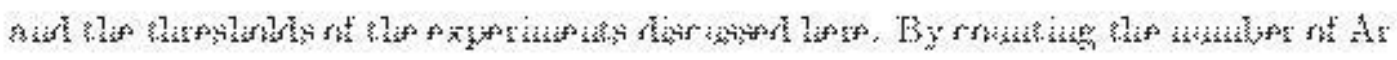

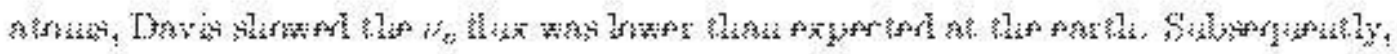


$\mathrm{SAGE}^{9}[52,53], \mathrm{GALLEX}^{10}[54,55]$ and $\mathrm{GNO}^{11}[56]$ have confirmed the result by utilising gallium in the reaction:

$$
\nu_{e}+{ }^{71} G a \rightarrow e^{-}+{ }^{71} G e
$$

This reaction occurs at a lower energy threshold (233 keV) and is sensitive to a region of the solar neutrino spectrum where the expected flux is much larger.

Kamiokande [11] and Super-Kamiokande (Super-K) [67, 68] were able to verify the result independently by using a different detection technique. Located $1000 \mathrm{~m}$ underground in the Kamioka mine in Japan, these detectors relied on the fact that neutrinos will elastically scatter off electrons in water, producing rings of Cerenkov light. Kamiokande contained $3 \mathrm{kT}$ of water and Super-K contains $50 \mathrm{kT}$ and they both utilised large arrays of photomultiplier tubes to measure the energy of the recoil electrons and also determine the original neutrinos direction. These experiments were able to show that the measured neutrinos were indeed coming from the sun.

The SNO ${ }^{12}$ experiment is also a water Cerenkov detector, but it uses heavy water $\left(\mathrm{D}_{2} \mathrm{O}\right)$ which allows it to distinguish between $\nu_{e}$ charged current (CC) events and all neutrino neutral current events (NC).

In regular water they are indistinguishable (See Figure. 3.4) but in $\mathrm{D}_{2} \mathrm{O}$ the following interactions are available:

$$
\begin{aligned}
& C C: \quad \nu_{e}+{ }^{2} H \rightarrow p+p+e^{-} \\
& N C: \quad \nu+{ }^{2} H \rightarrow p+n+\nu(\text { above } 2.2 \mathrm{MeV})
\end{aligned}
$$

since the proton and neutron that constitute the deuteron are only loosely bound and the cross-section for their interaction with a neutrino becomes much larger than that of an electron. Therefore, provided the energy transferred is above the deuteron's binding energy of $2.2 \mathrm{MeV}$, a neutron can be liberated which can be detected by characteristic photons when it is subsequently captured. This is distinct from the Cerenkov signal that is produced by the electron in the case of a CC interaction.

\footnotetext{
${ }^{9}$ Russian-American Gallium Solar Neutrino Experiment

${ }^{10}$ GALLium European eXperiment

${ }^{11}$ Gallium Neutrino Observatory

${ }^{12}$ Sudbury Neutrino Observatory
} 
(a)

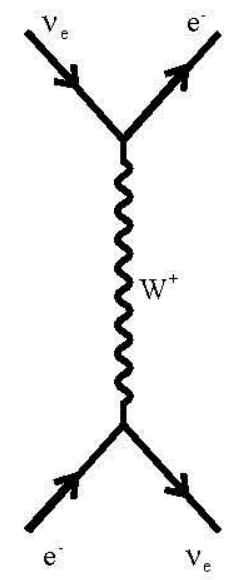

(b)

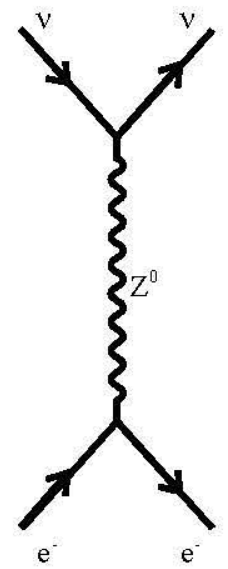

(c)

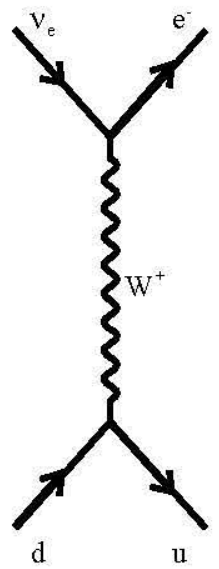

(d)

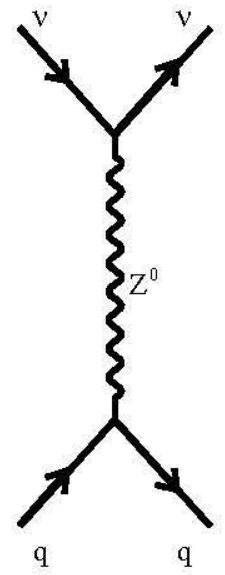

Figure 3.4: On the left, (a) shows a normal charged current interaction that occurs between an electron neutrino and an electron in normal water. It is indistinguishable from the neutral current interactions that occur between all species of neutrinos and electrons in normal water (b). In heavy water, neutrinos preferentially interact with nuclei. The electron neutrino's interaction now produces an electron in the final state (c) which is not seen in the neutral current interactions of all neutrino species with heavy water (d).

The SNO experiment has 3 phases. The first phase consisted of running with pure $\mathrm{D}_{2} \mathrm{O}$. For the second phase, $\mathrm{NaCl}$ was added to increase the mixture's neutron capture efficiency from $\sim 25 \%$ to $\sim 85 \%$. The final phase, running now, is again pure $\mathrm{D}_{2} \mathrm{O}$, but with ${ }^{3} \mathrm{He}$ proportional counters installed to measure the neutrons directly with an efficiency of $\sim 45 \%$. This phase has completely different systematics to the other phases and can be used as a cross check.

SNO's results for the first two phases show that the total ${ }^{8} \mathrm{~B}$ neutrino flux, as calculated by NC interactions, agrees very well with the existing solar models. However, there are fewer $\nu_{e} \mathrm{CC}$ interactions than expected (See Figure 3.5). If this is taken to be an indication of matter-enhanced oscillations occuring in the sun, the data favours a large mixing angle solution with oscillation parameters: 


$$
\Delta m_{12}^{2}=7.1_{-0.3}^{+1.0} \times 10^{-5} \mathrm{eV}^{2} \text { and } \theta_{12}=32.5_{-1.5}^{\circ+1.7},[18]
$$

Total Rates: Standard Model vs. Experiment Bahcall-Pinsonneault 2000

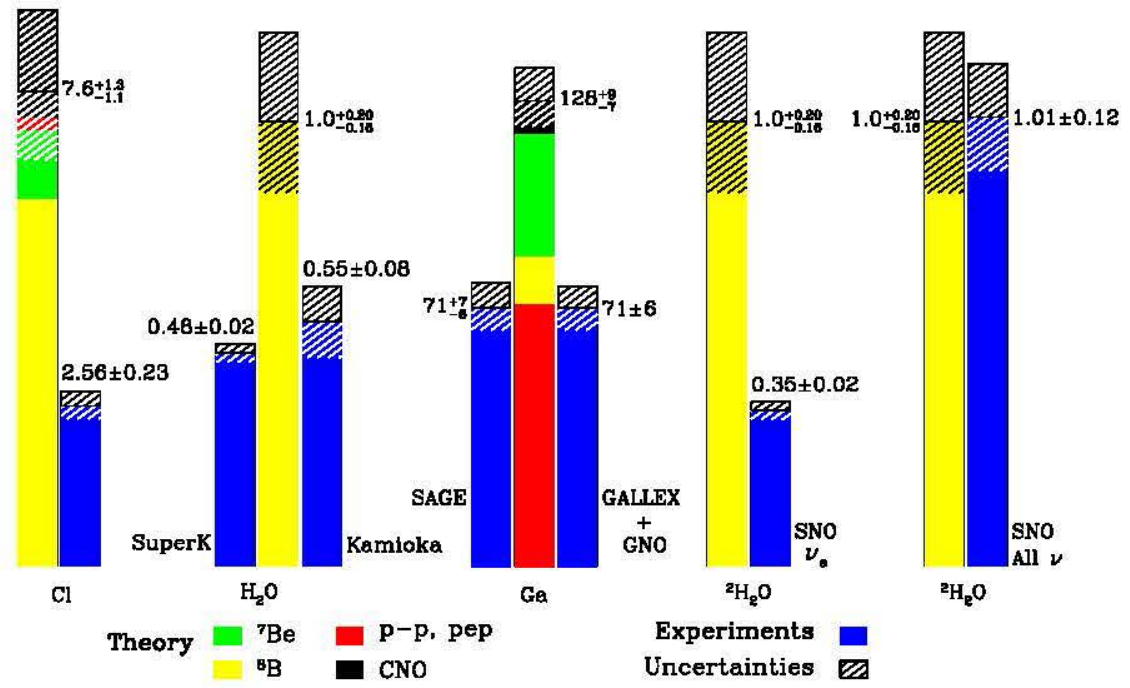

Figure 3.5: Comparison of predicted fluxes, from the solar model, to experimental measurements. The significance of the SNO result is that a deficit is observed when only electron neutrinos are considered, but good agreement between theory and experiment is seen when all neutrino flavours are taken into account.

\section{Atmospheric Neutrinos}

Atmospheric neutrinos are produced in decays of showers of muons, pions and other mesons that occur in the Earth's upper atmosphere as a result of cosmic ray interactions. A typical interaction sequence:

$$
\begin{aligned}
\pi^{ \pm} \rightarrow & \mu^{ \pm}+\nu_{\mu}\left(\overline{\nu_{\mu}}\right) \\
& \mu^{ \pm} \rightarrow e^{ \pm}+\nu_{e}\left(\overline{\nu_{e}}\right)+\overline{\nu_{\mu}}\left(\nu_{\mu}\right)
\end{aligned}
$$

is illustrated in Figure 3.6.

At the Earth's surface we therefore expect the ratio of $\nu_{\mu}+\overline{\nu_{\mu}}$ to $\nu_{e}+\overline{\nu_{e}}$ to be $2: 1$, to first order. 


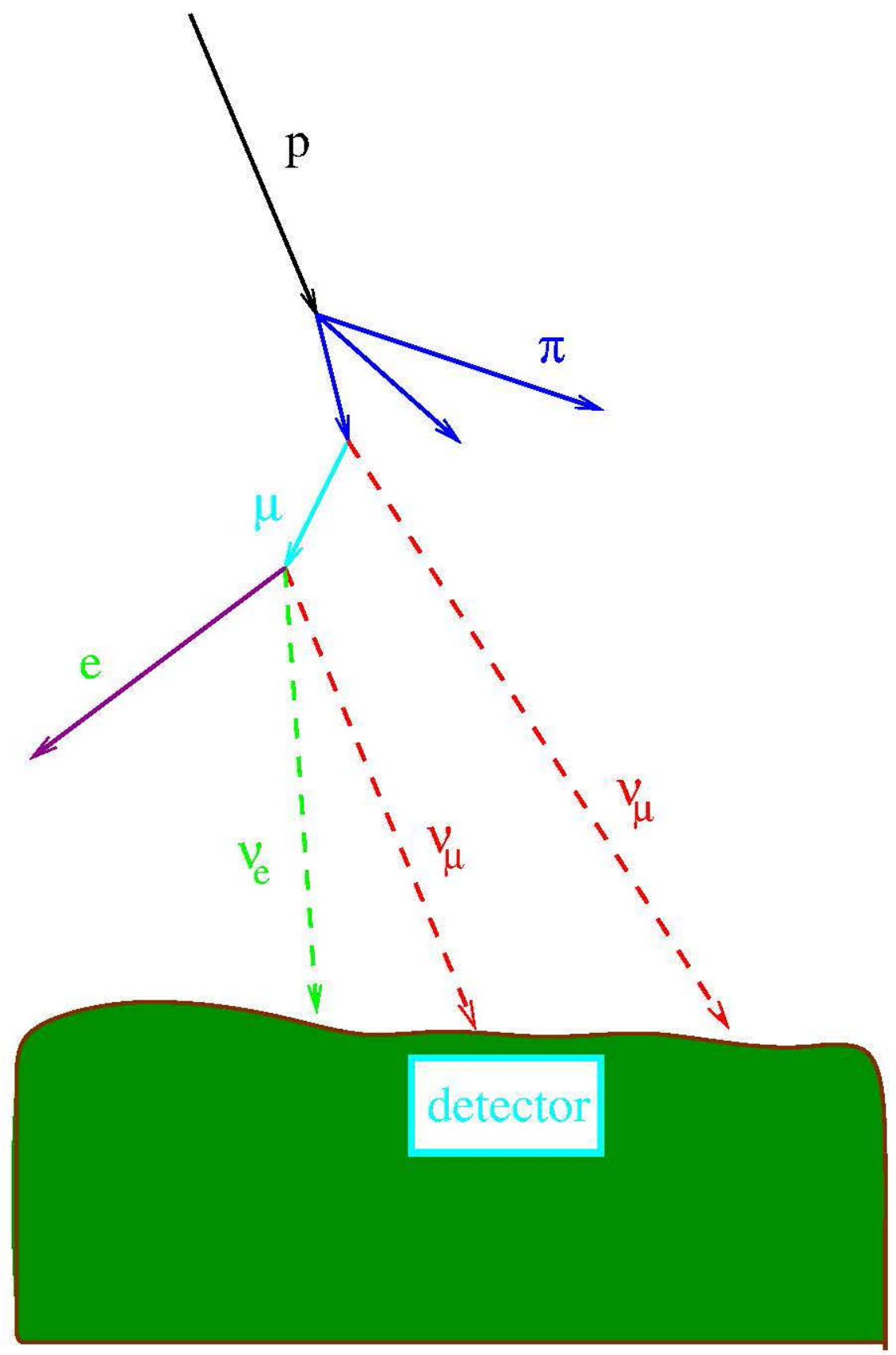

Figure 3.6: Cosmic rays incident on the upper atmospere interact with nucleons there, producing showers of secondary particles, primarily pions. These then decay to muons and muon neutrinos. The muons decay to muon neutrinos, electron neutrinos and electrons. 
Atmospheric neutrino detectors can measure the $\nu_{p} / \nu_{e}$ flux ratio by observing the final state leptons produced via CC interactions of neutrinos on nuclei. The flavour of the resultant lepton is used to identify the flavour of the neutrino that produced it. Experiments studying atmospheric neutrinos usually report their findings in terms of the ratio of ratios:

$$
\mathcal{R} \equiv \frac{\mathcal{R}_{D A T A}}{\mathcal{R}_{M C}}=\frac{\left(N_{\mu} / N_{e}\right)_{D A T A}}{\left(N_{\mu} / N_{e}\right)_{M C}}
$$

A $\nu_{\mu}$ deficit was first observed in 1983 in the IMB experiment $[69]$ and supported by Kamiokande [57] using large underground water Cerenkov detectors. Two experiments using iron sampling calorimeters, NUSEX ${ }^{13}$ [58] and the Fréjus experiment [59] did not observe this deficit, but later Soudan-II [60] and MACRO ${ }^{14}$ [61] confirmed it with higher statistics. The experiments converged on a value of $\mathcal{R} \sim 0.6$.

\section{Super-Kamiokande}

Super- $K$ is able to measure the direction and energy of charged particles in the detector by the Cerenkov rings produced in its $50 \mathrm{kT}$ of $\mathrm{H}_{2} \mathrm{O}$. By measuring the zenith angle and hence the distance the incident neutrino has travelled, oscillation hypotheses can be tested. The distances range from $10 \mathrm{~km}$ to greater than $10,000 \mathrm{~km}$ as can be seen in Figure 3.7. Super-K showed that their data exhibited a zenith angle dependent $\nu_{\mu}$ deficit whilst the $\nu_{e}$ spectrum was the same at all distances. Further data excluded $\nu_{\mu} \rightarrow \nu_{\text {sterile }}$ at the $99 \%$ confidence level, implying that the neutrinos that had travelled further through the earth had been more likely to undergo $\nu_{\mu} \rightarrow \nu_{\tau}$ oscillations. In a two flavour scheme, the oscillation parameters are determined to be:

$$
\Delta m_{23}^{2}=2.5_{-0.6}^{+0.5} \times 10^{-3} \mathrm{eV}^{2} \text { and } \sin ^{2} 2 \theta_{23}>0.9 \text { (90\% C.L.) }
$$

$\left(\theta_{23} \sim 45^{\circ}\right.$, maximal mixing $)$

\footnotetext{
${ }^{13}$ Nucleon Stability EXperiment

${ }^{14}$ Monopole, Astrophysics And Cosmic Ray Observatory
} 


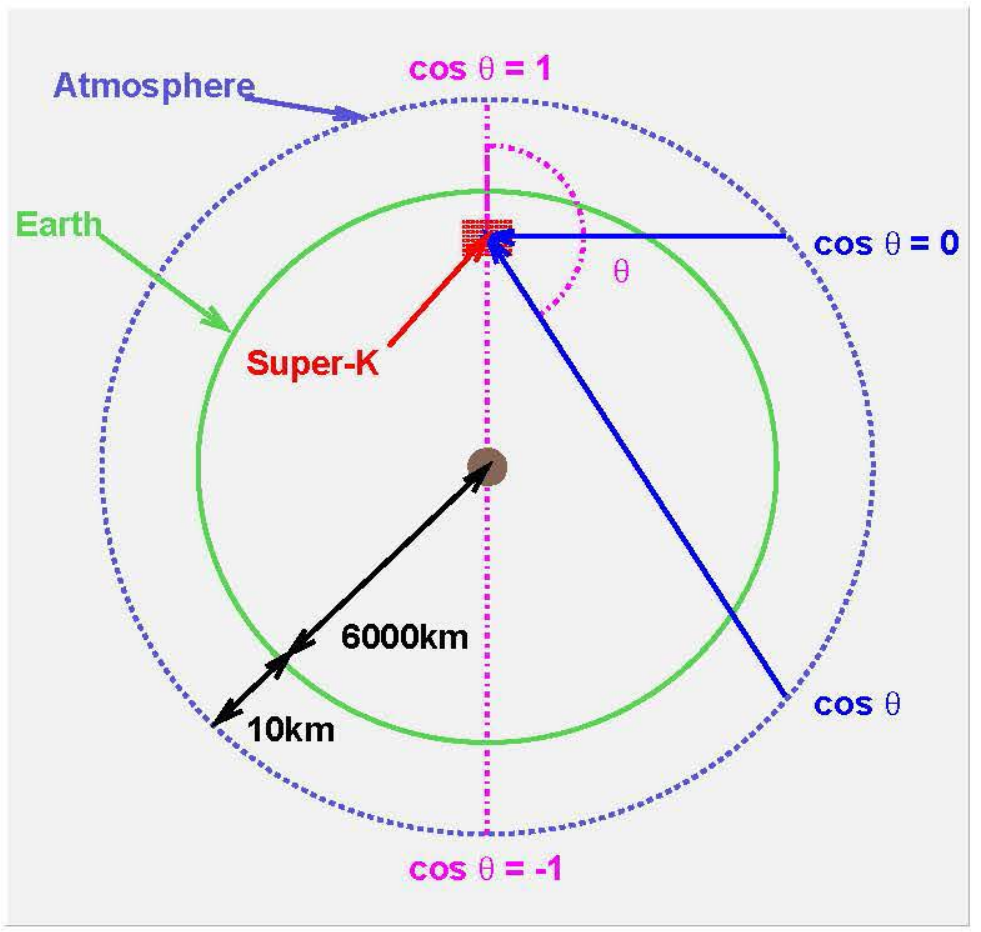

Figure 3.7: The distance an atmospheric neutrino has travelled varies with the zenith angle of its incidence. Super-K uses this dependence to test ascillation hypotheses.

\section{Reactor Neutrinos}

Nuclear reactors produce huge numbers of $\overline{\nu_{e}}$ during the fission of heavy nuclei such as ${ }^{235} \mathrm{U}$ and ${ }^{239} \mathrm{Pu}$ and they can be detected via the inverse beta decay interaction: $\bar{\nu}_{e}+p \rightarrow n+e^{+}$. Before 2002, the most sensitive reactor neutrino experiment was CHOOZ [64] which was located $\sim 1 \mathrm{~km}$ away from the reactor core of the CHOOZ power station in the Ardennes, Northern France. The experiment finished data taking in 1998 and found no evidence of spectral distortion after a full analysis, thereby excluding a large region of oscillation parameter space and strengthening evidence that $\nu_{\mu} \rightarrow \nu_{e}$ was not causing the atmospheric neutrino problem.

\section{KamLAND}

$\mathrm{KamLAND}^{15}$ is the first experiment to report evidence for reactor $\overline{\nu_{e}}$ disappearance [65]. Located in the site of the old Kamiokande experiment, KamLAND differs

\footnotetext{
${ }^{15}$ Kamioka Liquid scintillaotr Anti-Neutrino Detector
} 
from previous reactor based experiments in its extended baseline which arises from 26 reactors at distances of between 138 and $214 \mathrm{~km}$. This leads to $79 \%$ of the detectable neutrino flux travelling an average distance of $180 \mathrm{~km}$. The detector employs $1 \mathrm{kT}$ of liquid scintillator contained in a $13 \mathrm{~m}$ diameter spherical balloon which is read-out by PMTs. The gap between the sphere and the rock is flooded with water to create a Cerenkov detector which uses the old Kamiokande PMTs for readout and principally functions to tag cosmic muons. KamLAND's baseline enables it to probe smaller values of $\Delta \mathrm{m}_{12}^{2}$ than previous reactor experiments. KamLAND expected to see 365.2 events with no oscillations and observed 258 , yielding the oscillation parameters:

$$
\Delta m_{12}^{2}=7.9_{-0.5}^{+0.6} \times 10^{-5} \mathrm{eV}^{2} \text { and } \tan ^{2} \theta_{12}=0.40_{-0.07}^{+0.10}
$$

$\left(\theta_{12} \sim 32.3^{\circ}\right)$

The KamLAND and CHOOZ data combined also give the best limits on the third neutrino mixing angle, $\theta_{13}$ :

$$
\sin ^{2} \theta_{13} \leq 0.031[66]
$$

\section{Accelerator Neutrinos - Short Baseline}

Neutrino beams can be produced by firing high energy proton beams at targets, focussing the products and allowing them to decay. The advantage of this type of experiment over ones using natural neutrino sources is a greatly increased knowledge and control over the energy and flavour content of the neutrinos. Typical short baseline experiments have a detector up to $1 \mathrm{~km}$ away from the source with neutrino energies ranging from $10^{4}$ to $10^{10} \mathrm{eV}$. This results in oscillation sensitivities down to $\Delta \mathrm{m}^{2}=0.1 \mathrm{eV}^{2}$. The CERN based experiments CHORUS ${ }^{16}[70]$ and NO$\operatorname{MAD}^{17}[71]$ found no evidence for $\nu_{\mu} \rightarrow \nu_{\tau}$ oscillations and thus excluded $\Delta \mathrm{m}^{2}>1$ $\mathrm{eV}^{2}$. LSND ${ }^{18}[73]$ was a $\nu_{e}$ appearance experiment at Los Alamos which used a proton beam to produce a secondary beam of mostly $\pi^{+}$. Neutrinos are then produced

\footnotetext{
${ }^{16}$ CERN Hybrid Oscillation Research apparatuS

${ }^{17}$ Neutrino Oscillation MAgnetic Detector

${ }^{1.5}$ Liquid Scintillator Neutrino Detector
} 
via two processes:

$$
\begin{array}{ll}
\text { Pion decay in flight } & \pi^{+} \rightarrow \mu^{+}+\nu_{\mu} \\
\text { Muon decay at rest. } & \mu^{+} \rightarrow e^{+}+\nu_{e}+\overline{\nu_{\mu}}
\end{array}
$$

The $\nu_{e}$ are detected via $\nu_{e}+N u c l e o n \rightarrow e^{-}+X$. An excess of $\overline{\nu_{e}}$ is reported (detected via $\overline{\nu_{e}}+p \rightarrow e^{+}+n$ where a $2.2 \mathrm{MeV}$ photon arises from neutron capture) for both neutrino production processes, corresponding to $\overline{\nu_{p}} \rightarrow \overline{\nu_{e}}$ oscillations with $\Delta \mathrm{m}^{2} \sim 1 \mathrm{eV}^{2}$ and and $\sin ^{2} 2 \theta \sim 10^{-2}$. Other experiments have searched for $\nu_{e}$ appearance, such as $\mathrm{KARMEN}^{19}$ which was based at the Rutherford Laboratory in the UK. KARMEN was also a liquid scintillator detector, with a baseline of $17.5 \mathrm{~m}$. Contrary to LSND, no evidence of neutrino oscillations was found, excluding a large region of the LSND favoured parameter space.

The BooNE ${ }^{20}$ project, in which MiniBooNE is the first stage, was primarily designed to confirm or refute the LSND result. The MiniBooNE results are due in 2005 and should be able to exclude all of the LSND parameter space at $90 \%$ C.L.

\section{Long Baseline Accelerator Neutrino Experiments}

Long baseline neutrino experiments typically have distances from source to detector of several hundred kilometers. $\mathrm{K}_{2} \mathrm{~K}^{21} \quad[75]$ is an experiment based in Japan where a beam of $98 \% \nu_{p}$ is sent from the KEK accelerator facility to the Kamioka mine, $250 \mathrm{~km}$ away. There is a near detector at KEK to sample the unoscillated beam and the final beam measurements are made in the Super Kamiokande detector. The experiment aimed to observe $\nu_{\mu}$ disappearance between the two detectors, the average energy of the neutrinos being $1.3 \mathrm{GeV}$ which is not sufficient to allow an appreciable amount of $\nu_{\tau}$ appearance to occur. In 2002 , K2K claimed to have made an observation of neutrino oscillations [76] when a deficit of $\nu_{p}$ was observed at the far detector together with a distortion of the energy spectrum that is more consistent with oscillations than without. The probability that the results could be

\footnotetext{
${ }^{19}$ The KArlswahe Rutherford Medium Energy Neutrino Experiment

${ }^{20}$ Bonster Neutrino Experiment

${ }^{21}$ KEK to Kamioka
} 
explained by statistical fluctuations alone is less than $0.01 \%$. The best fit values for the $\nu_{\mu} \rightarrow \nu_{\tau}$ oscillation parameters are

$$
\Delta m_{23}^{2}=2.8_{-0.7}^{+0.4} \times 10^{-3} \mathrm{eV}^{2} \text { and } \sin ^{2} \theta_{23}=1
$$

which are consistent with the Super-K atmospheric neutrino measurements.

MINOS is another long baseline neutrino oscillation experiment which began taking oscillation data in January 2005. It is described in detail in Chapter 4.

CNGS $^{22}[62]$ is an experiment under construction which intends to send a $\nu_{p}$ beam $732 \mathrm{~km}$ from CERN in Switzerland to the Gran Sasso underground laboratory in Italy. The average neutrino energy will be $17 \mathrm{GeV}$, allowing $\nu_{\tau}$ appearance to be investigated. At Gran Sasso, there will be two large detectors, the OPERA ${ }^{23}$ lead/emulsion based detector and the ICARUS ${ }^{24}$ liquid argon TPC.

Further into the future, accurate measurements of $\sin ^{2} \theta_{13}$ are required. Two experiments that could address this are $\mathrm{T} 2 \mathrm{~K}^{25}$ and $\mathrm{NO} \nu \mathrm{A}^{26}$. Both experiments will use large detectors placed at some angle from the central axis of their respective accelerator $\nu_{\mu}$ beam. Although this leads to a lower event rate, the spread of neutrino energies seen is reduced. These experiments will look for $\nu_{e}$ appearance.

\footnotetext{
${ }^{22}$ CERN Neutrinos to Gran Sasso

${ }^{23}$ Oscillation Project with Emulsion-tRacking Apparatus

${ }^{24}$ Imaging Cosmic And Rare Underground Signals

${ }^{2 \pi}$ Tokai to Kamioka

${ }^{26} \mathrm{NuMI}$ Off-axis $\nu_{e}$ Appearance experiment
} 


\subsection{Summary}

A summary of the results for direct neutrino measurements are given in the table below:

\begin{tabular}{|c|c|c|c|c|c|}
\hline$\nu$ type & Mass limit & Experiment & Type & Year & Ref. \\
\hline$\overline{M_{\nu_{e}}}$ & $<2.2 \mathrm{eV}(95 \%$ C.L. $)$ & Mainz & ${ }^{3}$ He decay & 2000 & {$[33]$} \\
$\overline{M_{\nu_{k}}}$ & $<170 \mathrm{keV}(90 \%$ C.L. $)$ & P.S.I. & pion decay & 1996 & {$[35]$} \\
$\overline{M_{\nu_{\tau}}}$ & $<18.2 \mathrm{MeV}(95 \%$ C.L. $)$ & ALEPH & tau decay & 1998 & {$[17]$} \\
$\overline{M_{\nu_{e}}}$ & $<11 \mathrm{eV}$ & Kamiokande $/ \mathrm{IMB}$ & supernova & 1987 & {$[36]$} \\
$\sum m_{e, \nu, \tau}$ & $<0.7 \mathrm{eV}$ & WMAP $/ 2 \mathrm{dF}$ & sky survey & 2003 & {$[37]$} \\
\hline
\end{tabular}

Table 3.1: Direct neutrino mass measurements

Current limits from a selection of $0 \nu \beta \beta$ experiments follow:

\begin{tabular}{|c|c|c|c|c|c|}
\hline Experiment & Isotope & Half-Life (years) & Eff. Maj. Mass & Year & Ref. \\
\hline Heidelberg- & & & & & \\
Moscow & ${ }^{76} \mathrm{Ge}$ & $1.9-18.3 \times 10^{25}$ & $0.24-0.58 \mathrm{eV}$ & 2004 & {$[47]$} \\
IGEX & ${ }^{76} \mathrm{Ge}$ & $>1.57 \times 10^{25}$ & $<0.3-1.1 \mathrm{eV}$ & 2000 & {$[49]$} \\
Cuoricino & natural TeO & $\geq 7.5 \times 10^{23}$ & $\leq 0.26-1.4 \mathrm{eV}$ & 2004 & {$[51]$} \\
NEMO-3 & ${ }^{100} \mathrm{Mo}$ & $>4.6 \times 10^{23}$ & $<0.7-2.8 \mathrm{eV}$ & 2004 & {$[63]$} \\
NEMO-3 & ${ }^{82} \mathrm{Se}$ & $>1.0 \times 10^{23}$ & $<1.7-4.9 \mathrm{eV}$ & 2004 & {$[63]$} \\
\hline
\end{tabular}

Table 3.2: Mass measurements from $0 \nu \beta \beta$

The data can also be summarized visually in oscillation parameter space. Figure 3.8 shows the dominant $\nu_{\mu} \leftrightarrow \nu_{\tau}$ oscillations and Figure 3.9 shows a zoom in to the region favoured by a combined fit of the K2K and Super-Kamiokande experiments. Figure 3.10 shows the $\nu_{\mu} \leftrightarrow \nu_{e}$ parameter space excluded and allowed by various experiments. The so called 'Small Mixing Angle' (SMA), 'Large Mixing Angle' (LMA), 'Low $\Delta \mathrm{m}^{2}$ ', (LOW) and 'Quasi-Vacuum' (VAC) solutions are 


\begin{tabular}{|c|c|c|c|}
\hline Parameter & Main Sources & Best Fit & $\mathbf{2} \sigma$ \\
\hline$\Delta m_{12}^{2}\left(10^{-5} \mathrm{eV}^{2}\right)$ & KamLAND, SNO & 7.9 & $7.3-8.5$ \\
$\Delta m_{23}^{2}\left(10^{-3} \mathrm{eV}^{2}\right)$ & Super-K, K2K & 2.2 & $1.7-2.9$ \\
$\operatorname{Sin}^{2} \theta_{12}$ & KamLAND, SNO & 0.30 & $0.25-0.34$ \\
$\operatorname{Sin}^{2} \theta_{23}$ & Super-K, K2K & 0.50 & $0.38-0.64$ \\
$\operatorname{Sin}^{2} \theta_{13}$ & KamLAND, CHOOZ & 0.0 & $\leq 0.031$ \\
\hline
\end{tabular}

Table 3.3: Neutrino oscillation parameters from various experiments.

shown. Figure 3.11 shows that a combined analysis of solar experiments and KAMLand strongly disfavours all solutions except LMA, in which three islands become pronounced. The LMA-1 solution is the best fit to all data available.

Three different $\Delta m^{2}$ and 4 neutrinos are required to accomodate all the data since $\Delta m_{S O L}^{2} \ll \Delta m_{A T M}^{2} \ll \Delta m_{L S N D}^{2}$. However data from LEP shows that there are only 3 light neutrinos meaning that a fourth would be sterile, a hypothesis that is strongly disfavoured by oscillation experiment data. The results of miniBooNE should clarify the situation. 


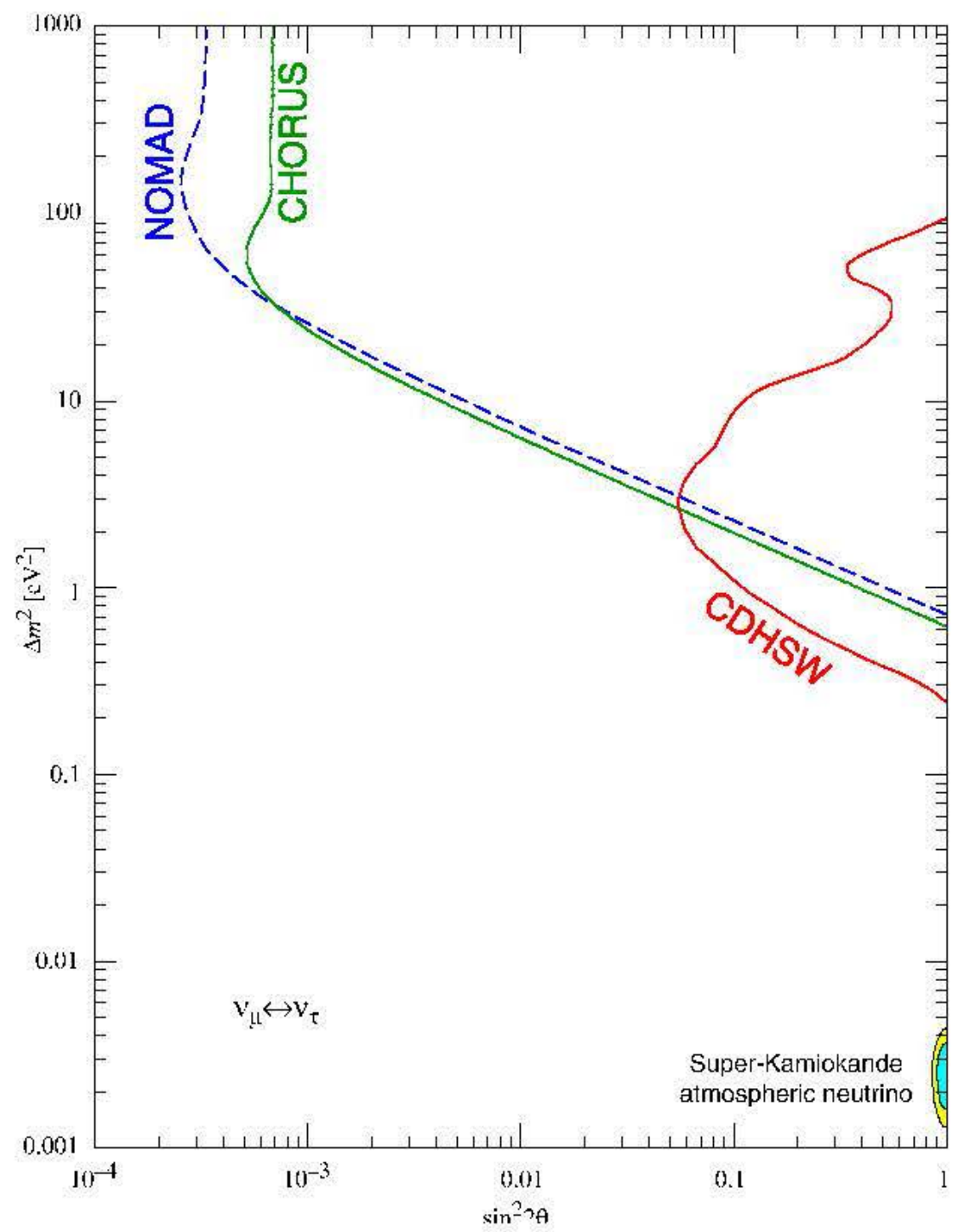

Figure 3.8: The allowed and exeluded regions in parameter space for $\nu_{\mu} \leftrightarrow w_{r}$ osedilations. The exeluded regions arise from short baseline experiments wr appearanee experiments at CERN. The allowed region is determined by Super-K data. 


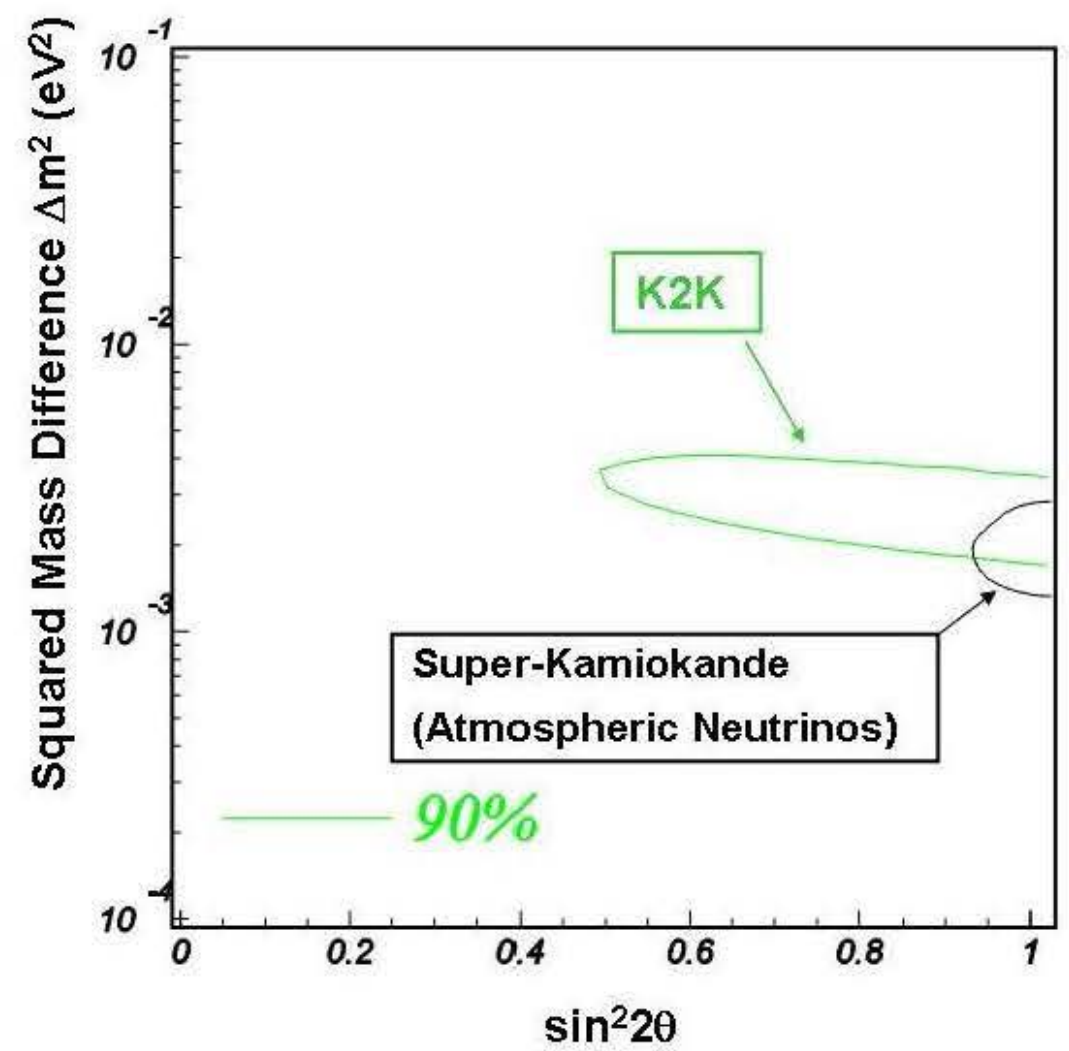

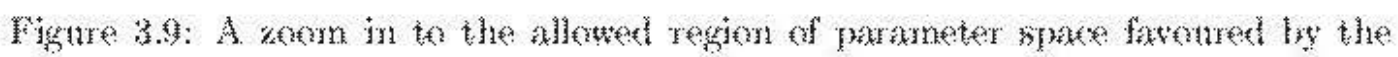
Supexw- ant 


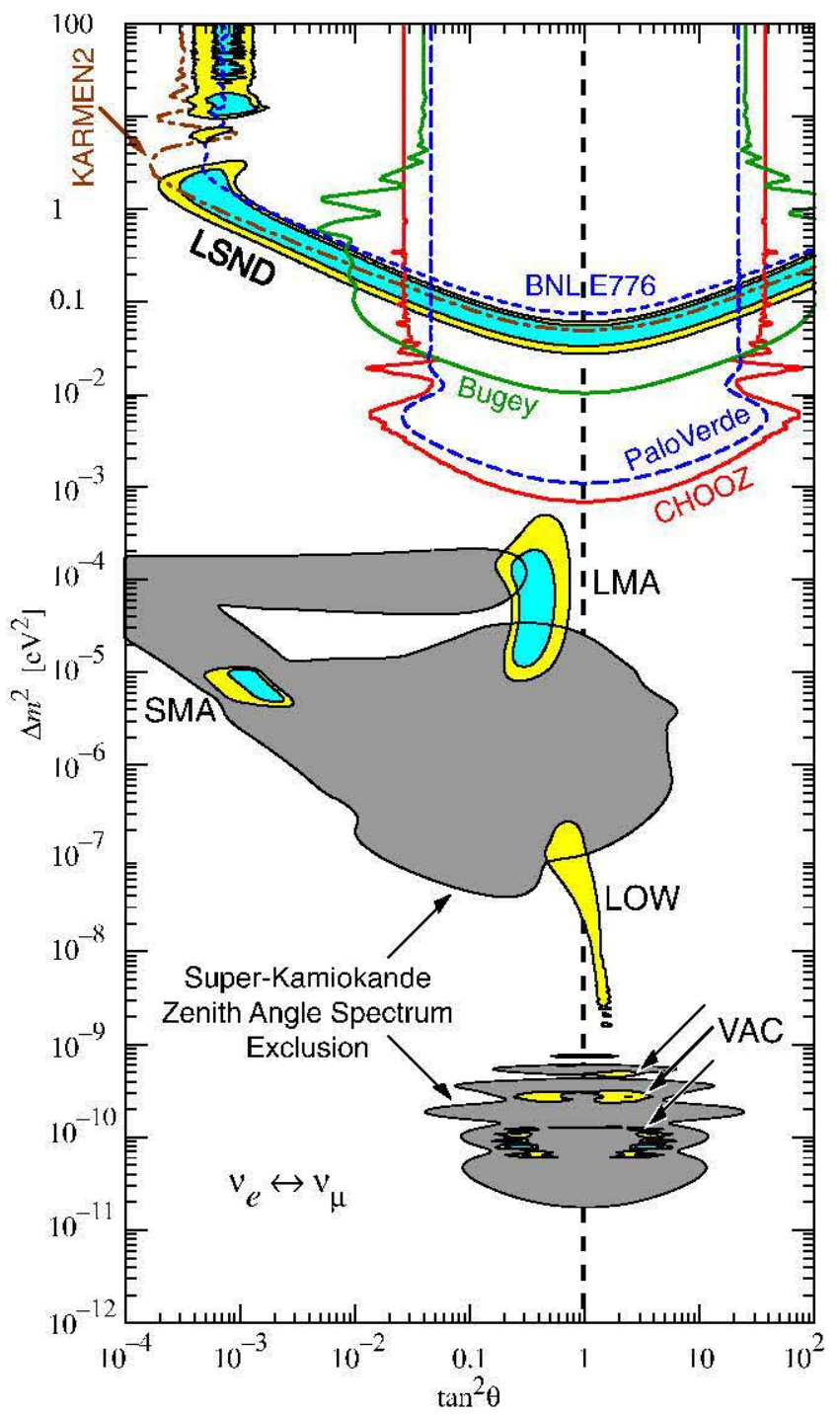

Figure 3.10: The allowed and excluded regions of parameter space for $\nu_{p} \leftrightarrow \nu_{e}$ oscillations. The excluded regions from KARMEN and BNL are from $\nu_{e}$ appearance as is LSNDs preferred region. The CHOOZ, Bugey and Palo Verde excluded regions are all based on $\overline{\nu_{e}}$ disappearance experiments. The preferred solar regions are marked $\mathrm{LMA}^{31}, \mathrm{SMA}^{32}, \mathrm{LOW}^{33}$ and $\mathrm{VAC}^{34}$ and are derived from $\nu_{e}$ disappearance at Homestake, SAGE, GALLEX, GNO, Super-K and SNO. The excluded region from Super-K's zenith angle spectrum is also shown. 


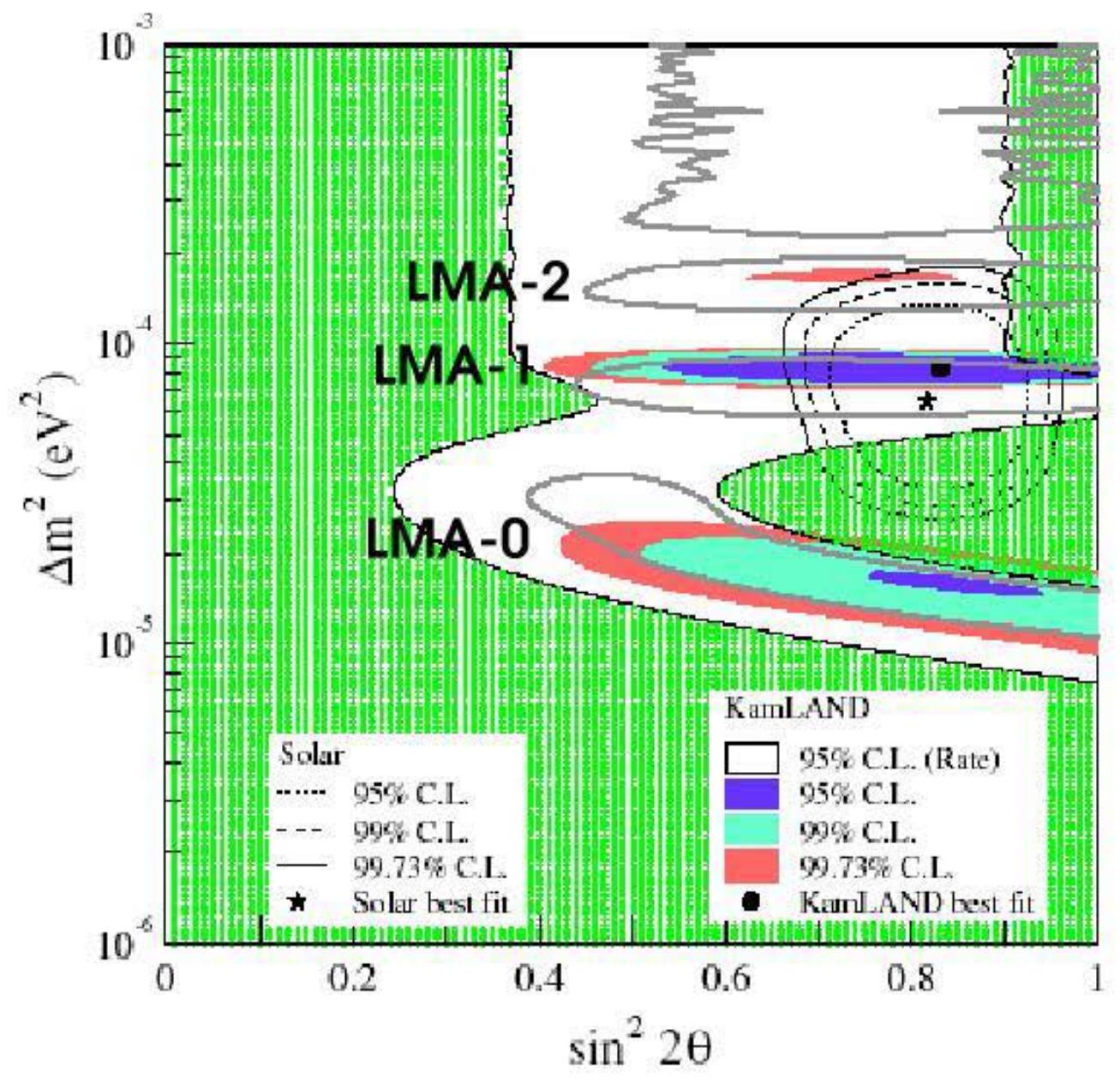

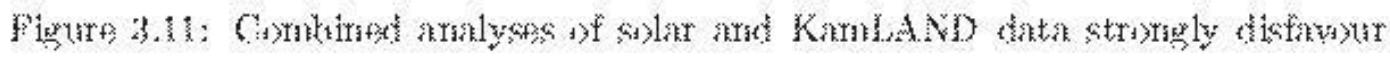

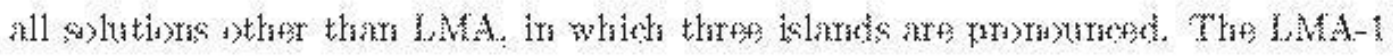

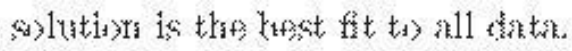




\section{Chapter 4}

\section{The NuMi-MINOS Experiment}

\subsection{Introduction}

MTNOS stands for Main Injector Nentrimo Oscillation Search. It is a long baselme experiment where nentrinos travel $730 \mathrm{~km}$ between two detectors.

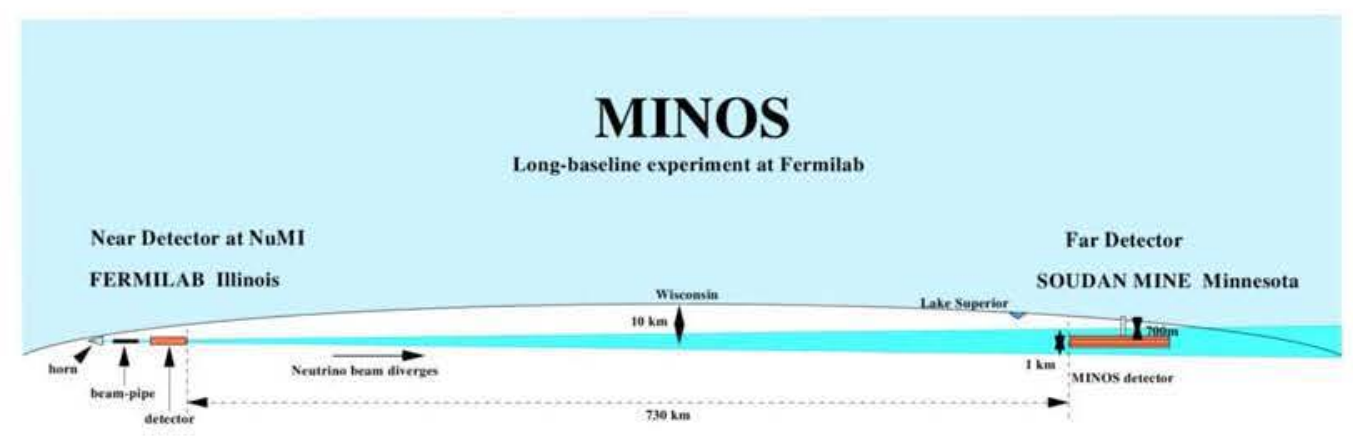

Figme 4.1: The path the NuMI bean takes from Fermilab to the Far Detector in the Somtan Mime in Mimesota. The beam reaches a maximm depth in the Earth of aromi lokm and passes completely muderneath Lake Superior.

The principle of the experiment is to generate a beam of mostly mon nentrinos at the Main Injector at Fermilab, send the bean through the "Near Detector" at Fermilab where the mentrino spectrm is sampled and them allow the bean to pass through the earth mutil it reaches the 'Far Detector' in Mimesota where it is measmed again. The detectors are designed to be as similar as possible to reduce systematic effects, but certain issues mean that it is not cost effective for them to 


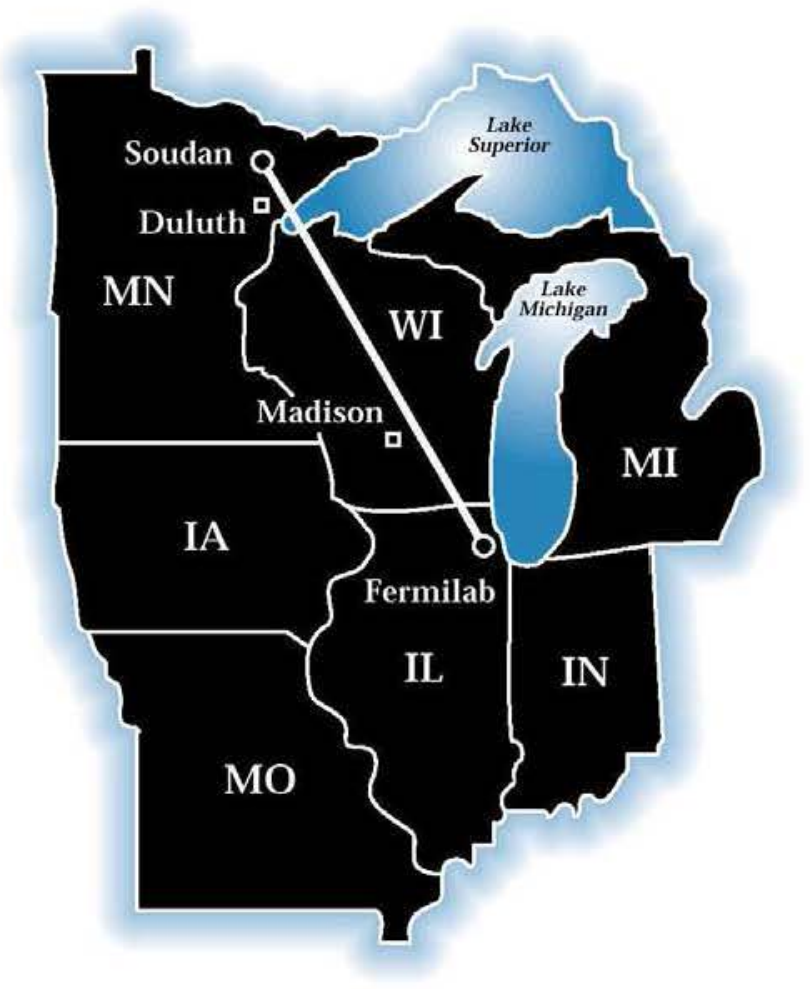

Figme 4.2: The beam is generated at Fermilab in llimois, crosses mulermeath Wiscomsin and is detected at the Far Detector in the Somban Mine Mimesota.

be irentical.

\subsection{NuMI}

The Nentrimos at the Mam Injector beam is created at Fermilab. A particle shower of mostly pions and kafms is produced when a 120 GeV beam of protons is incident mpon a carbon target. The beam has been online since December 2004 and at peak performance, the matim injector is expected to deliver $2.5 \times 10^{13}$ protons on target per 8.7 fusecond spill.

The charged particles are then focussed by two parabolic magnetic horns down a 675 m evacuated decay pipe in which the hadrous decay to mons and mum nemtrinos. Having passed through the pipe, any remaining hadrous are stepped by a water-moled hatron absorber and $240 \mathrm{~m}$ of Dolomite rock removes the mums from the nentring beam. $50 \mathrm{~m}$ past this lies the Near Detector. Figure 4.3 illustrates the 
beam production profess. The beam is pointed $3.3^{\circ}$ downwats to take into afount the curvature of the earth that has occurred by the time the beam reaches the far Detextor.

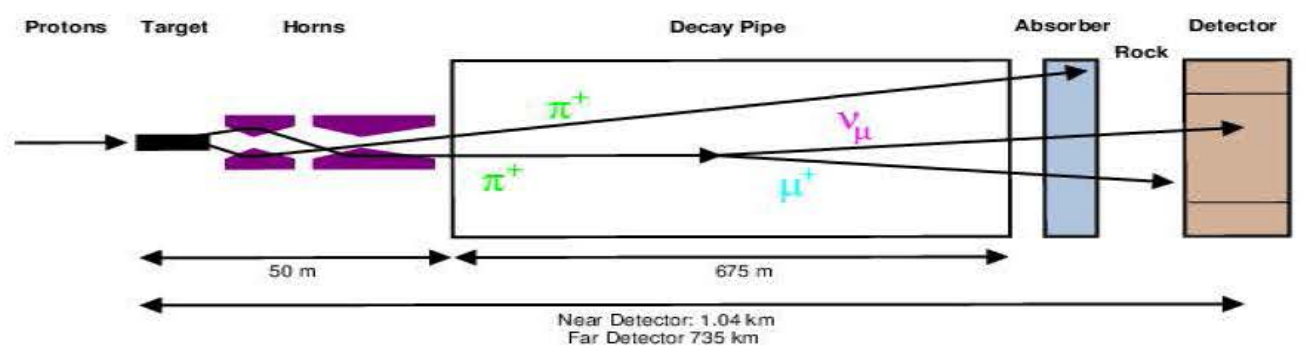

Figure 4.3: The lavout of the structures that are wed to create a neutrino beam from a proton sourse. The protons are incident on atarget and the resulting pion shower is focussed by two magnetic hom apparatuses. The pions decay to mons and mon neutrinos in the deaty pipe section and any remaining hatrons are absorbed in the water cooled absorber and rock.

The relative positions of the target and homs can be adjusted, which has the effext of changing the energy spextra of the neutrinos producel. It has been proposed that at any given time, the beam be set to one of three distinct configurations, know as the High, Metium and Low energy modes. Figure 4.4 shows the resutant spectrat at the Thr Detector of these modes.

Recent results, manly from Super-Kamiokande, indicte that $\Delta \mathrm{m}^{2}$ is sman which makes the low energy option the best for MINOS. Ruming will largely be in this monle although the event rate will suffer as a radt.

One of the major soures of uncertainty in the MNOS experiment is catsed by the extrapatation of the neutrino spextrum from the Near to the far Detetors. This is befause the initial hadron content and decay kinematis are not fuly understood. To address this, a separate experiment has been set up at Fermiab called MTPP Main Injector Particle Production. This experiment will study the particles profuced from a proton beam incident on the MINOS carbon target. The results fan be we to refine Monte Carlo simulations. 

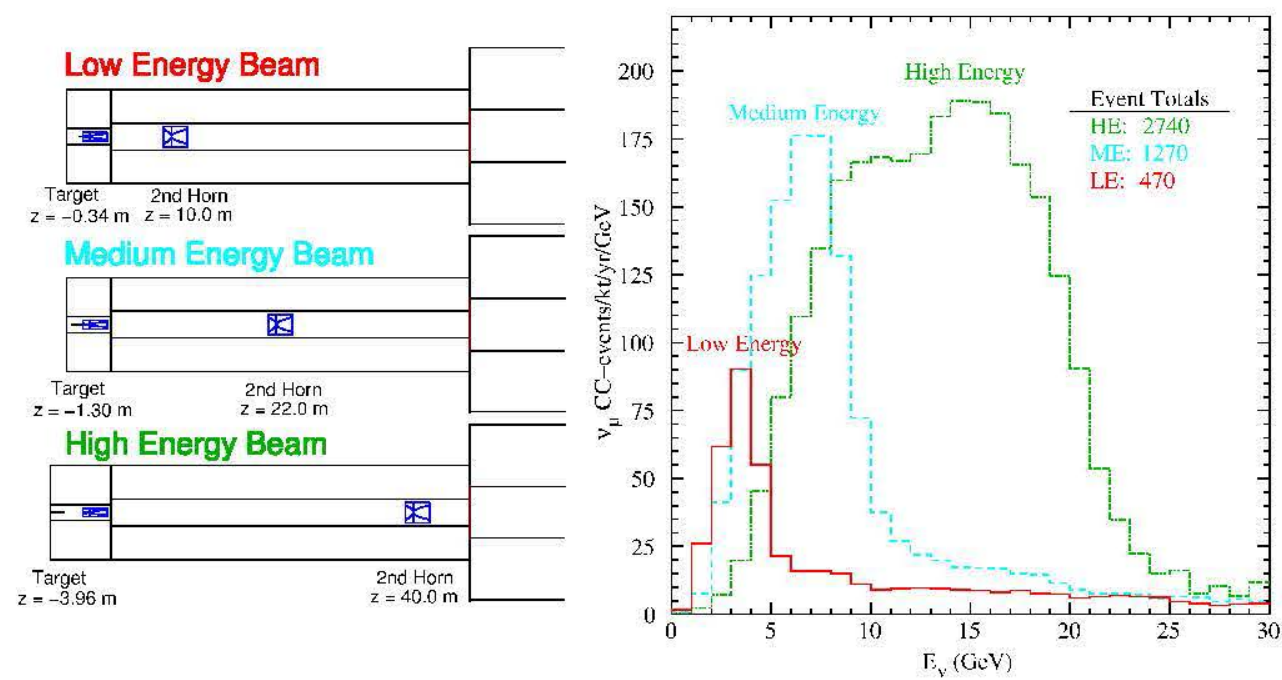

Figure 4.4: The three possible configurations of the NuMI beam. On the left is the target and hom arrangement for the low, medium and high energy beams. On the right is the anticipated $\nu_{\mu}$ CC Far Detector energy spectra, in the case where there are no oscillations, for each of the beam options. MINOS will take data primarily in the low energy configuration.

\subsection{General Design of the MINOS detectors}

All of the MINOS detectors are based around a muon spectrometer design, using alternating planes of steel $(2.54 \mathrm{~cm}$ thick) sandwiched to as many as 192 scintillator strips (4.2 $\mathrm{x} 1 \mathrm{~cm}$ thick) to measure the energy loss of particles as they travel through the detectors. There is also a $6 \mathrm{~cm}$ air gap between planes. Individual scintillator strips traverse the entire width of the plane, so alternating planes have their strips oriented at $90^{\circ}$ to each other in order that a full 3D reconstruction of particle tracks is possible. This translates to a detector resolution of $\sim 23 \% / \sqrt{E}$ for electromagnetic showers and $\sim 55 \% / \sqrt{E}$ for hadronic showers.

The scintillator itself is made of polystyrene doped with fluor (PP0, 1\%) and POPOP, $(0.030 \%)$ a wavelength shifting compound. Each strip has a groove into which a $1.2 \mathrm{~mm}$ Kuraray wavelength shifting (WLS) fibre is inserted. This allows the light produced in the scintillator to be transported to Hamamatsu photomultiplier tubes (PMTs) in order to be read-out. 
Both detectors are magnetised in order to separate positively and negatively charged particles and to allow particles' energies to be determined from their curvature. This method is most effective at high energy; at $10 \mathrm{GeV}$ a muon's momentum can be measured to $\sim 14 \%$. At lower energies a resolution of $\sim 6 \%$ can be achieved by measuring the range of the muon in the detector. [90]

The detector channels are calibrated using charge injection on the front-end boards and light injection into the strips via pulser boxes. These send UV light pulses to all of the strips via a manifold and can be used to monitor the gain of the PMTs over time. Strip-to-strip calibration is achieved by tracking cosmic ray muons as they traverse the detector. The PMTs are powered by LeCroy 1440 high voltage supplies.

\subsection{The Near Detector}

The purpose of the Near Detector is to act as a zero reference point for the Far Detector. The total mass of the detector is 980 tonmes, most of which comes from 282 planes of steel. Each plane is $3.8 \mathrm{~m}$ high and $4.8 \mathrm{~m}$ wide. The detector is divided into 4 sections or regions (See Figure 4.5) which are intended to fulfil specific roles whilst minimising the overall size and cost of the detector.

The first section, the veto region exists to exclude neutrons and end effects from events that will be considered for analysis. The next section is the target region. Interactions occuring here will be the ones used for comparison with the Far Detector. The shower region is designed to be large enough to fully contain hadronic showers produced by neutrino interactions in the target section. The final section is the muon spectrometer which is designed to be large enough to allow muons to run out of energy and stop or for sufficient curvature to occur in order that the muon's momentum can be determined.

The Near Detector has been designed such that particles travelling through it experience a similar field to that which they would at the Far Detector. However the beam spot clearly cannot be in the same place as the field coil hole as a large proportion of events would be lost. 


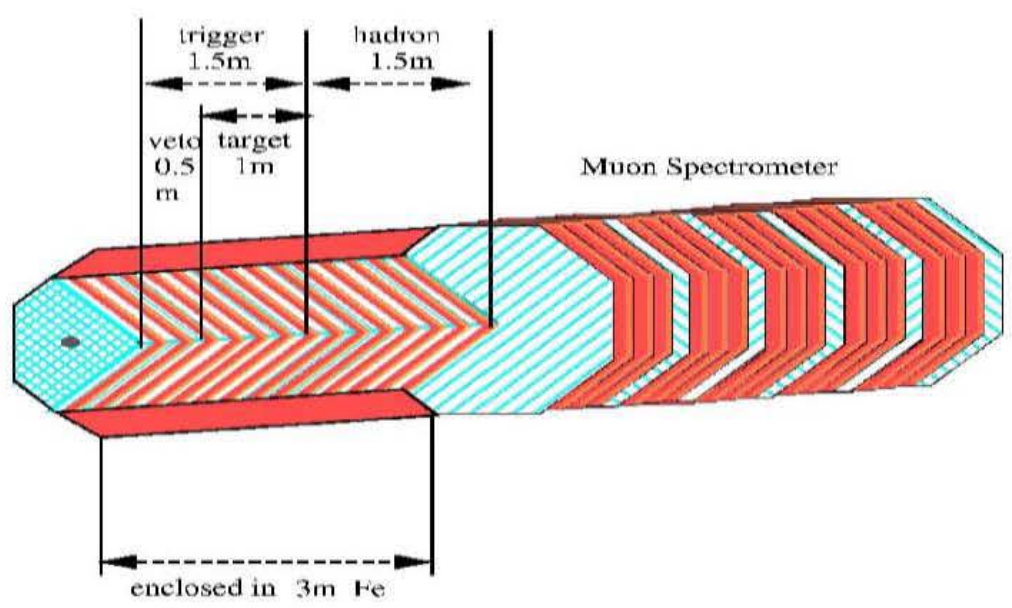

Figure 4.5: The instrumented regions of the Near Detector. The distances given are in terms of thickness of steel, and not actual length of detector. See Table 4.1 for details of the composition of the various detector regions. Steel is shown in red and scintillator in blue.

\begin{tabular}{|c|c|c|c|}
\hline Name & Length $(\mathbf{m})$ & No. Planes & Intrumentation Type \\
\hline Veto & 0.5 & 20 & 1 \\
\hline Target & 1 & 40 & 1 \\
\hline Hadron Shower & 1.5 & 60 & 1 \\
\hline Muon Spectrometer & 4 & 160 & 2 \\
\hline
\end{tabular}

Table 4.1: A description of the four parts of the Near Detector, in order starting with the first part to see the beam. Intrumentation Type 1 indicates that one in every five planes is fully instrumented with the other four planes being partially instrumented. Type 2 indicates that one plane in every five planes is fully instrumented with the other four planes being uninstrumented.

In order to satisfy both these conditions, all sections are constructed in the same shape; octagonal with a width greater than its height. The beam spot is at $50 \mathrm{~cm}$ to the left of the centre of each octagon and the coil hole is $50 \mathrm{~cm}$ to the right of the centre. The beam spot has a radius of about $25 \mathrm{~cm}$ so few events should be lost and magnetic field is $1.5 \mathrm{~T}$, as at the Far Detector. The small beamspot and 
the fact that the tramsverse spread of hadronic showers is only 50 cm means that a comsiderable saving in cost for a small reduction in resolution can be acheved by partially instrmmenting the detector. In the first thee detector regions, only one in every five planes is fully instrmented. The other planes are 'partially' instrumented. that is they have one 'unatrant' of scintillator, which is a region a little over a (unarter of me plane (See Figure 4.6). In the final section of the detector, the muon spectrometer, every fifth plane is fully intrmented with every other plane being completely minstrmented. Here a mom's cmvatme determines its momentmm; the measmement camot be improved with higher detector gramiarity.
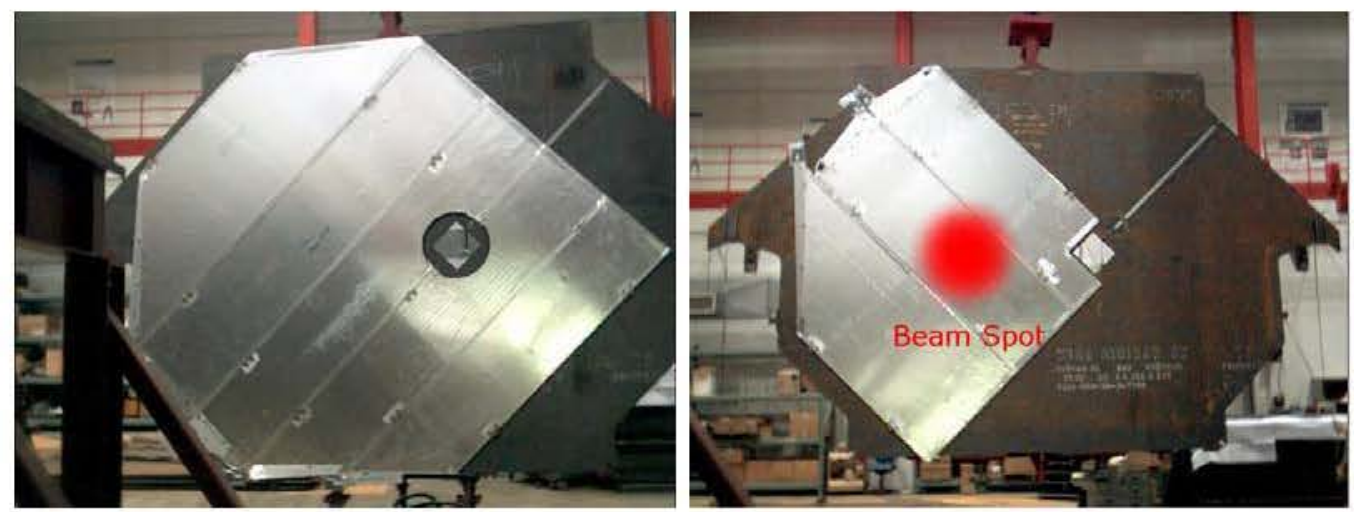

Figure 4. 6: On the left is a fuly instrmmented Near Detector plane. It is composed of a suashed octagen of steel with five scintillator modules attached. On the right is a partially instrumented plane composed of steel and thee scintillator modules. The positima on the beam spot is also shown.

The detector is readont at me end of the strip. The other end has a mylar surface which reflects light back to the read-ont. 64 pixel PMTs (M64s) are used to deted the scintillation light produced in the strips. The event rates are expected to be large in the Near Detector, so high speed QTE electronics are used for the reath-out. This fom of read-out is deadtime-less, uses a multi-range ADC system and divides the signal seen inte loms time 'buckets' which are digitised separately. 


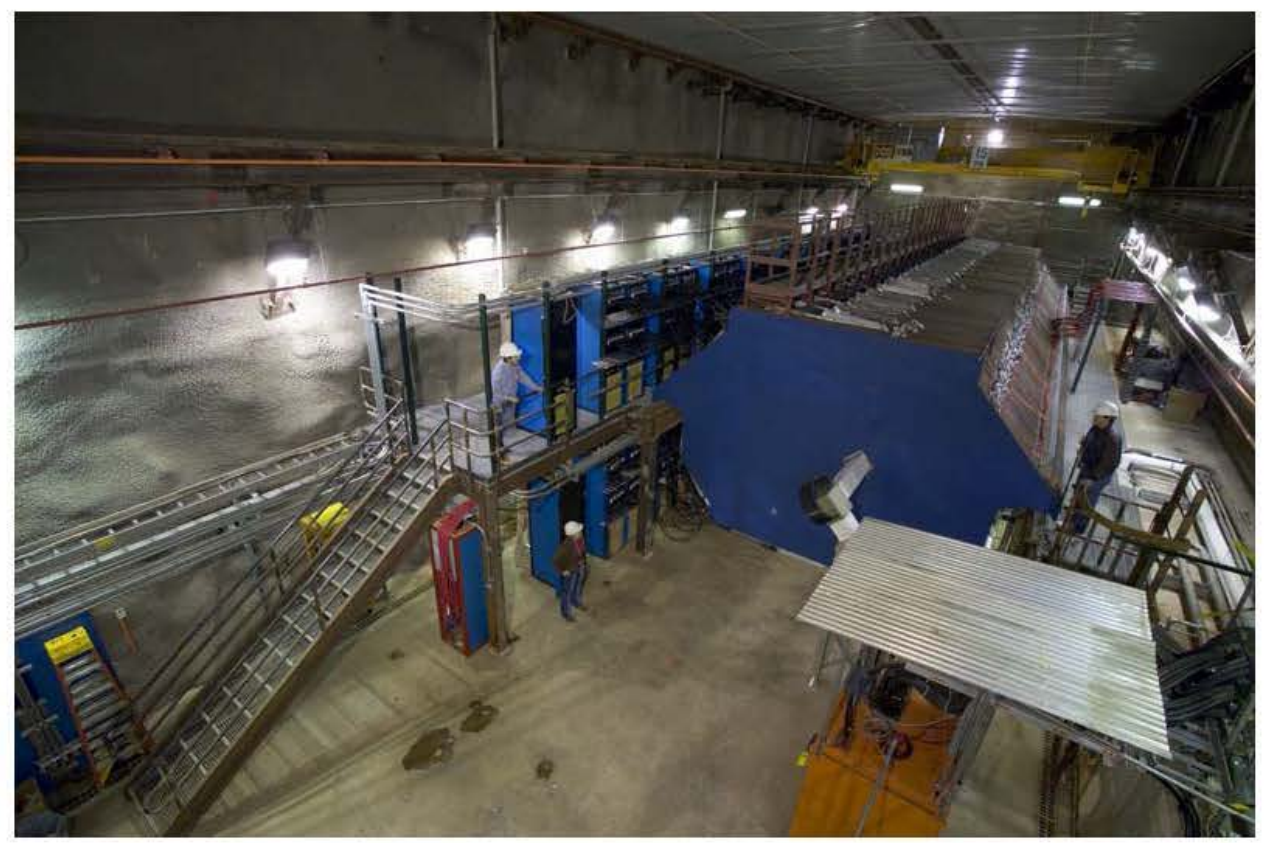

Figure 4.7: The Near Detector fully installed underground at Fermilab.

\subsection{The Far Detector}

The Far Detector is situated in the Soudan Mine, Minnesotas at a depth of $710 \mathrm{~m}$ (2100 meters water equitalent). It weighs $5.4 \mathrm{k}^{\prime} \mathrm{T}$, most of which comes from the 486 iron and scintillator planes that each have a diameter of $8 \mathrm{~m}$. The detector is divided into two 243 plane 'supermodules' which each have a $15 \mathrm{kA}$-furn coll rumning through their centres to provide a $1.5 \mathrm{~T}$ magnetic field. The two phase construction allowed cosmic ray data taking to commence with hat of the detector whilst the ofher hat was being constructed. Routine data faking with the fully operational detector has now been laking place since August. 2003.

Each of the scintillator planes is composed of 192 strips. They are read-out at both ends by 16 pixel M16 PMTs. As a rexult of the low event rate at the Far Detector, a procedure known as multiplexing has been used to reduce the amount of readont electronics required. Each pixel on the PMT is connected to the WLS fibre from 8 separate strins, selected such that the distance between them is maximised (See Figure 4.9) Sot ware then 'de-muxes' the read-out by utilising the act that the two ends have different multiplexing schemes which lead to a unique solution. 


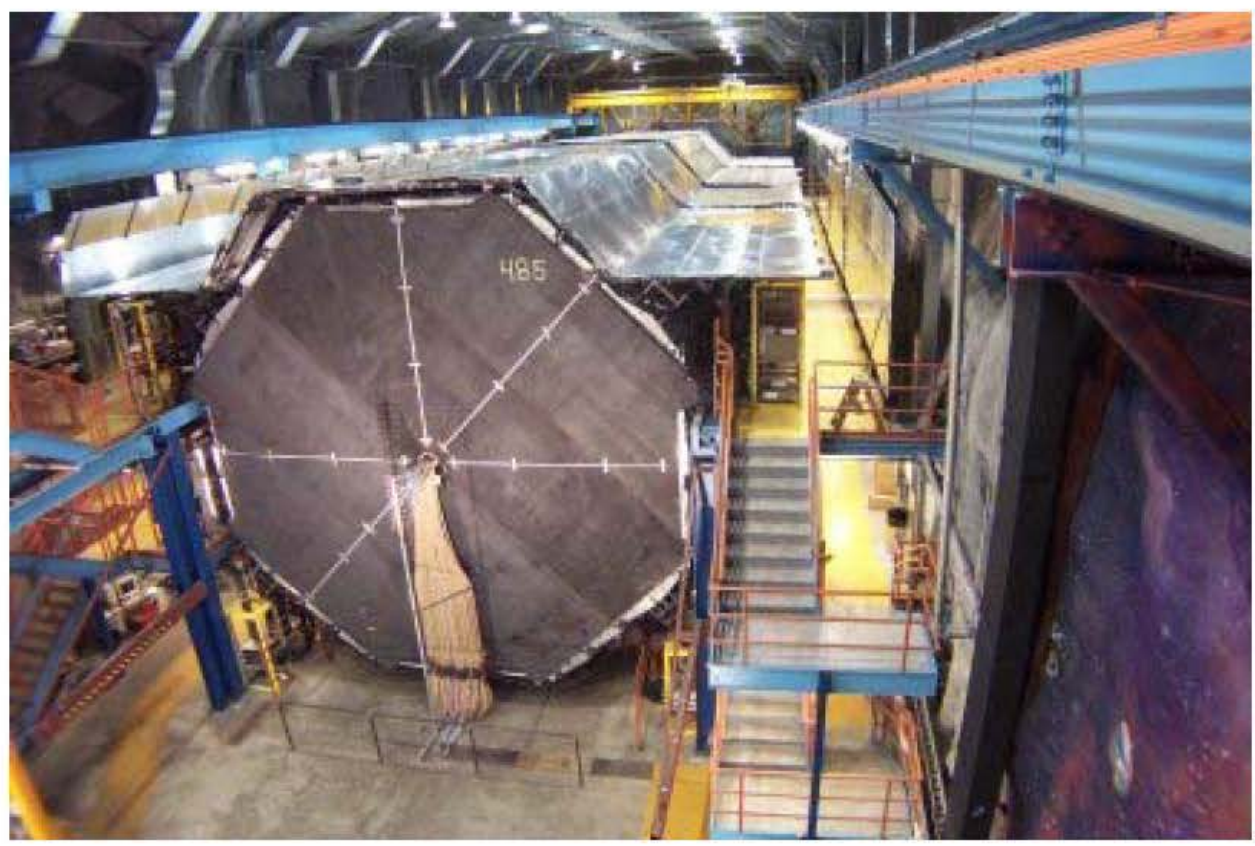

Figure 4.8: The fully installed Far Detector underground at the Soudan Mine, Minnesota. The last plane (furthest from Femilab), the magnetic coil (centre) and the veto shield (top) are clearly visible.

This 8-10-1 multiplexing generates a considerable cost saving in PMTs and read-out electronics.

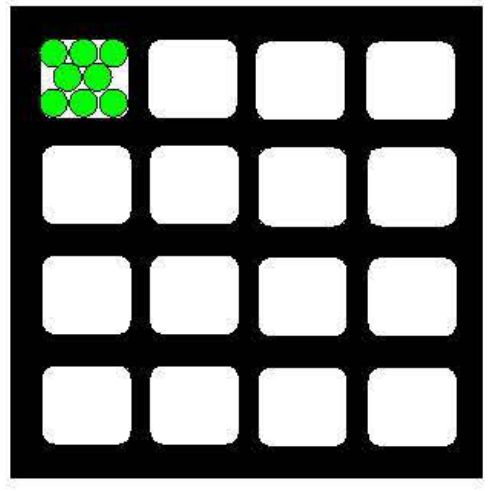

Figue 4.9: Diagram showing the layout of spot positions, overlaid on one pixel of an M16. There is no equivalent for M64s as the Nexa Detector does not use multiplexing.

The PMTs are read-out by a modified Viking chip known as a VA chip. VME crates pass the data to the data acquisition system (DAQ). This system does have 
deadtime but event rates are low in the Far Detector.

A relatively recent modification to the design of the Far Detector has been the inclusion of the veto shield. This is two additional layers of scintillator placed above and around the upper portion of the detector. This greatly reduces the number of 'false' neutrino events seen that are caused by muons entering the detector at a very steep angle and travelling a considerable distance in the air gap between planes before interacting with scintillator. Events of this type now produce tell tale hits in the veto shield. In addition, timing information from the veto shield can be used to determine whether and incoming muon was a down-going cosmic ray muon or an up-going 'rock' muon. 


\subsection{The Calibration Detector}

The third MINOS detector is the calibration detector, or CalDet.

The CalDet is a greatly scaled down version of the other MINOS detectors. It is composed of $601 \mathrm{~m} \times 1 \mathrm{~m}$ steel-scintillator planes and weighs approximately 12 tomnes. Each steel plane is $2.50 \mathrm{~cm}$ thick and the scintillator is split up into $24 \mathrm{x}$ $4.1 \mathrm{~cm}$ wide strips. The strips of the planes are oriented alternately horizontally and vertically with respect to the ground in order to achieve 3 dimensional reconstruction. This differs from the near and far detectors' strips which are also oriented at $90^{\circ}$ to each other in successive planes but at $45^{\circ}$ to the ground. CalDet's size lent it a great deal of flexibility, which allowed various configurations of cable length and read-out to be implemented (See Table 4.2). CalDet was not magnetised, unlike the other detectors.

There were a number of goals behind the ruming of the CalDet. Firstly, it was the first opportunity to ensure that the separate parts of the detector would work together as an integrated system. The Light Injection (LI) and cosmic ray calibration procedures could be validated and optimised. The electronic and hadronic responses of the detectors could be determined in a particle beam of known energy. This is a crucial part of the energy scale determination for the entire MINOS experiment. Lastly, the near and far electronics systems could be compared to one another.

CalDet was used for 3 years in a series of test beams at CERN. For the first two years, far detector electronics were used almost exclusively and in the final year a combination of near/far and near only electronics were used. $6 \mathrm{~m}$ clear optical fibre was used at one end to simulate the read-out cables at the far detector and various lengths of green fibre were used at the other end to simulate different light path lengths. During near only running, specialised reflector connectors were used to return the light to one end as is the case with the near detector. This meant there was no read-out on the other end.

Originally, the CalDet electronics were intended to run in dynode trigger mode, as with the Far Detector. In this mode, the front-end electronics begin digitization when the dynode signal from a PMT goes over a certain threshold, typically one 


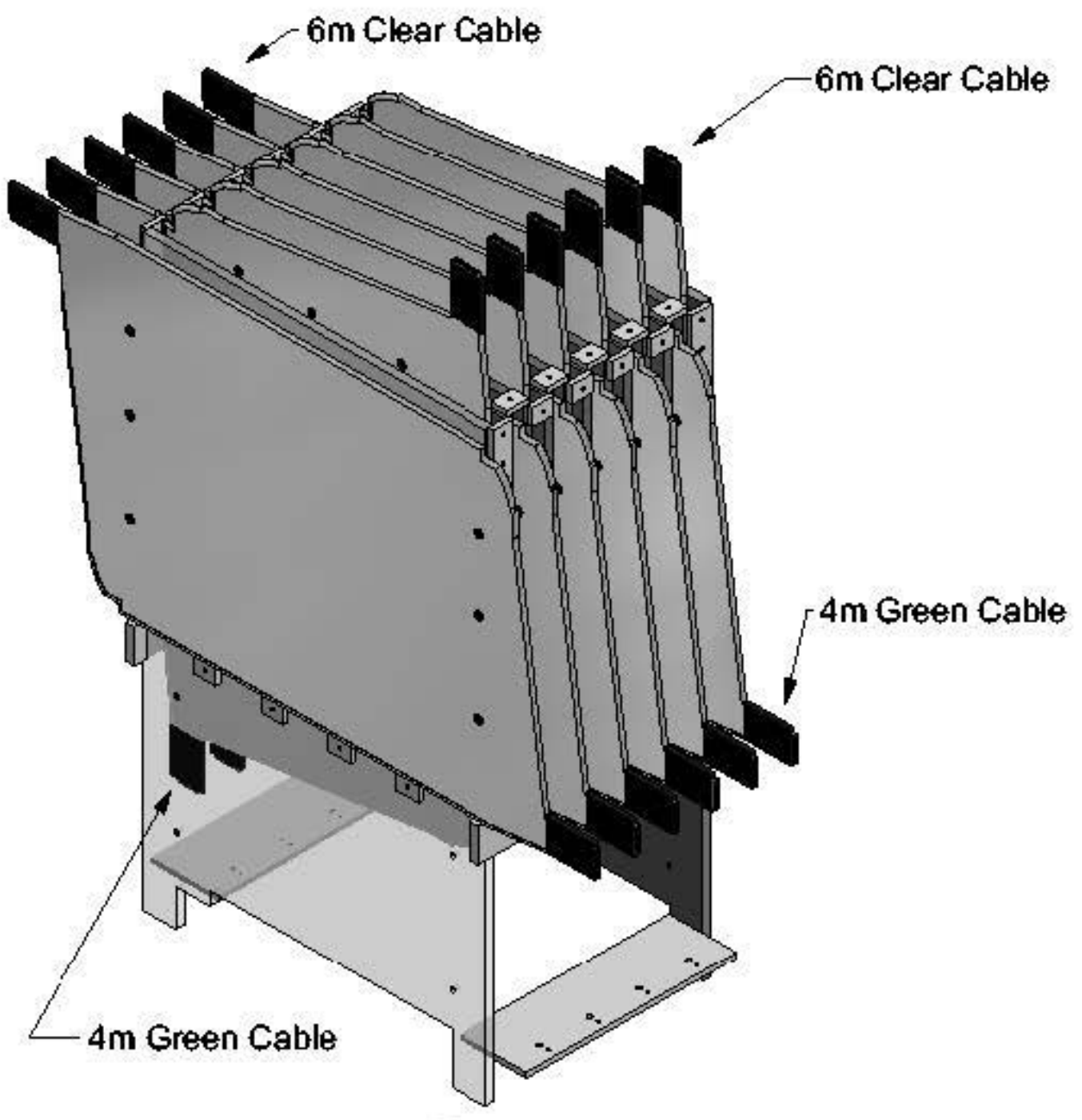

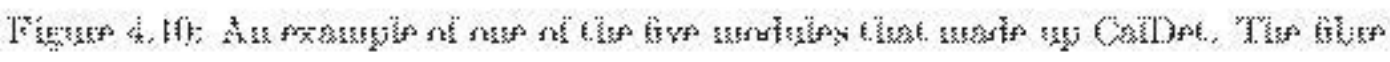

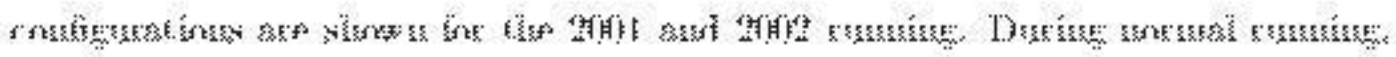

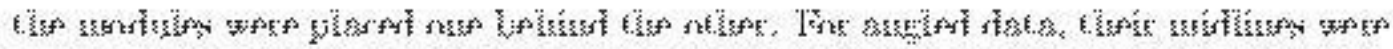

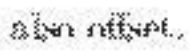




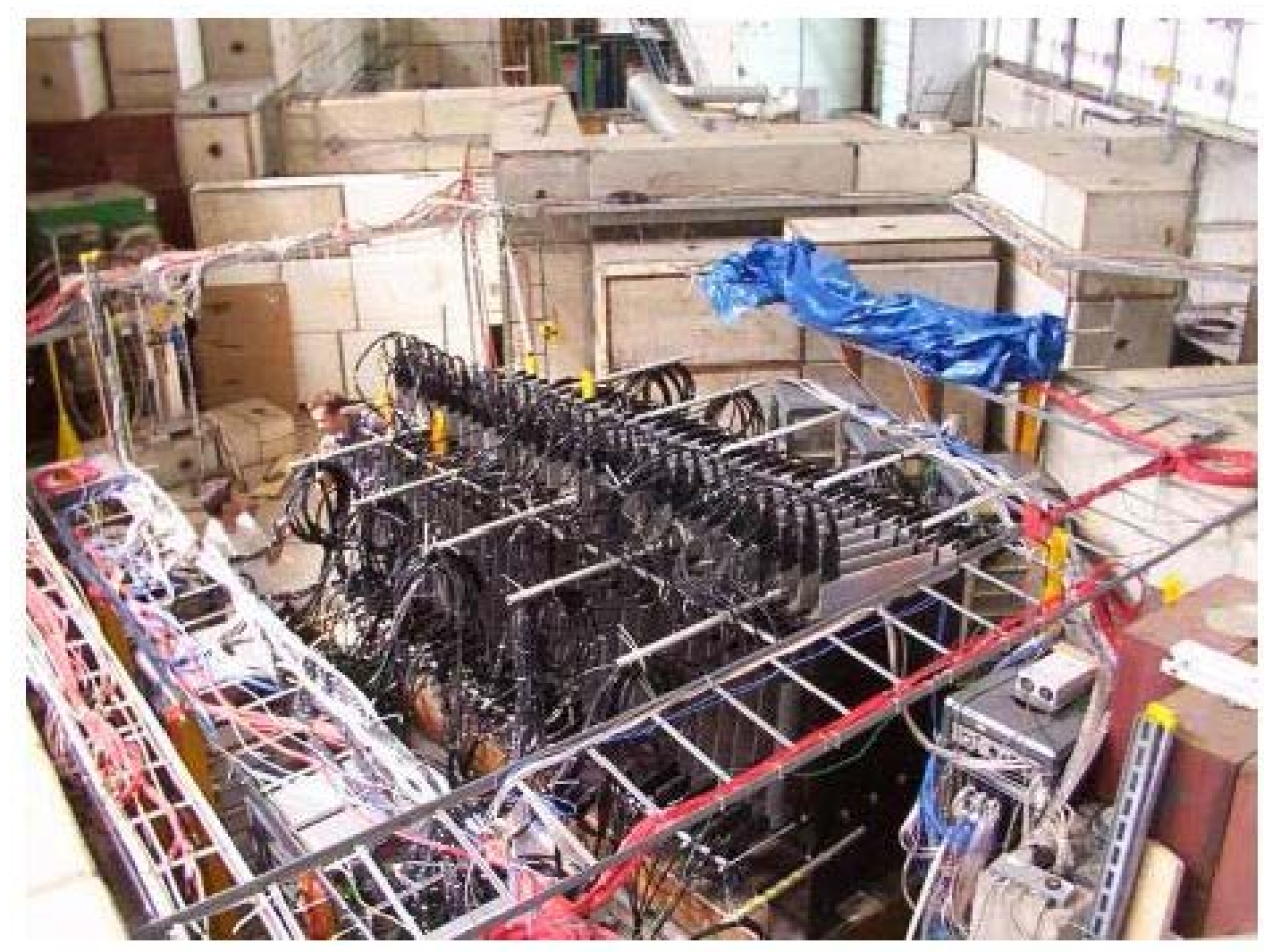

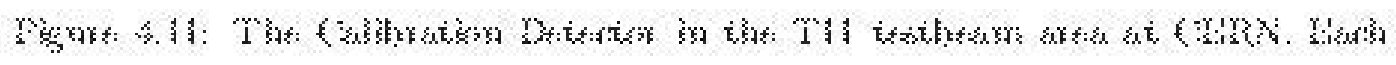

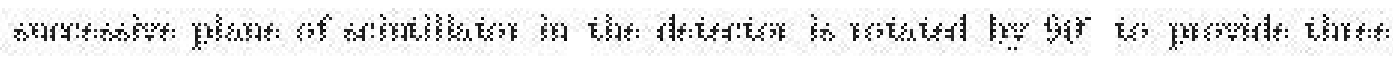
A

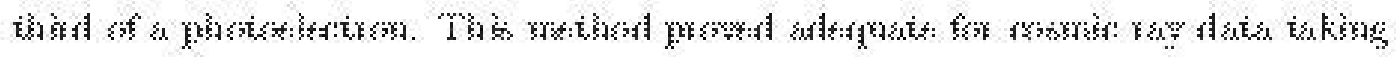

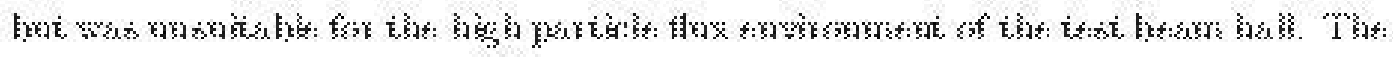

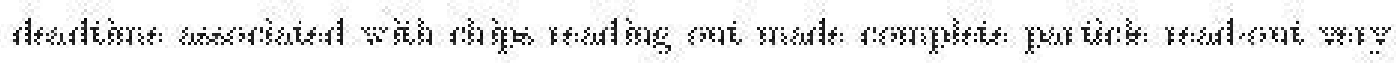

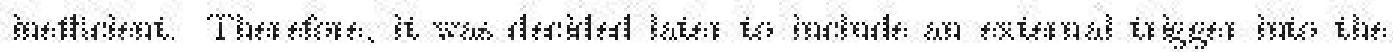

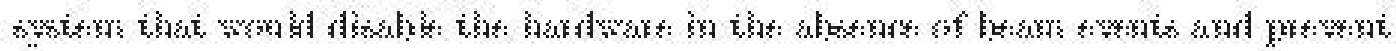
9t:

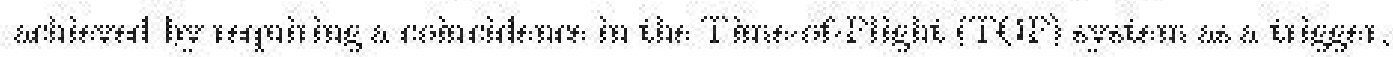

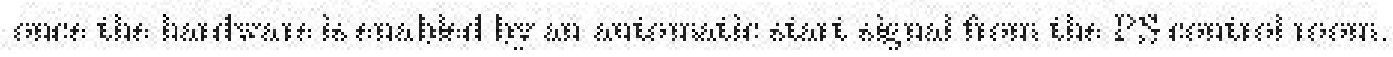

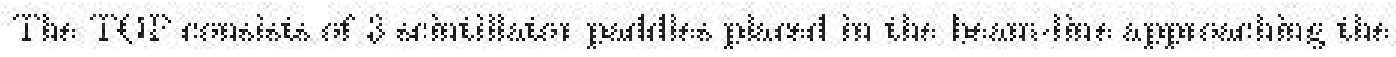

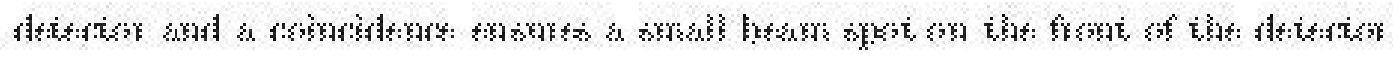

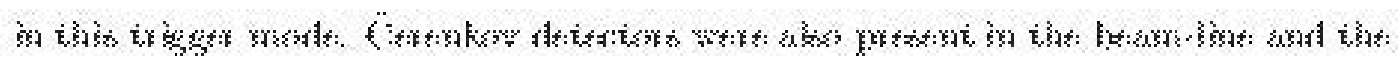




\begin{tabular}{|c|c|c|c|c|c|}
\hline \multirow[b]{2}{*}{ Year } & \multirow[b]{2}{*}{ Beamline } & \multirow[b]{2}{*}{ Configuration } & \multicolumn{2}{|c|}{ Fibre Length } & \multirow[b]{2}{*}{ Angle } \\
\hline & & & Clear & Green & \\
\hline 2001 & $\mathrm{~T} 11$ & Far, No Ext. Trig. & $6 \mathrm{~m}$ & $4 m$ & $0^{\circ}$ \\
\hline 2002 & $\mathrm{~T} 7$ & Far, No Ext. Trig. & $6 \mathrm{~m}$ & $4 m$ & $0^{\circ}$ \\
\hline 2002 & $\mathrm{~T} 11$ & Far/Near(7planes) & $6 \mathrm{~m}$ & $4 \mathrm{~m}$ & $0^{\circ}$ \\
\hline 2002 & $\mathrm{~T} 11$ & Far & $6 \mathrm{~m}$ & $4 \mathrm{~m}$ & $0^{\circ}$ \\
\hline 2002 & $\mathrm{~T} 11$ & Far & $6 \mathrm{~m}$ & $4 \mathrm{~m}$ & $30^{\circ}$ \\
\hline 2002 & $\mathrm{~T} 7$ & Far & $6 \mathrm{~m}$ & $4 \mathrm{~m}$ & $0^{\circ}$ \\
\hline 2003 & $\mathrm{~T} 7$ & Far/Near & $6 \mathrm{~m}$ & $3 m$ & $0^{\circ}$ \\
\hline 2003 & $\mathrm{~T} 7$ & Near & $6 \mathrm{~m}$ & $3 m+$ reflector & $0^{\circ}$ \\
\hline 2003 & $\mathrm{~T} 7$ & Near & $6 \mathrm{~m}$ & $1 \mathrm{~m}+$ reflector & $0^{\circ}$ \\
\hline 2003 & $\mathrm{~T} 11$ & Near & $6 \mathrm{~m}$ & $1 \mathrm{~m}+$ reflector & $0^{\circ}$ \\
\hline 2003 & $\mathrm{~T} 11$ & Near & $6 \mathrm{~m}$ & $1 \mathrm{~m}+$ reflector & $45^{\circ}$ \\
\hline 2003 & $\mathrm{~T} 11$ & Near & $6 \mathrm{~m}$ & $1 m+$ reflector & $30^{\circ}$ \\
\hline 2003 & $\mathrm{~T} 11$ & Near & $6 \mathrm{~m}$ & $1 \mathrm{~m}+$ reflector & $15^{\circ}$ \\
\hline
\end{tabular}

Table 4.2: The various configurations of CalDet that were used for data taking over the course of the test-beam runs at CalDet. $\mathrm{T} 7$ and $\mathrm{T} 11$ were respectively the higher and lower energy beamlines. Far refers to VA type read-out electronics and Near indicates that QIE electronics were used. No Ext. Trig. means that the signal from the PS was not used to enable the detector. 'Reflector' refers to the configuration where the read-out is QIE and at one end only; a reflective connector at the other end returns the signal to the read-out end. The angle indicates the angle of the beam with respect to the axis of the detector.

requirement of hits in these could be added as part of the external trigger. The external trigger maximises the number of true beam events that are recorded and also ensures accurate particle identification information for those events.

Once the hardware has been enabled and the VA chips have digitized a signal, they are read-out by the VA Read-out Controller or VARC. The VARC timestamps all channels of a particular chip with the time of the dynode trigger and also performs pedestal subtraction, common mode noise correction and zero suppression 
(sparsification) which significantly reduces the amount of data produced. The information is then passed to the DAQ which can perform some triggering and event reconstruction. Blocks of data are then written to disk which typically contain just one event and are known as 'Snarls'.

\subsection{CERN Test Beams}

CalDet was used in the East Hall test beam complex at CERN. The beams there are produced by directing protons from the PS (proton-synchrotron) accelerator onto one of many available targets. Electromagnets are then used to select all the particles in the resulting shower, of a definable momentum. CalDet was used in the $\mathrm{T} 7$ beam which had an available energy range of $0.5-10 \mathrm{GeV}$ and $\mathrm{T} 11$ which had a range of 0.5-3.5 GeV. Whilst not in the beam, CalDet sat in a wooden enclosure in the hall taking cosmic ray data. Apart from cosmic rays, CalDet was also exposed to an artificial, high energy source of muons which have been dubbed 'PS' muons. PS muons could be seen anywhere in the East Hall and seem to emanate from the PS ring (when in operation). In fact they provided an alternative strip-to-strip calibration method to cosmic rays.

A typical high momentum beam consists of protons, positrons, kaons and pions, some of which decay to muons. The polarity of the electromagnets can be switched to select out positively or negatively charged particles. The relative fraction of these particles in the beam depends on the charge and momentum selected.

Two systems were available to aid particle identification: the TOF and the Cerenkov detectors. The TOF is principally used to separate pions and protons since a proton of a given momentum will take longer to travel between two TOF paddles than a less massive pion. The cerenkov counters are aluminium tubes between 2.5 and $4.4 \mathrm{~m}$ long with a diameter of $15 \mathrm{~cm}$, that could be filled with $\mathrm{CO}_{2}$ to variable pressures. At low pressures, only very fast moving particles will trigger the cerenkov detector, allowing particles with certain velocities to be selected by tuning the pressure. Given that all of the particles in any beam had the same momentum, the counters could be set to fire on electrons only, electrons and muons or electrons, muons and pions. 


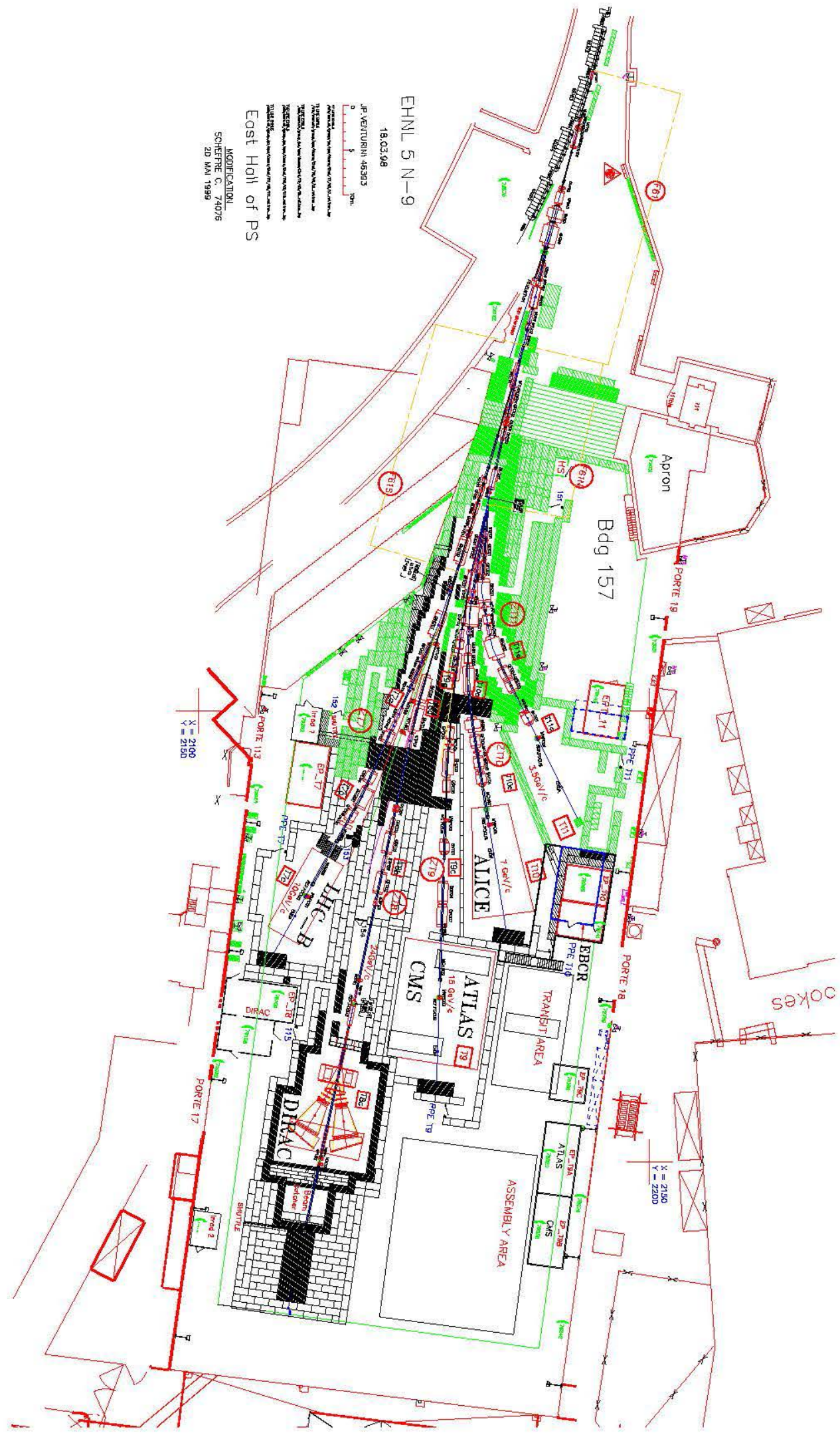

Figure 4.12: The East Hall at CERN. CalDet took data in the T11 and T7 testbeams. 


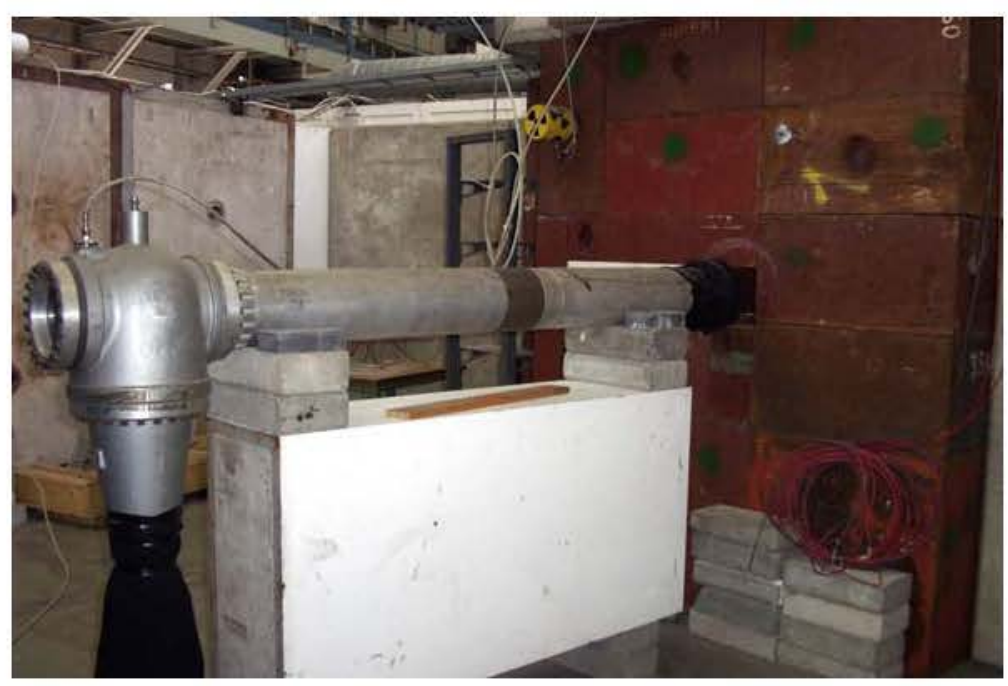

Figme 4.13: A photo af a Cerenkov detector at CalDet. Particles travel down the length of the $\mathrm{CO}_{2}$ filled almminm pipe (right to left) creating cerenkov radiation. This is reflected by a mimror to a PMT (bottom left) where it is detected.

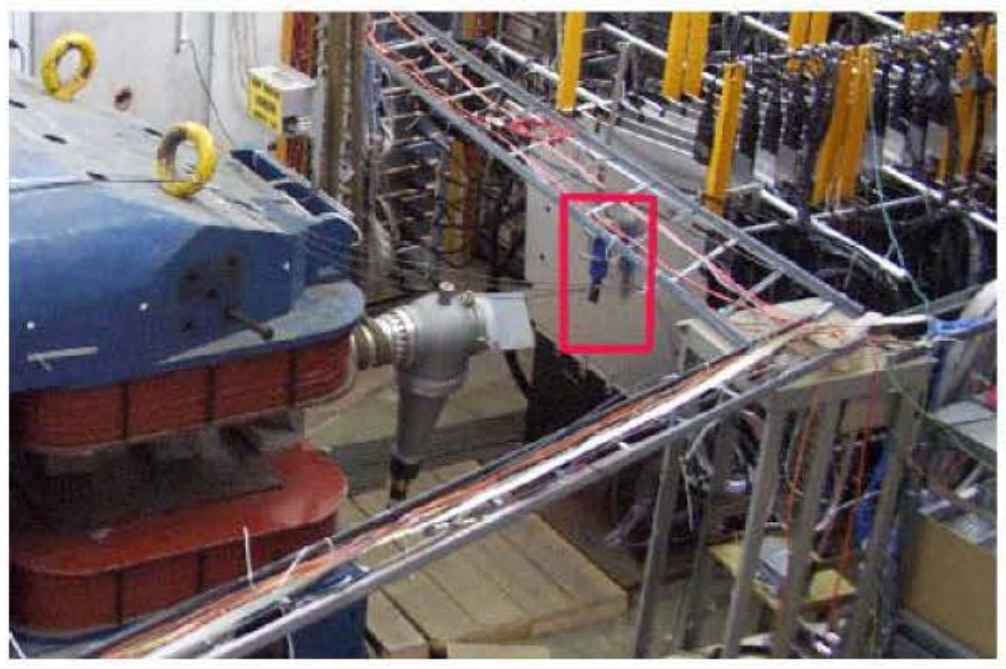

Figne in.14: A phote of CalDet in the T7 beamine in 2003. The ent of a Cerenkov detecter can be seen protruding from a large electromagnet (mused) on the left. Ome of the TOF padkles shown in the red box. right in front of CalDet. Recuming a comcidence in all the TOF paddes ensures a small beam-spot of particles that have come directly down the beam pipe. 


\subsection{MINOS Physics}

The principle goal of the MINOS experiment is to conclusively establish the cause of $\nu_{t b}$ disappearance and in the case that it is neutrino oscillations, to measure the oscillation parameters to $10 \%$. The main way this is achieved is by measuring and comparing the NC (neutral current) and CC (charged current) spectra at the Near and Far detectors. A reduction in the expected number of $\mathrm{CC} \nu_{\mu}$ events in the Far detector is an indication of oscillations and the relative spectra yield information about $\sin ^{2} 2 \theta$ and $\Delta \mathrm{m}^{2}$.

Events in the MINOS detectors can be simplified into two types: 'Long' and 'Short'. Long events arise mainly from $\mathrm{CC} \nu$ interactions which produce a hadronic shower and a distinctive muon track in the detector. A fraction of $\mathrm{CC} \nu_{T}$ interactions will also appear the same if the tau happens to decay to a muon. Short events will occur from any neutrino species as a result of NC scattering. A small number of background $\nu_{e}$ and most $\nu_{T}$ CC events will also be similar.

The T-test 'ratio of ratios' can then be constructed as a function of energy:

$$
\frac{(N(\text { short }) / N(\text { long }))_{\text {Near }}}{(N(\text { short }) / N(\text { long }))_{\text {Far }}}
$$

This statistic is not affected by the relative fluxes at the two detectors and is sensitive to both depletion of $\nu_{p}$ events at the far detector and a corresponding increase of $\nu_{e}$ and $\nu_{\tau}$ events in the case of neutrino oscillations. If no increase is seen, this could be an indication of $\nu_{\mu} \rightarrow \nu_{\text {sterile. }}$. Theory predicts that the number of long events will exceed the number of short events at both detectors but the NC cross-section rises at lower energies; this means that the more accurate the calibration is, the lower the minimum energy threshold can be set and the better the statistics that can be achieved. Figure 4.16 shows that for 2 years running, MINOS could be sensitive to values of $\Delta \mathrm{m}^{2}$ as low as $10^{-3} \mathrm{eV}^{2}$ for $\nu_{\mu} \rightarrow \nu_{\tau}$ oscillations with maximal mixing.

An alternative technique allows an even greater reach on $\Delta \mathrm{m}^{2}$. By extrapolating the Near spectrum to the Far Detector using Monte Carlo, an expected, unoscillated spectrum can be produced. The ratio of this quantity to that observed in the data would give a 'dip' at a specific energy if oscillations are taking place (See Figure 4.17). The depth of this dip gives information on $\sin ^{2} 2 \theta$ and the exact 


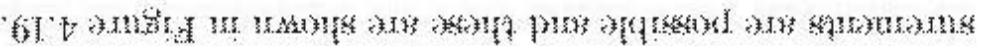

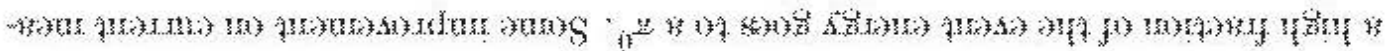

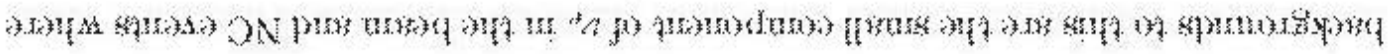
.

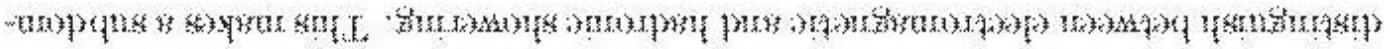

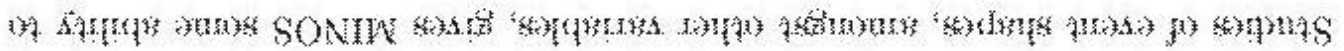

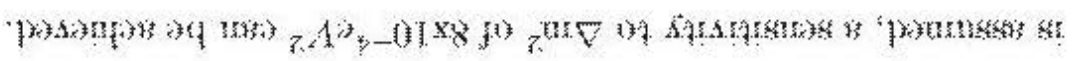

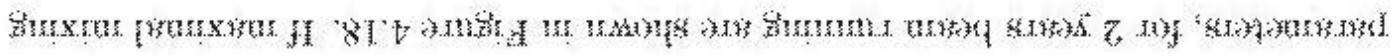

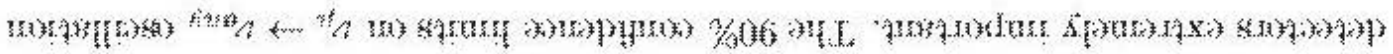

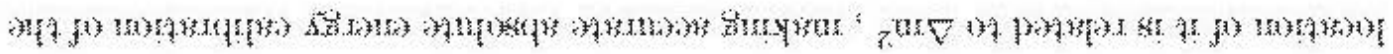

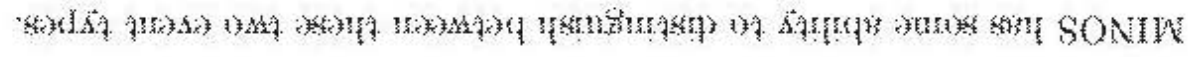

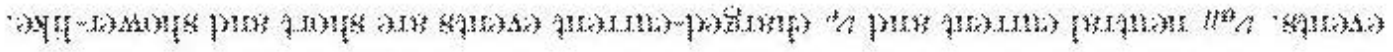

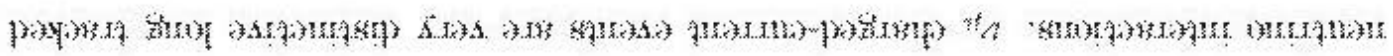

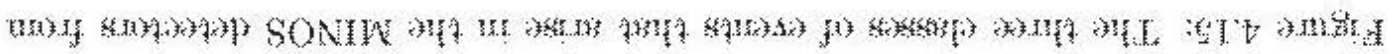
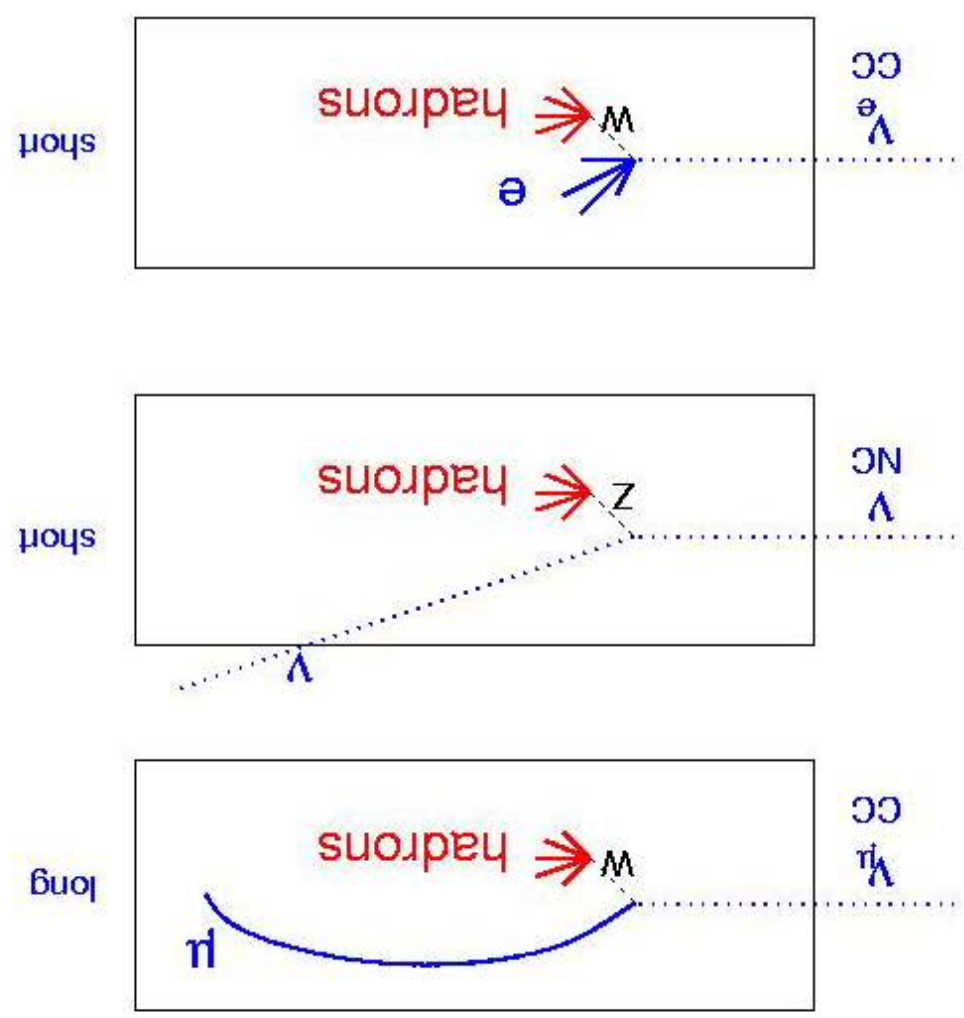


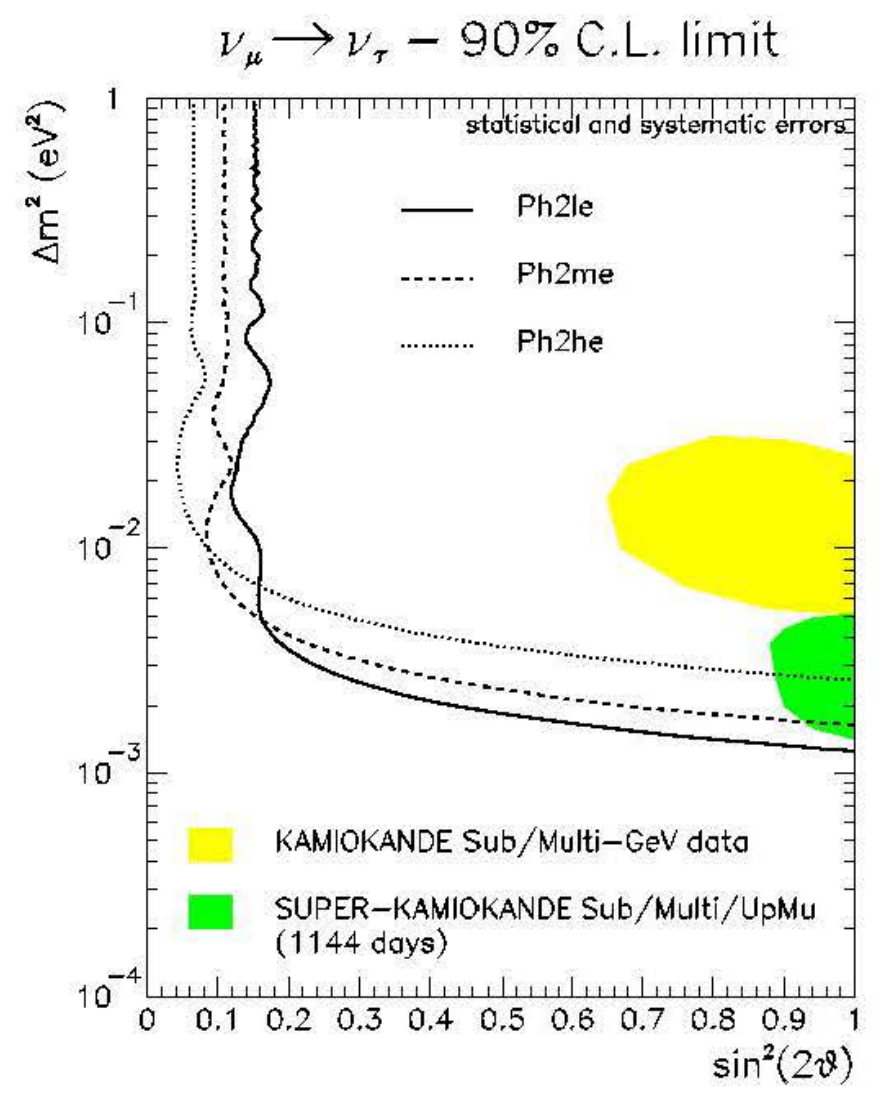

Figure 4.16: The $90 \%$ confidence limits on $z_{\mu} \rightarrow z_{r}$ ascillation parameters by applixation of the T-Test method. Limits are shown for the three separde bean configurations, assuning that after two years of ruming no evidence for oscillation is seen. The Kaniokande and Super-K prefered regions are also shown. 

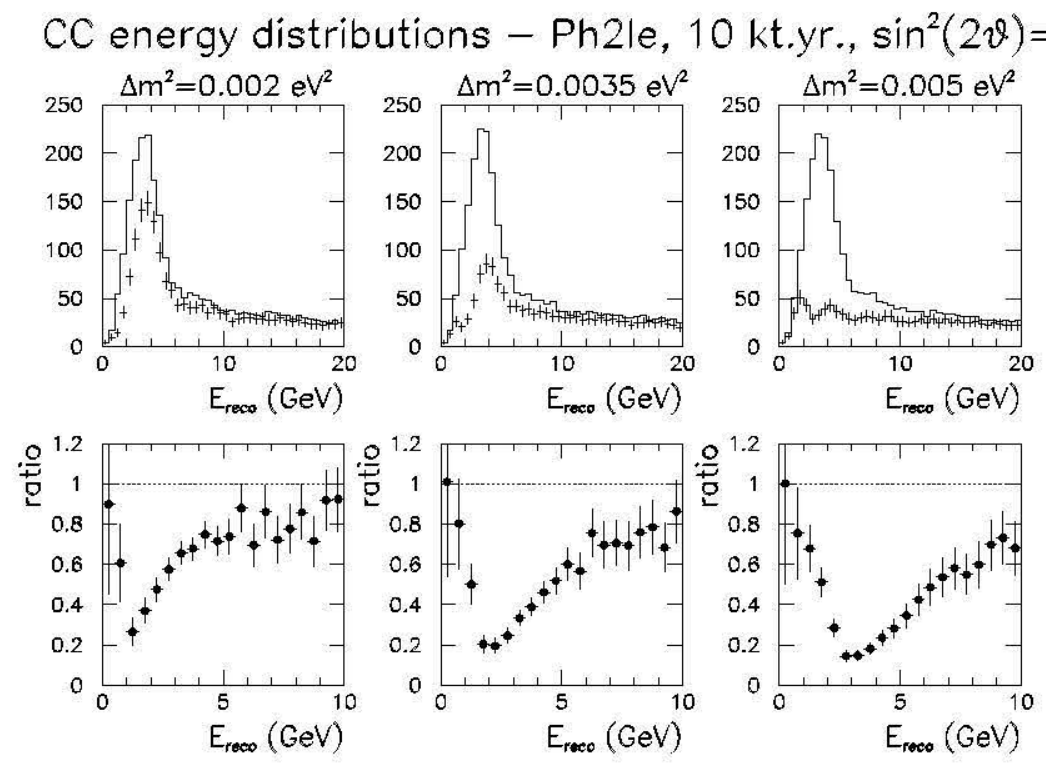

Figure 4.17: On the top are the oscillated and unoscillated energy spectra for charged current $\nu_{\mu}$ events, for three values of $\Delta \mathrm{m}^{2}$. On the bottom is the ratio of these two spectra. The depth of the dip in the ratio is determined by $\sin ^{2} 2 \theta$, the position of the dip by $\Delta \mathrm{m}^{2}$.

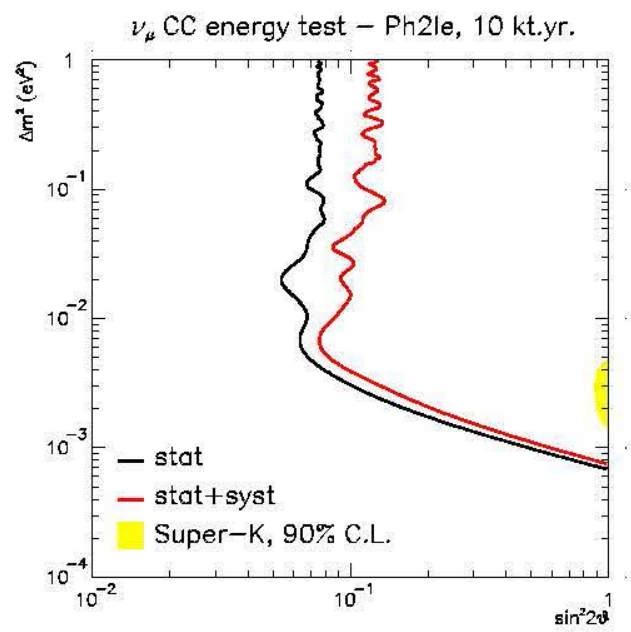

Figure 4.18: Limit plots showing the $90 \%$ confidence limits, from two years of running, assuming no oscillation signal is seen in the charged current $\nu_{p}$ energy spectrum. Beam systematics are included in this plot. 


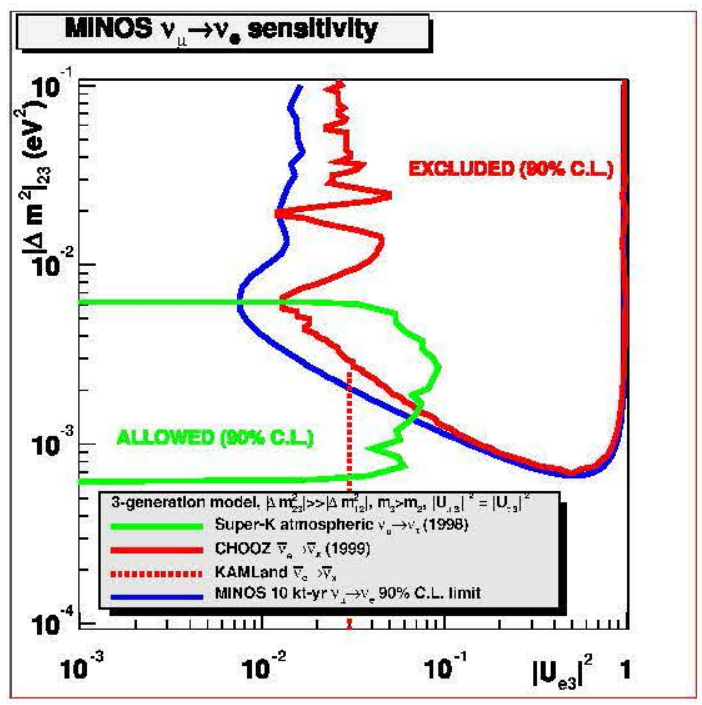

Figure 4.19: Limit plots showing the $90 \%$ confidence limits, from two years of running, assuming no oscillations, from $\nu_{e}$ appearance. 


\section{Chapter 5}

\section{Calibration and Crosstalk}

In order to make sense of MINOS data, it is important to have established an accurate chain of calibration. Without calibration, signals cannot be compared liketo-like between different parts of a detector, or detector to detector. This requires that the response of each part of the detectors from the scintillator to the output of the digital electronics be understood thoroughly. There are many other higher level detector, electronics and envirommental effects that also influence the interpretation of the data. One such example, seen in all the MINOS detectors, is PMT Crosstalk, which arises from the close proximity of the PMT pixels to one another, causing false signals.

This chapter discusses the objectives and methodology of the calibration and describes the causes and effects of PMT crosstalk. An algorithm to remove PMT crosstalk from CalDet data is presented and tested.

\subsection{The Calibration Chain}

When a particle is seen in the MINOS detectors, a large number of steps need to be taken before a reliable measurement of the type and energy of that particle can be reported. It is the job of the calibration to account for effects in the detector elements such as:

- Scintillator: Particle type/energy, Fluor quantity, Path length 
- Fibres: Light transmission to fibres, Wavelength shifting fibre conversion efficiency, attenuation, losses at optical couplings

- PMTs: PMT glass transmission, quantum and collection efficiencies, pixel to pixel gain differences, non-linearity

\section{- Electronics: channel to channel digitization differences, QIE/VA}

The MINOS electronics are calibrated by a process known as charge injection. Known quantities of charge are digitized and the response of the electronics can be established. Far detector-like VA electronics have a single range ADC for which the linearity can be checked with a few measurements. The Near detector-like multiranging $\mathrm{ADCs}$ require many more measurements. The electronics also continually digitizes channels that are not being hit in order to construct a noise pedestal. The pedestal is subtracted from the data online to ensure only real hits are written.

It is important to ensure that we can calibrate the optical read-out with known quantities of light. For this, the light injection system (LI) has been developed. The LI uses 'Pulser Boxes' which house ultra-voilet LEDs and are set up to deliver light along optical fibres to every PMT pixel and to several PIN diodes. The PIN diode has been shown to be very linear [72] and is read out along the same electronic chain, allowing a measurement of the PMT response to be made. This can be done for many light intensities to generate a linearity curve, which is expected to flatten out at higher intensities due to space-charge effects in the PMT. The process of measuring the PMTs response over a large range is slow so it is only undertaken roughly once a month. On much shorter time scales, a single 'drift' point is measured and the curve shifted up or down slightly to fit to it.

Within the detectors, we expect the specifications of invidual scintillator strips to vary slightly from one to another. To normalise them, they all need to be exposed to some common source. MINOS employs cosmic ray muons that pass all the way through the detectors to do this (See Figure 5.2), since they are minimum ionising particles and deposit approximately the same amount of energy in every strip they traverse. The spectrum that each detector sees is different however so the samples are not directly comparable but sufficient exposure for adequate strip-to-strip calibration 


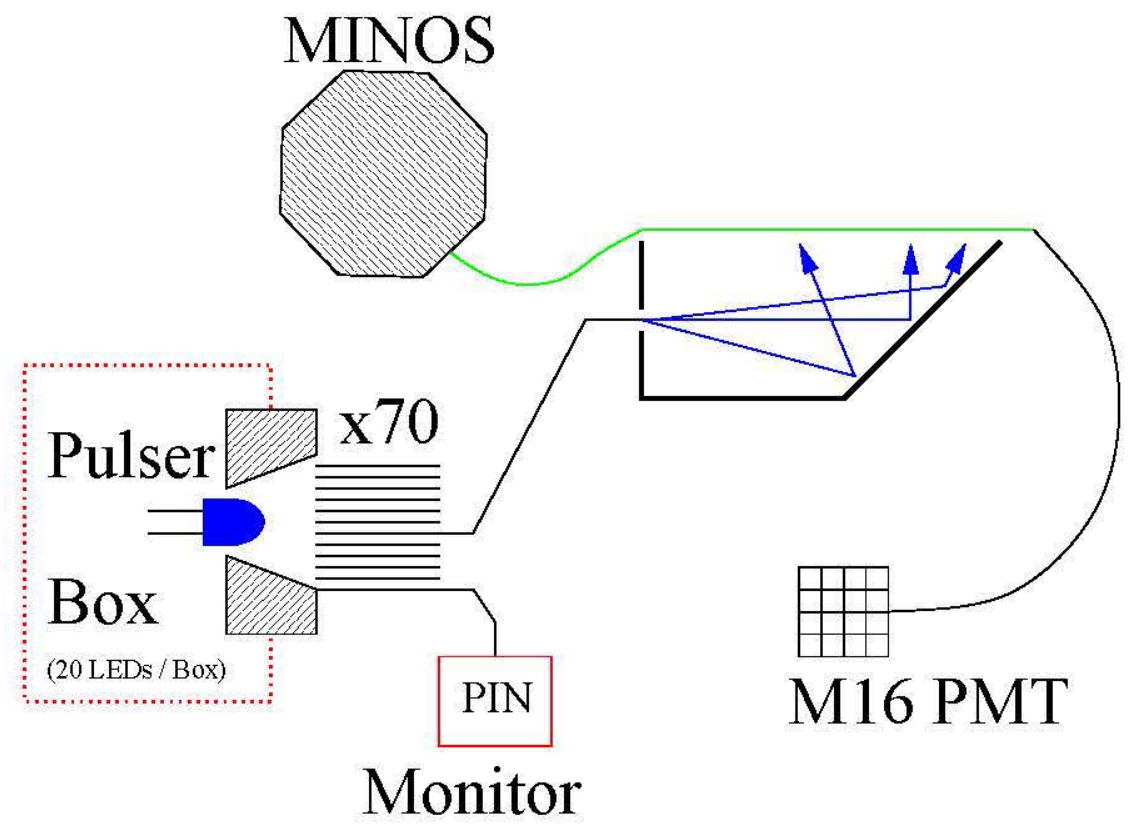

Figure 5.1: A schematic diagram of the MINOS light injection system. UV LEDs in the pulser boxes illuminate many optical fibres, one of which goes to a PIN diode as a reference. The other fibres go to 'ashtrays' where the light is delivered to the WLS fibres in the MINOS detectors and hence to a PMT per end. This diagram illustrates the LI system in the Far Detector. CalDet has the same system but on a smaller scale and the Near Detector has single-ended read-out and M64 PMTs.

(to $2.4 \%$ in the Far Detector and $0.3 \%$ in the Near Detector) can be achieved within a month. At CalDet, PS muons can also be used for calibration purposes (see Section 5.1.1).

In order to compare data in the detectors meaningfully, an equivalent set of particles must be found in all detectors. Stopping muons are used since their energy can be determined by their range in the detectors, or by their curvature if a magnetic field is present. For the Near and Far Detectors, the stopping muons are cosmic in origin, at CalDet test beam muons are used since the detector is too small to collect a sizeable sample of cosmic ray muons that have unambiguously stopped. Figure 5.3 shows an example of a stopping beam muon at CalDet. Measurements of the characteristics of the energy lost by these particles as they cross the detectors $(\mathrm{dE} / \mathrm{dX})$ can be used to ensure that particles are being compared like-to-like. An 
example of a type of particle that can be misidentified are so called 'punch- or sailthrough' pions that occasionally behave similarly to muons in the detectors. With accurate relative calibration of the detectors, event energies can be described in terms of Muon Energy Units (MEUs) which are a common unit to all three detectors. An MEU is defined as the response of a particular detector to a $1 \mathrm{GeV}$ muon travelling perpendicularly through 1 plane of scintillator ${ }^{1}$. An MEU scale which is consistent to $2 \%$ across the detectors is one of the goals of MINOS.

The final stage of calibration is to be able to convert from MEUs to visible energy. This was one of the main functions of CalDet since it could be exposed to several particle species of known momenta. This allowed event topologies and hadronic and electromagnetic energy deposition to be compared. A target of $5 \%$ precision on the absolute energy resolution of the detectors has been set. Together with simulations, a high degree of accuracy on the energy of incident neutrinos from their interactions in the detectors, is achievable.

\subsubsection{PS muons}

At CalDet, as well as cosmic and beam muons, a third class of these particles are also observed. Dubbed 'PS muons', they are believed to be muons artificially created in the PS ring at CERN that subsequently escape. In the T11 test beam, PS muons tend to enter the detector at roughly the same height as beam muons, and stay at that height for their entire passage through. However, rather than coming down the beam-pipe and arriving at the detector in the centre-front of CaIDet, PS muons enter at some point off the beam spot, or even through the side of the detector (Figure 5.4). It could be argued that these muons are cosmic ray muons whose source is the horizon. However the observed flux is far too great to be explained in this way. PS muons always appear to be through-going, implying that their energy is at least $2.3 \mathrm{GeV}$ and more likely, much higher. In T7, a similar phenemenon is observed. These PS muons do enter the detector down the beam-pipe as expected of normal beam muons but they are through-going, regardless of the setting of the

\footnotetext{
'The term 'MIP' is sometimes also used, but fell out of favour on account of it being deemed misleading
} 
beam momentum selecting magnets. By extrapolating back the source directions of the PS muons in T7 and T11 it was possible to determine that the likely point of production of these particles was the septum magnet where the primary beam is extracted from the PS accelerator.

In $\mathrm{T} 11$, it is possible to perform a strip-to-strip calibration of the detector using PS muons, although the coverage is not homogeneous, making the traditional cosmic ray calibration preferable. It is not possible to calibrate with $\mathrm{PS}$ muons in $\mathrm{T} 7$.

\subsection{Crosstalk}

\subsubsection{Introduction}

Crosstalk is a well known PMT phenomenon - it has been studied in detail by various institutions in test-stand setups [79] [80] [81] [82] [83] [86] [87] [88]. It arises in both the M16 and M64 PMTs that are used in the MINOS detectors. In the case of M16s, the PMT face has a $4 \times 4$ array of photomultiplier pixels on it. Crosstalk occurs when a part of the digitized charge from one pixel is read-out on another. This can happen when photons are scattered from their desired course at the optical interface or the glass of the PMT, or photoelectrons skip across to a neighbouring dynode chain; this is known as optical crosstalk and is the most dangerous form. Charge can also leak from pixel to pixel if electrons spill into an adjacent dynode chain; this is known as electrical crosstalk. Test stands see a large amount of crosstalk of this form but it is generally less serious than optical crosstalk as only the high energy tail of the distribution exceeds the nominal read-out. threshold of 0.3 photoelectrons.

The effect of crosstalk on the data is to produce the appearance of false 'hits' on scintillator strips in the detector. The pattern of PMT pixels to strips was designed to minimise the effect of this by having no adjacent strips connected to adjacent pixels. As such, crosstalk hits are often seen 'in the wings' of the beam data; for example, a typical muon would traverse the detector hitting, say, strip 11 in each plane passed and any associated crosstalk hits would be seen in the regions mapped to strips 3-6 and 15-19. In this case, it is obvious where the real muon track is and 


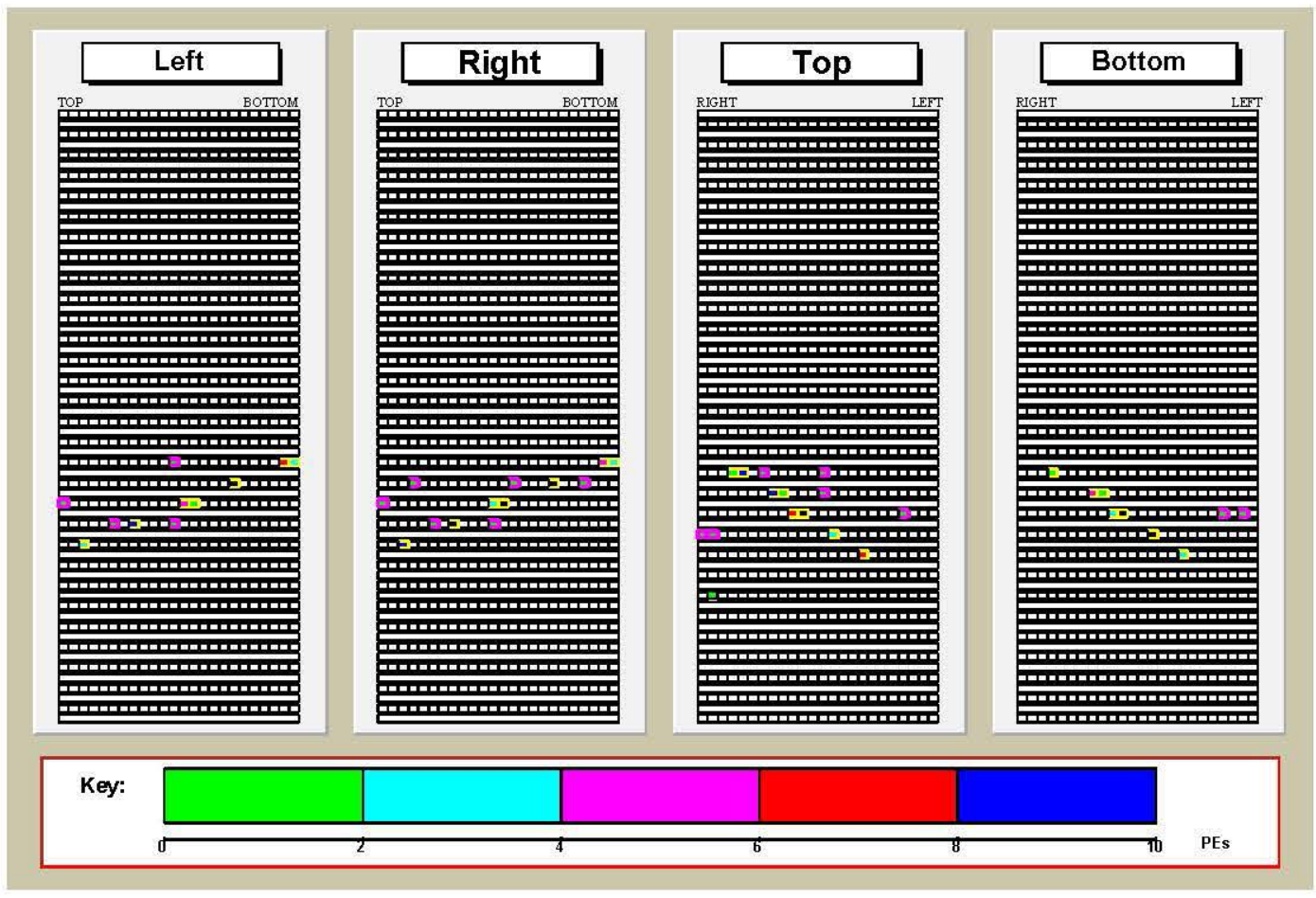

Figure 5.2: An example of a through-going cosmic ray muon at CalDet. The muon entered in the top-left of the detector, exited from the bottom-right and was contained roughly within the third quarter of the detector from the front. Common characteristics of cosmic rays muons are entering from the top of the detector and having a short, highly angled path. Very few cosmic ray muons can be shown to have stopped unambiguously within the detector. Coloured squares indicate where scintillator strips have been 'hit'. Yellow bordered squares indicate hits deemed to be part of a track. Purple bordered hits are prospective hits caused by crosstalk. The colour of the square relates to the scale at the bottom of the plot and shows the energy deposition in a particular strip, measured in number of photoelectrons. 


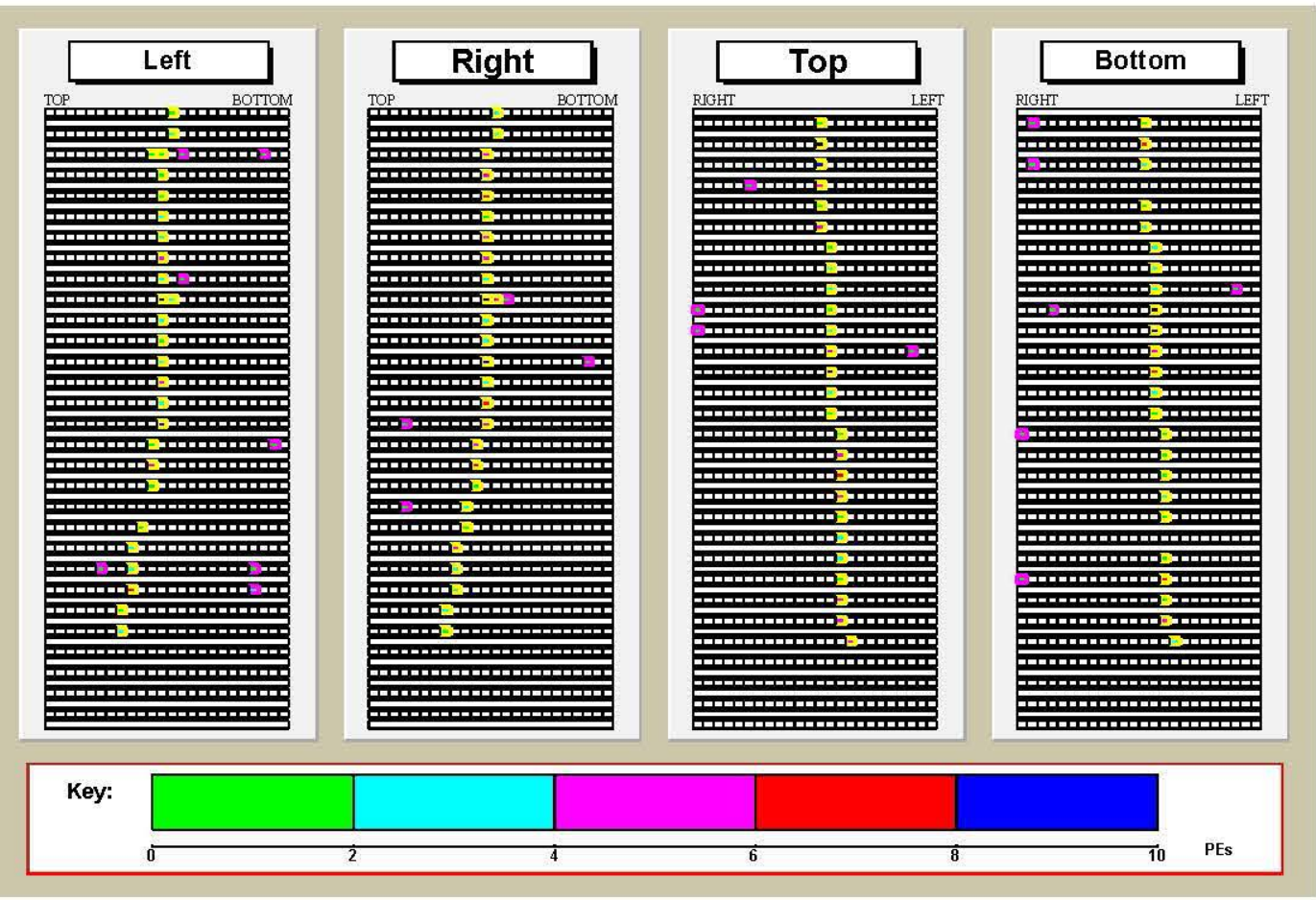

Figure 5.3: An example of a $1.8 \mathrm{GeV}$ beam muon, as produced in the CERN test beams. The muon has entered the front of the detector (top of the page) in the centre (in the middle of each view) and has remained roughly in the centre the entire way through. It appears as though the muon has run out of energy and stopped in the last quarter of the detector; it is possible although unlikely that the muon travelled all the way through the detector with the final hits not being seen for some reason, such as a read-out hole or chips suffering from dead-time. Coloured squares indicate where scintillator strips have been 'hit'. Yellow bordered squares indicate hits deemed to be part of a track. Purple bordered hits are prospective hits caused by crosstalk. The colour of the square relates to the scale at the bottom of the plot and shows the energy deposition in a particular strip, measured in number of photoelectrons. 


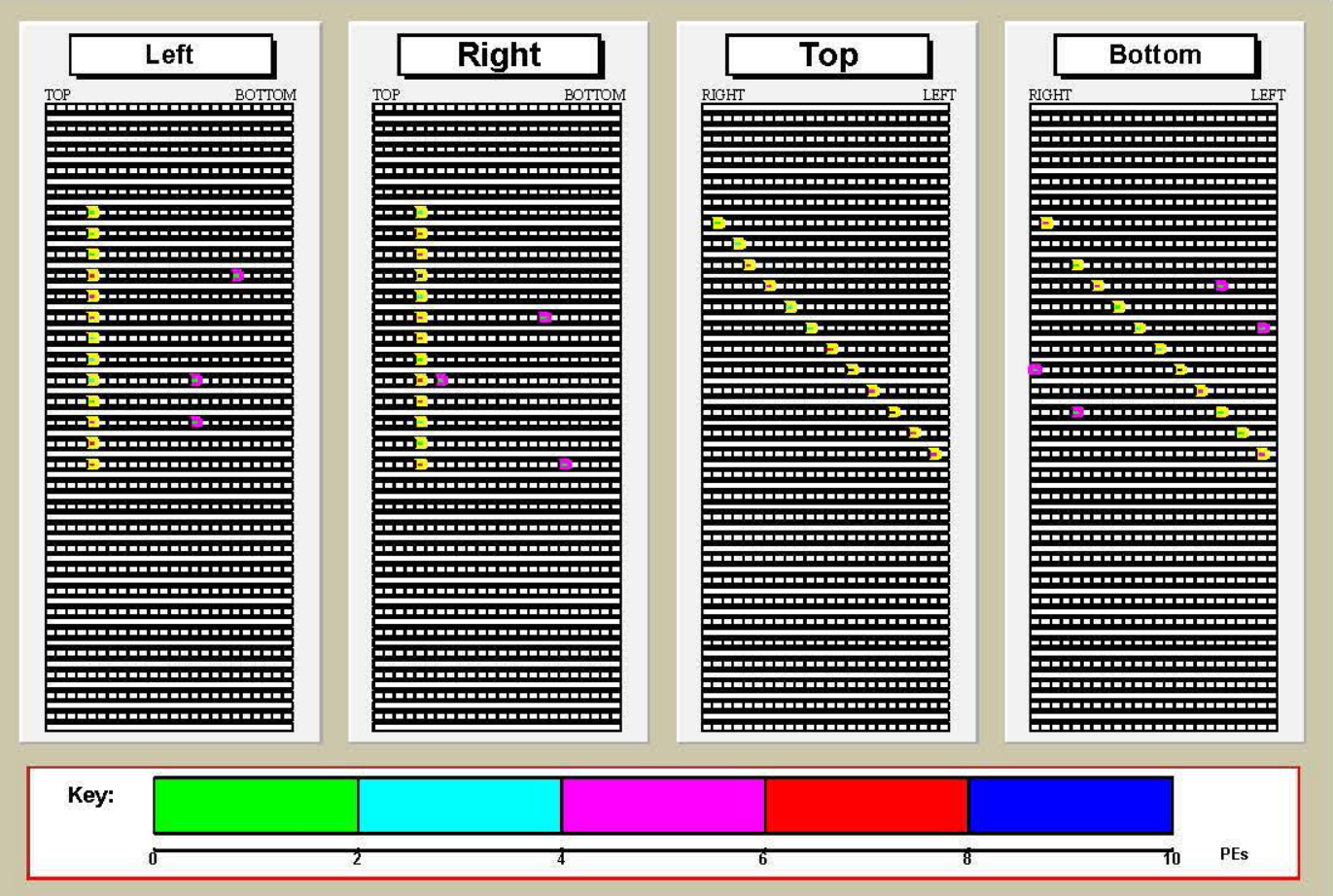

Figure 5.4: An example of a PS muon, thought to arise from losses in the PS ring at CERN. Events of this type tend to travel through the detector at a constant height (like beam muons) but from one side to the other (like cosmic muons). Coloured squares indicate where scintillator strips have been 'hit'. Yellow bordered squares indicate hits deemed to be part of a track. Purple bordered hits are prospective hits caused by crosstalk. The colour of the square relates to the scale at the bottom of the plot and shows the energy deposition in a particular strip, measured in number of photoelectrons.

what is crosstalk.

The situation becomes more complicated if the track curves. At CalDet, a 'swimmer' type muon tracking code is used for calibration and partly for particle identification [84]. If the code comes across a crosstalk hit, it can cause a miscalculation of the energy deposited in a given plane and, in a worst case scenario, cause the swimmer to lose the real track entirely, see Figure 5.8.

A further problem is that of inter-plane crosstalk. At CalDet, all of the strips of one end (side) of each pair of planes are connected to three PMTs in a mux box. 


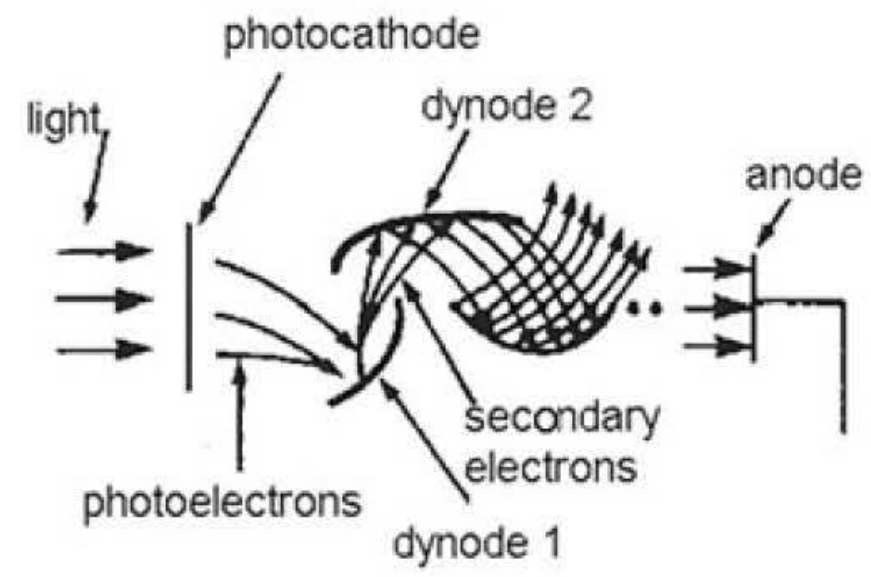

Figure 5.5: A diagram illustrating how PMTs operate. Light is incident on a photomcathode, creatimg photoelectrons. These are accelerated by an electric feld towards the first dymode where secombary electroms are produced. There are 12 dymodes: by which time the number of electrons has been multiplied by aromud $3 \times 10^{5}$ (the gane). The signal is then picked up on the anode. This diagram represents one PMT

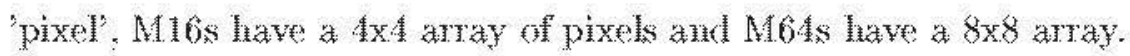

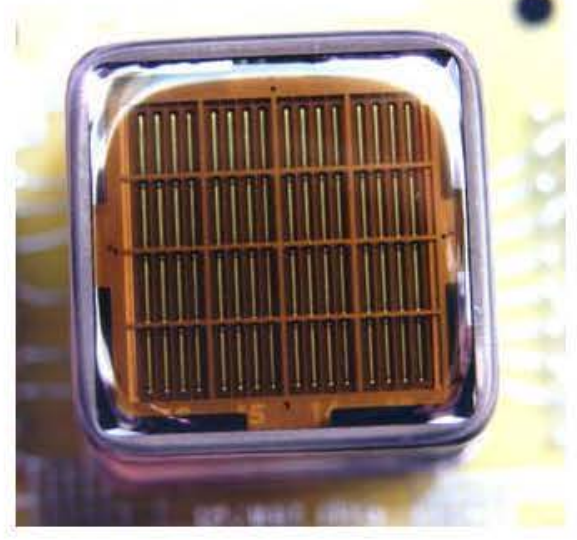

Figure 5.6: An M16 PMT. The etxin array of pixels and 'venetian blind' structure of the photocathores are visible.

$(24 \times 2$ strips $=3 \times 16$ pixels $)$ This mecessitates that 8 strips from each of the two separate planes are optically linked to the same PMT face. Shonld crosstalk occur. the false hits can appear in the other plane to that which it was generated in. This problem is accentuated by the fact that the pixel-strip pattern of the PMT plexing tends to place these crosstalk hits in line with the beam. It has beem shown that 
there can be cases where crosstalk hits fall into genuine 'gaps' in beam tracks; it is impossible to unambiguously identify these hits as being or not being crosstalk, but fortunately this only occurs rarely.

Cosmic muon data (which is used for the strip-to-strip calibration of CalDet [84]) is not even subject to the luxury of having its associated crosstalk 'in the wings' and away from the particle track since these particles tend to enter the sides and top of the detector, hitting fewer planes and more strips per plane than beam muons (See Figure 5.2).

A final consideration is the pixel-spot structure of the CalDet fibre-PMT interface. At the Far Detector, each M16 PMT pixel receives light from not one but eight scintillator strips in order to save on electronics (see Section 4.5, Figure 4.9). The eight strips, whilst on the same plane, are separated such that a track should never produce a signal in more than one of the eight fibres at a time. A different plexing regime on the other end ensures that the correct scintillator hit can always be uniquely identified. This procedure is known as 'de-multiplexing' and is performed by the offline reconstruction code. At CalDet, there is no need for more than one scintillator strip to go to a given PMT pixel, but the interface remains the same, with the redundant seven fibre openings being filled. The position of the active fibre on the pixel face is known as the spot. For every PMT at CalDet, a given pixel number always receives its light from a singular spot position as shown in Table 5.1.

This enables the effect of the spot position to be studied, the assumption being that less optical crosstalk would be produced from the more centrally lying spots 4 and 5.

It should be noted however that crosstalk is a random process and by simply requiring that a hit be seen by both ends of the read-out of a given plane, a large fraction of the crosstalk and other spurious hits such can be removed.

\subsubsection{Characteristics of Crosstalk in Data}

Figure 5.9 shows a plot of the crosstalk from pixel 1 seen in other pixels. The spectrum of the resultant crosstalk in pixel 6 has been enlarged to make the 1 p.e. 


\begin{tabular}{|c|c||c|c|}
\hline Pixel & Spot & Pixel & Spot \\
\hline 1 & 6 & 9 & 3 \\
2 & 3 & 10 & 2 \\
3 & 4 & 11 & 4 \\
4 & 5 & 12 & 2 \\
5 & 1 & 13 & 1 \\
6 & 8 & 14 & 6 \\
7 & 8 & 15 & 5 \\
8 & 7 & 16 & 7 \\
\hline
\end{tabular}

Table 5.1: Pixel to spot comfiguratom at CaIDet.

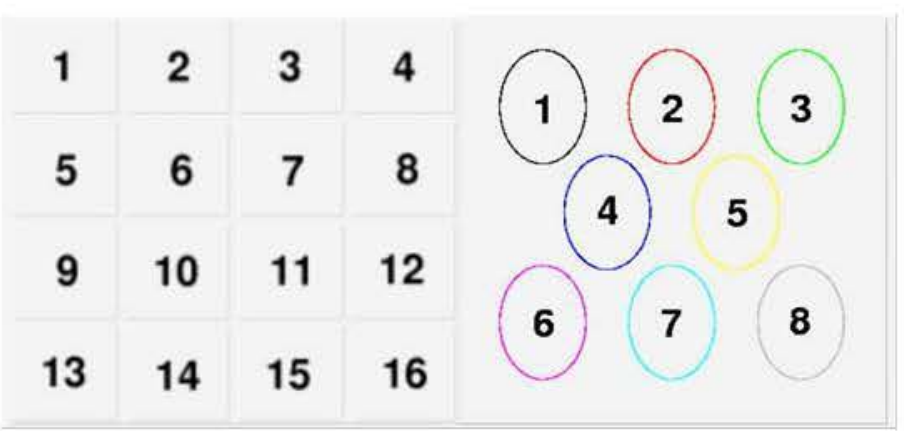

Figme 5.7: Left: Pixel mmbermgen PMT faces. Note: This is the comventim nsed in this docment. Pixel mmbermg in the MINOS Software Plex goes from 0 to 15. Right: Spot positions. Each pixel has a spot configuration of this form.

peak more visible; it can easily be identifed as the secom peak at about 80 ADC comits. For simplification, crosstalk in and greater than the 1 p.e. peak is defoned as optical chosstalk and that below as electrical. This defuntion is clearly mot perfect (especially in the region aromin 50 ADCs) but for most purposes it is not necessary to be able to say what variety of crosstalk a given hit is.

For the most part, crosstalk only spreats from a given pixel to its nearest enght neighbours. This is not always true; in Figme 5.9 crosstalking to a pixel two away from the somed hit is sem at the 0.01\% level and certainly it has been observed in test-stand data. The crosstalk algonithm that has been developed does not attempt to identify crosstalk hits of this kind due to the marked increase mu processing that 

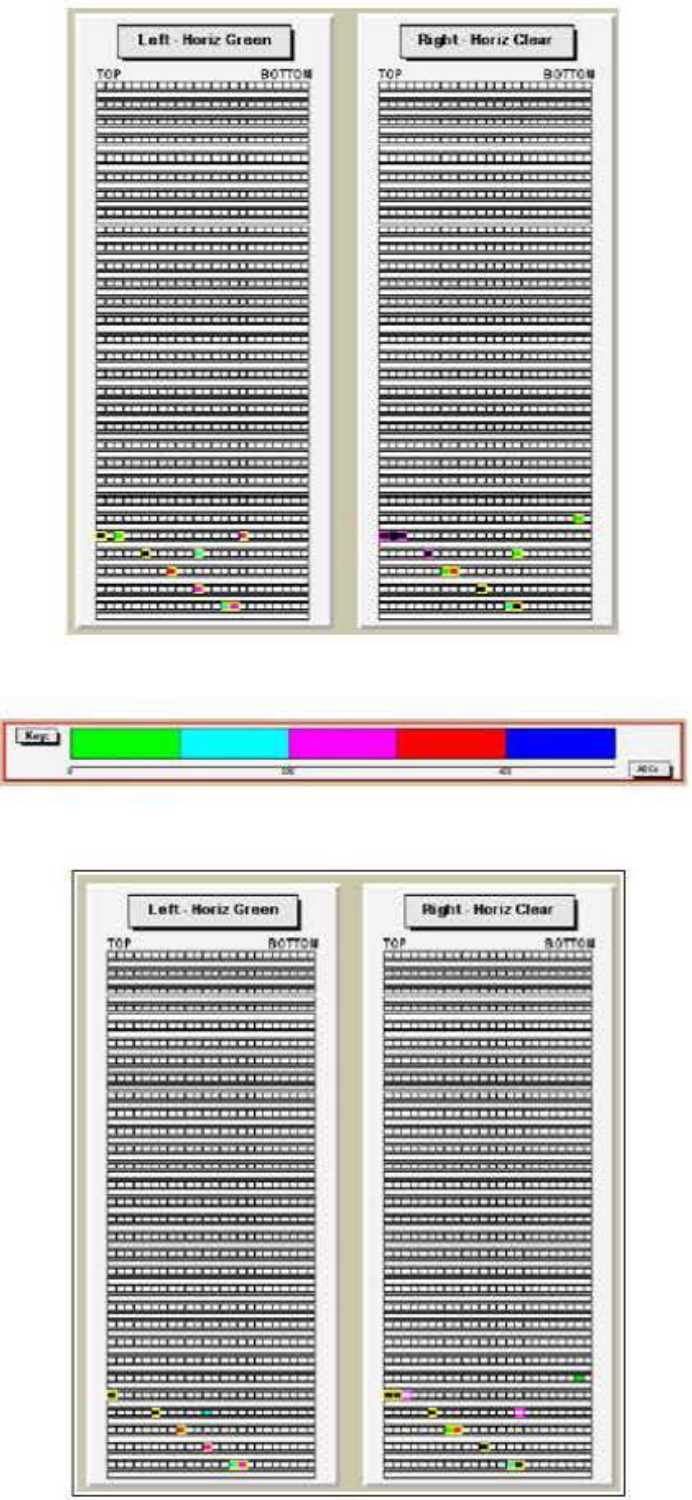

Figure 5.8: Yellow bordered hits are those that have been selected as being part of a track. The top plot is before crosstalk handing. The tracker has become confused by crosstalk hits. Afterwards, the bottom plot shows the track is identified correctly. 


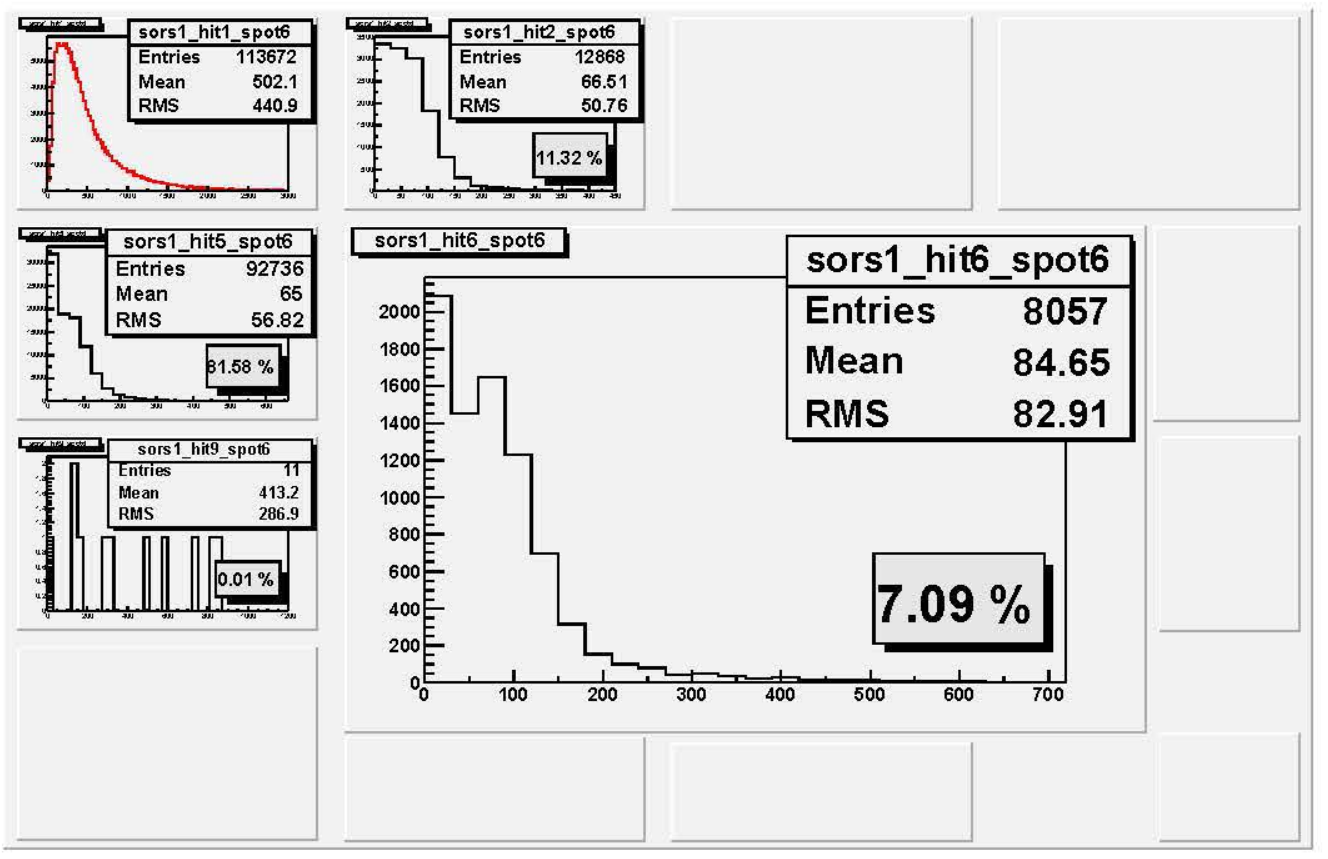

Figure 5.9: Histograms of crosstalk hit size with the ADC value on the $\mathrm{x}$-axis. Crosstalk from pixel 1 (top left, red, source specrum) to other pixels, is shown. The relative percentage of crosstalk hits to each pixel is shown. The plot of crosstalk to pixel 6 has been blown up to make the 1 p.e. peak clearer. No crosstalk is seen in the other 11 pixels of the PMT face.

would be required; for M16s this seems reasonable but may not be adequate for M64 crosstalk removal code [86].

The top plot of Figure 5.10 shows a cosmic ray muon track that a number of crosstalk hits have been identified with. Only crosstalk hits that go from the source pixel to the 8 nearest neighbouring pixels are considered. By eye, it's clear that the vast majority of crosstalk has been succesfully identified. The only apparent case that a potential crosstalk hit has not been found is in plane 25 in the plot labelled 'Right - Horiz Clear'. The mux box readout for the pair of planes associated with this hit is shown in the bottom plot of Figure 5.10 and the grey pixels indicate the middle PMT where inter-plane crosstalk can occur. On the left PMT can be seen a clear case where a large hit (>500 ADCs) has generated a crosstalk hit into the pixel below it ( $\angle 100 \mathrm{ADCs}$ ). On the right, it is far less clear how the hit should be handled. The relative difference in size of the two hits (which is normally a good 

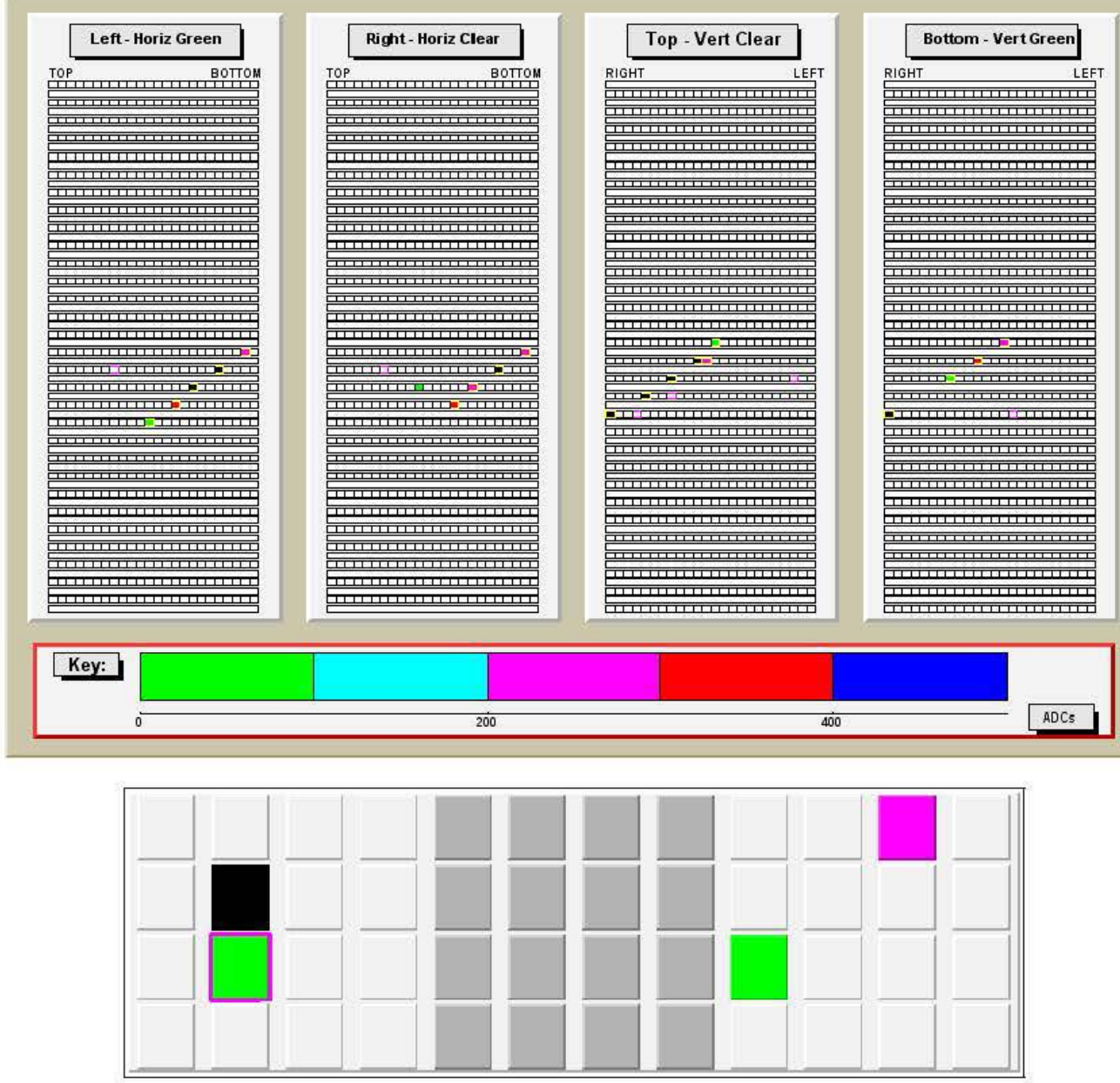

Figure 5.10: This event display shows a typical cosmic mon signature in the detector. Hits identified as being part of a tarack are bordered in yellow and prospective crosstalk hits are bordered purple. The 3 PMTs of the mux box corresponding to Plane 25/27 on one side is shown. The grey pixes denote the middle PMT where inter-plane crosstalk can occur.

indication of crosstalk) is less than for the other case (100-200 ADCs) and they are separated by a large distance. The entromment of CaDDet is quite noisy with contaninants such as neutrons causing spurions hits as well as crosstalk. Since this hit camnot be unambiguously identified as any one of these, it is let untagged. In this cass the hit has not been tracked anyway, so the effect of leaving it is not great.

In Figure 5.11 it can be seen from the number of entries in the warious histograns that the majority of crosstalk goes to the pixels directly above and below and to the 


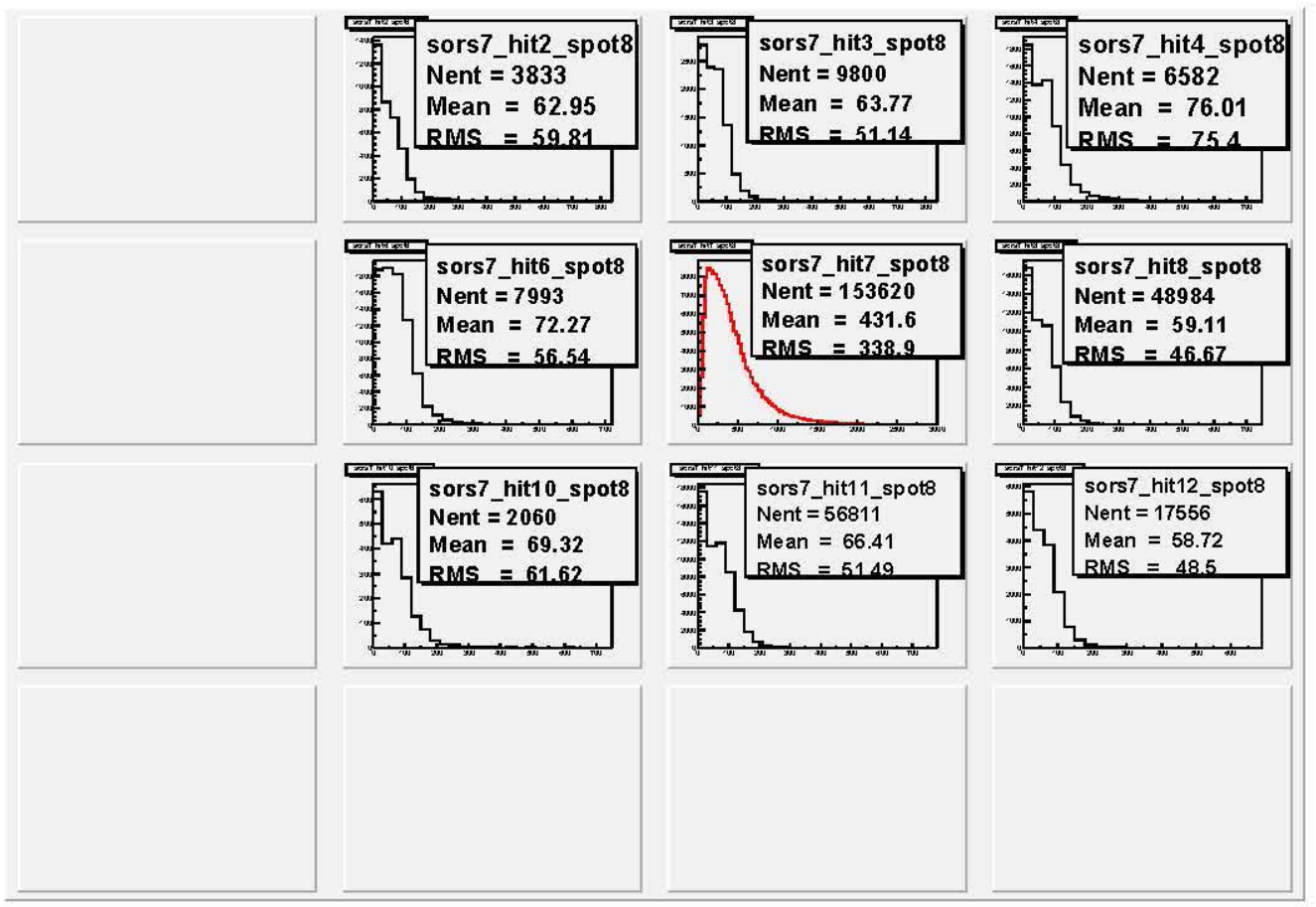

Figure 5.11: The face of a single PMT. The quantity of crosstalk seen on other pixels from pixel position 7 (red, source spectrum), with light injected at spot 8 . The ADC value is plotted on the $\mathrm{x}$-axis in each case.

side of the source hit. Diagonal crosstalk is less frequent. The relative distribution is heavily dependent on the spot position; in the case shown, the pixel is receiving light along the optical fibre from spot 8 which is in the bottom right hand corner.

It is also thought that the distribution of optical crosstalk is modified in a west to east direction by the 'Venetian Blind' structure of the dynode chains [88]. This small effect is difficult to observe in the data, particularly since the fixed spot-to-pixel configuration does not easily lend itself to a pixel cross-comparison.

Figures 5.12 and 5.13 show crosstalk hit ADC against the ADC value of the source hit. Figure 5.12 gives all the crosstalk hits seen from pixel 6 . The thick band of hits along the bottom of each pixel plot appears to suggest that the electrical crosstalk hit ADC is not strongly correlated with the source hit's ADC. The plot in pixel 8 is the $y$-projection of the crosstalk in pixel 7 , again highlighting the 1 p.e. peak. The top plot of Figure 5.13 shows only the crosstalk produced in pixel 3 (spot position 4) that appears in pixel 4. The colour represents the number weighting. 


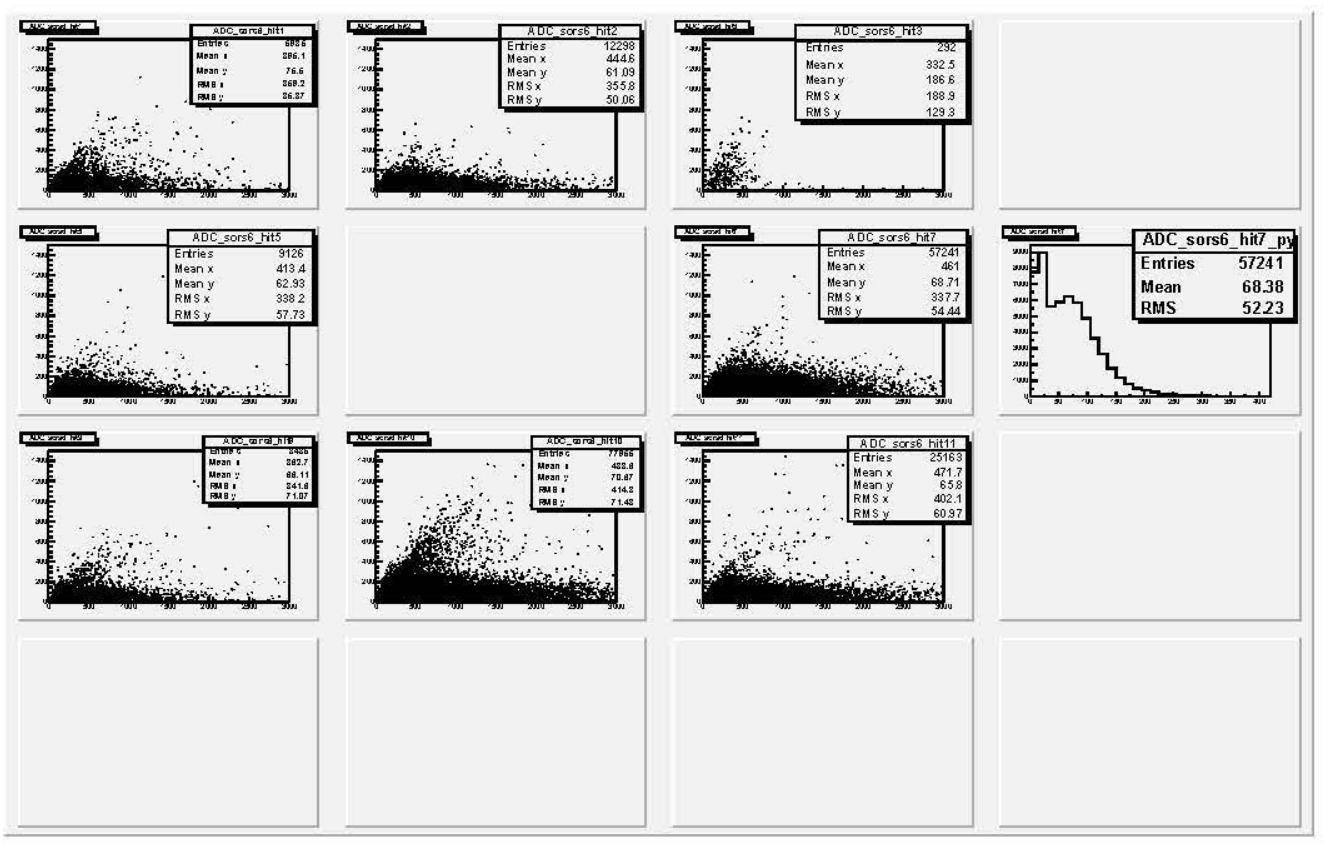

Figure 5.12: Hit ADC vs Crosstalk ADC resulting from hits in pixel 6. The plot in pixel 8 is the $y$-projection of the crosstalk in pixel 7 , highlighting the 1 p.e. peak.

Underneath are the $\mathrm{x}$ and $\mathrm{y}$ projections which represent the input(source) and output(crosstalk) spectra respectively. There only appears to be a small indication of correlation between the size of a hit and its resulting optical crosstalk hit. This may be at least partly due to the fact that the input spectrum is highly peaked at the low ADC range where the majority of muon energy deposits lie. ${ }^{2}$

Some indication of the quantity of crosstalk seen is shown in Figure 5.14. The plot shows the number of crosstalk hits divided by the total number of hits on an event by event basis in each pixel of a PMT. It represents the average number of crosstalk hits per real hit. The discrete spikes at $0.5,0.333,0.25$ indicate a very high crosstalk to hit ratio; these are indicative of large, low multiplicity hits at the front of the detector caused by splattering particles. A good estimate of the amount of crosstalk seen can be found by fitting to the peak around 0.14 in which case around 1 hit in 7 is crosstalk. Looking across the wider spectrum of events as in Figure 8, this increases to 1 in 6 .

In Figure 5.15, crosstalk ADC divided by source ADC is shown. The distri-

\footnotetext{
${ }^{2}$ The crosstalk algorithm was primarily designed to assist with muon event reconstruction
} 

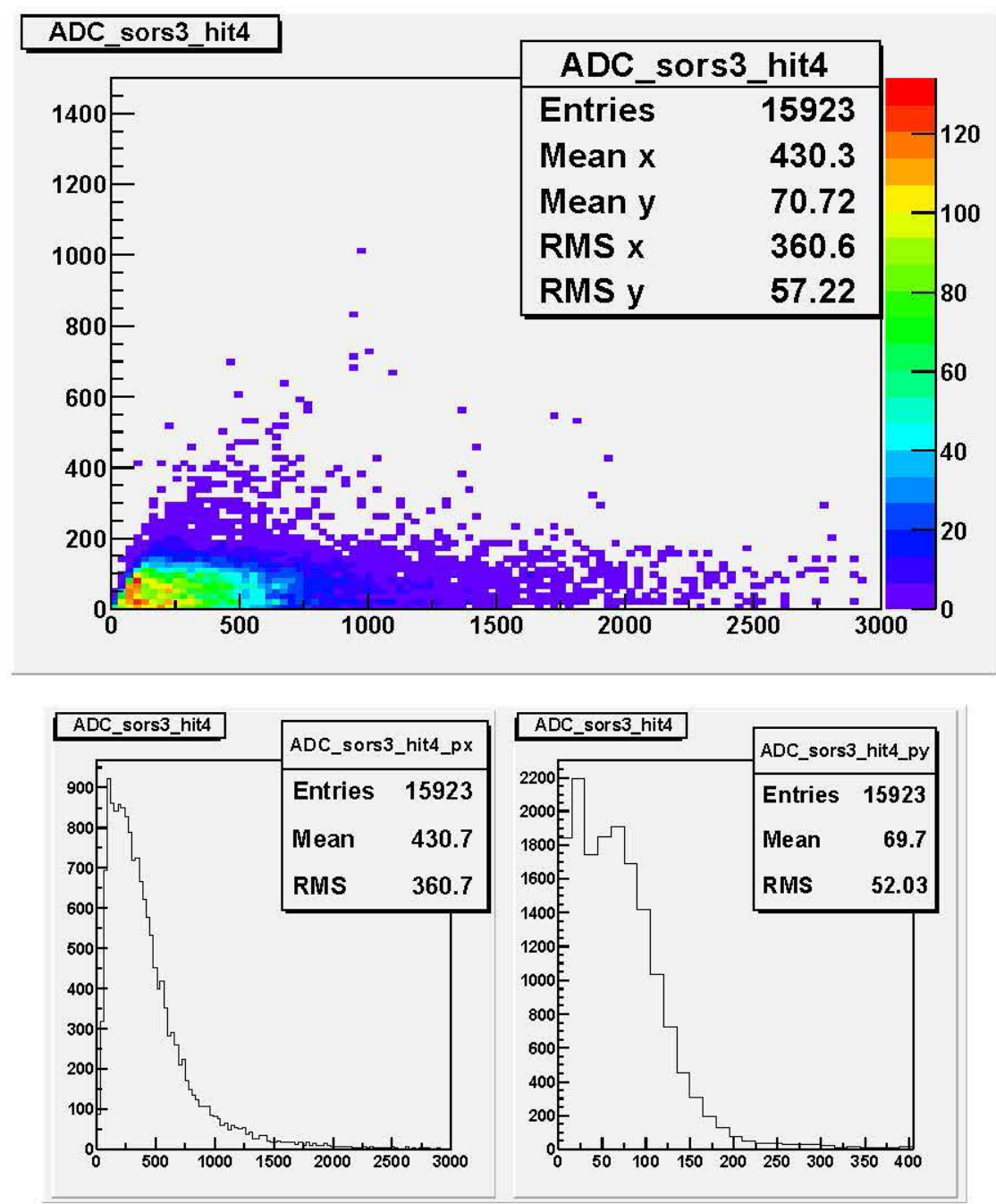

Figure 5.13: Top: Number weighted crosstalk ADC (y-axis) vs source hit ADC (xaxis). Bottom left: Input source spectrum (x-projection of above plot). Bottom right: Output crosstalk spectrum (y-projection of above plot, zoomed in)

butions fall off exponentially as is probably to be expected indicating that most crosstalk is at a very low level. What is maybe somewhat more surprising is that $\sim 2 \%$ of events shown here are larger than 1 indicating that the crosstalk hit is larger than the original source hit. This seemingly unlikely occurence is possible at low light levels due to the fact that the electron multiplication at each dynode has a gaussian form. If a similar number of photoelectrons end up in the crosstalk dynode chain as in the correct dynode chain, they can be multiplied at differing rates producing optical crosstalk signals comparable with the original hit. 


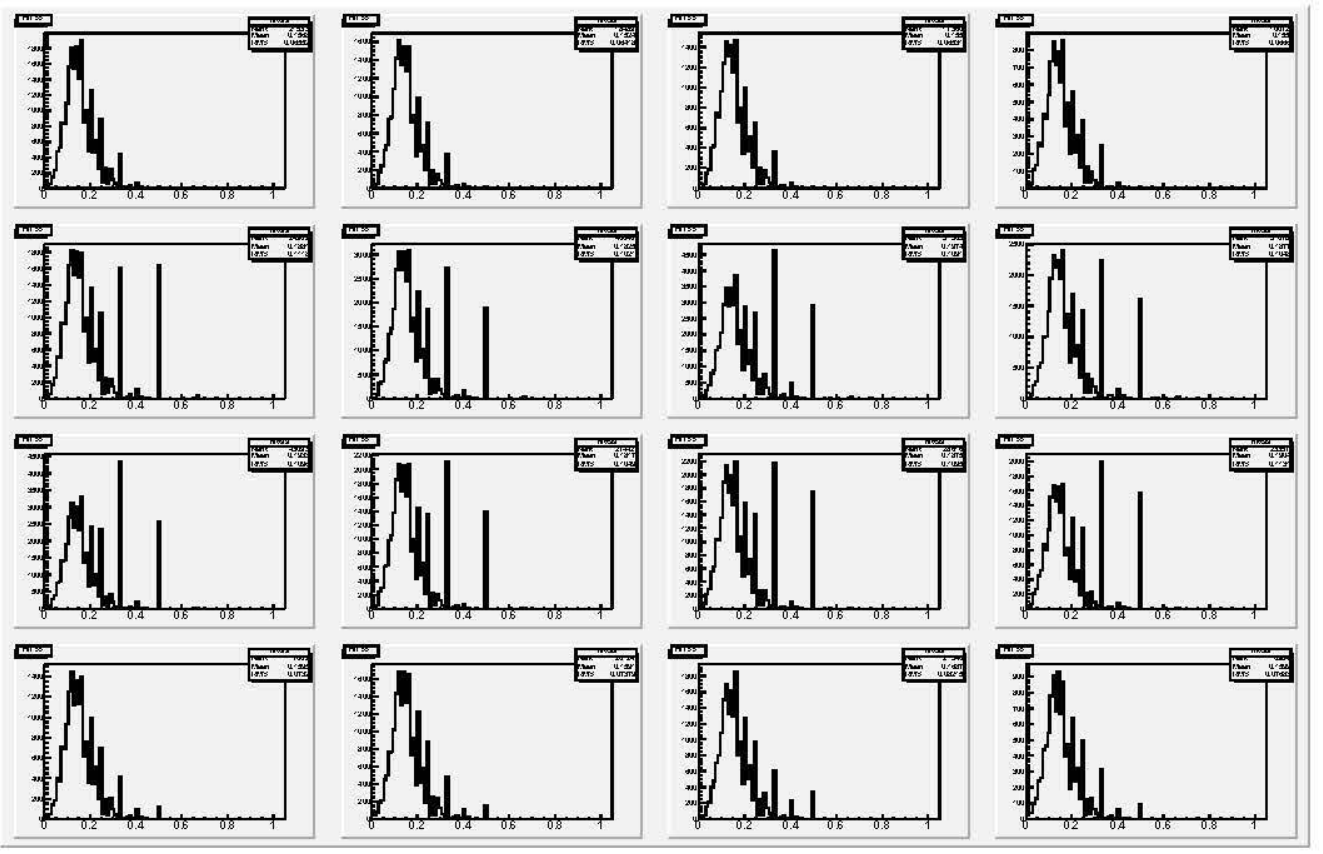

Figure 5.14: Number of crosstalk hits divided by the total number of hits per event.

The pixel spot illuminated in this case is 8 ; it can clearly be seen that the number of crosstalk hits in the pixels closest to the source is larger than the number far from it, as expected.

\subsubsection{Crosstalk Removal Algorithm}

The crosstalk removal algorithm (Crosstalker) runs as a module before the CalDet muon tracking code [85]. It is designed to 'clean up' the crosstalk hits before they are passed to the main body of the tracking code. The crosstalker performs the following steps when executed:

- Moves through all the hits in a (mux box connected) plane-pair in turn

- Looks at the 8 pixel window around each 'test hit' for other hits

- Finds the largest hit in the window and also sums the 8 pixel charge

- The test hit is flagged as crosstalk if its charge, Qtest $\leq \alpha \times \Sigma Q_{\text {window }}$ where $\alpha$ is a user definable variable which can be adjusted according to the particle type under investigation; it should be set around 1 for low energy deposition 

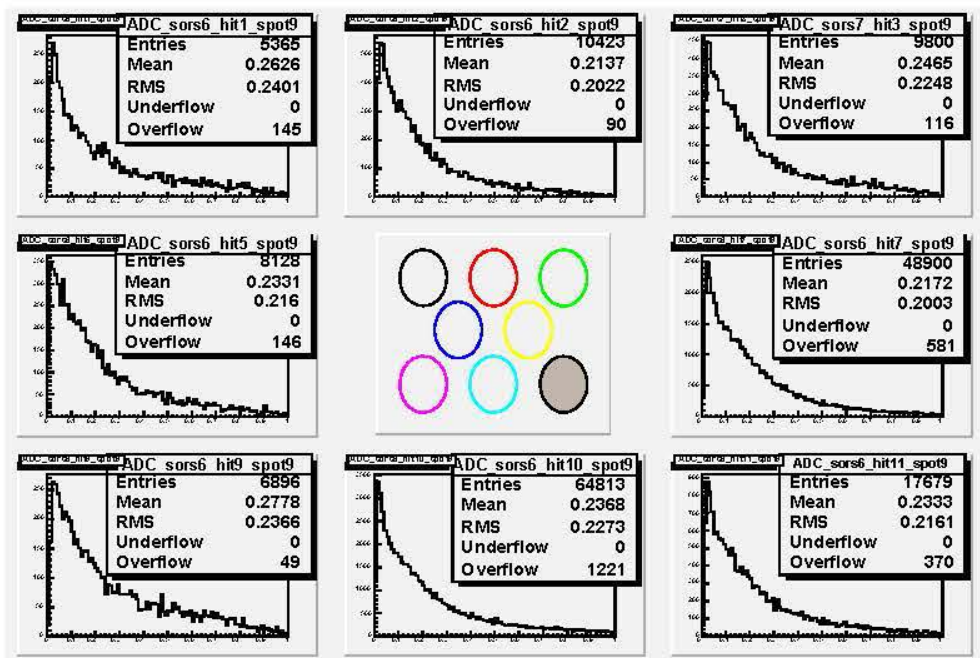

Figure 5.15: Crosstalk ADC/Source ADC from pixel 6 with light coming in at spot 8.

and low density of hits on the PMT face (i.e. muons) and much lower for high hit density and high energy deposition (i.e. electrons)

- A test hit is then unflagged if there is a hit in the same strip of the next or previous plane in the case of beam particle tracking, or in any of the 3 strips ahead of or behind in planes in the case of cosmic muon tracking. The hit is also disregarded if it is found in a strip directly adjacent to an existing hit. ${ }^{3}$ These 'veto window' cut conditions are a little severe, but remove the undesired possibility of a genuine hit being assigned as crosstalk and hence not being passed to the tracking code.

- The algorithm then moves to the next plane-pair and repeats until all planes in the detector have been analysed.

\footnotetext{
'This is to prevent 'corner-clipping' hits from being mistakenly identified as crosstalk, See [84] for more on comer-clippers.
} 


\subsubsection{Algorithm Performance}

In order to assess the effectiveness of the code, it was tested on Monte Carlo with crosstalk fully simulated. Figure 5.16 shows the results. The top left plot shows the distribution of crosstalk in the detector according to the Monte Carlo truth. The top right plot shows the hits identified by the Crosstalker which were subsequently sent to the Tracker for processing and output. For the most part there is good agreement, with $82 \%$ of the crosstalk hits being successfully marked as being consistent with crosstalk. The crosstalker always errs on the side of caution and leaves hits that cannot be identified within reason, so the majority of the remaining $18 \%$ were complicated situations (such as multiply scattered muons that produced crosstalk in adjacent pixels to the track or other occasions where genuine crosstalk fell into the 'veto window' around a hit).

Figure 5.17 demonstrates the importance of crosstalk removal. The plots show the reconstructed ranges (stopping distance) of muons at CalDet. The beam energy was set to $2.0 \mathrm{GeV}$ for the runs analysed and muon selection cuts were applied. The top plot shows the ranges without crosstalk handling and the middle plot shows the ranges for the same data but with crosstalk removed. The total number of entries in these plots show that an additional $4.6 \%$ of muons are successfully reconstructed. More importantly, the muon peak is shifted backwards by around 0.5 of a plane. Muons travelling though CalDet are described by the Bethe-Bloch formula, and exhibit increased energy deposition as they are about to stop. These larger hits are more likely to produce crosstalk than smaller hits earlier in the track and so more crosstalk is expected towards the end of the track than the beginning. Muon range is a crucial aspect of the MINOS calibration, so it is essential to remove the crosstalk which is artifically extending the tracks via the inter-plane crosstalk effect described earlier. Crosstalk removal is applied to all subsequent analyses in this work. Muon $\mathrm{dE} / \mathrm{dX}$ in CalDet is fully discussed in Chapter 5.3. 

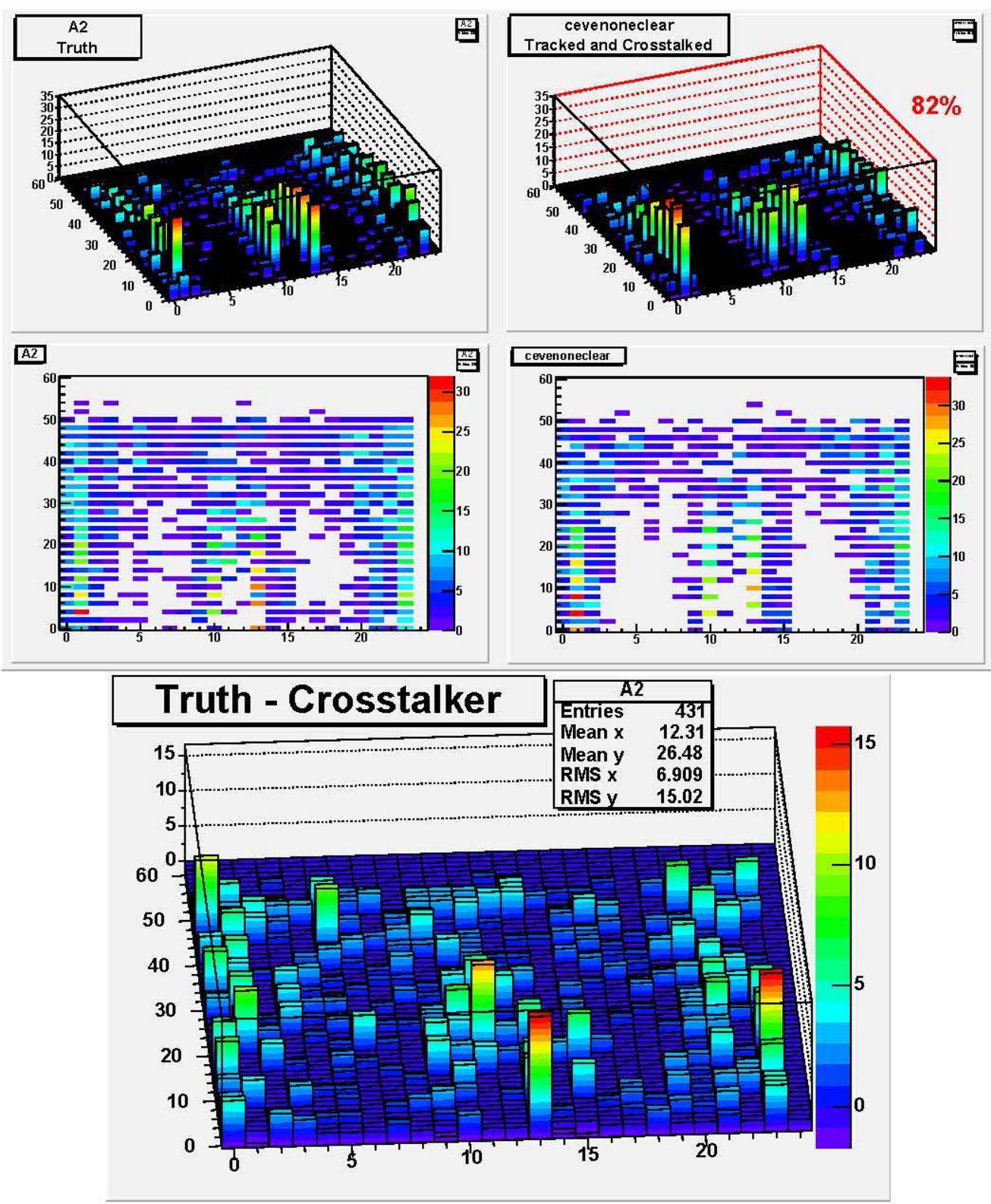

Figure 5.16: The distribution of crosstalk hits seen throughout the detector are plotted. The left plots are the truth and after crosstalking and tracking is shown on the right. The middle two plots represent the $\mathrm{x}$ and $\mathrm{y}$ projection of the top plots. The bottom plot shows the subtraction of the right plots from the left ones. 

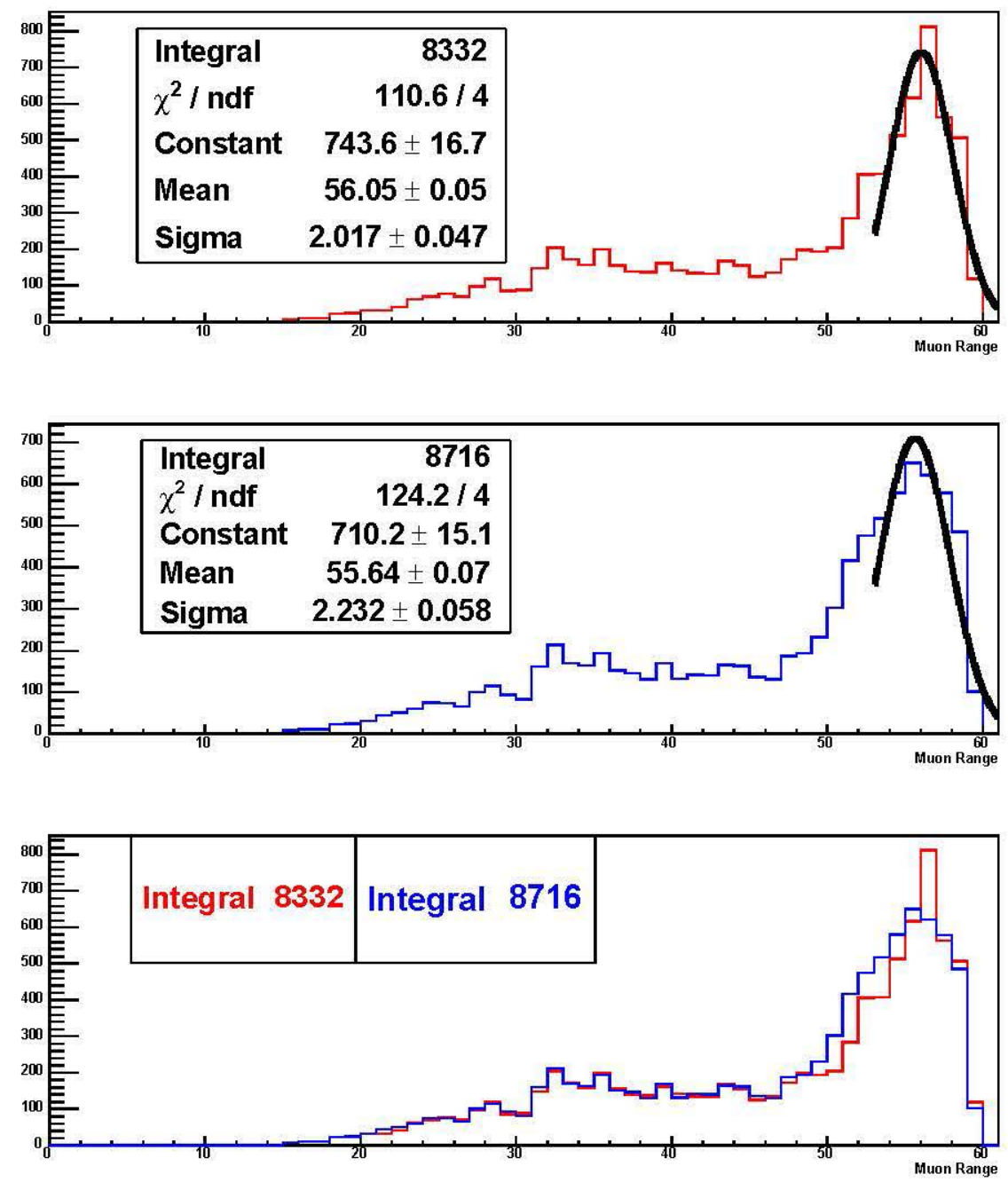

Figure 5.17: Top: The muon range for raw data, with muon selection cuts applied. Middle: Muon ranges for the same dataset but with crosstalk removal. Bottom: The top and middle plots overlaid. The crosstalk removal algorithm improves tracking efficiency slightly and identifies forward going inter-plane crosstalk hits that frequently appear at the end of muon tracks. Crosstalk hits are more common at the ends of muon tracks because energy deposition is typically higher there. The muon sample was produced with a beam energy setting of $2.0 \mathrm{GeV}$. 


\subsection{Summary}

Calibration of the MINOS detectors is a complex but essential procedure. The electronics are calibrated using known quantities of charge that are injected and read out. The response of each pixel on every PMT is homogenised using the light injection system than supplies a known quantity of light to each element. Production and quality differences in the MINOS scintillator strips are accounted for using cosmic ray muons that deposit a constant amount of energy in each strip they pass through. Events in the different MINOS detectors can be compared like to like by calibrating with stopping cosmic muons (or beam muons in the case of CalDet) which travel the same distance through all the detectors, for a given muon energy. Finally, stopping muon ranges can be translated to absolute energy as a result of the studies of beam muons at CalDet. The energies of beam muons are known a priori, this is not the case for muons seen in the Near and Far detectors.

PMT crosstalk is another important calibration issue for the MINOS experiment. Crosstalk can cause errors in the particle tracking codes and artificially increase the ranges of these particles. The characteristics of crosstalk in the data have been studied. The relative importance of electrical and optical crosstalk has been considered, and it has been determined that the spot and pixel positions have an effect on the amount of crosstalk observed in any given pixel. It has been found that around 1 hit in every 7 is crosstalk. A crosstalk removal algorithm has been developed which is successful at removing $82 \%$ of crosstalk hits. This results in an $4.6 \%$ improvement in tracking efficiency and pulls the muon stopping range back by 0.5 a plane at CalDet. 
chapterMuon $\mathrm{dE} / \mathrm{dX}$ in the Calibration Detector

This chapter is focussed on the identification of muons, and the measurement of muon $\mathrm{dE} / \mathrm{dX}$ in the Calibration Detector. The main characteristic of $\nu_{\mu}$ events in the MINOS detectors, and the main tool for measuring the oscillation parameters, is the identification and measurement of the muon which is produced in a charged current interaction of a $\nu_{\mu}$. The energy of the NuMI beam is rather low (about 2 $\mathrm{GeV}$ ) and the main background to $\nu_{p}$ events is from neutral current events where a pion is produced which simulates a muon by travelling a significant distance before interacting. Understanding how these events differ from real muons in the detector is a very important issue and another is the estimation of the irreducible background from them. Probably the main tool used to measure muon momentum in the MINOS detectors will be range (although there is a magnetic field), and a thorough understanding, and measurement of the muon $\mathrm{dE} / \mathrm{dX}$ will be paramount in view of the fact that the whole MINOS calibration hinges on comparing muons of similar energy in all three detectors. At this energy, the muon $\mathrm{dE} / \mathrm{dX}$ is not very well known, and large differences between data and Monte Carlo have been identified.

A clean sample of muons at CalDet is first identified using shape and energy cuts or cerenkov detectors. The detectors have an implicit efficiency, which is dependent on the muon energy and needs to be calculated, in order that it can be corrected for. Other detector effects also have to be taken into account: differences in pressure between the cerenkovs and pions decaying to muons between them both skew the observed efficiency. Once the true efficiency is found, the observed muon spectrum is corrected to obtain the true muon energy spectrum of the test-beam.

\subsection{Pion/Muon Separation}

High energy charged current $\nu_{p}$ events in the Near Detector and Far Detector can easily be detected by their characteristic 'long-event' nature; a muon created at the interaction point which produces a long track of hits that traverse some or all of the distance to a detector edge.

Neutral current events can produce pions. These tend to interact hadronically, producing short, shower like events similar to those produced by electrons. Occa- 
sionally however the pion will not interact hadronically but instead travel through a medium, slowly depositing energy through ionisation as it goes. In these cases, pions can look very similar to muons.

Two methods are used to separate muons and pions. The first involves cerenkov detectors that are available in the test-beam environment of CalDet. The second method uses shape and energy cuts that are tuned by Monte Carlo simulation.

\subsection{Muon selection using the Čerenkov Detectors}

At CalDet, unlike the other detectors, there is a method by which pions and muons can be distinguished with a high degree of certainty. Just upstream of CalDet were located between one and three Cerenkov detectors (depending on the beam line and the year). Put simply, a cerenkov detector is a sealed aluminium tube filled with a variable quantity of gas. Energetic particles travelling through them can possess a velocity greater than the local speed of light and produce photons of cerenkov radiation which are detected by a photomultiplier tube at the end of the counter. By pumping the cerenkov detectors to certain pressures using $\mathrm{CO}_{2}$, it was possible to set them such that a pion travelling through them would not produce enough cerenkov light to trigger them, whilst a muon that travels slightly faster for a given momentum, would. Figure 5.18 shows the pressure-momentum thresholds for pions, muons and kaons. Electrons always fire the detectors unless the momentum and pressure are very low.

In the $T 7$ beam line in 2003 , a series of runs were taken in which there were two operational cerenkov detectors. The more upstream counter was $4.4 \mathrm{~m}$ long and is referred to as the 'upstream cerenkov' or 'Cerenkov 3'. The counter closer to CalDet was $3.5 \mathrm{~m}$ long and is referred to as the 'downstream cerenkov' or 'Cerenkov 1'. A second detector originally lay between them but was removed because it was not functioning properly. Both cerenkovs were pumped up to 4.4 atmospheres which, at $2 \mathrm{GeV}$, ought to distinguish pions from muons. This was the maximum pressure that could be applied to the detectors and so $1.8 \mathrm{GeV}$ muons and pions also had to be studied at this pressure. Whilst this was closer to the muon pressure threshold, $1.8 \mathrm{GeV}$ muons are also easier to distinguish from through-going PS muons than 


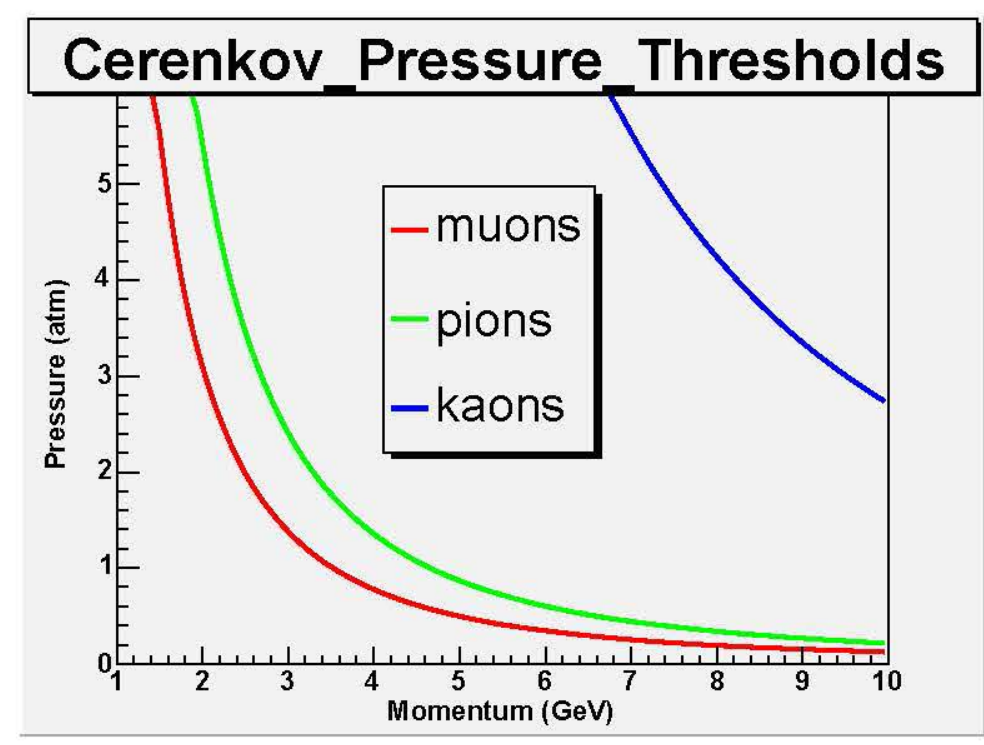

Figure 5.18: Diagram showing the Cerenkov pressure thresholds for particles of various momenta using $\mathrm{CO}_{2}$. The areas above each line are the regions of space where the particle is above the triggering threshold of the cerenkov detectors. When the beam is set to select particles of say $3 \mathrm{GeV}$, it can be seen that at 2 atm, muons will fire the detector and pions will not.

those at $2 \mathrm{GeV}$. Figure 5.19 shows this. The data has been broken down into four categories: events that fired both cerenkovs, events that fired neither and events that fired only one or the other. It is expected that events that fired both cerenkovs must be muons or electrons and those that fired neither be pions. For each of these categories, the maximum depth the particle travels into the detector is shown. This quantity, defined as the range of the muon is measured in units of planes, but an event with a range of 50 has not necessarily reached the 50 th plane of the detector because it may have undergone multiple scattering. The top left plot (both cerenkovs fired) shows peaks at ranges of around 51 and 60 , corresponding to $1.8 \mathrm{GeV}$ beam muons and PS muons respectively. Electrons cause short, shower like events in the detector that do not penetrate very far; the width of these events would be expect to be much larger than that of muons, but that quantity is not shown here. The $1.8 \mathrm{GeV}$ beam muons lose energy as they travel through the detector and finally stop after about 51 planes. There is some natural spread in the momentum of these muons and they sometimes multiply-scatter as they traverse the detector, resulting 

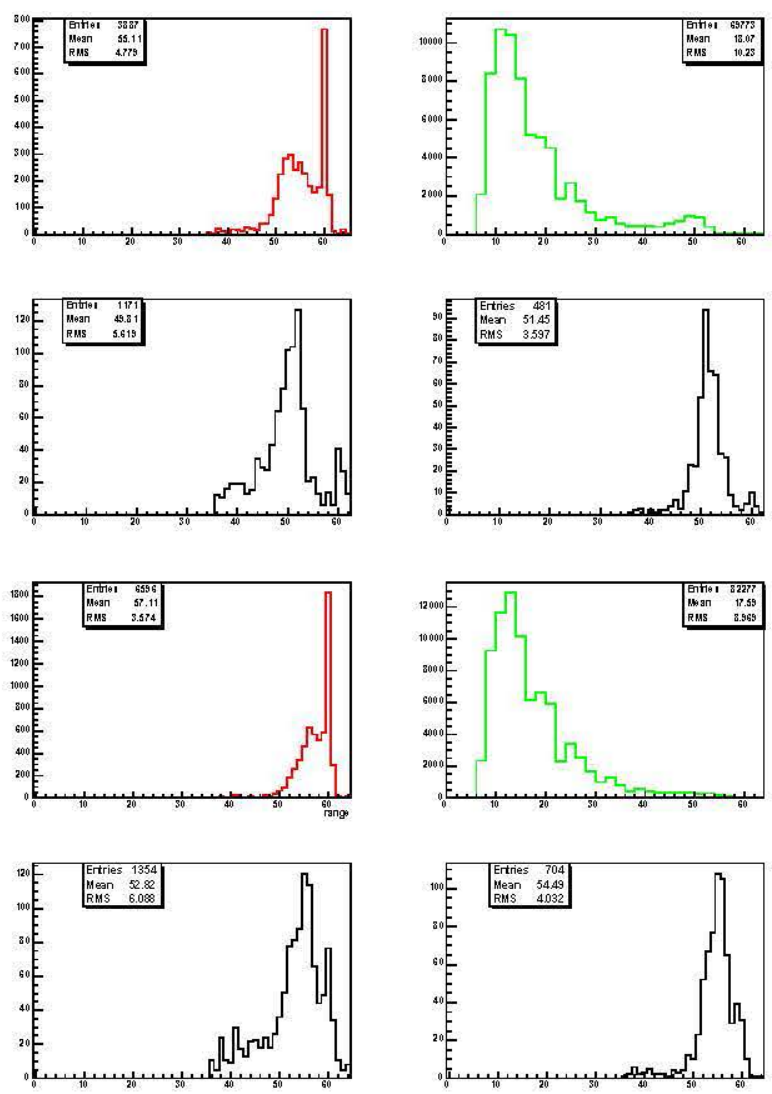

Figure 5.19: Plots of particle range for events selected by the cerenkov counters. The top four plots are for $1.8 \mathrm{GeV}$ and the bottom four for $2.0 \mathrm{GeV}$. In each of these groups the top left plot shows events that fired both cerenkovs; these are muons, but a significant amount of contamination from PS muons is seen, especially at 2.0 GeV. This is observed as the peak at range 60 , indicating particles that have travelled straight through the detector. The top right shows events that fired neither cerenkov counter. In the bottom left are events that fired the upstream counter but not the downstream one and the bottom right depicts events that fired the downstream counter but not the upstream.

in the relatively large spread of final ranges. PS muons tend to be highly energetic and travel right the way through the detector in a relatively straight line. The most important feature of Figure 5.19 is that the peak range of $2.0 \mathrm{GeV}$ muons is much closer to the PS muon peak than for $1.8 \mathrm{GeV}$ muons, and since the PS muons are effectively a background to a pure on-momentum muon sample, the $1.8 \mathrm{GeV}$ events 
are 'cleaner' to begin with.

The top right plots show the ranges of the particles that fired neither cerenkov and so are expected to be pions. There is a significant peak around 15 planes where the pions are interacting quickly and hadronically, but some fraction of them behave like minimum ionising particles and continue much further on, in the same way as the beam muons.

Another way to look at the muon and electron sample is a plot of range verses the cerenkov signal, see Figure 5.20. If can be seen that low ranged, high velocity electrons produce a larger signal in the detectors than the slower beam muons. A high density of events can be seen at a range of 60 and are mostly PS muons. Being of higher energy, they do generate a signal in the cerenkovs that is on average larger than normal beam muons, but the samples are not sufficiently separated for this to provide a reliable cut parameter. At this energy, electrons never travel more than 30 planes, which provides a convenient way of removing them. Along the bottom of each graph can be seen a high density band of events that registered zero ADC counts in the cerenkov detectors that did trigger the TOF and hence the read-out. These are the pions.

In order to remove PS muons from the beam muon sample, fiducial cuts are imposed on the data. Any muon that multiply scatters and leaves the detector prematurely (i.e. out of the side) is excluded as its momentum cannot be determined from its range. Beam muons however cannot travel further than their momentum allows, hence a $2.0 \mathrm{GeV}$ beam muon cannot leave the side of the detector any later than plane 55. Thus any muons that leave the edge of the detector past plane 55 or

leave via the last plane are deemed to be PS muons. Figure 5.21 shows the effect of the fiducial cut.

\subsubsection{C̈erenkov efficiency}

The fact that some particles fire one cerenkov and not the other shows that the detectors are not $100 \%$ efficient. However, the presence of two cerenkov counters allows the efficiency of the first detector to be calculated as follows: 

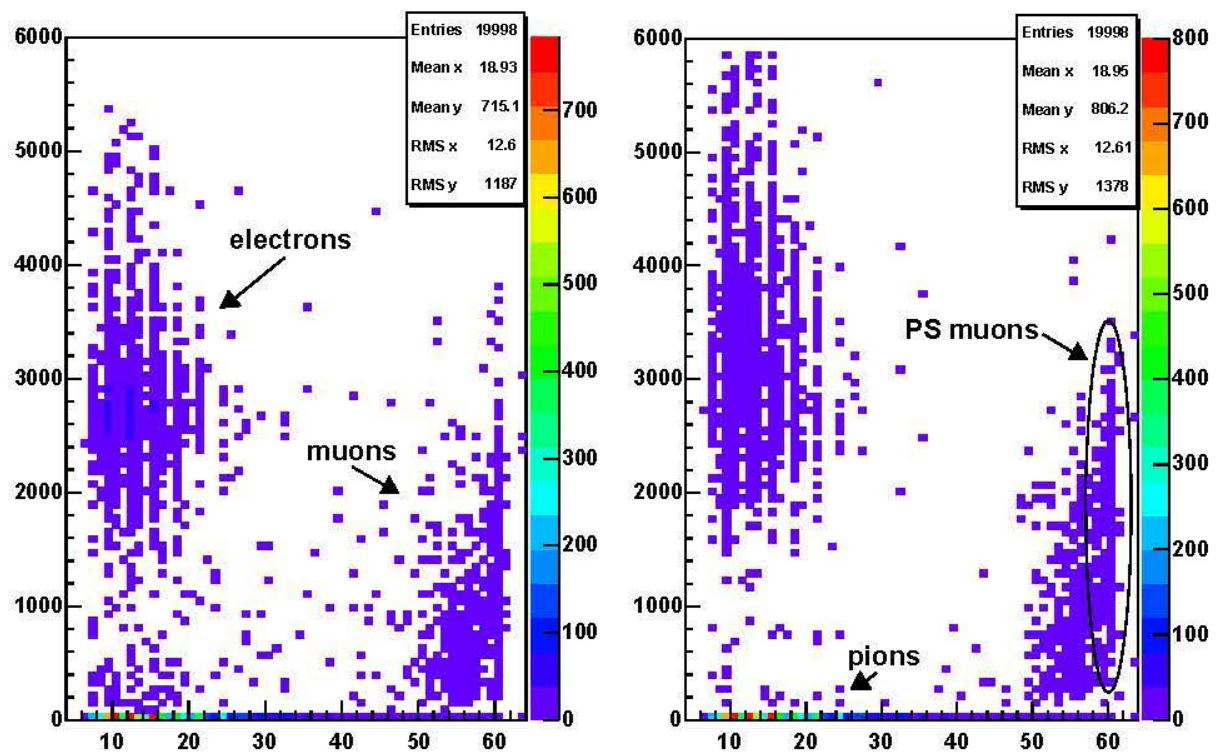

Figure 5.20: Range verses cerenkov adc for events that fired the first counter (left) and the third counter (right). Low range, high deposition events are electrons, long ranged events are muons. The high concentration of events with a range of 60 are caused by PS muons. Note the significant number of events where the adc is zero, these are pions, and muons lost to cerenkov inefficiency. The sum of the x-projections of these plots is equivalent to the top right plot of Figure 5.19.

$$
\frac{3 \overline{1}+\overline{13}}{1 \overline{3}+3 \overline{1}+13+\overline{13}}
$$

And the efficiency of the third detector can be found with the equation:

$$
\frac{1 \overline{3}+\overline{13}}{1 \overline{3}+3 \overline{1}+13+\overline{13}}
$$

where the equation elements refer to the number of events seen, the 1 or 3 refers to counters 1 or 3 and an overbar indicates a 'not'. Therefore ' $3 \overline{1}$ ' would mean 'the number of events seen in counter 3 that were not seen in counter 1'. The quantity $\overline{1} \overline{3}$ is unknown but can be calculated iteratively. The results are shown in Table 5.2. As might be expected, the shorter of the two detectors (the downstream one) is less efficient since particles have less gas to go through and therefore produce fewer photons. 


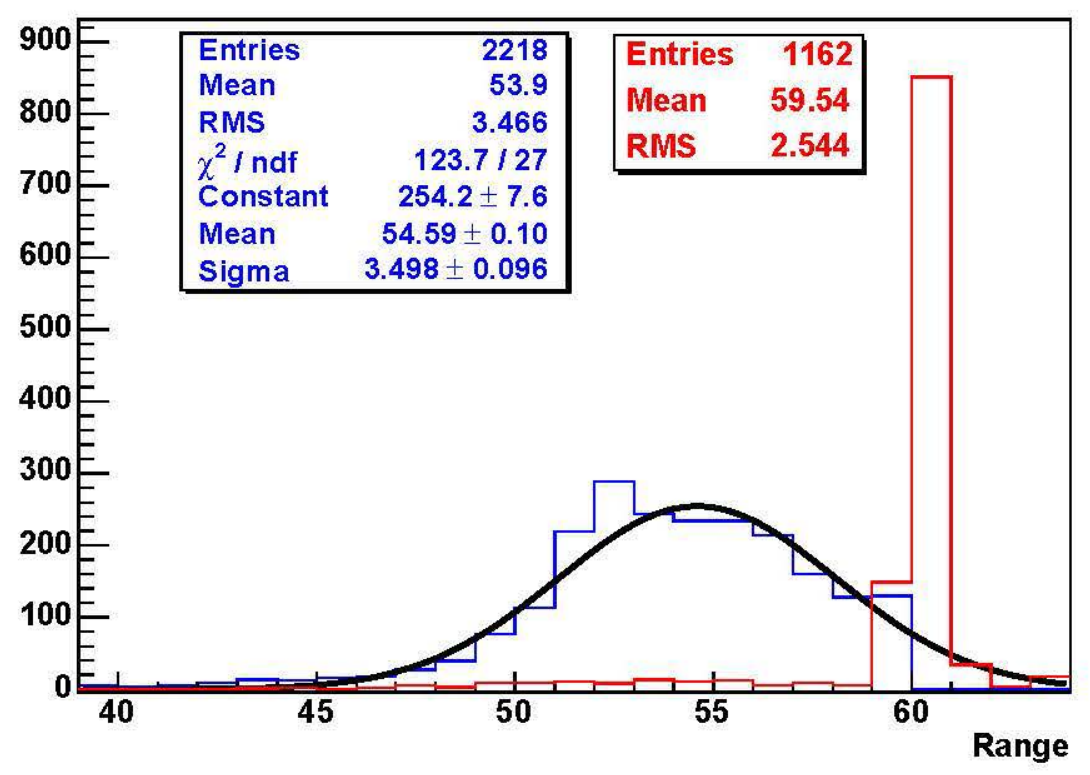

Figure 5.21: The effect of the fiducial cut is to produce a cleaner beam muon selection. PS muons are shown in red and beam muons in blue. A fit is made to the cleaned $2.0 \mathrm{GeV}$ sample.

\begin{tabular}{|c|c||c|}
\hline Muon Energy & Cerenkov 1 Efficiency & Cerenkov 3 Efficiency \\
\hline $1.8 \mathrm{GeV}$ & $74 \%$ & $89 \%$ \\
$2.0 \mathrm{GeV}$ & $88 \%$ & $90 \%$ \\
\hline
\end{tabular}

Table 5.2: The calculated efficiencies of the Cerenkov detectors used in the T7 beam line at CERN. Cerenkov 1 is the shorter, more downstream counter and Cerenkov 3 is the longer, more upstream counter.

The stronger signals in the cerenkov counters from PS muons compared to beam muons shows that the higher the momentum of the particle, and hence the faster it is travelling, the more likely a particle is to set off the cerenkov detectors. Across the intrinsic momentum spread of the particles in the beam, the lower energy muons are therefore less likely to fire the cerenkovs. Since muons are minimum ionising particles and deposit energy at roughly a constant rate, the more energetic, faster muons will penetrate deeper into the detector than the slower ones. Thus the range of the muon can be used as a measure of its momentum, and it follows that the 
efficiency of the cerenkov counters is not constant but depends on the velocity and hence range of the muons. $\beta$ proves to be a useful quantity to plot, being related to a particle's velocity by the equation:

$$
\beta=\frac{v}{c}
$$

and can be seen plotted against the particle's range in Figure 5.22. At 1.8 GeV, the nominal $\beta$ of pions is 0.9971 whilst for muons it is 0.9983 . A pion's expected range is independent of its momentum since it can interact hadronically at any time, but a muon, being a minimum ionising particle is expected to travel further if it has more energy and hence a higher momentum and velocity. The pions are shown here with a $3 \%$ energy spread to reflect the acceptance of the momentum selecting magnets upstream. The plot shows that a muon with only enough energy to take it to plane 39 or less would be indistinguishable from a pion. At 4.4 atm for example, these muons would be highly unlikely to fire the cerenkov detectors at all, or in other words the counters are very inefficient at detecting muons at this energy. It is therefore possible to construct a plot of muon detection efficiency verses range which is shown, for $1.8 \mathrm{GeV}$ muons, in Figure 5.23.

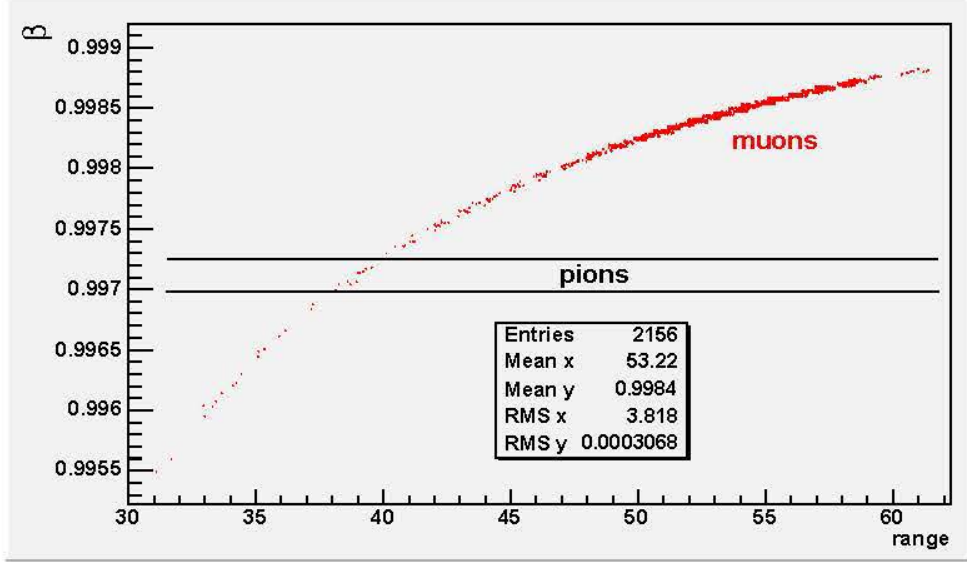

Figure 5.22: Range verses $\beta$ for muons and pions. The distance that a muon travels through the detector is proportional to its energy. This is not the case for pions which can interact hadronically. The pions are shown with a $3 \%$ spread around their nominal energy. Around plane 40, muons and pions have the same beta and so below this they cannot be distinguished by the cerenkovs. 

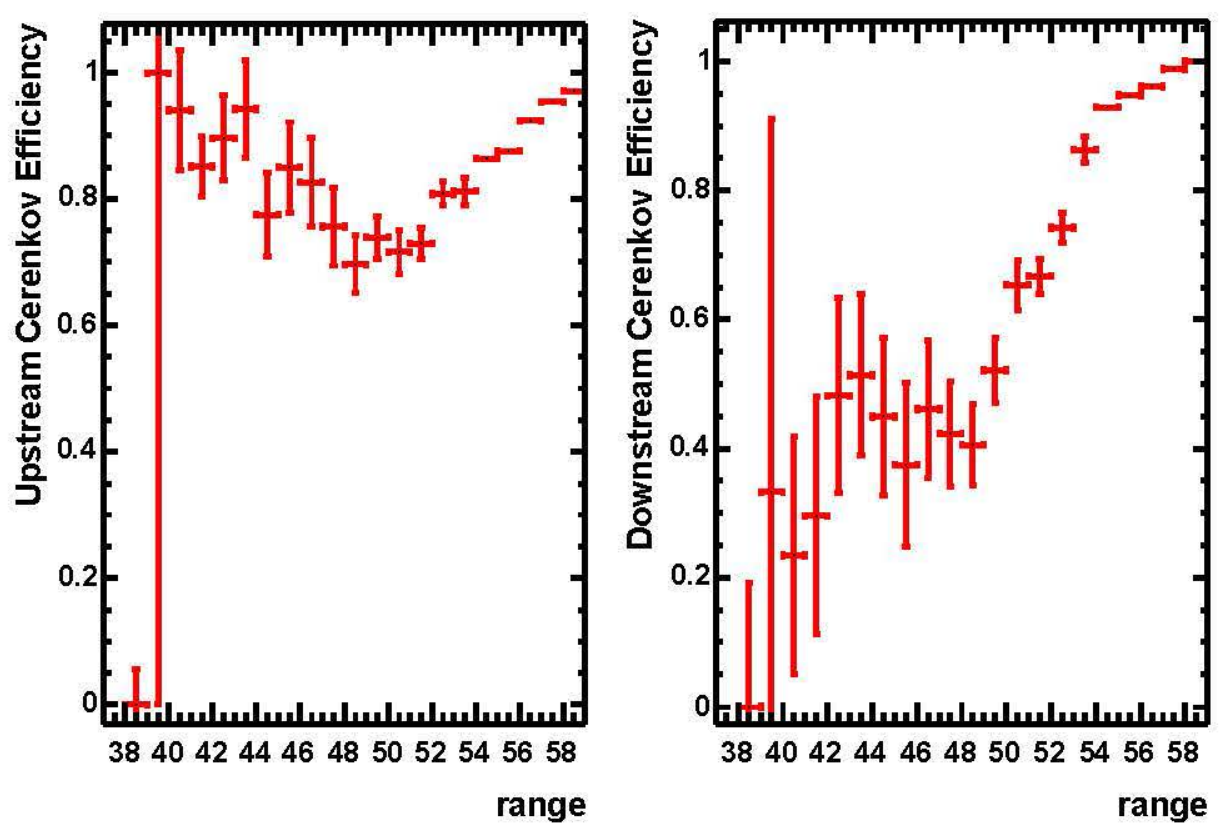

Figure 5.23: The efficiency of the cerenkov detectors on a plane-by-plane basis. Below plane 50 the efficiency is poor.

Cerenkov 1 appears to exhibit some odd behaviour. As the range decreases, the efficiency begins to rise again after the expected dip. To understand this, it is important to note that energy deposition is given by the formula:

$$
-\frac{d E}{d X}=K z^{2} \frac{Z}{A} \frac{1}{\beta^{2}}\left[\frac{1}{2} \ln \frac{2 m_{e} c^{2} \beta^{2} \gamma^{2} T_{\max }}{I^{2}}-\beta^{2}-\frac{\delta}{2}\right]
$$

where $z$ is the charge of the incident particle (in units of e), $\beta c$ is its velocity, $\gamma$ its the relativistic gamma factor, $\mathrm{T}_{\max }$ is the maximum kinetic energy transferable to a free electron in a single collision, $\mathrm{Z} / \mathrm{A}$ is the ratio of the charge number to the mass number of the medium involved, I is its mean excitation energy, $\delta$ is a density effect correction. $\mathrm{K}$ is a constant given by $4 \pi \mathrm{N}_{A} \mathrm{r}_{e}^{2} \mathrm{~m}_{e} \mathrm{c}^{2}$ where $\mathrm{N}_{A}$ is Avogadro's number, $r_{e}$ is the classical radius of an electron and $m_{e} c^{2}$ is the rest energy of an electron.

Taking the logarithm of (1-efficiency) gives the number of photoelectrons (n.p.e.) produced as the particle travels through the counter.

Since $\mathrm{dE} / \mathrm{dX}$ is proportional to $1 / \beta^{2}$, by plotting these quantities against each, 

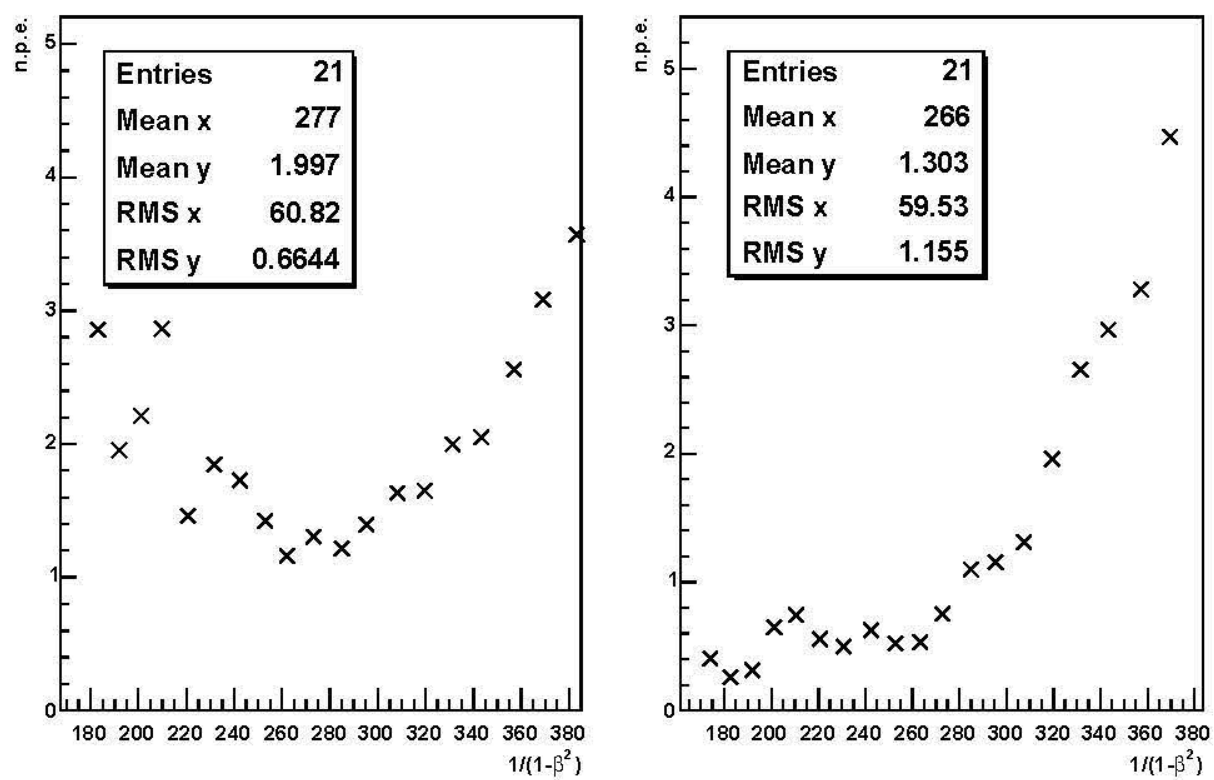

Figure 5.24: $1 / 1-\beta^{2}$ verses the number of photoelectrons. The $\mathrm{x}$-intercept of a straight line fit should give a minimum below which muons are not travelling fast enough to trigger the cerenkov counters reliably. The left plot is crerenkov 1 (downstream) and the right plot is cerenkov 3 (upstream).

other such as in Figure 5.24, it is possible to determine a point where the velocity is so low as to to produce zero p.e.

In order to do this, a straight line must be fit, and an appropriate number of points to fit over chosen. This can be done by calculating the $\chi^{2} /$ number of degrees of freedom for each possible combination of data points and selecting the solution closest to one. The top plots of Figure 5.25 show this, the results being used to produce the straight line fits shown below. The value of $\beta$ when the n.p.e. becomes zero is shown, and hence the corresponding minimum momenta of pions and muons required to fire the cerenkov detectors. The most significant point to note is that the values are not the same for the two counters.

Figure 5.26 shows that for a $1.8 \mathrm{GeV}$ muon sample, with a nominal spread, a cerenkov firing only on the muons above $1.77 \mathrm{GeV}$ detects a significant fraction less than the counter that is sensitive to muons down to $1.65 \mathrm{GeV}$. For this set of runs, the cerenkovs were set to their maximum pressure; it is thought that counter one 

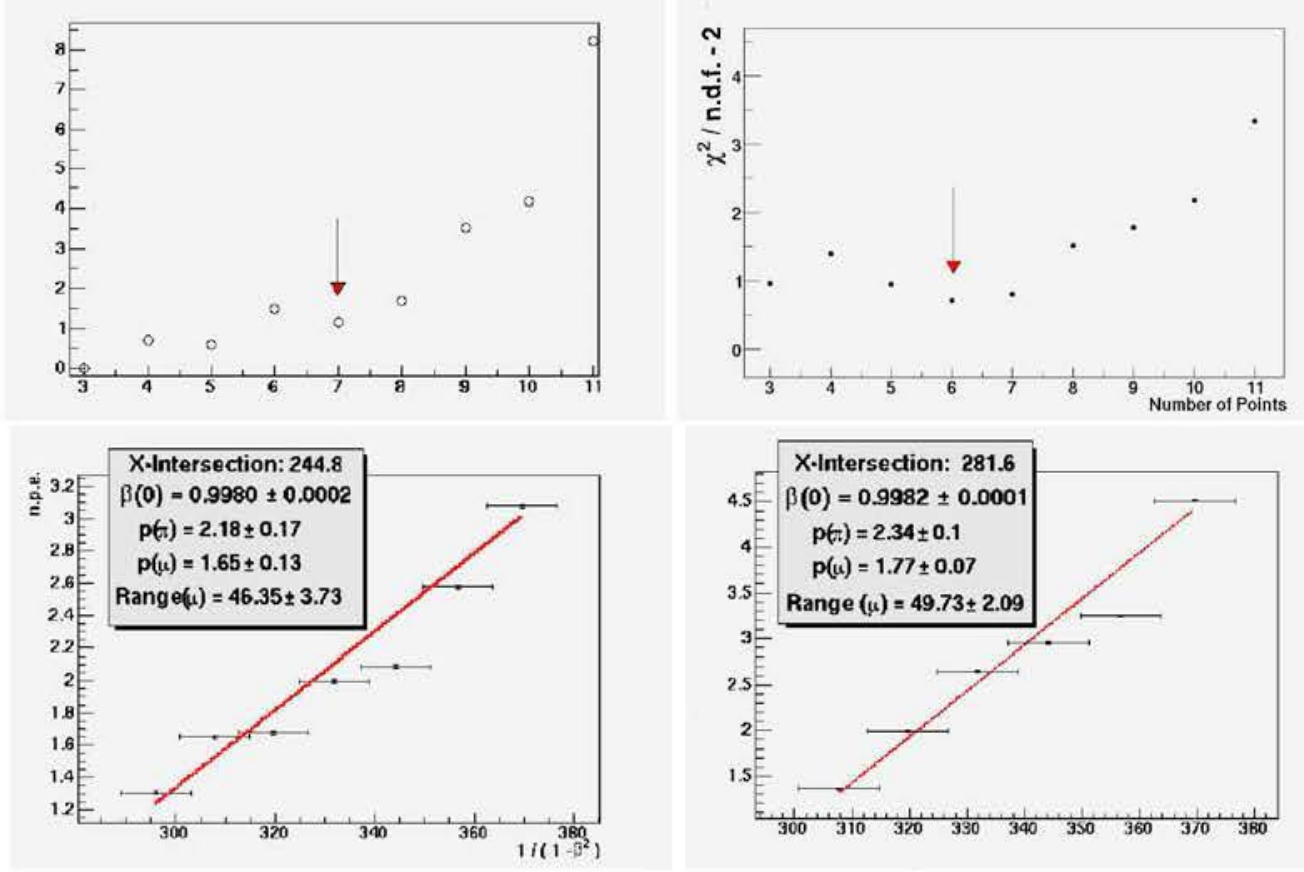

Fignese 5.25: The top plots show the $X^{2} /$ mmber af degrees of freedom for stratght line fits to the plots shown in Fume 5.24. Once the best mumber of points to be used was fomm, the bottom plots show the fit to these points. By calculating the $\mathrm{X}$-intercept of this line, the value of $1 / 1-\beta^{2}$ that corresponds to zero photoelectrons beimg produced can be fomd. Findlly, using the masses of pions and mmons, the effective threshol momenta to fire the cerenkovs can be calculated. For the upper and lower plots, the two on the left are for forenkoy 3 (upstrean) and the two an the right are for cerenkov 1 (downstream).

may have been pmoned slightly higher than cerenkey three, causing this effect.

The efficiencies can be corrected for this effect, the new result being shown in Figme 5.27. Whilst beimge improved, they still exhibit some stramge effects at low ramges. A small aditional comection can be made for the pions that decay to mums between the two cerenkoy commers, that may compensate for this. The following section demonstrates that this correction is small however, and is in fact overestimated by simulations.

Returmug to the plots of range detected in one, both or meither cerenkovs, (Figsme 5.28 ). the comection for efficiency is show in red, and the smmation of the datat 


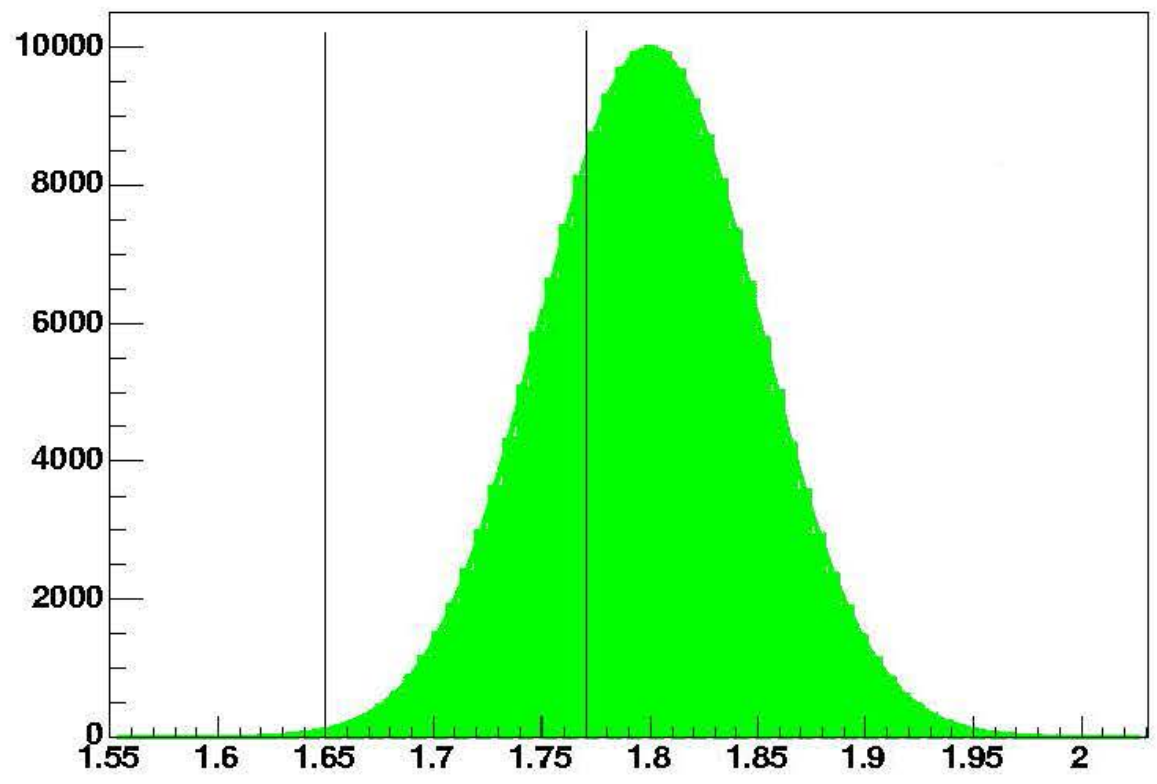

Figure 5.26: The distribution of muon momentat at $1.8 \mathrm{GeV}$, showing the 1.65 and 1.77 Gev muon culoff points. At a higher pressure, significantly nore mons are seen in one cerenkoy than anohlhes.
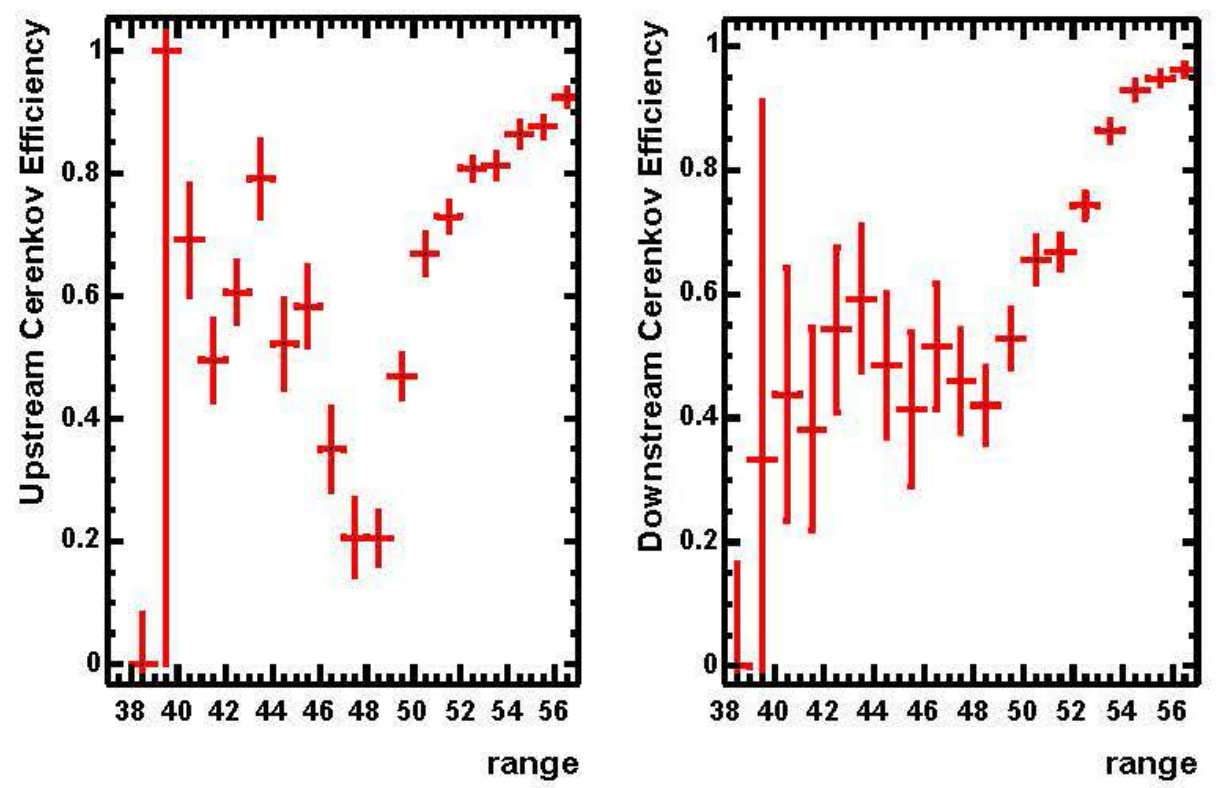

Figure 5.27: Corrected plane-ly-plane efficiencias. 
and correction is shown in black (Figure 5.29). Before, the counters were preferentially firing on the higher energy muons. Whilst this is still the case, the efficiency correction has had the effect smoothing out the muon distribution. The fit to the peak of the muons that fired both cerenkovs has moved back significantly compared to Figure 5.21, illustrating that fairer representation of the true muon spectrum present has been established.
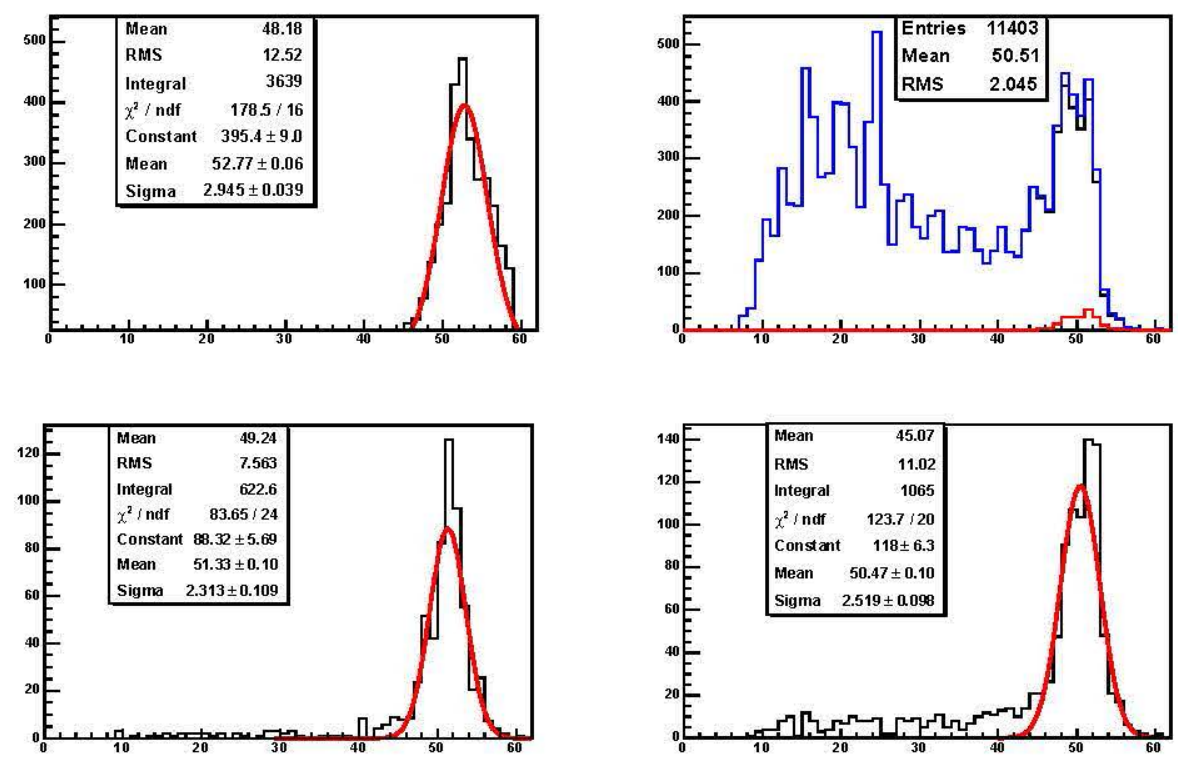

Figure 5.28: Fits to range plots. Top Left: Events that fired both cerenkov detectors. Top Right: Events that fired neither cerenkovs. Bottom Left: Events that fired only the first cerenkov. Bottom Right: Events that fired only the third cerenkov.

\subsection{Muon selection using shape and energy cuts}

\subsubsection{Muon Spectrum}

The muons in the CERN test beams are unlike the other particle species in that their spectrum is never mono-energetic. Whilst particles of the desired momenta are selected out using appropriate magnetic fields, the muons arise from decays of momentum selected pions. In the rest frame of the pion, the decay occurs isotropically but is then boosted to the lab frame, resulting in two momentum peaks; one 

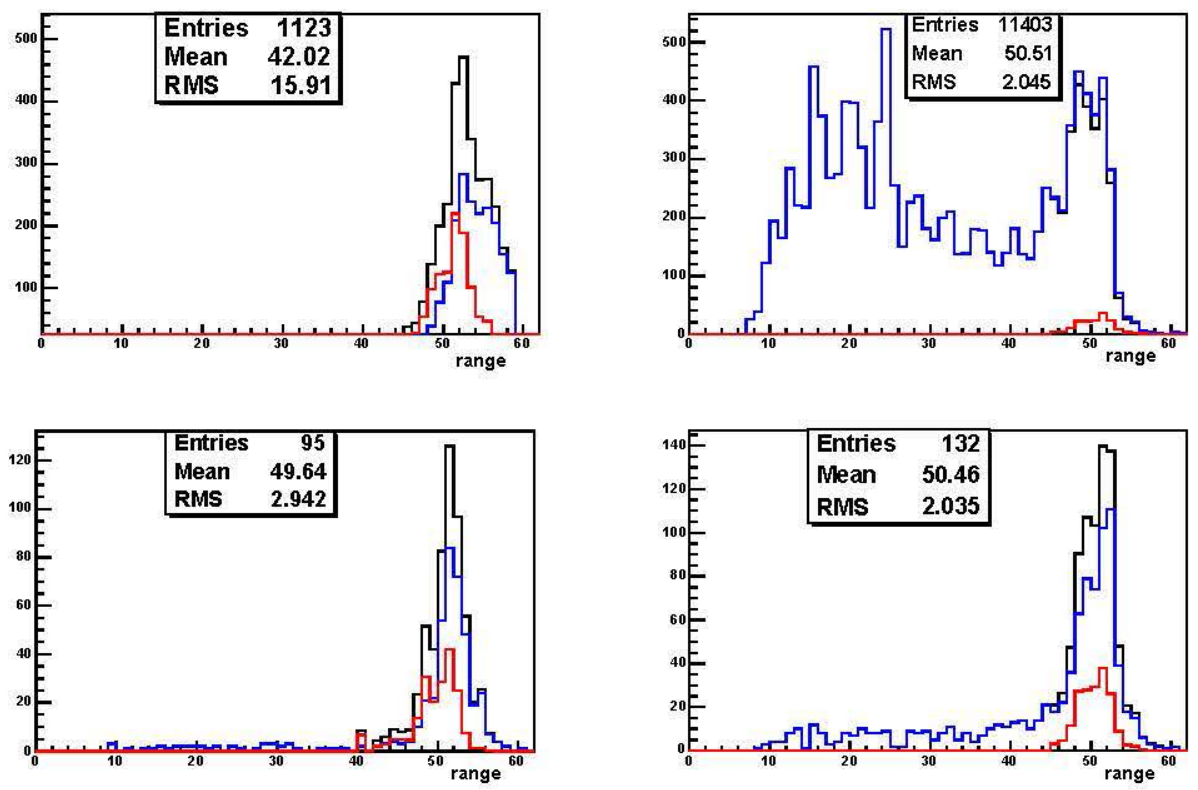

Figure 5.29: Corrected range plots. The original data is shown in blue, the correction in red and the final result in black. The description of the various plots is given in Figure 5.28

where the muon travels in the same direction as the decayed pion and the other in the opposite direction. The energy and momentum of the resultant muon are given by:

$$
\begin{aligned}
& E_{p}=\frac{m_{\pi}^{2}+m_{p}^{2}}{2 m_{\pi}} \\
& p_{p}=\frac{m_{\pi}^{2}-m_{p}^{2}}{2 m_{\pi}}
\end{aligned}
$$

Thus the maximum energy the muon can have is the same as that of the pion and the minimum, if it decays in the opposite direction to the pions motion is $0.57 \mathrm{E}_{\pi}$. The distribution of muon momenta between these two peaks is further suppressed by the fact that muons with significant transverse momenta are more likely to miss the TOF paddles and hence not initiate a recorded event in the detector. 


\subsubsection{Beamline Simulation}

The simulation of particles in the $\mathrm{T} 7$ and $\mathrm{T} 11$ beamlines has three phases. The target is modelled using the FLUKA03 code which then inputs to the Decay TURTLE program. This simulates the particles as they travel through the beam optics regions before entering the test beam hall and the detector. In the T7 and T11 beamlines this distance is 32 and $40 \mathrm{~m}$ respectively, after which they reach the first cerenkov detector. TURTLE is able to model pions decaying to muons in this region, which will be referred to as the 'upstream' region. The simulated beam is then passed to a GEANT3 based program which simulates energy loss and decays in the region just before the detector and the response of the detector itself to the beam. This region will be referred to as the 'downstream' area. A number of simulations of muons were generated at various momenta (See Figures 5.30, 5.31 and 5.31). The characteristic 'two-horned' shape is best observed around $1.8 \mathrm{GeV}$. Muons that pass all the way through the detector tend to collect at plane 60 in these plots. Some muons will inevitably multiply scatter, causing them to leave the detector early and hence have a greatly reduced range.

The difference between the 'old' GEANT3-only muon model and the new 'full beamline' simulation can be seen in Figure 5.33.

\subsubsection{Particle Definitions}

The T7 and T11 testbeams are a noisy environment. In order to ensure good data quality, some global cuts must be applied to all events to remove overlapping, out of time and incomplete events. This is achieved by imposing a fiducial cut: events must be fully contained within the detector. Any events which have hits in the outer 3 strips (excluding crosstalk) or in planes 58 or 59 are deemed to have failed the fiducial cut. The exception to this is PS muons which are actually selected by having failed the fiducial cut in the last 6 planes of the detector.

Events that are out of time may well be written to disk or subsequently reconstructed incompletely. The same applies to events that are seen in the detector but have not passed through the TOF paddles and set off the triggering sequence correctly. To remove this class of events from the data, there must be no scintillator 

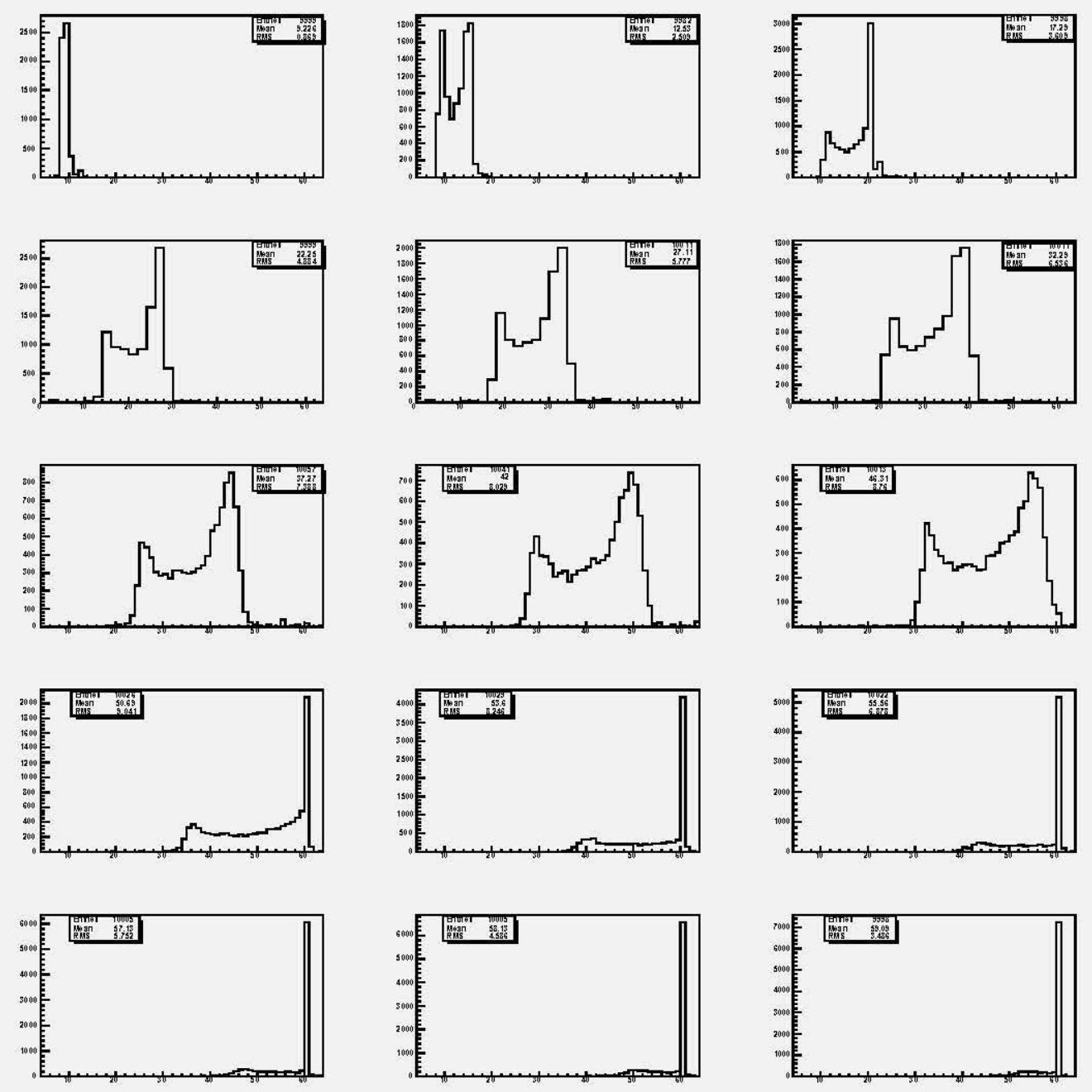

Figure 5.30: Showing the range of muons of various momenta in CalDet, according to the T11 Monte Carlo. The momenta simulated are $0.4,0.6,0.8,1.0,1.2,1.4,1.6$, $1.8,2.0,2.2,2.4,2.6,2.8,3.0$ and $3.2 \mathrm{GeV}$ from top left to bottom right. As might be expected, the mean range increases with momenta with peaks at 60 indicating muons that passed entirely through the detector. 

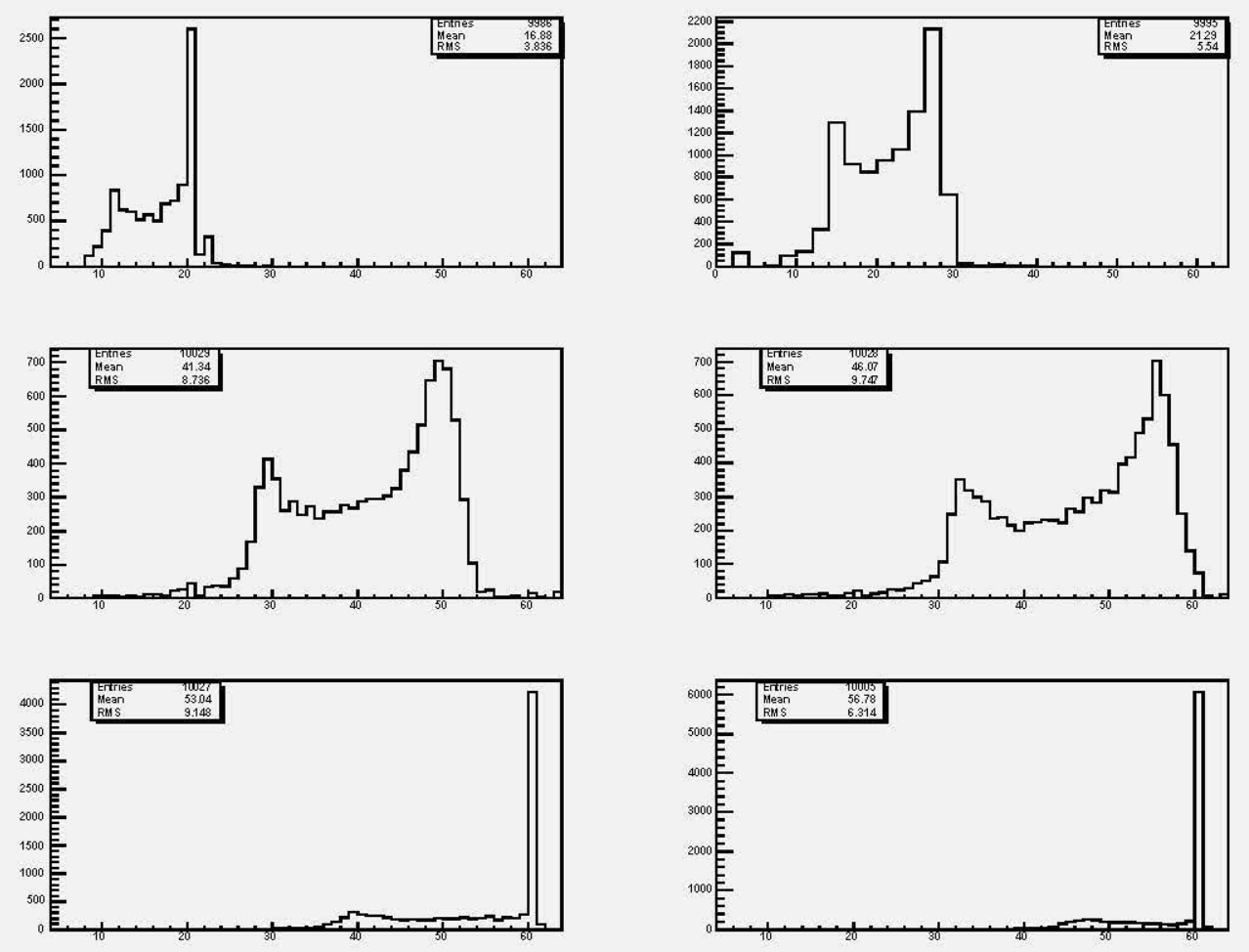

Figure 5.31: Showing the range of muons of various momenta in CalDet, according to the T7 Monte Carlo. The momenta simulated are $0.8,1.0,1.8,2.0,2.4$ and 2.8 $\mathrm{GeV}$. The peak at 60 indicated muons that passed entirely through the detector.

hits with a time that is less than 30 ns before or greater than $295 n$ after the TOF trigger time. Events that do not have hits in strips $9-12$ in the first 4 planes are also rejected. Finally, events that have not been fully reconstructed in both views are removed.

Once a clean data set has been established, particles must be separated by species. In the T7 and T11 beamlines, the TOF system, the cerenkov detectors and CalDet itself are used to achieve this.

- TOF:Particles are selected upstream by bending magnets to have the same momentum. Since the baseline between the two TOF paddles is a constant fixed distance, intrinsically heavier particles will have a lower velocity than lighter ones. This corresponds to a difference in time between the signals in the TOF paddles for heavy (i.e. protons) and light (i.e. electrons) particles 

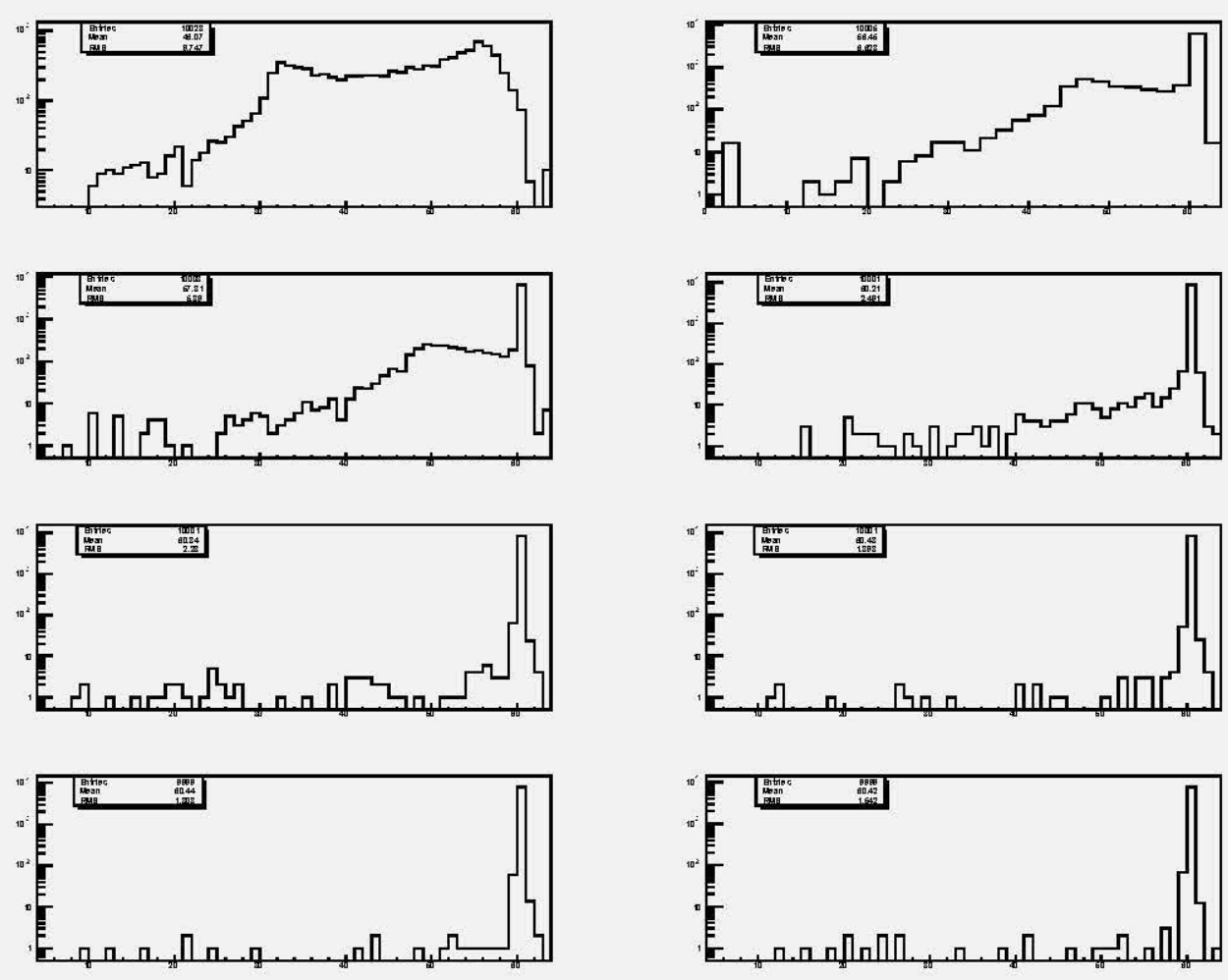

Figure 5.32: Showing the range of muons of various momenta in CalDet, according to the T7 Monte Carlo. The momenta simulated are $2.0,2.8,3.0,4.0,5.0,6.0,8.0$ and $10.0 \mathrm{GeV}$ from top left to bottom right. Note the log scale used, the first two plots are also shown on a linear scale for comparison in Fig. 5.31.

given by:

$$
\Delta t_{a b}=\frac{l}{c}\left(\sqrt{1+\frac{m_{s i}^{2}}{p^{2}}}-\sqrt{1+\frac{m_{b}^{2}}{p^{2}}}\right)
$$

where 1 is the separation of the TOF paddles, $c$ is the speed of light and $p$ is the beam momentum. $m_{a}$ and $m_{b}$ are the masses of two chosen particle species. Unfortunately, electrons, pions and muons have too similar masses to allow them to be accurately separated by their TOF time differences.

- Čerenkov:The Cerenkov counters also work on the principle of separating particle types by the speed at which they travel through them. In any medium, the local speed of light is lower than that in a vacuum. Particles travelling 


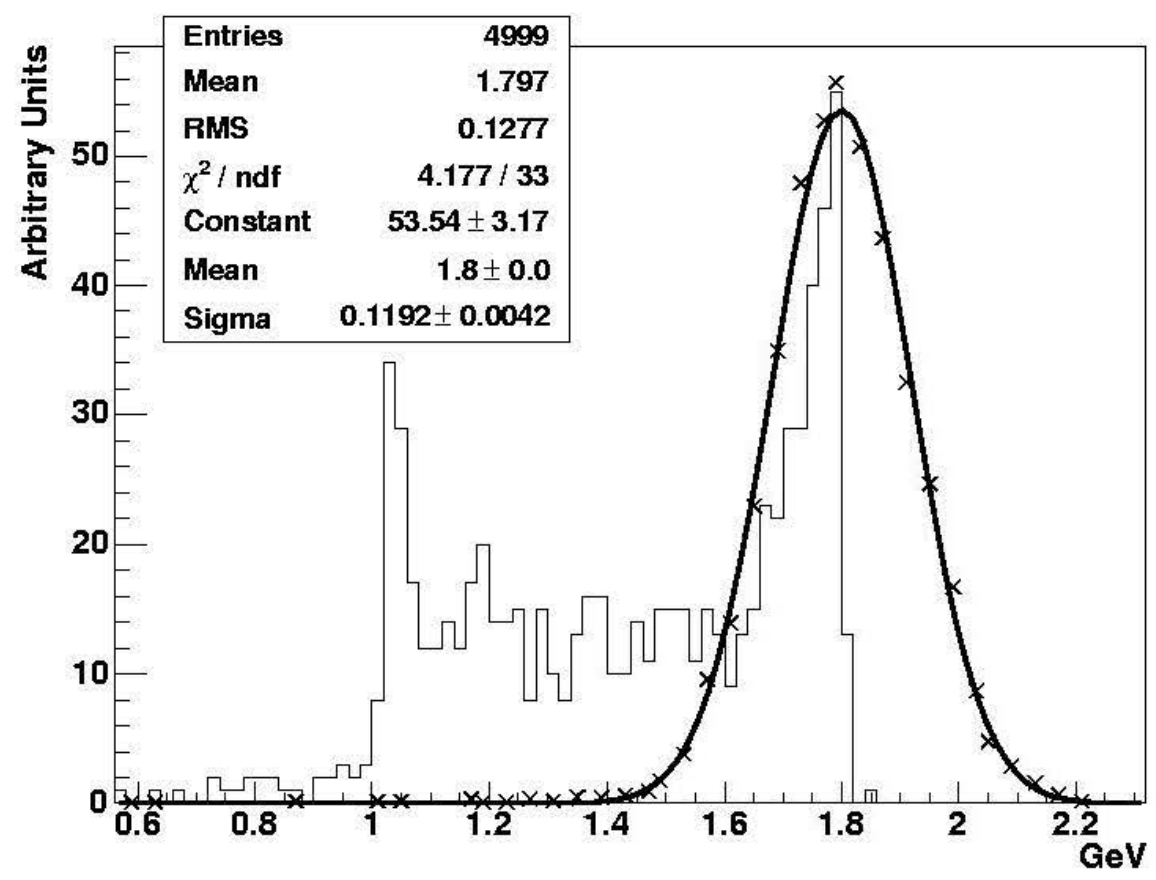

Figue 5.33: The plotied points show the momentum distribution for $1.8 \mathrm{GeV}$ muons with a $5 \%$ energy suread. The events were generated with GEANT3 and a gatssian fitted. The histogram shows a more realistic simulation where the muons that have arisen from decas of 1.8 GeV pions and have travelled down the $\mathrm{T} 7$ beamline.

h.hough a medium (such as carbon dioxide in the case of a Cerenkov connter) with sufficient veloctly catse photons to be emitted in a cone described by the formula:

$$
\theta_{t \in t e n t a n s}=\cos ^{-1}\left(\frac{1}{R(1+k P)}\right)
$$

where $P$ is the pressure in the Cerenkov comnter and $\mathrm{k}$ is $4.1 \times 10^{-4}$ for $\mathrm{Co}_{2}$. Electrons will typically always fire the connters, but by raising the pressure, increasingly heary paticles can be set to fue it.

- CalDet:CaDel's power in particle identification cones from the reconstumed shower shape of the event. Electrons interact electomagnedically after lithe penetation into CaDed producing short, wide showes consisting of many associated hits. Muons are minimum ionising particles and produce long, straight: 
tracks, typically consisting of one hit per plane in each view. Protons and pions interact hadronically in the detector, producing event shapes similar to electrons. Some pions drift through the detector without interaction however and have similar event shapes to muons.

Once the shower data has been reconstructed, two event quantities that can be calculated and have been found to have particular strength in separating muons and pions are known as 'multiplicity' and 'E3'. The multiplicity is calculated by summing the total number of hits in an event and dividing by the number of hit planes. For muons this is expected to be around 2 since the read-out is double ended in each plane. Pions which tend to shower will have a higher value. E3 is found by moving through all the planes that contain the event and summing the energy deposited in those planes. The highest three-plane-energy sum in the event is then recorded and divided by the total energy deposited in the entire event. Muons will tend to deposit most energy in the last three planes (see Section 5.7) but it will only be a modest fraction of the total event energy. Pions on the other hand can shower at any time and deposit a large fraction of their total energy in a three plane window. The distributions of these two cuts can be seen in Figure 5.35. It can be seen that these cuts are not strongly correlated with each other and so when used in parallel, provide excellent discriminating power between pions and muons. The results, when applied to Monte Carlo, are shown in Figure 5.36. The precise cut values are shown below. The cuts successfully identify $94 \%$ of the muons in the muon Monte Carlo dataset with just under 3\% incorrectly identified as pions. $97 \%$ of the pions are correctly identified in the pion Monte Carlo dataset, with $3 \%$ being assigned as muons.

Using these cuts together with the TOF and cerenkov counters, all of the major particle types in the test-beams can be separated. Positively and negatively charged particles cannot easily be distinguished by these means. The following cuts describe the criteria used to identify various particles.

- Proton: Fires neither cerenkov and is seen in the second TOF paddle between 460 and 560 ns after the trigger. 
- Deuteron: Fires neither cerenkov and is seen in the second TOF paddle between 700 and 800 ns after the trigger.

- PS muon: Leaves the back of the detector or the side past plane 54.

- Beam muon (below 2.4 GeV): Does not leave the detector, the event has a multiplicity value between 1.84 and 2.55 and an E3 value less than 0.3 .

- Pion:The event has a multiplicity value less than 1.84 or greater than 2.55 and an E3 value greater than 0.3 .

Beam muons above $2.4 \mathrm{GeV}$ leave the detector and cannot easily be distinguished from PS muons on an event-by-event basis.

Having established a muon sample using the E3 and multiplicity cuts, it is interesting to compare the result to the sample selected by the cerenkovs. Figure 5.38 shows the expected contributions of muons from the upstream region. The plot shows predicted pion decay vertices, that is the point along the beamline that a pion decays to a muon. Events in bin 0 are muons that have been passed to GEANT by the TURTLE simulation, and the remainder are decays that occur further downstream. It can be seen that only around $14 \%$ of muons are thought to have been generated upstream, this is because many are off momentum or have some transverse component of their momentum that causes them to miss the TOF counters and fail to trigger an event. This is not in agreement with the observations from Figure 5.39 that show the fraction of muons observed in both cerenkovs compared to all muons seen, is much higher. Any muon that fired the upstream cerenkov must have been produced upstream. We see that around $32 \%$ of muons have done so, considerably more than the expected value of $14 \%$.

It is thought that the additional muons are created by a process referred to as 'scraping'. Collimators are used in the beamline to set the momentum spread of the particles in the beam and to vary the intensity. However, as the aperture of the collimators is decreased, the numbers of particles hitting them increases. It it thought that, contrary to the TURTLE decay simulation, these particles interacted with the collimators producing secondary showers upstream, of which muons are a significant component. This, then is the source of the additional muons seen. To 


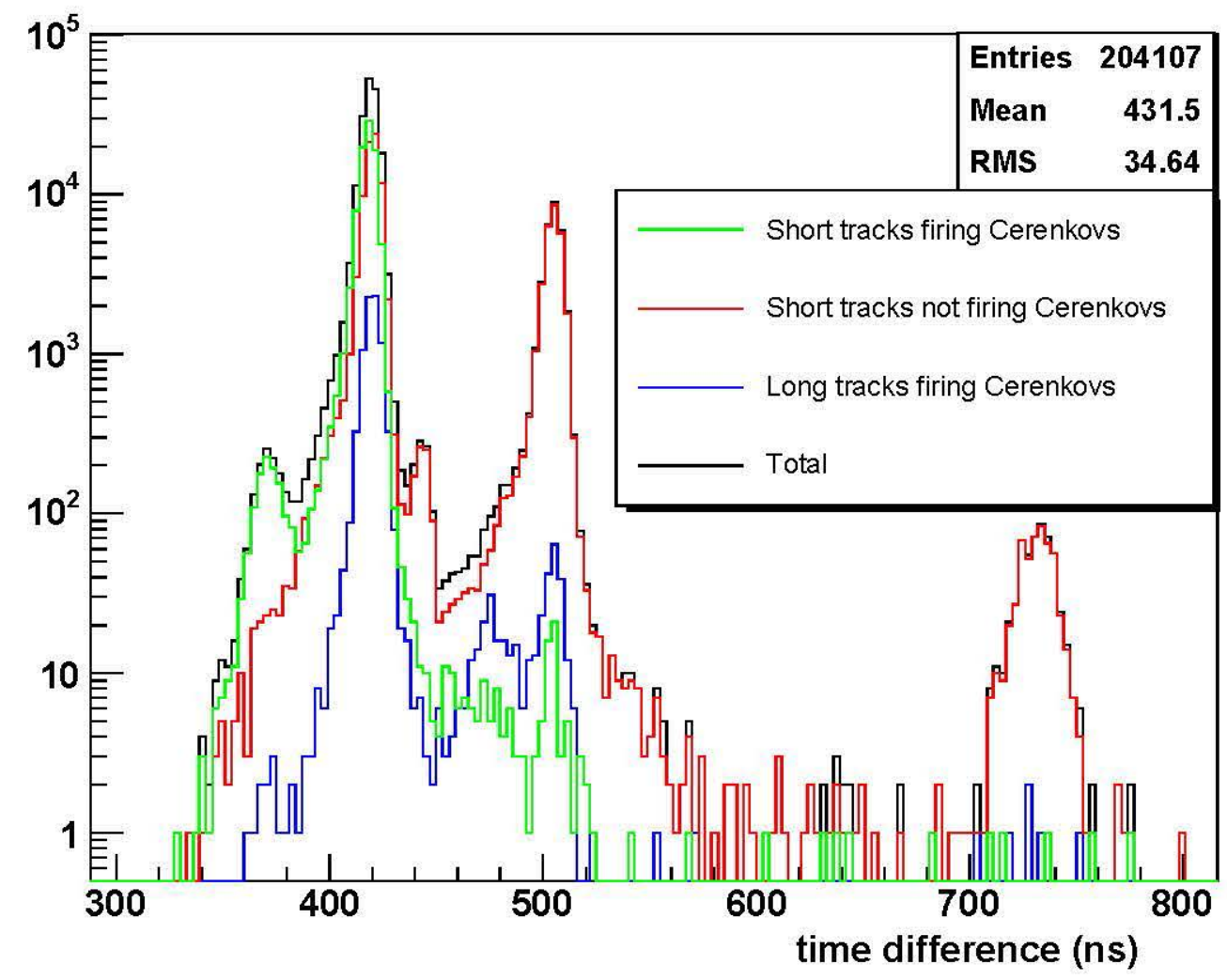

Figure 5.34: Showing the Time-of-Flight time differences between the two paddles in front of the detectors in the $\mathrm{T} 7$ beamline. Heavier particles travel more slowly for a given momentum producing a correspondingly larger TOF time difference. For this particular čerenkov pressure setting, particles with long tracks that fire the cerenkovs are muons, short tracked particles that fire the cerenkovs are electrons and short tracked particles that do not fire the cerenkovs are pions (320-460ns window), protons (460-540ns window) and deuterons (700-750ns window).

test this hypothesis, the beamline simulation was modified. First, the modelling prodecedure was broken down into three stages, and each considered separately. These stages were: the muons created in the upstream region of the beamline, the muons created in the downstream region of the beamline and pions that passed the muon cuts. These would be subtracted later. The relative ranges of these components are illustrated in Figure 5.40. Root's TFractionFitter routine was then applied to these three simulation components to find the best fit to data, for various collimator settings. The results are shown in Tables 5.3 and 5.4. In the tables, 

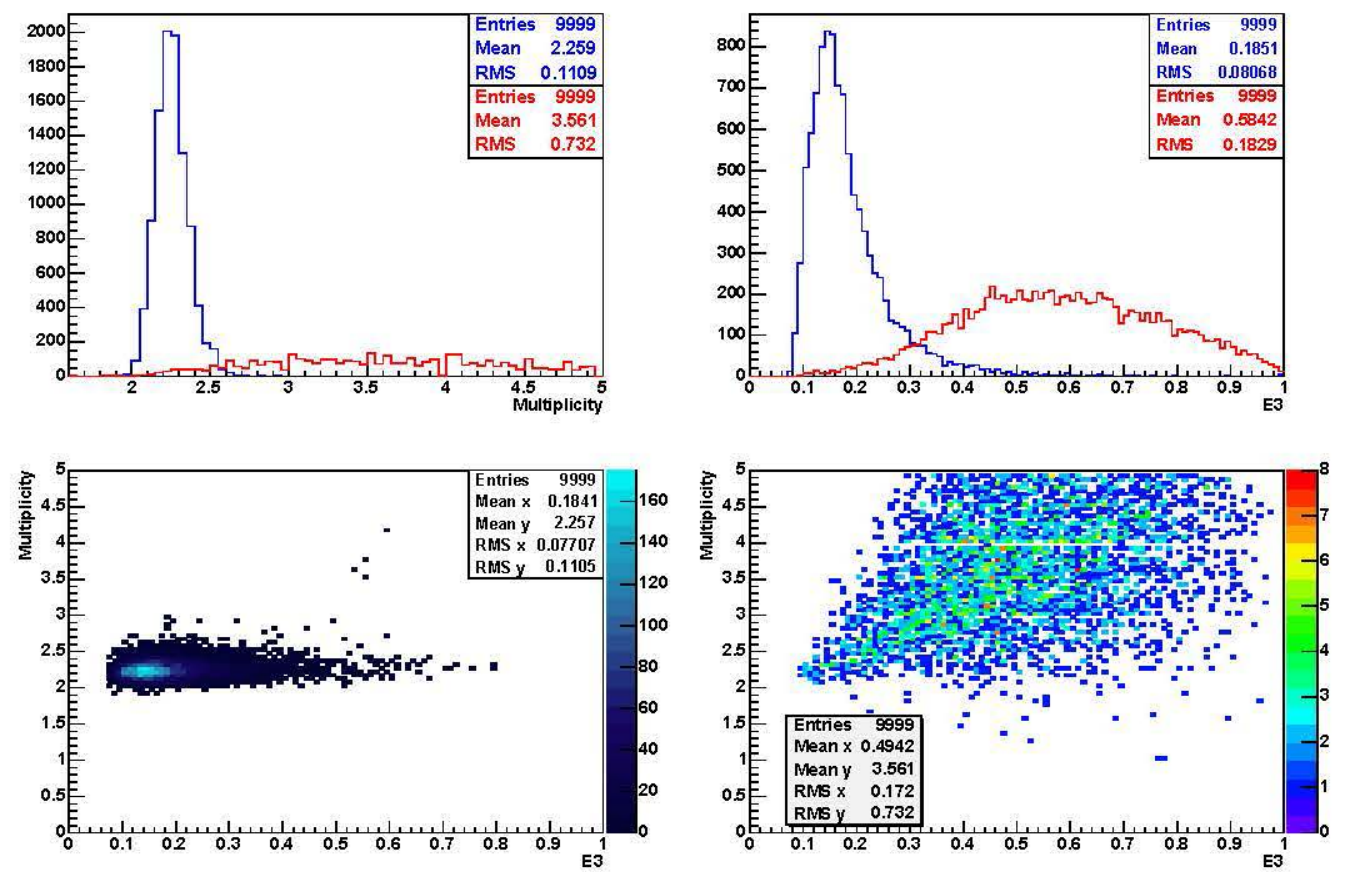

Figure 5.35: Figure showing the multiplicity and E3 cut distributions. Top left and top right show the total distributions with muons in blue and pions in red. Bottom left shows E3 against multiplicity for muons whilst the bottom right shows multiplicity against E3 for pions. Whilst there is some overlap of the pion distribution into the muon distribution, it can be seen that majority of the pion parameter space can be excluded owing to their wider, shower like nature.

MCH01 and MCV01 are the more upstream collimators, primarily concerned with the intensity control of the beam. MCH02 is in a region of higher dispersion in the beamline and is used to tune the momentum spread of the particles. 

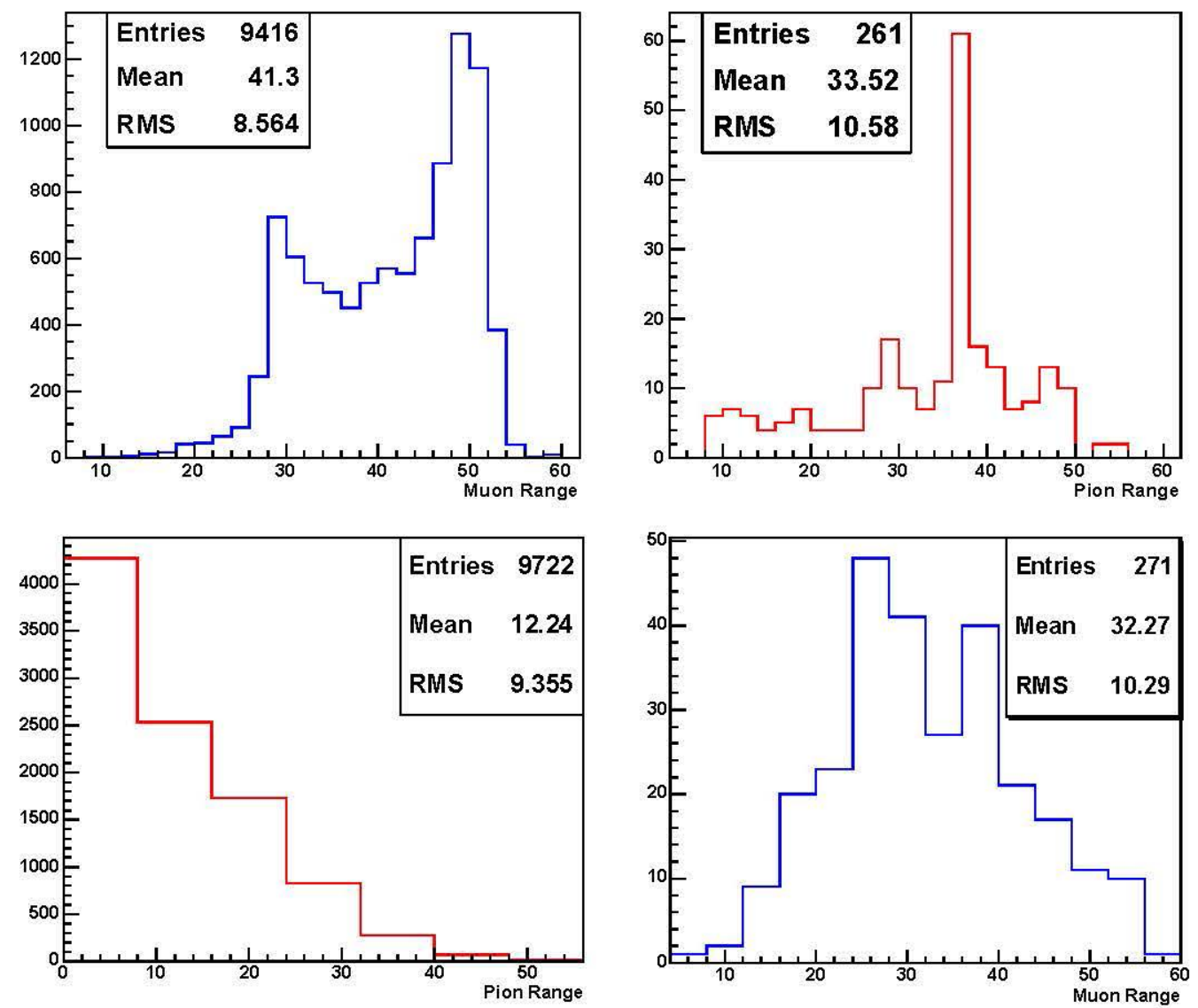

Figure 5.36: Showing the selection efficiencies for muons and pions. A sample of $1.8 \mathrm{GeV}$ Monte Carlo was generated for pions and muons (10,000 of each). The top left plot shows that the muon selection efficiency is $94 \%$ with $3 \%$ being incorrectly identified as pions (top right). The bottom left plot shows that the pion selection efficiency is $97 \%$ with $3 \%$ being incorrectly identified as muons (bottom right).

Table 5.5 shows the $\chi^{2}$ 's of various attempts at muon Monte Carlo simulation. The simplest form, monoenergetic muons with some energy spread does not describe the low energy tail of the muon distribution at all. The old beamline simulation was a great improvement but did not take scraping into account. When the facility to vary the fraction of muons coming from the upstream and downstream regions was added, the simulation began to accurately describe the observed data. The final subtraction of pions that passed the muon cuts gives the best fit.

Although in principle it would seem that a simple solution to the added complex- 

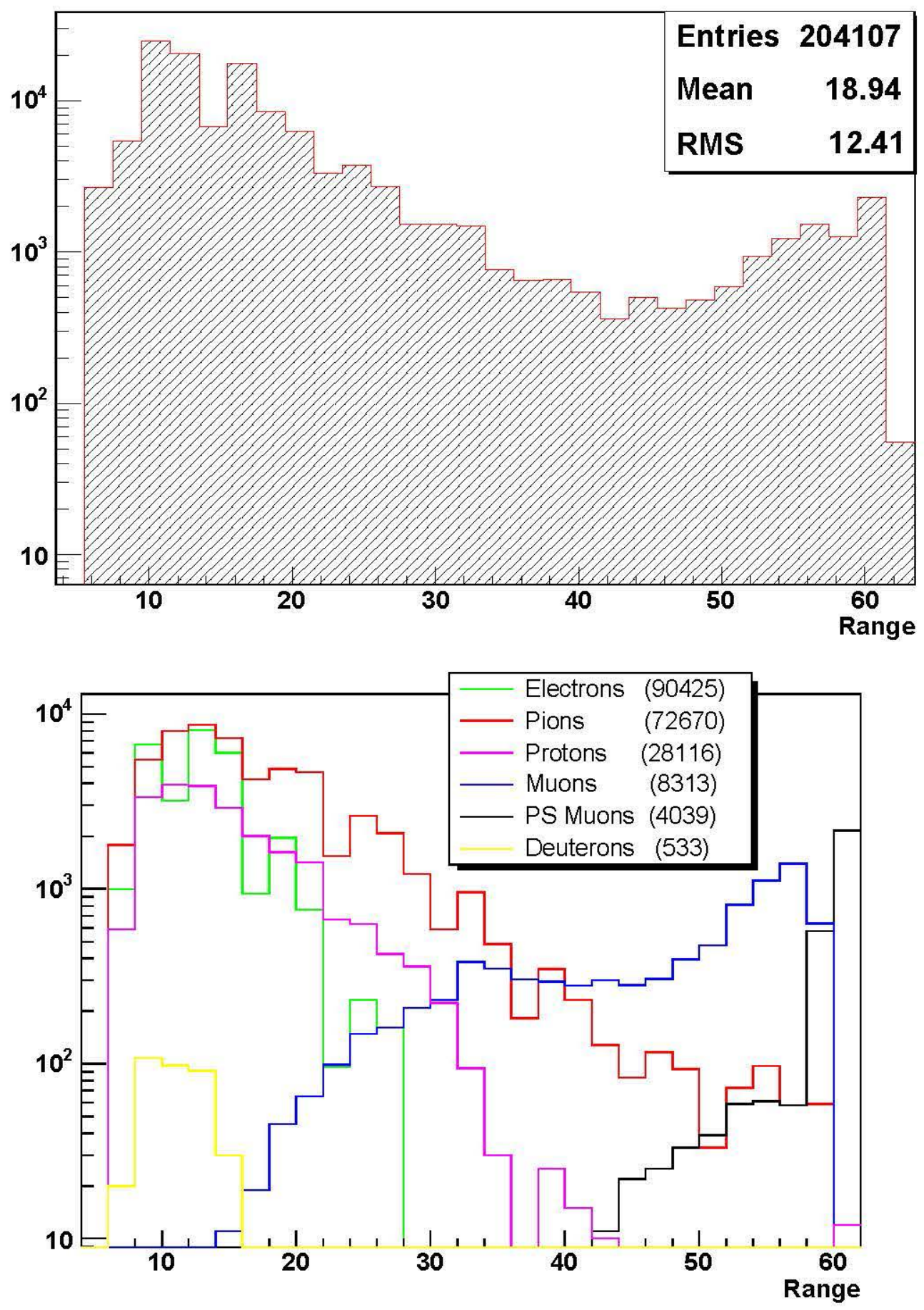

Figure 5.37: The top plot shows the ranges of all the particles seen in the detector. By using the cuts described, it is possible to decompose this into individual particle species (bottom). 

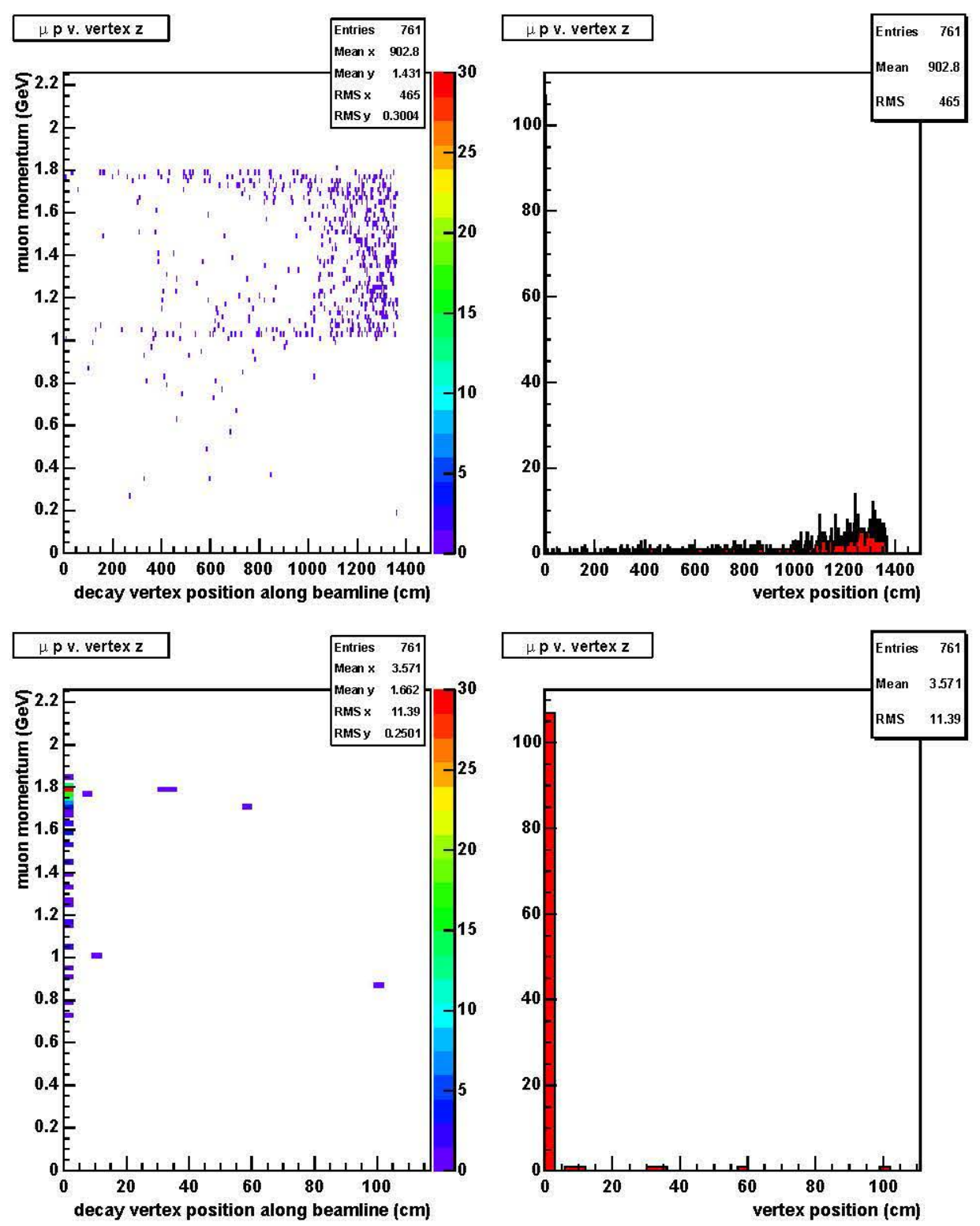

Figure 5.38: Figures showing the decay vertices of pions to muons in the GEANT3 simulation. The top left plot shows the distribution of resultant muon momenta and at what point along the beamline they decayed. The top right plot is a histogram of all these events, regardless of the final muon momenta. The muons that have been passed to this simulation from TURTLE are shown in the first bin. This is shown more clearly in the zoomed plots below, which focus on the start of the beamline. 107 muons (bin 1) from a total sample size of 761 show that around $14 \%$ of muons seen in CalDet are thought to have arisen from decays upstream. 


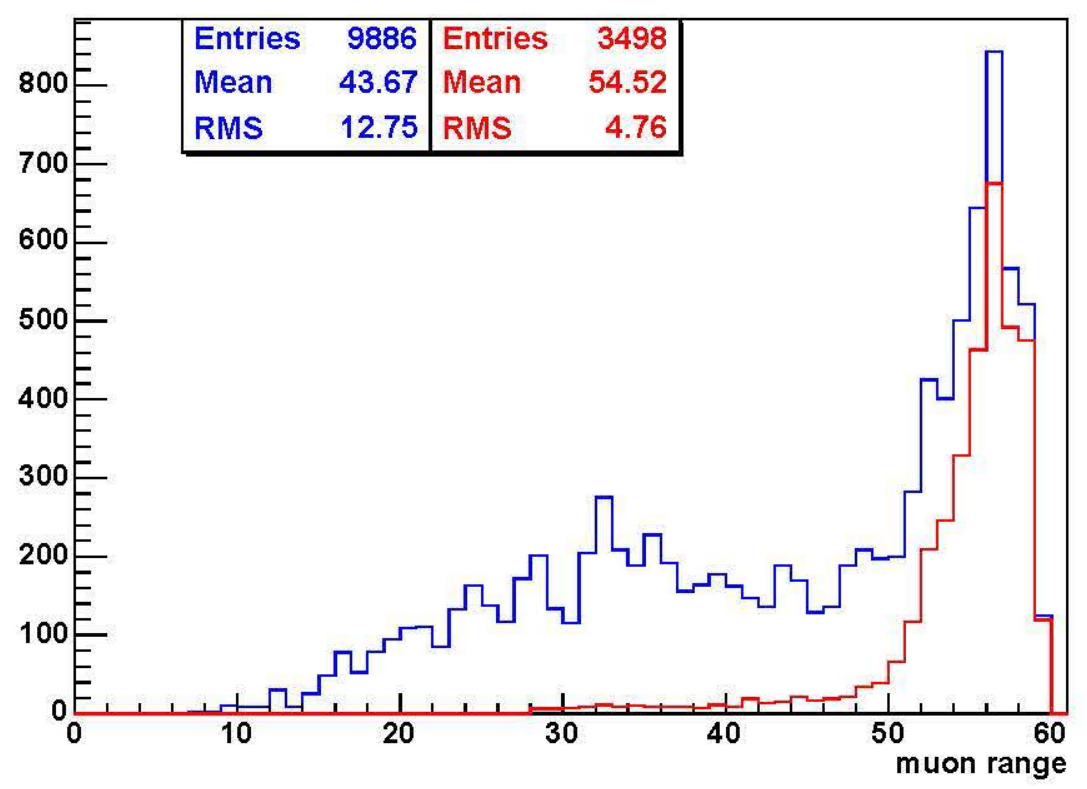

Figure 5.39: Showing (in dark blue) all muons selected using the shape/energy cuts previously described. In red are the muons that have fired both cerenkovs and have therefore necessarily been produced in the upstream region of the beamline. This fraction is $32 \%$, compared to $14 \%$ expected from simulation.

\begin{tabular}{|c|c|c|c|c|c|}
\hline Run Number & MCH01 & MCV01 & MCH02 & Up Fraction & Down Fraction \\
\hline 70799 & 3.5 & 3.5 & 2.6 & $0.61 \pm 0.01$ & $0.39 \pm 0.01$ \\
70830 & 3.8 & 4.1 & 2.6 & $0.60 \pm 0.01$ & $0.40 \pm 0.01$ \\
71521 & 5.8 & 5.5 & 2.4 & $0.33 \pm 0.02$ & $0.67 \pm 0.02$ \\
70796 & 5.5 & 5.9 & 2.5 & $0.35 \pm 0.01$ & $0.65 \pm 0.02$ \\
$70924^{*}$ & 6.0 & 6.2 & 2.3 & $0.29 \pm 0.01$ & $0.71 \pm 0.02$ \\
71266 & 6.7 & 6.7 & 2.3 & $0.33 \pm 0.01$ & $0.67 \pm 0.02$ \\
70570 & 6.9 & 7.1 & 2.5 & $0.40 \pm 0.02$ & $0.60 \pm 0.02$ \\
70802 & 7.3 & 7.3 & 2.6 & $0.34 \pm 0.01$ & $0.66 \pm 0.02$ \\
\hline
\end{tabular}

Table 5.3: Muon Energy is $+1.8 \mathrm{GeV}$. All runs are from $\mathrm{T} 72003$, target number is 2 . Order of elements is: Target, MCH01, MCV01, MCH02, CalDet. *runlog comment: BHZ01 off. 


\begin{tabular}{|c|c|c|c|c|c|c|c|}
\hline Run Number & Energy & Target & MCH01 & MCV01 & MCH02 & Up Fraction & Down Fraction \\
\hline 70712 & -1.8 & 2 & 2.5 & 7.0 & 2.5 & $0.42 \pm 0.01$ & $0.58 \pm 0.01$ \\
70703 & -1.8 & 2 & 4.0 & 7.0 & 2.5 & $0.42 \pm 0.02$ & $0.58 \pm 0.02$ \\
70707 & -1.8 & 2 & 4.0 & 7.0 & 2.5 & $0.42 \pm 0.01$ & $0.58 \pm 0.02$ \\
70466 & -1.8 & 2 & 6.8 & 4.0 & 2.5 & $0.41 \pm 0.01$ & $0.59 \pm 0.02$ \\
70574 & -1.8 & 2 & 6.9 & 7.1 & 2.5 & $0.40 \pm 0.02$ & $0.60 \pm 0.02$ \\
\hline 70402 & +2.0 & 3 & 2.9 & 2.6 & 3.0 & $0.62 \pm 0.01$ & $0.38 \pm 0.01$ \\
70730 & +2.0 & 2 & 4.0 & 3.8 & 2.5 & $0.46 \pm 0.01$ & $0.54 \pm 0.01$ \\
71267 & +2.0 & 2 & 6.7 & 6.7 & 2.3 & $0.33 \pm 0.01$ & $0.67 \pm 0.02$ \\
70525 & +2.0 & 3 & 6.9 & 7.1 & 2.5 & $0.40 \pm 0.02$ & $0.60 \pm 0.02$ \\
\hline 70405 & -2.0 & 3 & 2.9 & 2.6 & 3.0 & $0.62 \pm 0.02$ & $0.38 \pm 0.02$ \\
70516 & -2.0 & 3 & 12.6 & 12.8 & 2.5 & $0.30 \pm 0.02$ & $0.70 \pm 0.02$ \\
70518 & -2.0 & 3 & 12.6 & 12.8 & 2.5 & $0.30 \pm 0.02$ & $0.70 \pm 0.02$ \\
70521 & -2.0 & 3 & 6.9 & 7.1 & 2.5 & $0.40 \pm 0.01$ & $0.60 \pm 0.02$ \\
70809 & -2.0 & 2 & 4.3 & 4.3 & 2.6 & $0.51 \pm 0.02$ & $0.49 \pm 0.02$ \\
71362 & -2.0 & 2 & 6.4 & 6.6 & 2.4 & $0.34 \pm 0.01$ & $0.36 \pm 0.02$ \\
\hline
\end{tabular}

number is 2. Order of elements is: Target, MCH01, MCV01, MCH02, CalDet. 

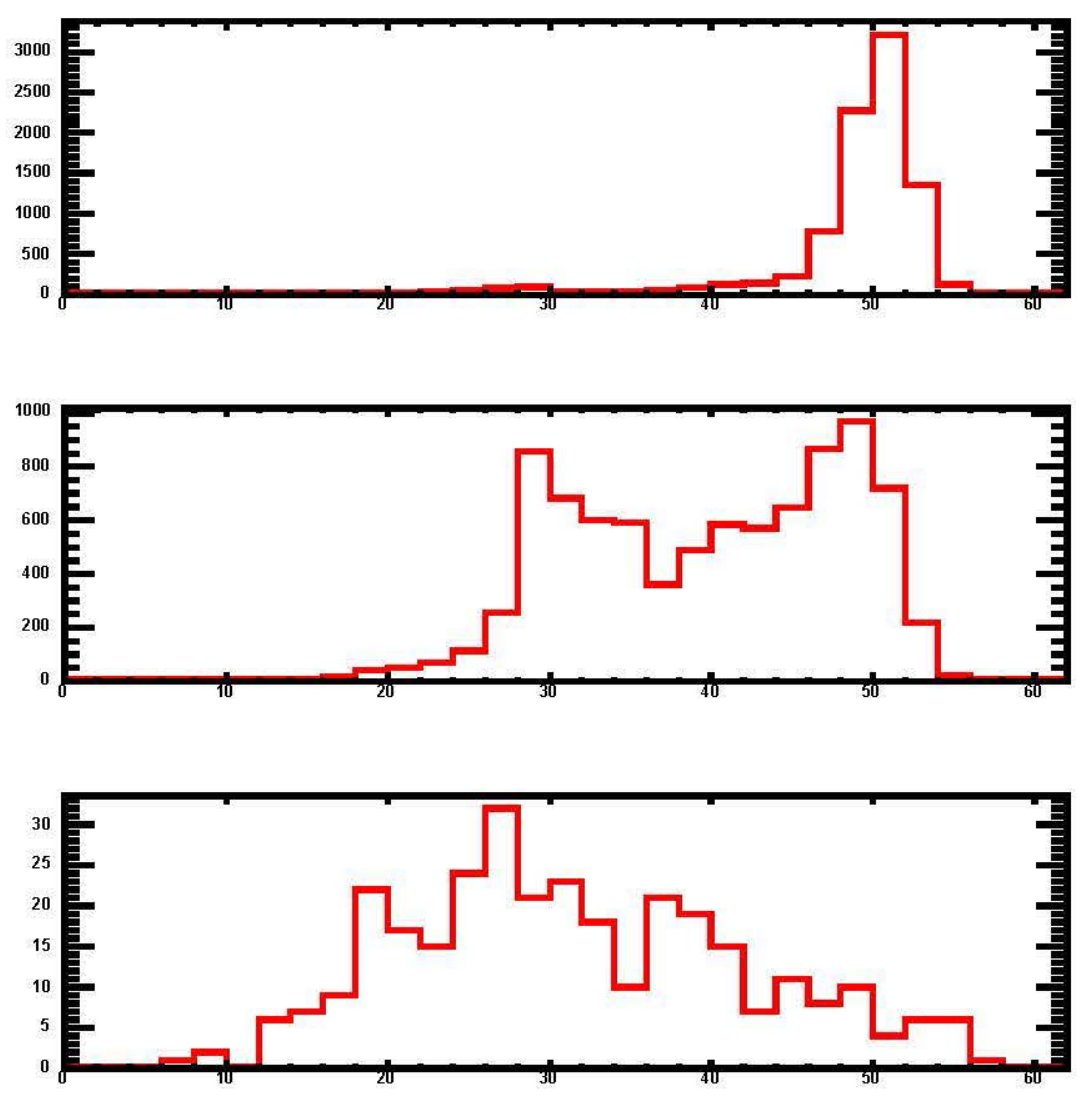

Figure 5.40: To obtain the most accurate Monte Carlo representation of the muons seen in the test beams at CERN, three components must be considered. These are the muons created in the upstream region of the beamline (top), the downstream region (middle) and the pions that pass the muon cuts (bottom), which must be removed. The particles are characterised here by their ranges (x-axis).

\begin{tabular}{|c|c|c|c|}
\hline Monte Carlo & $\chi^{2}$ & N.D.F. & $\chi^{2} /$ N.D.F. \\
\hline Mono-energetic (5\% spread) & 4064.51 & 30 & 135.484 \\
Old beamline simulation & 2032.30 & 30 & 67.74 \\
Variable fraction simulation (no $\pi$ 's) & 693.27 & 29 & 23.91 \\
Variable fraction simulation ( $\pi$ 's subtracted) & 64.429 & 28 & 2.30 \\
\hline
\end{tabular}

Table 5.5: Showing how well each version of the muon Monte Carlo fit the data. 


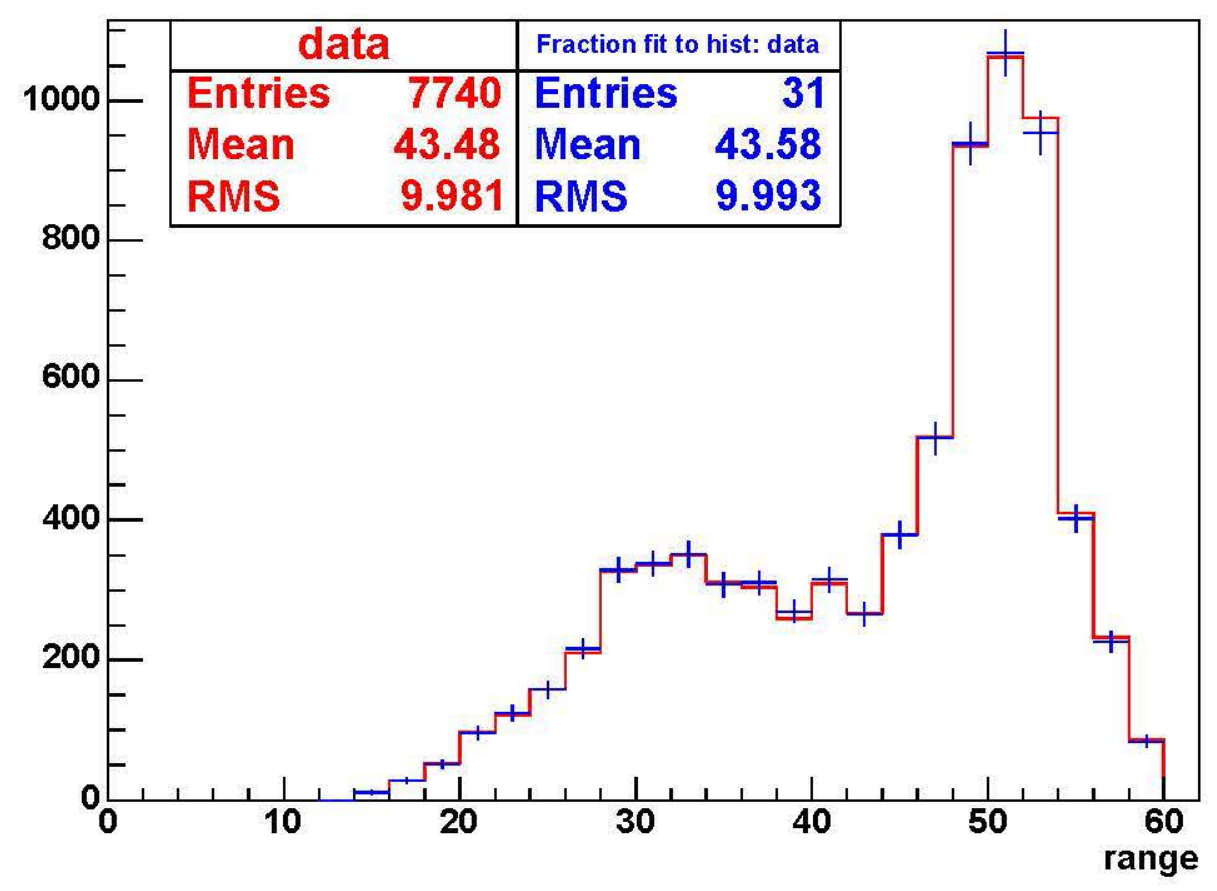

Figure 5.41: Showing the results of the fraction fitting procedure of beamline simulation elements to the data. At the largest collimator setting there is little upstream scraping and the profile of the muons is close to that predicted by the original simulation.

ity caused by the collimators would be simply to open them as wide as possible, in practice this is unfeasible. The collimators control the intensity of the beam; if set to high, the number of overlapping events seen in the detector increases. In general, all overlapping events have to be discarded since their energy measurement becomes uncertain.

The final problem to address is the question of how accurately the beam momentum was measured. Using the muon Monte Carlo with corrected upstream and downstream fractional components, various shifts in energy were then applied. This corresponds to small variations in the distribution to the left and right. The data was then fit to these modified distributions. Figure 5.43 shows the results. The resolution on this measurement is somewhat limited by the available binning of the modified distributions but it can clearly be seen that 1 (corresponding to the unmodified distribution) is not the best fit. Taking the root of the parabolic fit, a 


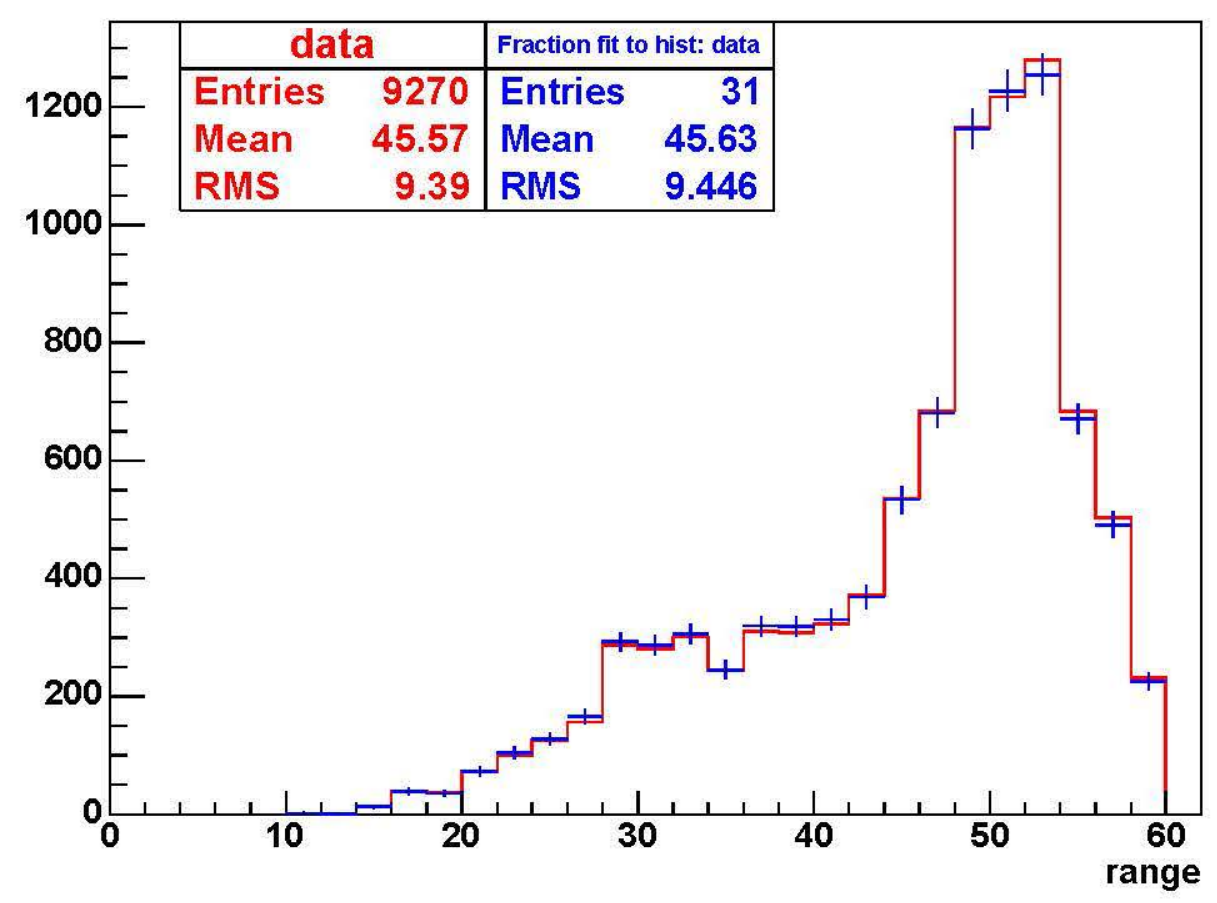

Figure 5.42: Showiing the results of the fraction fitting procedure of beamline simulation elements to the data. At the smallest collimator setting there is a large amount of scraping upstream, causing the ratio of on-momentum muons to increase.

value of around 1.028 is arrived at which corresponds to a muon energy of $1.85 \mathrm{GeV}$. From this simple procedure we find that the resolution on the measurement of the momentum of beam muons is around $3 \%$.

\subsection{Measurement of Muon $d \mathrm{E} / d \mathbf{X}$}

Muons travelling through the detector deposit energy in a manner described by the Bethe-Bloch formula. Figure 5.44 shows the theoretical characteristic BetheBloch form for muons travelling through copper ${ }^{4}$. The energy loss occurs as a result of ionisation of the medium being traversed; in physical terms, this is the electromagnetic scattering of the incident particle with the atomic electrons. The Bethe-Bloch curve has three regions of special interest:

- Low energy region: Here the rate of energy loss increases rapidly as $\beta^{-2}$.

\footnotetext{
${ }^{4}$ When minimum ionising, the difference in energy loss between steel and copper is around $3 \%$.
} 


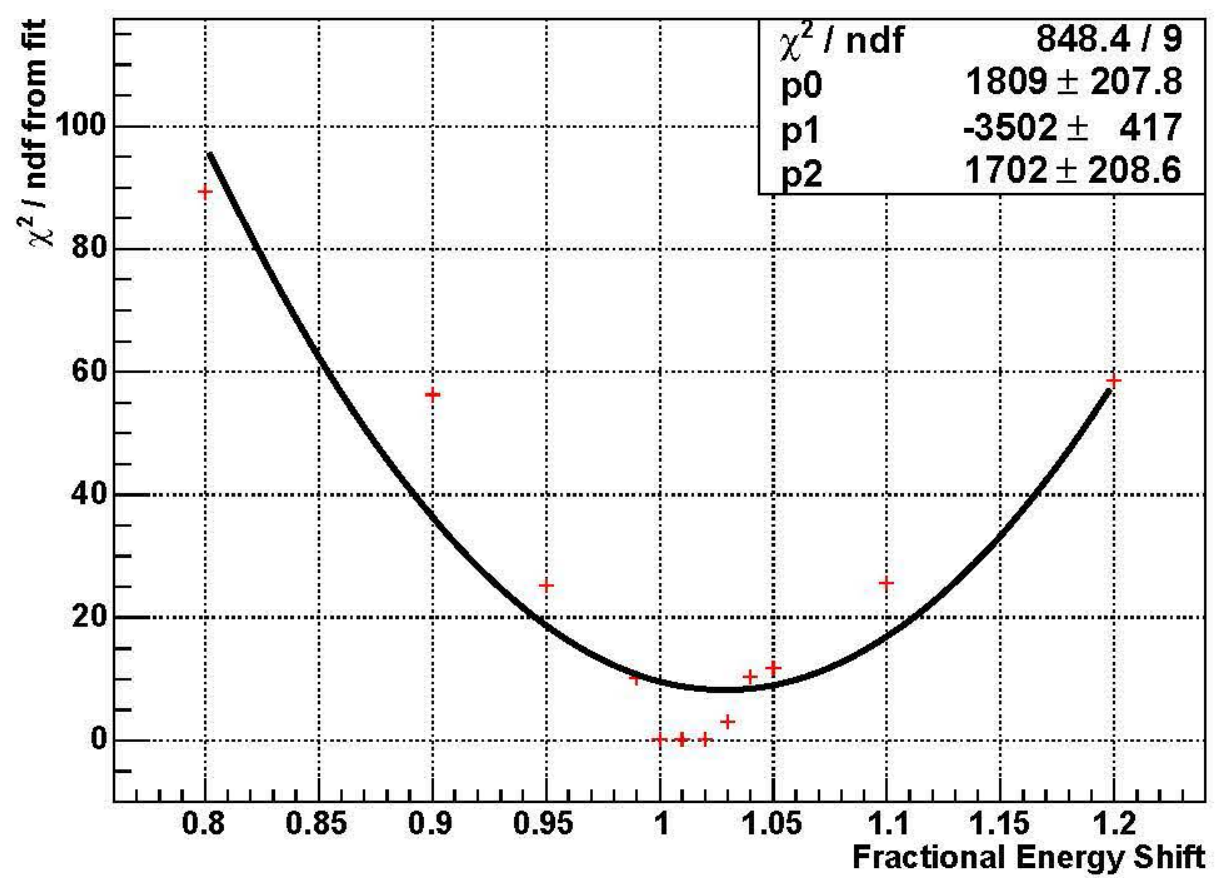

Figure 5.43: Showing the $\chi^{2} /$ n.d.f for various fractional energy shifts, indicative of the resolution on the beam momentum. The best fit does not appear to be at the expected value of 1 but at 1.028 which corresponds to a muon energy of $1.85 \mathrm{GeV}$.

- High energy region: The rate of energy loss increases but more slowly, as $\ln (\beta \gamma)$. This is known as the relativistic rise.

- Minimum Ionising region: When $\beta$ is around 0.96 , the travelling muon deposits the least energy.

Ionisation is the chief form of energy loss for muons with modest energies; Table 5.6 shows the quantity of energy lost by other mechanisms, in iron. Table 5.7 shows the corresponding losses in scintillator. The planes of the MINOS detectors are made from sheets of steel, sandwiched together with strips of scintillator. The scintillator is covered by a thin layer of aluminium for protection and light-sealing. An air gap separates each plane. Table 5.8 shows the relative energy loss expected to occur in each of the detector components.

Individual muons seen in the MINOS detector, deposit a wide range of energies on a strip-to-strip basis. This is because the loss itself in the scintillator follows a Landau distribution which is then convolved with a Gaussian distribution resulting 


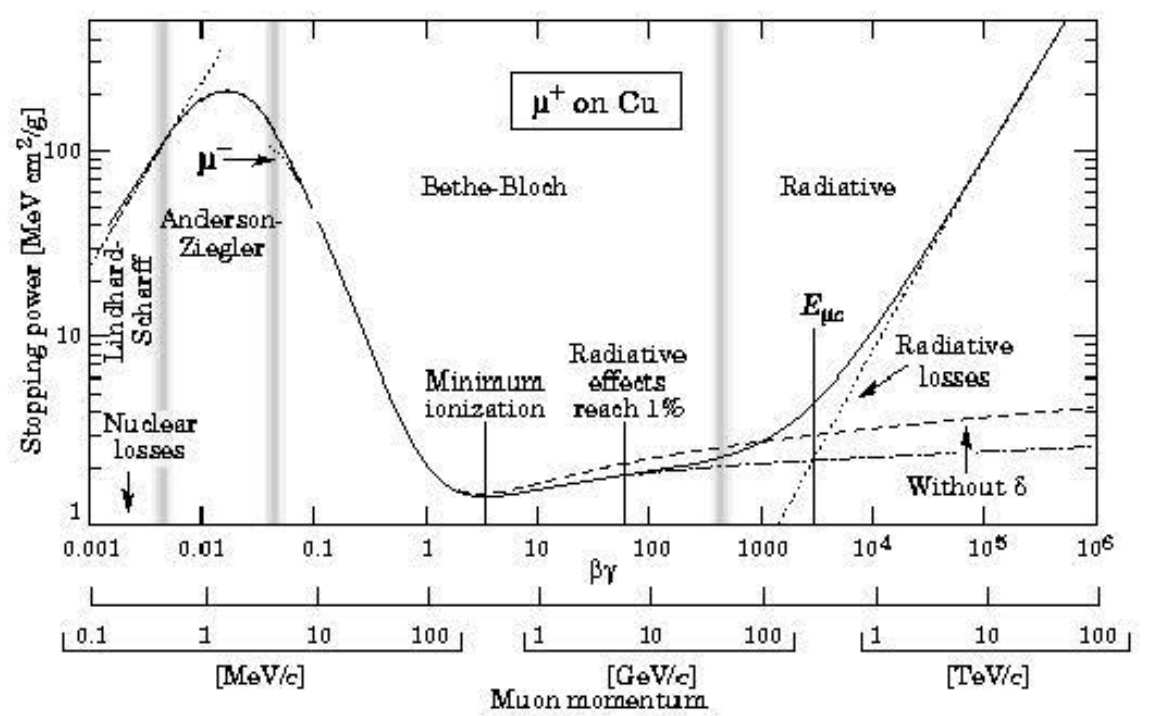

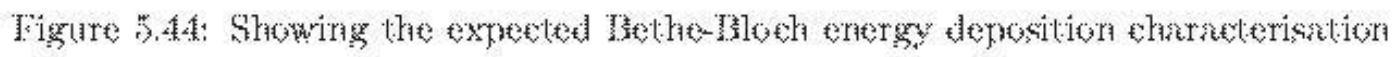

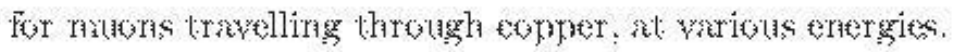

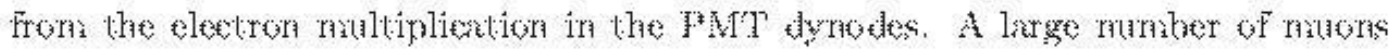

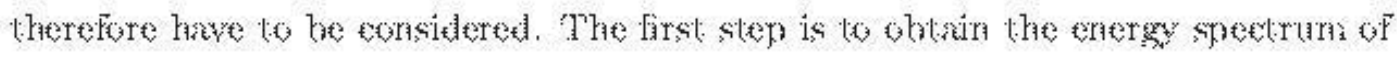

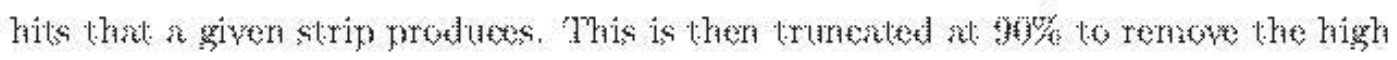

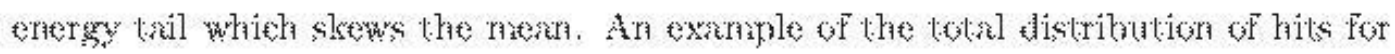

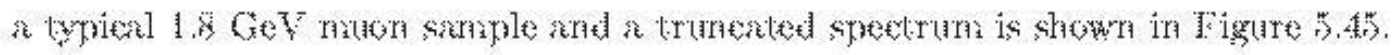

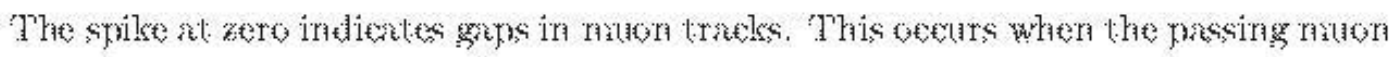
toes rot: protues erough light: ir the seirtillator or the ForT.

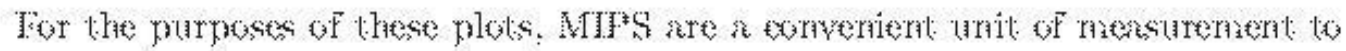

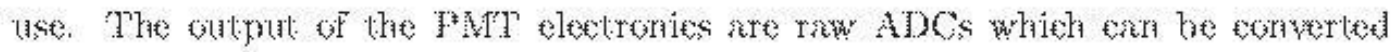

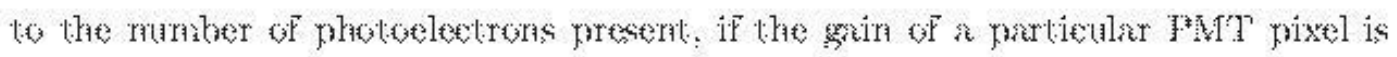

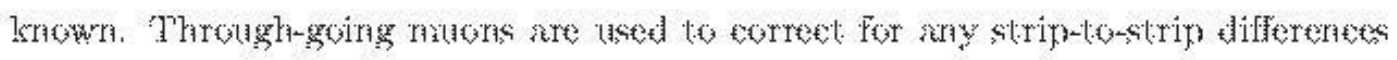

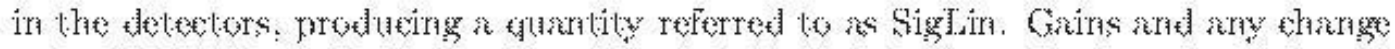

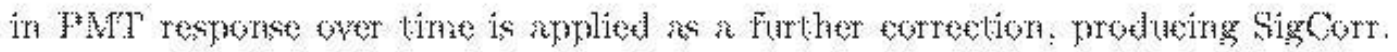

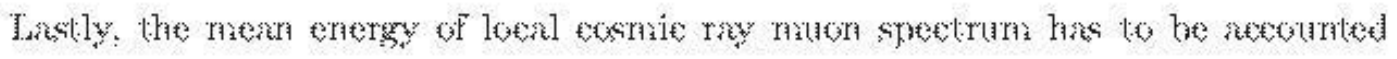

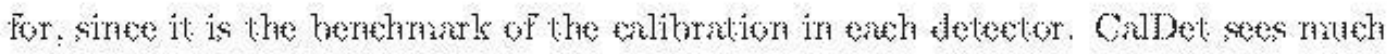

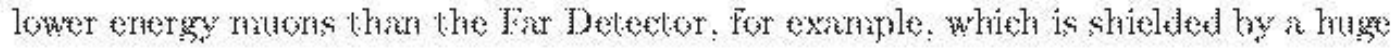

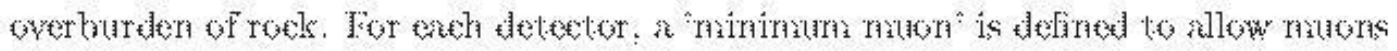


in all detectors to be compared on a relative basis. This quantity is defined as a MIP, and is equivalent to SigCorr multiplied by some constant for each detector. Finally to achieve a full, inter-detector absolute energy calibration, the CalDet is used to compare beam muons of known energy with these MIPs. (See Section 5.1 for more details on the energy calibration.)

The means and values of the root-mean-square are extracted from the spectra to provide the average energy deposited in that strip across the entire muon sample, and it's associated error, given by:

$$
\frac{\text { RMS }}{\sqrt{\text { Numberof Entries }}}
$$

These points can be plotted for the entire muon track producing a characteristic shape where the energy deposition rises markedly as the muon comes to a stop. These plots are typically portrayed using the number of planes from the end of the track; a given muon may have a range that is an even or odd number of planes in length so they must be arranged such that they all stop in the same place. In this case, the point that the muons stop is on the left hand side of the plot and the point of entry into the detector is on the right. The next stage is to apply a Bethe-Bloch fit to the results.

\begin{tabular}{|c|c|c|c|c|}
\hline $\begin{array}{c}\mathrm{p} \\
\mathrm{GeV} / \mathrm{c}\end{array}$ & $\begin{array}{c}\text { Ionisation } \\
\mathrm{MeVcm}^{2} / \mathrm{g}\end{array}$ & $\begin{array}{c}\text { Bremstrahlung } \\
\mathrm{MeVcm}^{2} / \mathrm{g}\end{array}$ & $\begin{array}{c}\text { Pair Production } \\
\mathrm{MeVcm}^{2} / \mathrm{g}\end{array}$ & $\begin{array}{c}\text { Photonuclear } \\
\mathrm{MeVcm}^{2} / \mathrm{g}\end{array}$ \\
\hline 1 & 1.581 & 0.001 & & 0.000 \\
4 & 1.806 & 0.004 & 0.003 & 0.002 \\
10 & 1.942 & 0.014 & 0.014 & 0.004 \\
20 & 2.032 & 0.033 & 0.038 & 0.008 \\
\hline
\end{tabular}

Table 5.6: Showing the various contributions to the total energy loss that a muon experiences travelling through iron, at various energies. The data is taken from Groom [77]. 

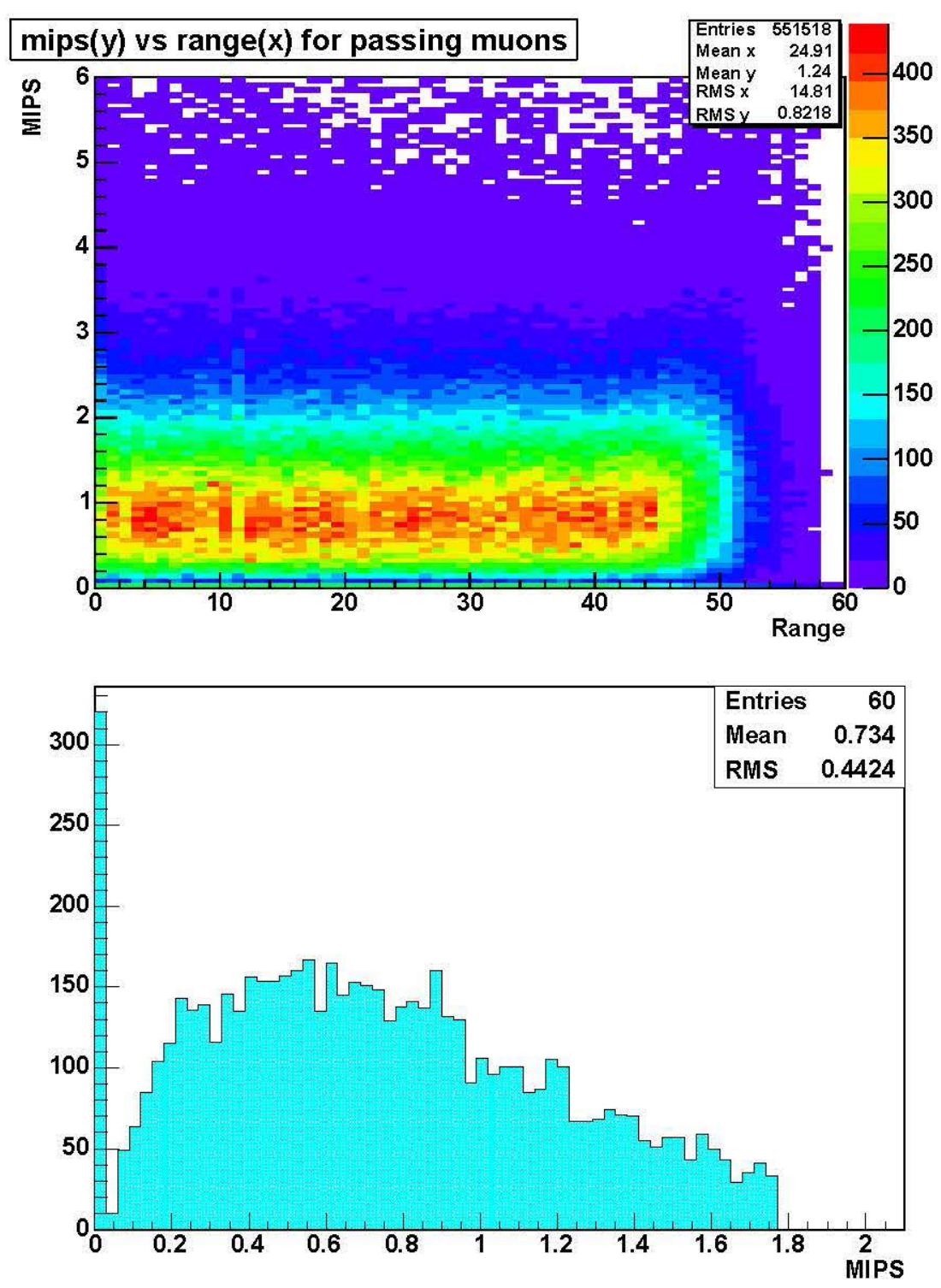

Figure 5.45: Top: Showing the distribution of hits and the energy deposited by $1.8 \mathrm{GeV}$ muons travelling through the detector. This is the complete, untruncated distribution. Bottom: Showing a one-plane slice (plane 31) of the plot above. The hit distributions from each plane are truncated at $90 \%$ and the mean and RMS are extracted. 


\begin{tabular}{|c|c|c|c|c|}
\hline $\begin{array}{c}\mathrm{p} \\
\mathrm{GeV} / \mathrm{c}\end{array}$ & $\begin{array}{c}\text { Ionisation } \\
\mathrm{MeVcm}^{2} / \mathrm{g}\end{array}$ & $\begin{array}{c}\text { Bremstrahlung } \\
\mathrm{MeVcm}^{2} / \mathrm{g}\end{array}$ & $\begin{array}{c}\text { Pair Production } \\
\mathrm{MeVcm}^{2} / \mathrm{g}\end{array}$ & $\begin{array}{c}\text { Photonuclear } \\
\mathrm{MeVcm}^{2} / \mathrm{g}\end{array}$ \\
\hline 1 & 2.048 & 0.000 & & 0.000 \\
4 & 2.275 & 0.001 & 0.001 & 0.002 \\
10 & 2.414 & 0.004 & 0.005 & 0.005 \\
20 & 2.509 & 0.009 & 0.010 & 0.009 \\
\hline
\end{tabular}

Table 5.7: Showing the various contributions to the total energy loss that a muon experiences travelling through polystyrene, at various energies. The data is taken from Groom [77].

\subsubsection{Bethe-Bloch Fitting}

The Bethe-Bloch formula is quite complex and an appropriate fitting function is also necessarily complicated. An initial set of parameters are entered, in particular the densities and thicknesses of the various detector components that the particles travel through. The method then advances through the detector in small steps, calculating the energy loss over each small distance. This is necessary since the energy lost at each step is dependent on the total remaining energy (i.e. a step determines the starting point of the calculation for the next step). There is 1 step for the aluminium, then 10 steps of scintillator followed by another step of aluminium and finally 100 steps of steel. The particle's energy and momentum are tracked at each step and the summation of these energy loss steps is the total $\mathrm{dE} / \mathrm{dX}$.

The initial parameters can be fixed or allowed to float, allowing for a best fit to the observed data. A fit to the nominal parameters at $1.8 \mathrm{GeV}$ is shown in Figure 5.46. An improvement of the fit can be seen by allowing the parameters to float a little way from their nominal values. In some cases this allows a higher degree of accuracy on the measurement to be obtained, depending on the resolution and by what means they were previously measured. The steel thickness for example was measured in a few places on a spare plane. There was some variation between measurements, suggesting that the nominal value may not be the most accurate. The best fit to the data was achieved using $1.8 \mathrm{GeV}$ muons, since they stopped well away from the detector edge. The best fit parameters were extracted from this data 
point and propagated to the subsequent, higher energy fits. Figure 5.47 shows this $1.8 \mathrm{GeV}$ fit and a comparison is made in Table 5.9. Some examples of Bethe-Bloch fits to higher energies are shown in Figure 5.48.

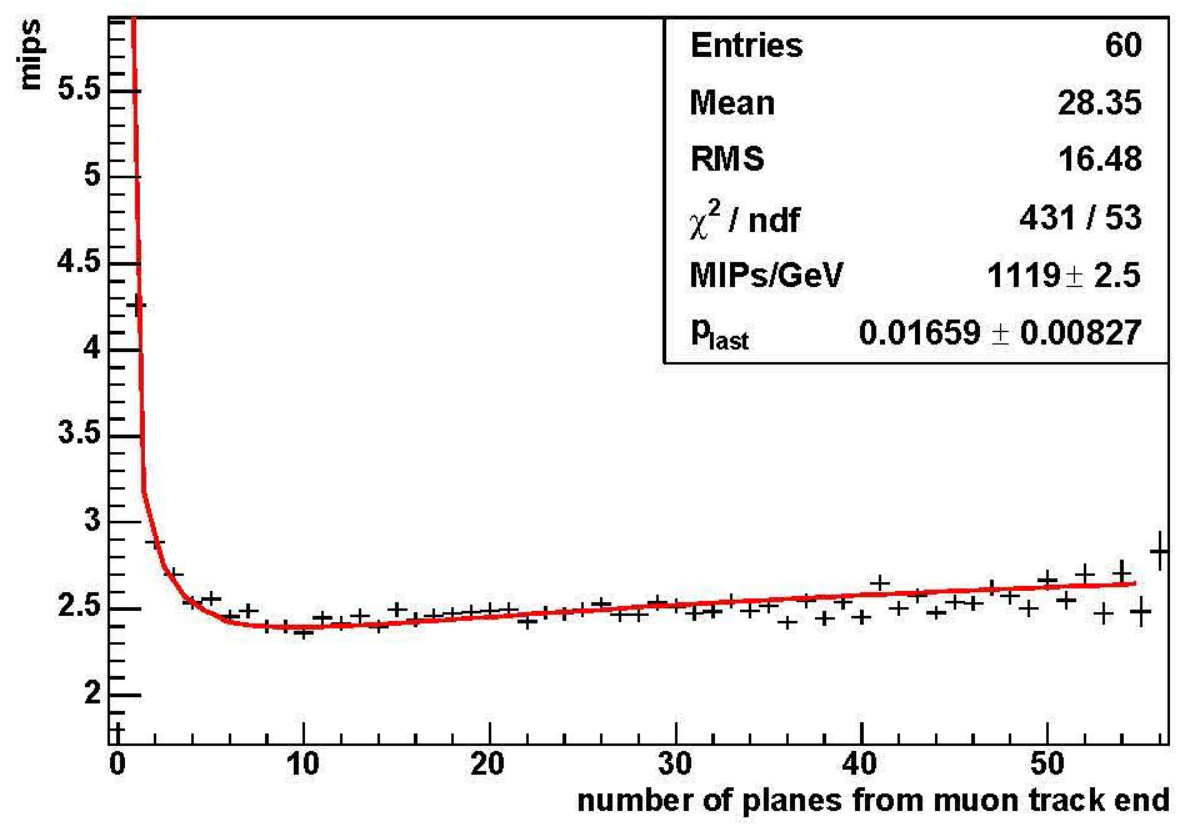

Figure 5.46: Showing the initial $\mathrm{dE} / \mathrm{dX}$ curve, with the parameters fixed at their nominal settings. In this case, the nominal settings are the design specifications of the CalDet components.

\begin{tabular}{|c|c|c|c|}
\hline Plane Component & Thickness $(\mathrm{cm})$ & Energy Loss(MeV) & Percentage Loss \\
\hline Steel & 2.50 & 28.9 & $92.3 \%$ \\
Polystyrene & 1.00 & 1.90 & $6.2 \%$ \\
Aluminium & 0.02 & 0.44 & $1.5 \%$ \\
Air & 2.30 & 0.01 & $0.0 \%$ \\
\hline
\end{tabular}

Table 5.8: Showing the energy losses of a minimum ionizing particle as it travel through a plane of CalDet. The values shown are nominal: the Bethe-Bloch fitting procedure allows the values to fluctuate a little to account for small variations from the design specifications. These variations do not change on a plane-by-plane basis. 


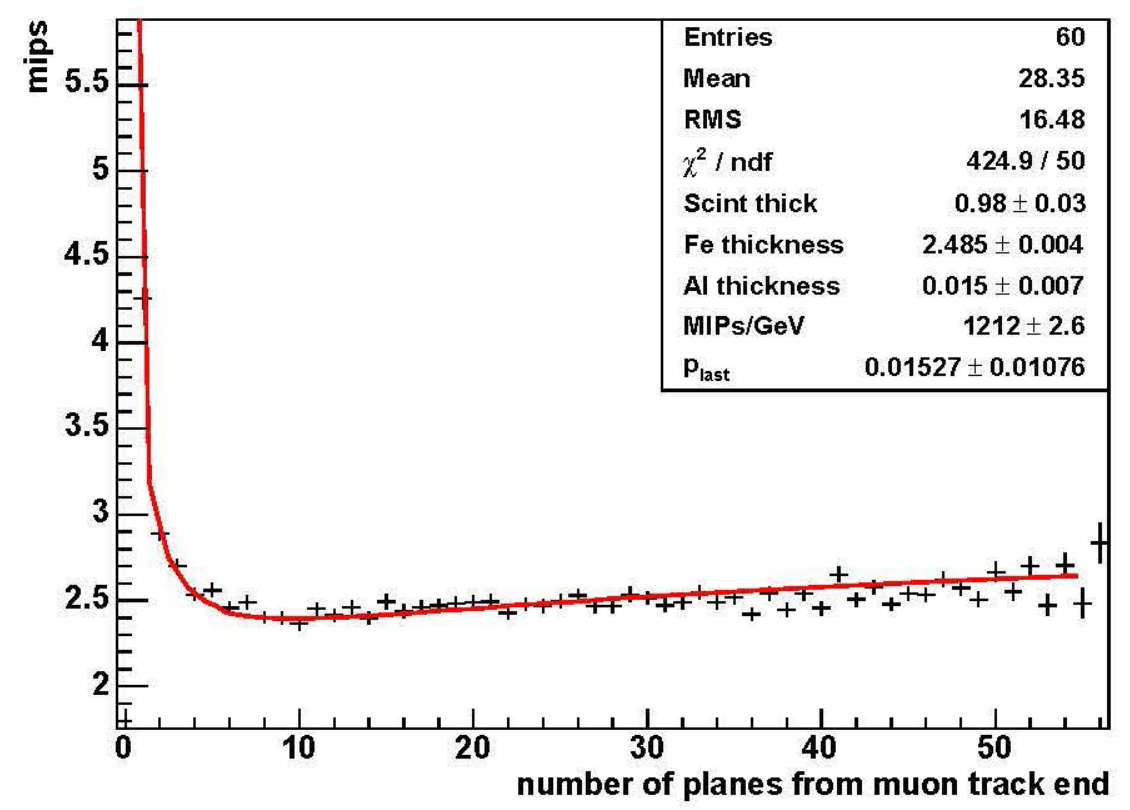

Figure 5.47: Showing the modified values of the fitting parameters, after the minimisation procedure of the fitting itself had been completed. These values have a higher accuracy and are considered to be a better representation of the actual component parameters at CalDet.

\begin{tabular}{|c|c|c|}
\hline Parameter & Nominal Value & Fit Value \\
\hline Steel thickness & $2.50 \mathrm{~cm}$ & $2.484 \pm 0.004 \mathrm{~cm}$ \\
Polystyrene thickness & $1.00 \mathrm{~cm}$ & $0.98 \pm 0.03 \mathrm{~cm}$ \\
Aluminium thickness & $0.02 \mathrm{~cm}$ & $0.015 \pm 0.007 \mathrm{~cm}$ \\
Light Level & $1200 \mathrm{MIPS} / \mathrm{GeV}$ & $1212 \pm 2.6$ \\
\hline
\end{tabular}

Table 5.9: Showing the nominal fitting parameters for the Bloch-Fit and those extracted from the minimisation of the fitting procedure itself. 

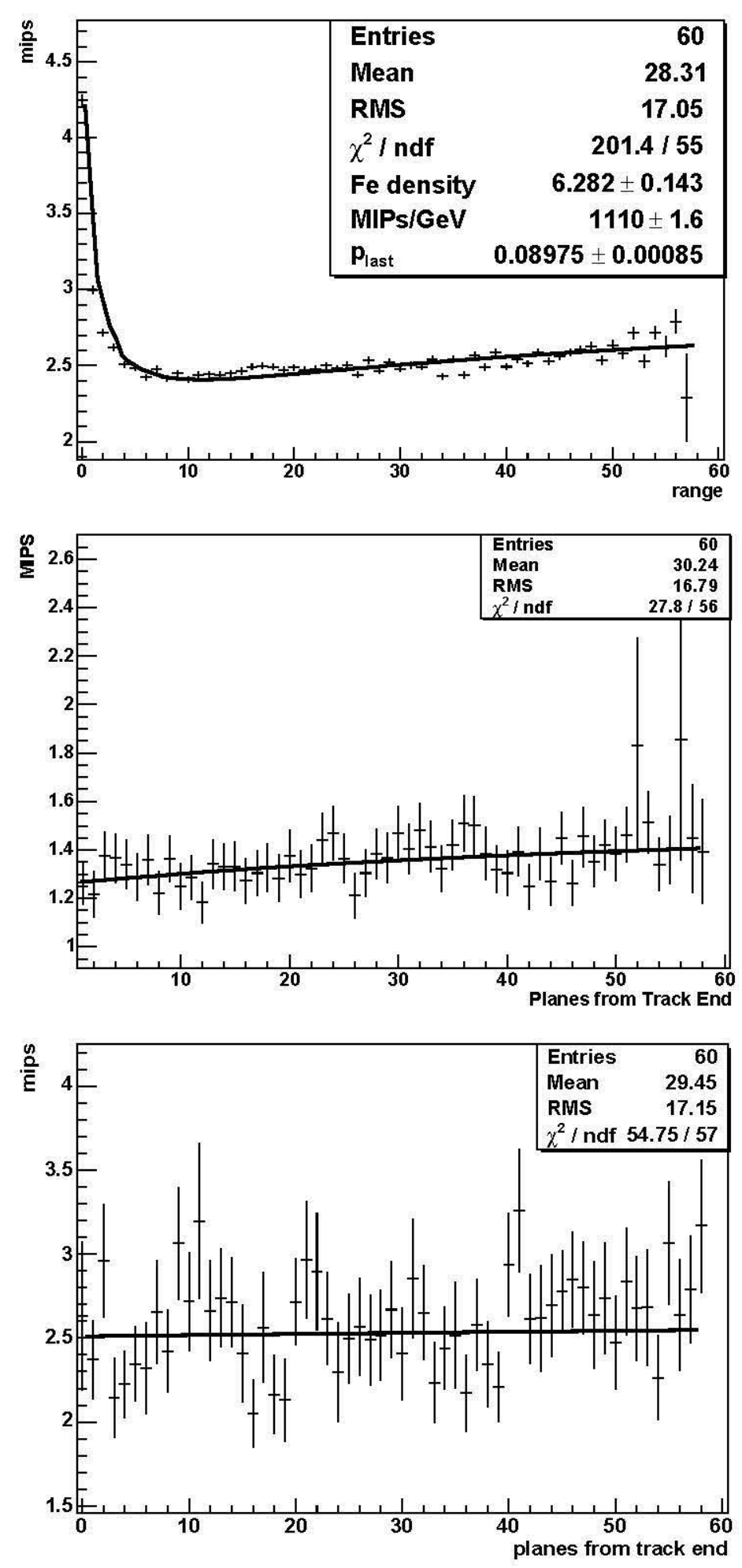

Figure 5.48: Showing the Bethe-Bloch fits to data at 1.8, 3 and $10 \mathrm{GeV}$. Above 2.2 $\mathrm{GeV}$, muons do not stop in the detector and the characteristic increase in energy deposition for stopping muons is not seen. The fit is therefore expected to be less acurate and requires optimised fit parameters that have been extracted from a better fit (i.e. $1.8 \mathrm{GeV}$ ). 
The results of the Bethe-Bloch fitting can be cross-checked against published results. Having made the modified fit to various energies, it can be tracked back to the first plane, or the point of entry of the muon into the detector. The data from this plane alone is unsatisfactory because 'splashing' was sometimes observed, that is a large number of low energy particles were observed to hit the front plane of the detector, but travelled no further. At the point of entry of the muon, it is closest to the nominal beam energy, before it begins to slow down in the detector. The energy deposition at the point of entry is then extracted from the fit and compared to the published Groom tables. This is shown in Figure 5.49.

The fit values of MIPS/GeV are also required to convert the energy seen in the detector (in MIPS) to visible energy (GeV). MIPS/GeV is a quantity often refered to as the 'light level'. Translating from the muon standard candle to visible energy, it can vary when the detector is moved or re-wired, for example. Temperature is also thought to have an effect $(\sim-0.3 \%)$ since it can alter the properties of the scintillator, although there is a time lag associated with this. These effects could be responsible for the consistent observation of more energy deposition in CalDet than expected from the Groom tables. Contamination from PS muons could also be partly to blame.

\subsection{Summary}

The range of muons in the MINOS detectors is the linchpin of the relative and absolute energy scale calibration. A good understanding of how they behave in the detectors is therefore crucial. In addition, some pions produced in neutral current interactions can simulate muons by travelling a significant distances in the detector before interacting.

At the near and far detectors, the only way to identify muons is via topological and energy cut parameters. At CalDet, there was also the possibility of using cerenkov detectors, although their efficiency was found to be dependent on the energy of the muons travelling through them. In general, the cerenkov detectors were found to be around $85 \%$ efficient at identifying on momentum muons. It was also possible to determine the fraction of muons being produced in various areas of the 


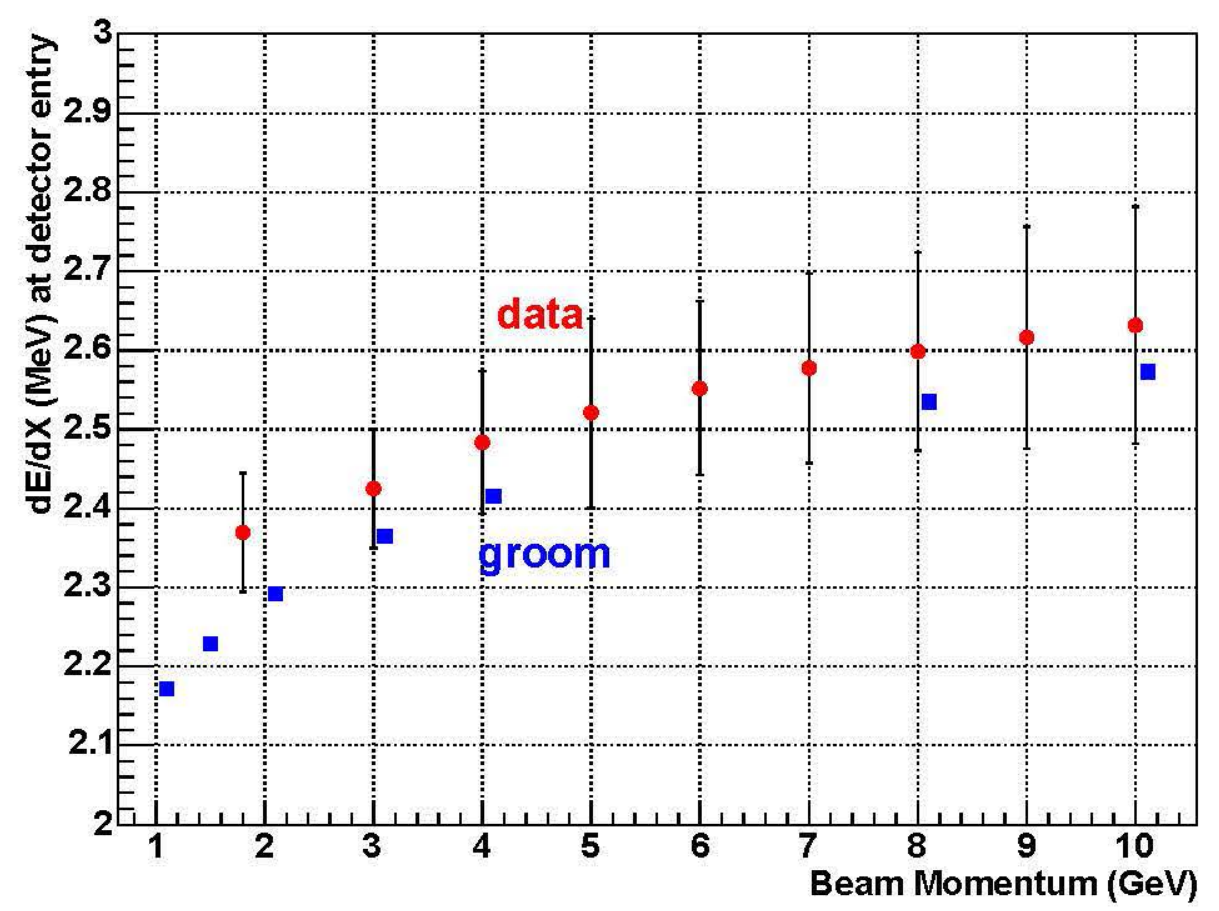

Figure 5.49: Showing the $\mathrm{dE} / \mathrm{dX}$ for data muons at the point of their entry into the detector, against the data presented in the Groom et al tables [77].

beamline by using the cerenkov counters.

Muons arise from the decays of pions in the CERN test beams and as such are not mono-energetic. Shape and energy cuts must be applied to the data to separate pions and muons and Monte Carlo must be used to determine the appropriate cut parameters. With the addition of timing and fiducial cuts the various particle components of the test beams could be separated.

Since the beginning of the running of CalDet at CERN, it has been known that the conventional Monte Carlo simulations do not simulate the observed muon spectrum well. It has been shown that this is because the upstream region of the beamline is not simulated well, with there being many more on-momentum muons than previously thought. Adjustment of the collimators has showed that this is more than likely due to 'scraping': additional muons being produced by pion interactions with the collimators, that were absent from the simulation. The discrepancy in the number of muons produced in the upstream region was as much as $100 \%$ at times, depending on the collimator settings. 
Muons travelling though the MINOS detectors deposit energy in a manner described by the Bethe-Bloch formula. It has been shown that a Bethe-Bloch fitting function describes the data well and it in good agreement with the tables published by Groom et al. 


\section{Chapter 6}

\section{The NEMO-3 Experiment}

\subsection{Introduction}

NEMO-3 is a neutrinoless double beta decay $(0 \nu \beta \beta)$ experiment. If this interaction is observed, it means that the neutrino is a Majorana particle, it is its own antiparticle and lepton number is not a strictly conserved quantity. This would be a very important result for neutrino physics.

The mechanism of $0 \nu \beta \beta$ could occur in a number of ways. A majorana mass term for the neutrino would allow it to occur through the V-A interaction, with a helicity flip of the neutrino. It could also occur through a $\mathrm{V}+\mathrm{A}$ interaction to a $2^{+}$excited state which requires as yet unseen right handed currents. Another class of mechanisms that could contribute are those that include the emission of one or more Majorons, a boson that would be responsible for the spontaneous symmetry breaking of lepton number.

\subsection{Detector Description}

NEMO-3 is a calorimeter that also has particle tracking capabilities. It is cylindrical, around $3 \mathrm{~m}$ high with a diameter of $5 \mathrm{~m}$ and is composed of 20 wedge shaped 'sectors'. Each sector houses a $\beta \beta$ emitting isotope in the form of a 'foil'. NEMO-3 contains various quantities of 7 specially chosen isotopes; these are shown in Figure 6.1 . 


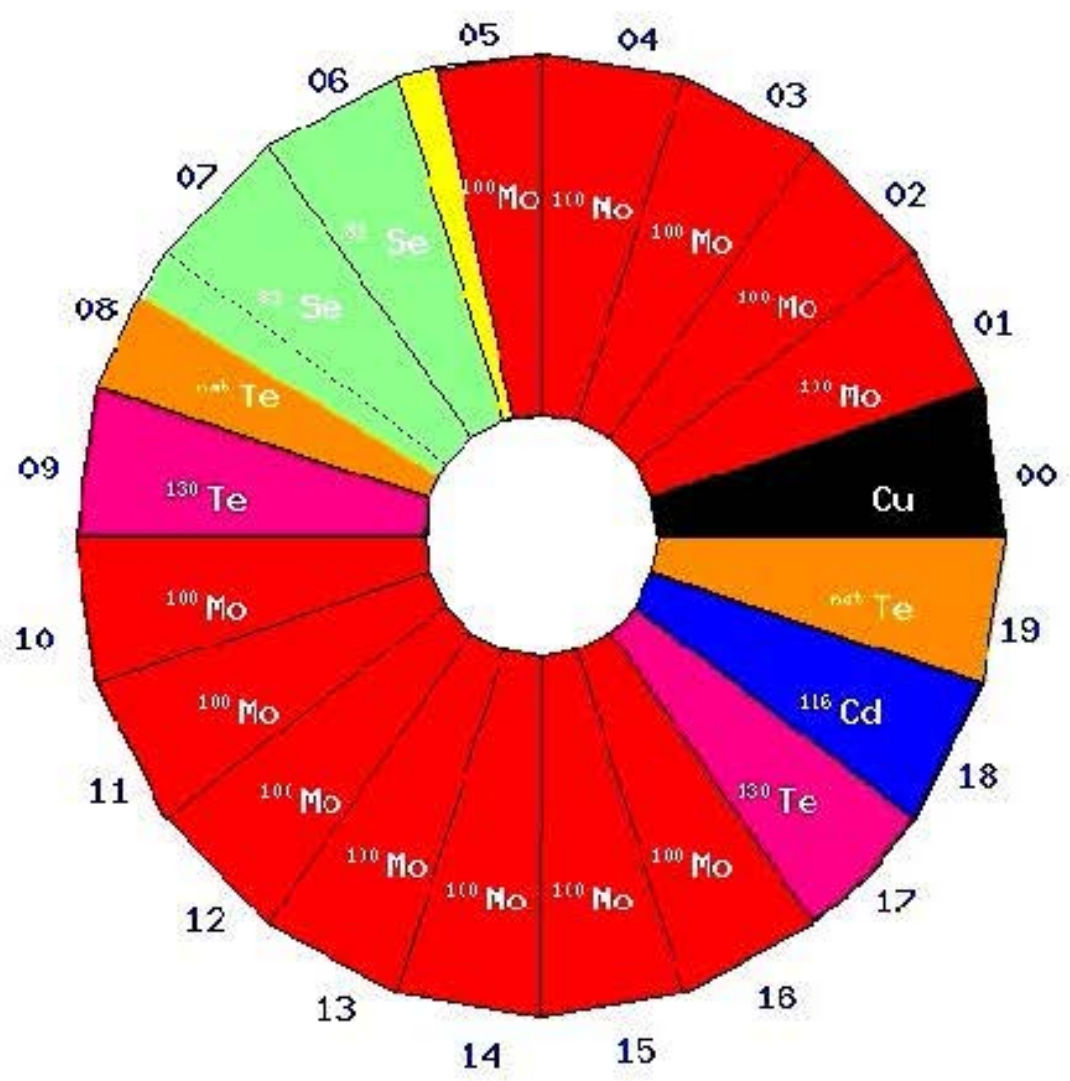

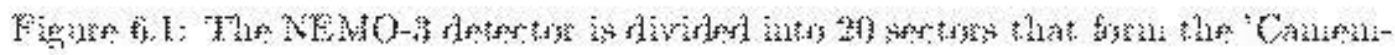

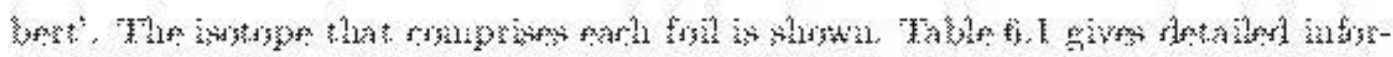

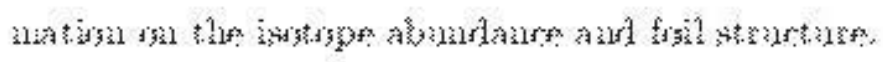

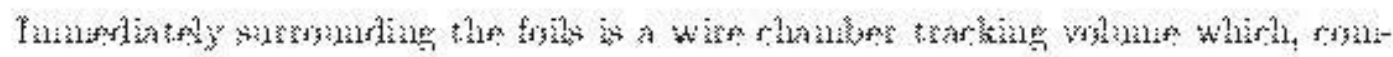
Wing w w

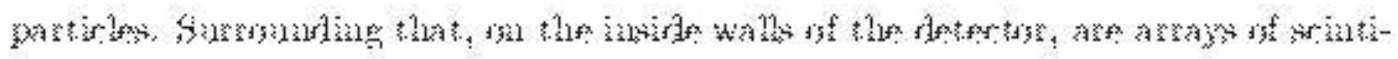

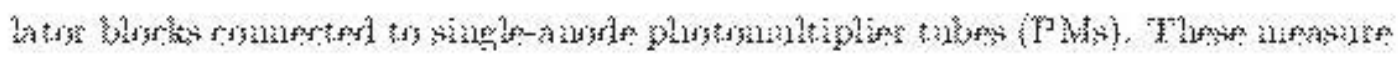

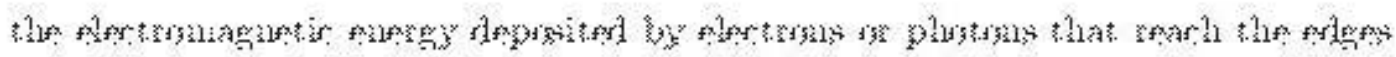

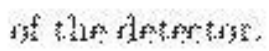

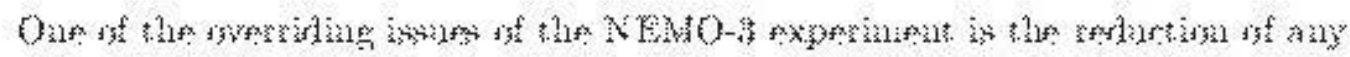

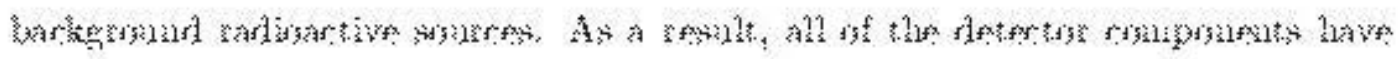

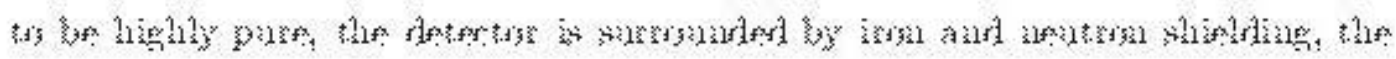

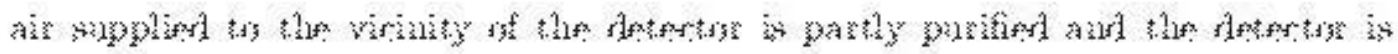


located $1780 \mathrm{~m}$ (4850 m.w.e.) underground in the Modane Underground Laboratory (LSM). Situated in the Fréjus tumel between France and Italy, the detector has been routinely taking data since June 2002 in this low cosmic background environment.

\subsubsection{Source Foils}

\begin{tabular}{|c|c|c|c|c|c|c|c|}
\hline \multirow[b]{2}{*}{ Isotope } & \multirow[b]{2}{*}{ Type } & \multirow{2}{*}{$\begin{array}{c}\text { Mass } \\
(\mathrm{g})\end{array}$} & \multirow{2}{*}{$\begin{array}{l}\text { Number } \\
\text { of Sectors }\end{array}$} & \multirow{2}{*}{$\begin{array}{c}\mathrm{Q} \\
(\mathrm{keV})\end{array}$} & \multicolumn{2}{|c|}{ Activity $(\mathrm{mBq} / \mathrm{kg})$} & \multirow{2}{*}{$\begin{array}{c}\text { Processes } \\
\text { Studied }\end{array}$} \\
\hline & & & & & $\mathrm{Tl}$ & $\mathrm{Bi}$ & \\
\hline${ }^{100} \mathrm{Mo}$ & Foil & 2479 & 5 & 3034 & $<0.104$ & $<0.300$ & $0 \nu \beta \beta, 2 \nu \beta \beta$ \\
\hline${ }^{100} \mathrm{Mo}$ & Comp. & 4435 & 7 & 3034 & $<0.140$ & $<0.090$ & $0 \nu \beta \beta, 2 \nu \beta \beta$ \\
\hline${ }^{82} \mathrm{Se}$ & Comp. & 932 & 2.3 & 2995 & $0.4 \pm 0.1$ & $1.2 \pm 0.5$ & $0 \nu \beta \beta, 2 \nu \beta \beta$ \\
\hline${ }^{116} \mathrm{Cd}$ & Foil & 405 & 1 & 2805 & $<0.5$ & $<1.5$ & $0 \nu \beta \beta, 2 \nu \beta \beta$ \\
\hline${ }^{130} \mathrm{TeO}_{2}$ & Comp. & 454 & 1.8 & 2829 & $<0.51$ & $<0.68$ & $0 \nu \beta \beta, 2 \nu \beta \beta$ \\
\hline${ }^{150} \mathrm{Nd}_{2} \mathrm{O}_{3}$ & Comp. & 36.6 & 0.14 & 3367 & $10 \pm 2$ & $<3.3$ & $0 \nu \beta \beta, 2 \nu \beta \beta$ \\
\hline${ }^{96} \mathrm{ZrO}_{2}$ & Comp. & 9.4 & 0.03 & 3350 & $<10$ & $<17$ & $2 \nu \beta \beta$ \\
\hline${ }^{48} \mathrm{CaF}_{2}$ & S.P. & 7.0 & 0.03 & 4272 & $<2$ & $<4$ & $2 \nu \beta \beta$ \\
\hline${ }_{n a t} \mathrm{TeO}_{2}$ & Comp. & 207 & 1.7 & 2829 & $<0.333$ & $<0.167$ & background \\
\hline${ }^{n a t} \mathrm{Cu}$ & Foil & 621 & 1 & - & $<0.033$ & $<0.117$ & background \\
\hline
\end{tabular}

Table 6.1: Various information about the source foils used in the NEMO-3 detector. 'Comp.' refers to composite foils; typically metal powder glued to mylar sheets. 'S.P.' refers to disks of powder that are sealed between two mylar sheets.

The NEMO experiment originally set out to investigate $0 \nu \beta \beta$ in Molybdenum. Since then purification techniques have improved significantly, and now several isotopes that exhibit $2 \nu \beta \beta$ are being investigated. In addition, the purpose of the ${ }^{n a t} \mathrm{TeO}_{2}$ and ${ }^{n a t} \mathrm{Cu}$ is to measure the external background. Table 6.1 lays out some information about the isotopes used. The activites of these materials are very low, and have to be, to mimimise any contamination that could pollute the $0 \nu \beta \beta$ signal. This is also why isotopes with high $Q$ values are favoured. The purity of the samples can be attributed to the incredibly stringent physical and chemical purification processes that the sources go through. 


\subsubsection{Tracking Wire Chamber}

The NEMO-3 detector has 6180 open octagon drift cells operating in geiger mode which provide three dimensional tracking of charged particles. Each cells is composed of 1 anode wire (at $\sim 1800 \mathrm{~V}$ ) and 9 or 10 cathode wires (at ground, 0V), one or two of which are shared with the adjoining cells. The wires are $270 \mathrm{~cm}$ long, made from $50 \mu m$ diameter stainless steel and run from the top wall to the bottom of the detector. Each half sector has 9 rows of cells, split into groups of 4,2 and 3 moving away from the central foil. In between groups are scintillator blocks. See Figure 6.2 The wire chamber has been designed to maximise its transparency to the charged particles travelling through it.

Charged particles ionise the gas in the wire chamber as they traverse it. The electrons produced drift towards the anode wire at about $1 \mathrm{~cm} / \mu \mathrm{s}$ and by the time they are around $100 \mu \mathrm{m}$ away from it, they have enough kinetic energy to ionise the gas themselves. An avalanche of electrons known as a Geiger plasma is produced in the vicinity of the anode wire and, when it arrives there, propagates along it in both directions at about $6 \mathrm{~cm} / \mu \mathrm{s}$. The time difference between the plasma arriving at the top and bottom of the wire gives the longitudinal position of the particles [91].

To reduce multiple scattering of particles, the detector is filled with a specialized mixture of gases: 95\% helium, 4\% ethanol and 1\% argon. Ethanol and argon act as quenchers, to achieve a perfect balance between width and amplitude of signal. The pressure inside the detector is 7 mbar above local atmospheric.

\subsubsection{Calorimeter}

The NEMO-3 calorimeter walls have three main functions:

- To measure the energy of electrons/positrons in the energy range $150 \mathrm{keV}$ $12 \mathrm{MeV}$

- To measure the energy of photons in the energy range $80 \mathrm{keV}-12 \mathrm{MeV}$

- To measure time-of-flight and act as a trigger

There are 1940 blocks of scintillator in total with 34 on the internal wall, 39 on the external wall and 12 on the top and bottom walls, per sector. Blocks are $20 \times 20 \times 10 \mathrm{~cm}$ 


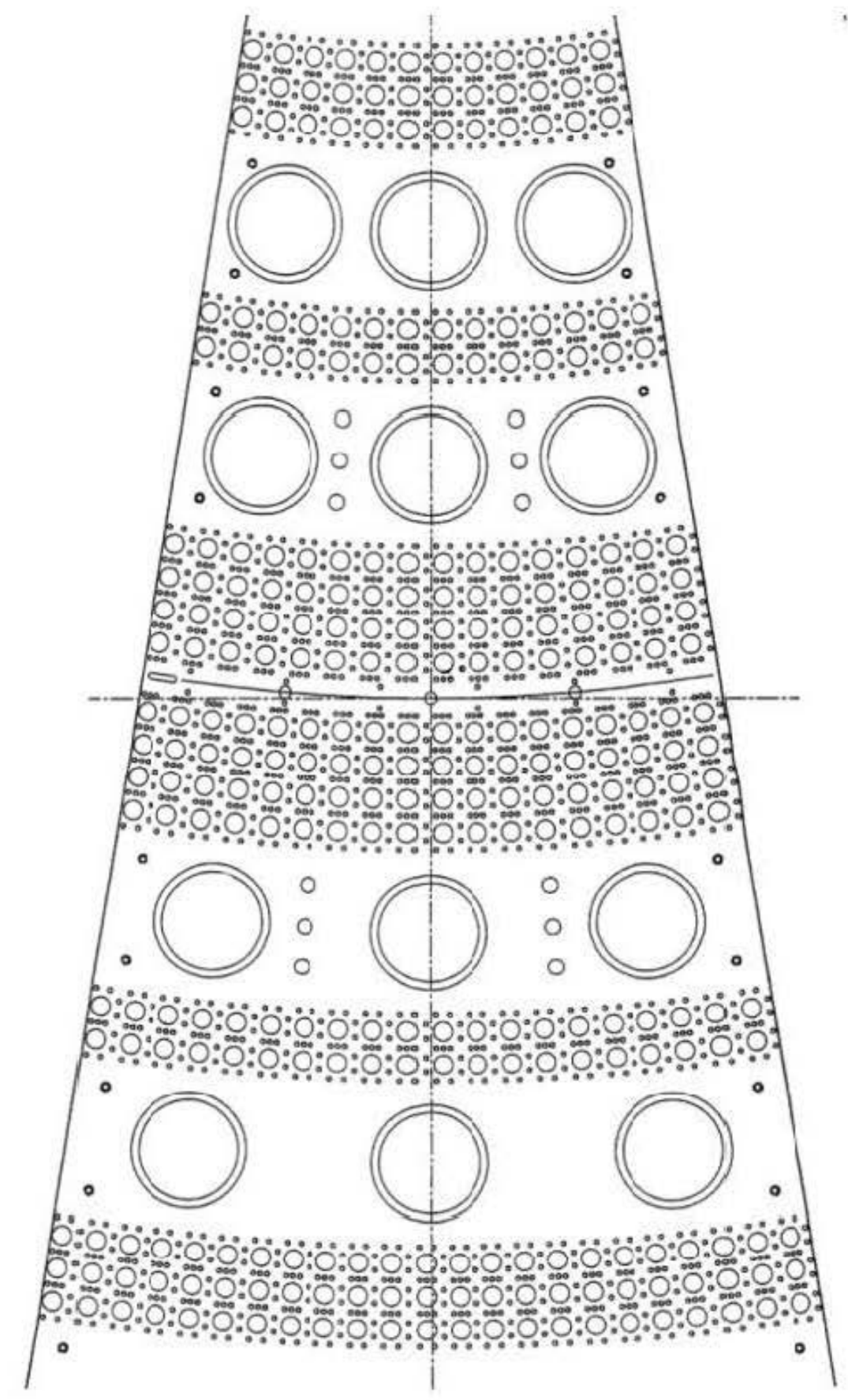

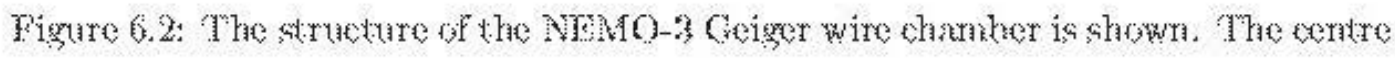

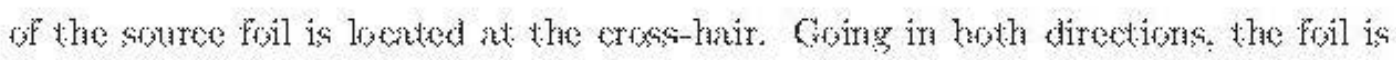

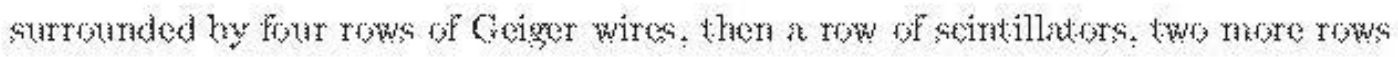

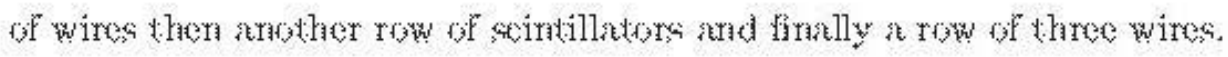


on external walls and $15 \times 15 \times 10 \mathrm{~cm}$ on internal walls. They are mostly made from $98.49 \%$ polystyrene, doped with $1.5 \%$ scintillating agent p-Terphenyl and $0.01 \%$ wavelength shifter, POPOP. They are wrapped in mylar for protection and light tightness and a teflon band at the interface of scintillator blocks reflects lost light back towards the respective PM they are attached to. NEMO-3 uses 3-inch and 5-inch Hamamatsu PMs with special low activity glass. They are housed inside black plastic boxes to minimise contamination from ambient light and are protected from the magnetic field by cylindrical shaped shields made from high nickel steel ( $\mu$-metal). Figure 6.3 shows the layout of the NEMO-3 calorimter.

\subsubsection{Electronics}

The drift cells and the calorimeter use separate electronics meaning that the DAQ and triggering can be dependent on either or both. 160 distribution boards supply HV from CAEN power supplies to the drift cells and receive signals back from the anode and cathode wires. These signals are passed to $160 \mathrm{VME}$ acquistion boards which digitize them. A signal on the anode wire starts the counting of an anode TDC and two cathode TDCs, one corresponding to the top of a given wire and one from the bottom. The TDCs are stopped when a signal is received from its respective location, up to a maximum of $6.14 \mu \mathrm{s}$ later. The anode TDC is stopped by the acquisition trigger. Up to $710 \mu$ s after this, other anodes can still fire, setting off a slow TDC which is designed to record alpha particles.

The CAEN supplies also power the PMs. Three PMs are supplied by one HV channel through distribution boards, so resistors on these boards ensure that each PM receives the correct voltage. The PM signal goes directly to acquisition boards (1 per half sector) which begin charge integration and TDCs once a low threshold has been reached. The digitization does not begin until a high threshold is passed at which point the trigger is signalled that a PM has fired.

The trigger has three levels. The first is based on PM hit multiplicity, the second is based on track recognition in the tracking wire chamber and the third trigger is based on associating hit scintillators with tracks. 


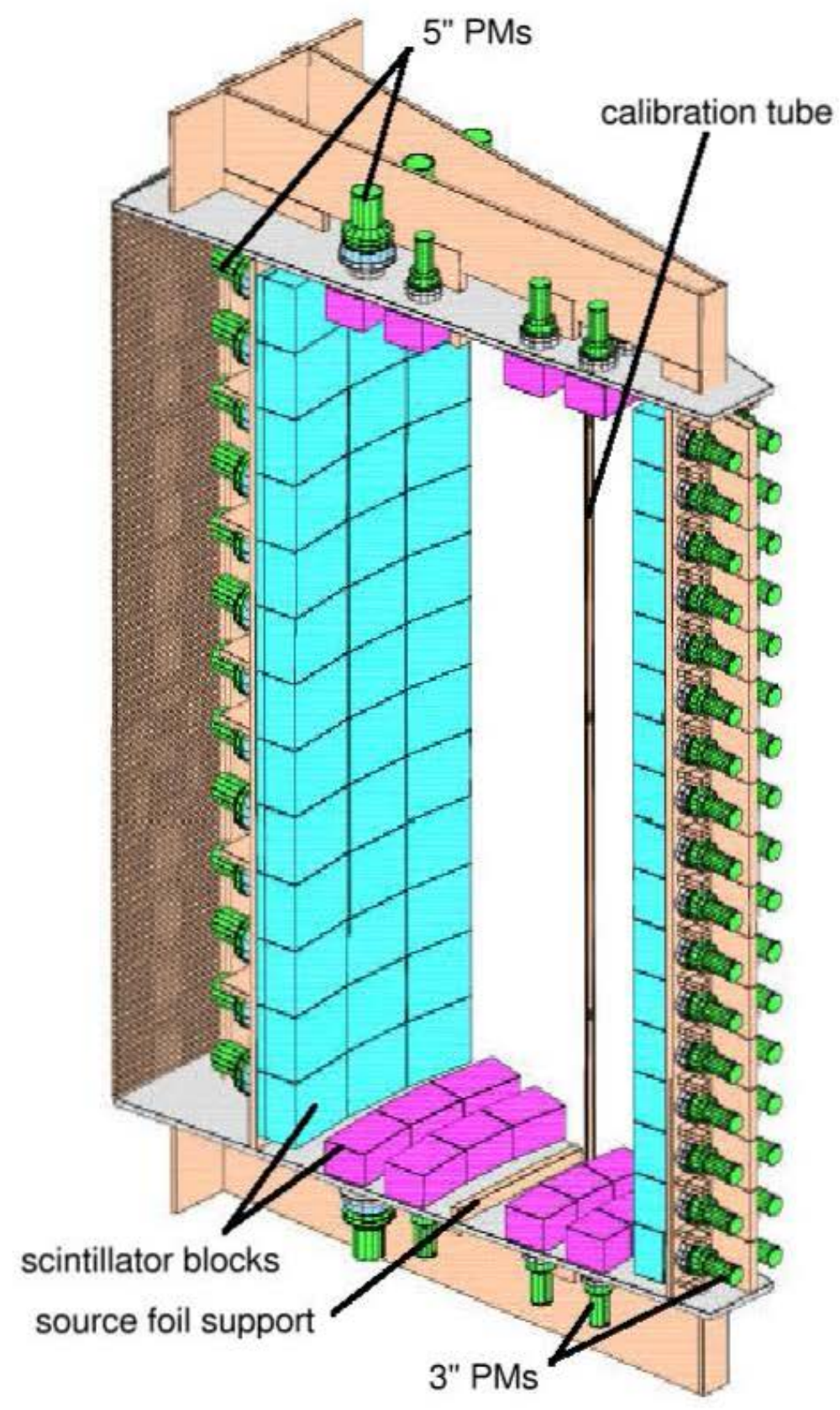

Figure 6.3: Wach sector is exmposed in the same way with the souree foil in the exntre and scintillator blocks and PMs an the inner, outer, top and bottom walls. The geiger wires are not shown in this diagram for elarity. 


\subsubsection{Magnetic Field and Shielding}

It has been shown that high energy photons impinging on the source foils (from outside the detector or from neutron capture) can produce electron-positron pairs. This is dangerous since it can mimic the $0 \nu \beta \beta$ signal. However, a 20-30 Gauss vertical magnetic field is used in NEMO-3 which causes electrons and positrons to curve in different directions. Particle tracking can then be used to reject positrons at the $95 \%$ level. The field is created by interconnected copper rods outside the external wall which form a solenoid.

Outside the solenoid is a $20 \mathrm{~cm}$ thick low activity iron shield to reduce neutron and $\gamma$-ray flux into the detector. Ouside of this is water and wood shielding to thermalise neutrons before they arrive at the steel.

\subsection{Calibration}

Absolute energy and time calibration of the detector is a time consuming process that requires specialised runs to be taken. As a result, it is only performed 2 or three times a year. On a much shorter time scale conditions such as PM gains can vary, so a simple daily calibration is conducted that can then be used to correct to the absolute reference calibrations. A special laser based system is used to calibrate the calorimeter. A small bulb of scintillator is used to convert a laser pulse into a signal that simulates a 1 electron event. The signal is sent via optical fibres to all the PMs in the detector and 6 reference PMs that are continuously exposed to ${ }^{207} \mathrm{Bi}$ sources, monitoring the $976 \mathrm{keV}$ conversion electrons. Energy calibration to an accuracy of 1\% can be achieved by comparing the laser peak position in the reference PMs to the signal from the Bi sources and a mean value of the peak position as seen by the PMs of the detector.

For the longer absolute calibration, the calibration tubes are used. These are copper tubes, one present in each sector, that can be used to deliver sources inside the detector. Three energy points can be measured to ensure the linearity of the detector in the most important region; 482 and $976 \mathrm{keV}$ conversion electrons from

${ }^{207} \mathrm{Bi}$ and $2283 \mathrm{keV}$ from the end point spectrum of ${ }^{90} \mathrm{Y}$. Timing calibration is 
performed using an intense ${ }^{60} \mathrm{Co}$ source which emits $1332 \mathrm{keV}$ and $1173 \mathrm{keV}$ photons in coincidence.

\subsection{NEMO Physics}

NEMO-3 is searching for $0 \nu \beta \beta$ in ${ }^{100} \mathrm{Mo},{ }^{82} \mathrm{Se},{ }^{116} \mathrm{Cd},{ }^{130} \mathrm{Te},{ }^{150} \mathrm{Nd},{ }^{96} \mathrm{Zr}$ and ${ }^{48} \mathrm{Ca}$. The experiment can search for the effective Majorana electron neutrino mass $<m_{v}^{\epsilon}>$ down to the level of $0.1 \mathrm{eV}$. If no signal is detected, a limit can be set based on the half-life, from the relation:

$$
\left[T_{1 / 2}^{a_{v}}\right]^{-1}=G^{0 v}\left|M^{0 w}\right|^{2} \frac{m_{v}^{e}>^{2}}{m_{e}^{2}}
$$

where $G^{\text {an }}$ is a calculable phase-space factor proportional to the transition energy $Q_{\beta \beta}^{5}$ and $M^{0 v}$ is the nuclear matrix element of the isotope in question. Nuclear matrix element calculations have large uncertainties meaning that a mass limit of 0.1 $\mathrm{eV}$ would correspond to a $0 \nu \beta \beta$ half-life of order $10^{25}$ years for ${ }^{100} \mathrm{Mo}$. Hence isotopes with higher $Q_{\beta 3}$ produce a higher $G^{0 v}$ as well as being further from background contamination signals.

After around 389 days data taking (analysed and results published), the NEMO-3 experiment has not observed evidence for $0 \nu \beta \beta$ has so far with its $\sim 7 \mathrm{~kg}$ of ${ }^{100} \mathrm{Mo}$ and $\sim 1 \mathrm{~kg}$ of ${ }^{82} \mathrm{Se}$. The corresponding limits are $\mathrm{T}_{1 / 2}(0 \nu \beta \beta) \geq 4.6 \times 10^{23}$ years for ${ }^{100} \mathrm{Mo}$ and $\mathrm{T}_{1 / 2}(0 \nu \beta \beta) \geq 1.0 \times 10^{23}$ years for ${ }^{82} \mathrm{Se}(90 \% \mathrm{C} . \mathrm{L}$.). With uncertainties in the nuclear matrix element calculations included, the limits on the effective Majorana neutrino mass are:

$$
\begin{aligned}
& { }^{100} \mathrm{Mo}<0.7-2.8 \mathrm{eV} \\
& { }^{82} \mathrm{Se}<1.7-4.9 \mathrm{eV}
\end{aligned}
$$

which is beginning to exclude the region suggested by the Heidelberg-Moscow ${ }^{76} \mathrm{Ge}$ experiment. [44]. Radon has been the most significant background to date; the inclusion of a radon-tight tent has decreased this by a factor of $\sim 10$. 


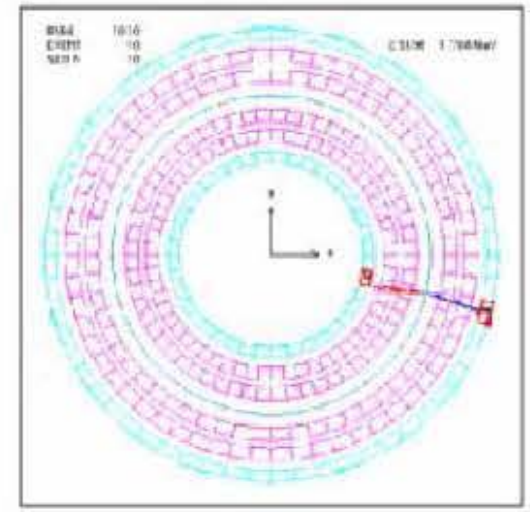

\section{Top view}
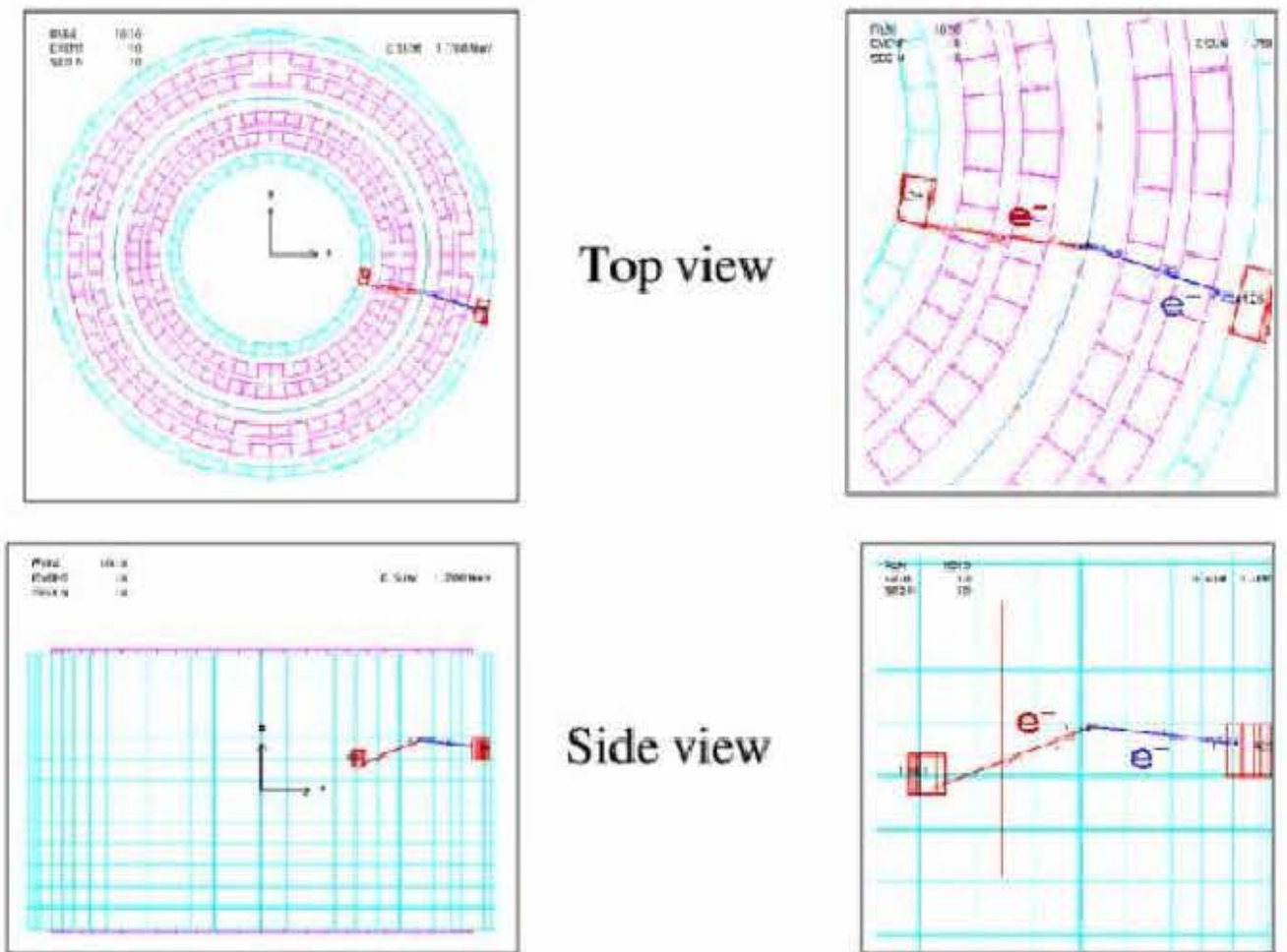

Side view

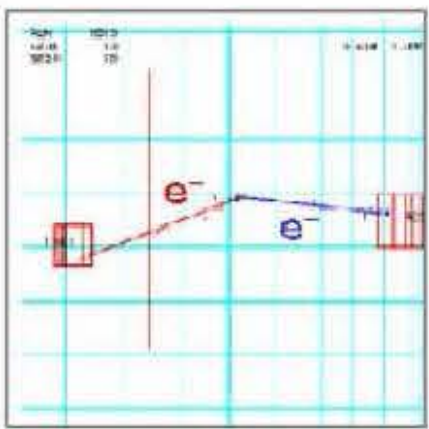

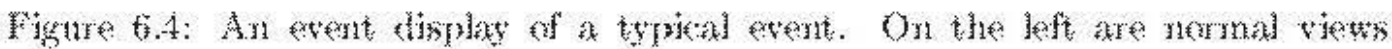

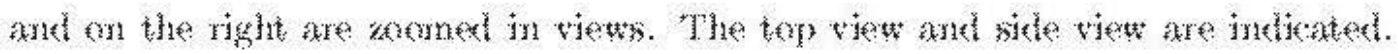

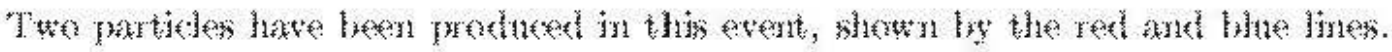

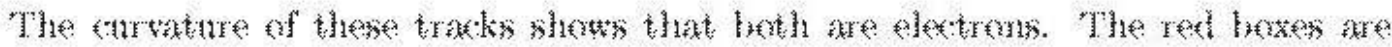

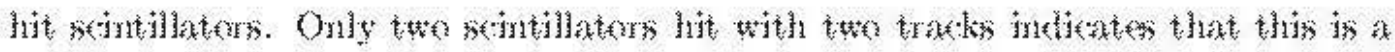
petexntal $2 v$,

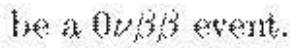

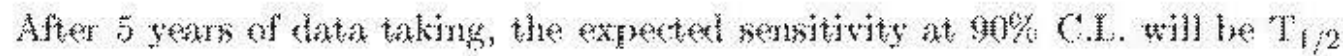

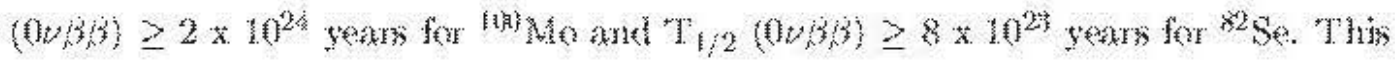

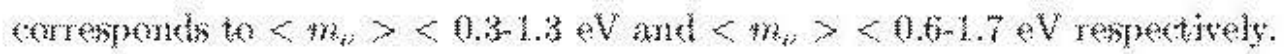




\section{Chapter 7}

\section{Background studies in the NEMO-3 experiment}

In NEMO-3, as with any other experiment, it is important to identify and study background signals. Wherever possible, the sources of these 'fake signals' should be reduced as much as possible, after which accurate estimates of their contamination of the real signals should be established. This chapter describes the study of two such

sources, ${ }^{214} \mathrm{Bi}$ and ${ }^{208} \mathrm{Tl}$ and their impact on the data when present in tiny amounts in the NEMO source foils. A measurement of the quantities of these isotopes is made using the detector itself, and an estimate is made of the number of signal-like events that result.

\subsection{Backgrounds of the NEMO-3 experiment}

The signal for $0 \nu \beta \beta$ in Molybdenum is two electrons, whose summed energy is $3.034 \mathrm{MeV}$. Any processes in or around the detector that produce a similar signal, or signals that could be interpreted as being the same, need to be reduced as much as possible. There are three main sources of such signals:

- The tail of the $2 \nu \beta \beta$ distribution

- Interactions of external neutrons and photons with the detector

- Natural radioactivity of materials used for the construction of the detector, 
within the LSM laboratory and the source foils themselves

\subsubsection{The tail of the $2 \nu \beta \beta$ distribution}

The summed energy spectrum of the two electrons emitted in $2 \nu \beta \beta$ is expected to extend as far as the $3.034 \mathrm{MeV} 0 \nu \beta \beta$ signal region. Some number of events in the extreme of the high energy tail of the $2 \nu \beta \beta$ distribution therefore represent a background that cannot be avoided. However, by studying the $2 \nu \beta \beta$ half-life of the isotopes involved, an estimate can be made of the number of events that would contaminate the $0 \nu \beta \beta$ signal. The relevant half-lives for ${ }^{100} \mathrm{Mo}$ and ${ }^{82}$ Se were measured by the NEMO-2 experiment:

$$
\begin{aligned}
& T_{1 / 2}^{2 \nu 3 \beta}\left({ }^{100} \mathrm{Mo}\right)=0.95 \pm 0.04(\text { stat. }) \pm 0.09(\text { syst. }) \times 10^{19} y \\
& T_{1 / 2}^{2 \nu \beta 3}\left({ }^{82} \mathrm{Se}\right)=0.83 \pm 0.10(\text { stat. }) \pm 0.07(\text { syst. }) \times 10^{20} y
\end{aligned}
$$

Within a $2.8-3.2 \mathrm{MeV}$ window, this equates to 1.1 events in $7 \mathrm{~kg}$ of ${ }^{100} \mathrm{Mo}$ per year and 0.1 events in $1 \mathrm{~kg}$ of ${ }^{82}$ Se per year [74].

\subsubsection{Neutrons and Photons}

Neutrons can be produced in matter (rock, detector components) by spontaneous fission of radioactive isotopes within the uranium and thorium decay chains, see Figure 7.1. These neutrons can be subsequently captured by nuclei in the detector frame and photons emitted of the order of $3 \mathrm{MeV}$ and above. NEMO-3 employs various layers of neutron shielding to minimise their effect.

The $\gamma$-ray flux in the LSM arises from natural radioactivity in the rocks, radiative neutron capture and bremstrahlung from cosmic ray muons. Photons that then interact with the source foils can produce $0 \nu \beta \beta$-like signals in a number of ways:

- The photon produces an electron-positron pair, the positron is subsequently mis-identified as an electron

- The photon produces an electron by the Compton effect which in turn produces another free electron by Möller scattering 
- Two electrons are produced by the Compton effect occuring twice

- An electron is produced inside the foil by the photoelectric effect which subsequently produces another free electron by Möller scattering

- An electron is produced by the Compton effect, and another by the scattered photon interacting via the photoelectric effect

\subsubsection{Radon}

Radon is a rare radioactive gas that is created by the decay of uranium and thorium (see Figure 7.1) that is present everywhere. It can seep out the rocks into the air and then can enter any regions of the detector that are not airtight, or become deposited on the detector via dust particles. A radon removal factory was employed to reduce levels of radon around the detector to less than $10-20 \mathrm{~Bq} / \mathrm{m}^{3}$. The main dangers of radon are through its daughter isotopes of ${ }^{214} \mathrm{Bi}$ and ${ }^{208} \mathrm{Tl}$ that are produced when it decays.

\subsection{4 ${ }^{214} \mathrm{Bi}$ and ${ }^{208} \mathrm{Tl}$}

There are traces of natural radioactivity in all parts of the detector. These arise from the daughter products of ${ }^{238}$ Uranium and ${ }^{232}$ Thorium which exist in tiny quantities everywhere since they have half-lives of millions of years. The most dangerous of these are ${ }^{214} \mathrm{Bi}$ and ${ }^{208} \mathrm{Tl}$ which produce $\beta$-rays in the $0 \nu \beta \beta$ energy window as they decay. There are three processes can then produce a second electron, such that their summed energy is $2.8-3.2 \mathrm{MeV}$ :

- Conversion electron: The nucleus of the $\beta$-emitter becomes excited as the decay occurs. The nucleus de-excites by ejecting an electron.

- Möller scattering: The emitted $\beta$-particle scatters in the material, ejecting a second electron.

- Compton effect: A $\beta$-particle is emitted with a de-excitation photon. This photon undergoes compton scattering, producing a second electron. 


\begin{tabular}{|c|c|c|c|c|c|c|c|c|c|c|c|c|}
\hline & \multicolumn{7}{|c|}{${ }^{238} \mathrm{U}$} & \multicolumn{5}{|c|}{${ }^{232} \mathrm{Th}$} \\
\hline U & \begin{tabular}{|c|}
$\mathrm{U}-238$ \\
$+.4710^{\circ}$ \\
$\mathrm{yc}$
\end{tabular} & & \begin{tabular}{|l|}
$\mathrm{U}-234$ \\
$2 .+510^{5}$ \\
$\mathrm{fyc}$
\end{tabular} & & & & & & & & & \\
\hline $\mathrm{Pa}$ & $\sqrt{ }$ & $\begin{array}{l}\mathrm{Pa}-234 \\
1.17 \mathrm{~m} \\
\end{array}$ & $\downarrow$ & & $\beta$ & $\not$ & & & & & & \\
\hline Th & $\begin{array}{r}T b-234 \\
2+.1 \mathrm{~d}\end{array}$ & & \begin{tabular}{|c|}
$\mathrm{h}-230$ \\
$75+\infty 0$ \\
yc
\end{tabular} & & $\alpha$ & & & $\begin{array}{c}T b-232 \\
1+10^{9} \\
y c\end{array}$ & & $\begin{array}{l}T \mathrm{~b}-228 \\
1.913 \mathrm{yr} \\
\Rightarrow\end{array}$ & & \\
\hline $\mathrm{Ac}$ & & & $\downarrow$ & & & & & $\downarrow$ & $\begin{array}{c}A c-228 \\
6.15 \mathrm{~h} \\
\not\end{array}$ & 1 & & \\
\hline $\mathrm{Ra}$ & & & \begin{tabular}{|c|}
$\mathrm{Ra}-226$ \\
$1000 \mathrm{yr}$
\end{tabular} & & & & & \begin{tabular}{|}
$\mathrm{Ra}-228$ \\
$5.75 \mathrm{yc}$
\end{tabular} & & $\begin{array}{c}\text { Ra-224 } \\
3.06 \mathrm{~d}\end{array}$ & & \\
\hline Fr & & & $\downarrow$ & & & & & & & $\downarrow$ & & \\
\hline $\mathrm{R} \pi$ & & & $\begin{array}{r}\mathrm{Rn}-222 \\
3.82+d \\
\end{array}$ & & & & & & & $\begin{array}{c}R \pi-220 \\
55.68\end{array}$ & & \\
\hline At & & & $v$ & & & & & & & $\Downarrow$ & & \\
\hline Po & & & $\begin{array}{c}\text { Eo-218 } \\
3.10 \mathrm{~m}\end{array}$ & & $\begin{array}{l}\mathrm{Po}-214 \\
16+\mu \mathrm{s} \\
\end{array}$ & & $\begin{array}{r}\mathrm{Po}-210 \\
13 \mathrm{~B}+\mathrm{d} \\
7\end{array}$ & & & $\begin{array}{c}\text { Fo-216 } \\
1+5 \mathrm{~ms}\end{array}$ & & $\begin{array}{l}\mathrm{Po}-212 \\
300 \mathrm{rs} \\
7\end{array}$ \\
\hline $\mathrm{Bi}$ & & & $\downarrow$ & $\begin{array}{l}\mathrm{Bi}-214 \\
19.9 \mathrm{~m} \\
7\end{array}$ & 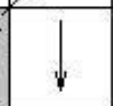 & ${ }^{\mathrm{Bi}-210}$ & $\downarrow$ & & & $\downarrow$ & $\begin{array}{l}\mathrm{Bi}-212 \\
6.5 \mathrm{~m} \\
\not\end{array}$ & 1 \\
\hline $\mathrm{Pb}$ & & & $\begin{array}{c}\mathrm{Pb}-214 \\
26.8 \mathrm{~m}\end{array}$ & 总| & $\begin{array}{l}\mathrm{Pb}-210 \\
22.3 \mathrm{yr} \\
7\end{array}$ & 1 & $\begin{array}{c}\mathrm{Pb}-206 \\
\text { stable }\end{array}$ & & & $\begin{array}{c}\mathrm{Pb}-212 \\
10.6 \mathrm{~h}\end{array}$ & : & $\begin{array}{c}\mathrm{Pb}-208 \\
\text { stable }\end{array}$ \\
\hline $\mathrm{Tl}$ & & & & $\begin{array}{c}\mathrm{Tl}-2.10 \\
1.3 \mathrm{~m}\end{array}$ & & $\begin{array}{c}\text { Tl-206 } \\
+.19 \mathrm{~m}\end{array}$ & & & & & \begin{tabular}{|c|}
$\mathrm{Tl}-208$ \\
$3.1 \mathrm{~m}$
\end{tabular} & \\
\hline
\end{tabular}

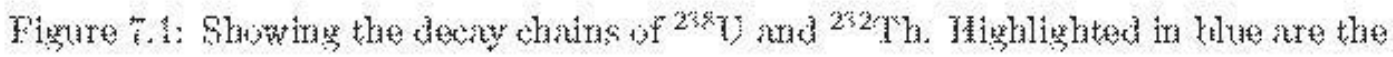

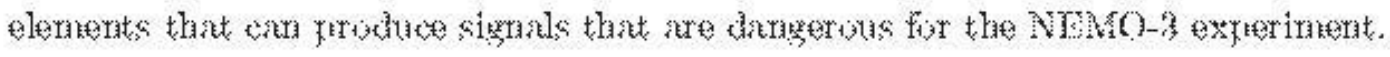

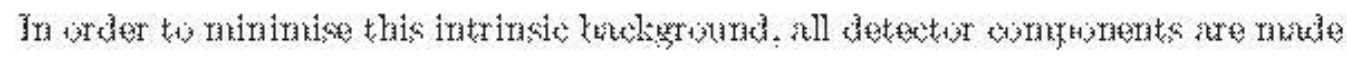

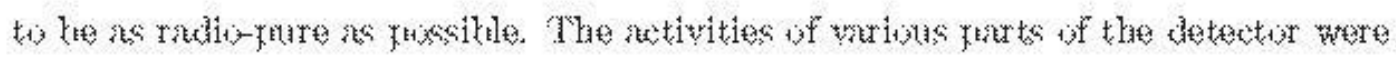

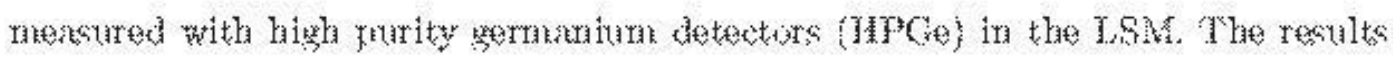

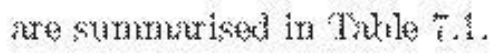

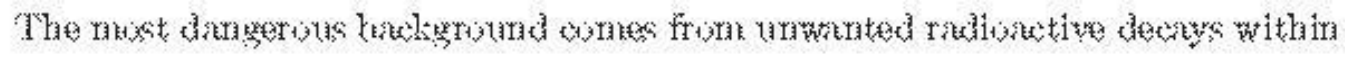

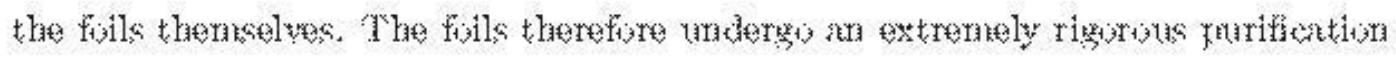

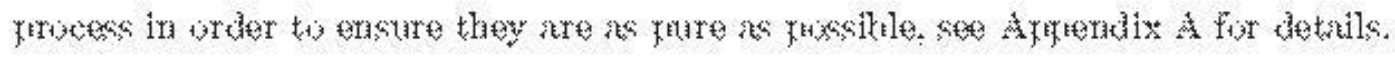

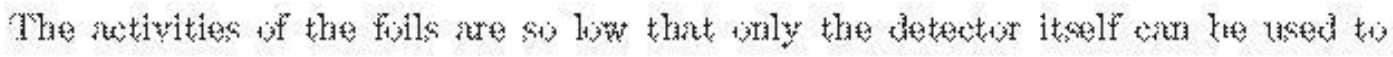
determine accurate mencureranerats. 


\begin{tabular}{|c|c|c|c|}
\hline Detector Element & Weight $(\mathrm{kg})$ & ${ }^{214} \mathbf{B i}(\mathbf{B q})$ & ${ }^{208} \mathbf{T l}(\mathbf{B q})$ \\
\hline PMTs & 600 & 300 & 18 \\
Scintillators & 5000 & $<0.7$ & $<0.3$ \\
Copper Frame & 25000 & $<25$ & $<10$ \\
Steel Frame & 10000 & $<6$ & $<8$ \\
$\mu$-metal & 2000 & $<2$ & $<2.7$ \\
Wires & 1.7 & $<10^{-3}$ & $<6 \times 10^{-4}$ \\
Iron Shield & 180000 & $<300$ & $<300$ \\
\hline
\end{tabular}

Table 7.1: The activities of the principle detector elements of NEMO-3 as determined by an HPGe detector.

\subsubsection{Simulation and Data}

All of the Monte Carlo simulation used in this work was produced using 'nemos', purpose written, Geant 3.21 based code [78]. The full NEMO-3 geometry is passed to the code, allowing for simulations of all processes of all the isoptopes present and internal and external backgrounds from any point in or around the detector. The output is reconstructed using the 'nemor' package, which is also used for reconstruction of actual data events. For the purposes of this analysis, 150,000 Monte Carlo ${ }^{208} \mathrm{Tl}$ and $150,000{ }^{214} \mathrm{Bi}$ events were generated, their source being the Molybdenum foils of the detector. This was then compared to 47 days and 23 hours of real data, taken between $01 / 05 / 2003$ and $30 / 06 / 2003$. This corresponded to 150 normal data taking runs.

\subsubsection{Particle Definitions}

An electron emitted from a source foil is defined as a reconstructed track that has fired Geiger cells near the source foils, passes through the wire chamber, has negative curvature and finally hits a scintillator on one of the detector outer walls. Several internal/external hypothesis tests are applied to events to ensure that they have arisen in the foils and travelled outwards and have not started outside of the detector and travelled inwards. Positrons have positive curvature within the detector.

Photons are seen as energy deposits in the scintillators, with no associated track. 
Apart from the case of single electron only events, the observation af a photon will typically start the data acquisition trigger. Photons are sometimes reflected from a PM, firing another one and simulating a two photon event. A time of flight hypothesis can be applied to remove photons caused by reflection.

$\alpha$-particles are also produced in the detector, for example from the decay of ${ }^{222} \mathrm{Rn}$. They are characterised by 'delayed' Geiger hits, coming some time after those produced by electrons. Hits of this type are recorded by the 'Slow TDC' counters. $\alpha$-particles are displayed as blue, square boxes in the reconstruction, to distinguish them from electrons (red circles).

\subsubsection{Selection Cuts}

A number of simple event parameters can be used to distinguish typical ${ }^{208} \mathrm{Tl}$ and ${ }^{214} \mathrm{Bi}$ decay events from $0 \nu \beta \beta$ or $2 \nu \beta \beta$ events. In both cases, a single electron track is expected with $0,1,2$ or 3 photons in addition. The energies of these photons must fall into specific windows to be considered valid background events. In the case

of ${ }^{214} \mathrm{Bi}$, an alpha-particle is also expected. The specific definitions for ${ }^{208} \mathrm{Tl}$ will be described in Section 7.2 and for ${ }^{214} \mathrm{Bi}$ in Section 7.3 .

Once a clear set of events has been established that abide by the basic expectations of that event class, care must be taken to ensure that the components of the event are associated with each other and are not part of some other, external event. This can be tested by considering the following hypotheses:

- Internal origin of electron

- External origin of photon

- Internal origin of multiple photons

- Reflection of photon

The $\chi^{2}$ value is calculated for each of these hypotheses per event and then converted to probabilities (see 7.1.8). 


\section{Internal origin of electron - $\chi_{e(i n t)}^{2}$}

Defining the length of the electron track as $\mathrm{L}_{e}$, the triggering time of an initial photon as $\mathrm{t}_{\gamma}$, the time the electron impinged upon a scintillator block as $\mathrm{t}_{e}$ and the energy deposited in that scintillator as $\mathrm{E}_{e}$, the electron's time of flight can be determined by:

$$
\Delta t_{e}=\frac{L_{e}}{\beta_{e} c}
$$

where $c$ is the speed of light in a vacuum and $\beta_{e}$ is given by:

$$
\beta_{e}=\frac{\sqrt{E_{e}\left(E_{e}+2 m_{e} c^{2}\right)}}{E_{e}+m_{e} c^{2}}
$$

where $m_{e}$ is the mass of the electron. The emission time of all the particles should then be:

$$
t_{\text {emission }}=t_{e}-\frac{L_{e}}{\beta_{e} c}=t_{\text {gamma }}
$$

and the $\chi_{e(\text { int })}^{2}$ variable can be expressed as:

$$
\chi_{e(i n t)}^{2}=\frac{\left(t_{\gamma}-\left(t_{e}-\frac{L_{\varepsilon}}{\beta_{\varepsilon} c}\right)\right)^{2}}{\sigma_{t o t}^{2}}
$$

where $\sigma_{\text {tot }}^{2}$ is the total of all the errors related to the time measurement of the event and to the calculation of $t_{e}$ :

$$
\sigma_{t o t}^{2}=\sigma_{t_{i}}^{2}+\sigma_{t_{0}}^{2}+\sigma_{L_{(i)}}^{2}
$$

where $\sigma_{L_{(i)}}^{2}$ is given by:

$$
\sigma_{L_{(i)}}^{2}=\left(\frac{1}{c L}\right)^{2}\left(\begin{array}{c}
\left(x_{i}-x_{0}\right)^{2}\left(\sigma_{x_{i}}^{2}+\sigma_{x_{0}}^{2}\right)+ \\
\left(y_{i}-y_{0}\right)^{2}\left(\sigma_{y_{i}}^{2}+\sigma_{y_{0}}^{2}\right)+ \\
\left(z_{i}-z_{0}\right)^{2}\left(\sigma_{z_{i}}^{2}+\sigma_{z_{0}}^{2}\right)
\end{array}\right)
$$

the (i) here denotes the particle type in question in the event. The probability distribution is shown in Figure 7.2. 


\section{External origin of photon(s) $-\chi_{\gamma(e x t)}^{2}$}

We cannot completely exclude background events triggered by a photon coming from a source external to the detector and subsequently producing an electron by the Compton effect. In a similar way to that previously shown, a $\chi_{\gamma(e x t)}^{2}$ variable can be constructed as follows:

$$
\chi_{\gamma(e x t)}^{2}=\frac{\left(t_{e}-\left(t_{\gamma}+\frac{L_{\gamma}}{c}\right)\right)^{2}}{\sigma_{t o t}^{2}}
$$

The probability distribution is plotted in Figure 7.3.

\section{Internal origin of 2 or more photons $-\chi_{\gamma \gamma(i n t)}^{2}$}

In the case that there are multiple photons in the event, the following variable can be found, to ensure that all the event components are consistent with each other in time:

$$
\chi_{\gamma \gamma(i n t)}^{2}=\frac{\left(t_{\gamma 1}-\left(t_{e}-\frac{L_{\gamma 1}}{c}\right)\right)^{2}}{\sigma_{t o t}^{2}}+\frac{\left(t_{\gamma 2}-\left(t_{e}-\frac{L_{\gamma 2}}{c}\right)\right)^{2}}{\sigma_{t o t}^{2}}
$$

Figure 7.4 shows the resulting distribution.

\section{Reflection of photon $-\chi_{\gamma \gamma(\text { ref })}^{2}$}

The final possibility to consider is that a 2 photon event in fact was caused by just one photon and a reflection from a scintillator face. In this case, the timing of one photon is more consistent with having come from a scintillator than the foil. The $\chi_{\gamma \gamma(\text { ref })}^{2}$ variable has the form:

$$
\chi_{\gamma \gamma(r e f)}^{2}=\frac{\left(t_{\gamma 1}-\left(t_{e}-\frac{L_{\gamma 1}}{c}\right)\right)^{2}}{\sigma_{t o t}^{2}}+\frac{\left(t_{\gamma 2}-\left(t_{e}-\frac{L_{\gamma 1 \gamma 2}}{c}\right)\right)^{2}}{\sigma_{t o t}^{2}}
$$

where $\mathrm{L}_{\gamma 1 \gamma_{2}}$ is the distance between the scintillators that were hit by the photons. This probability distribution is shown in Figure 7.5. 


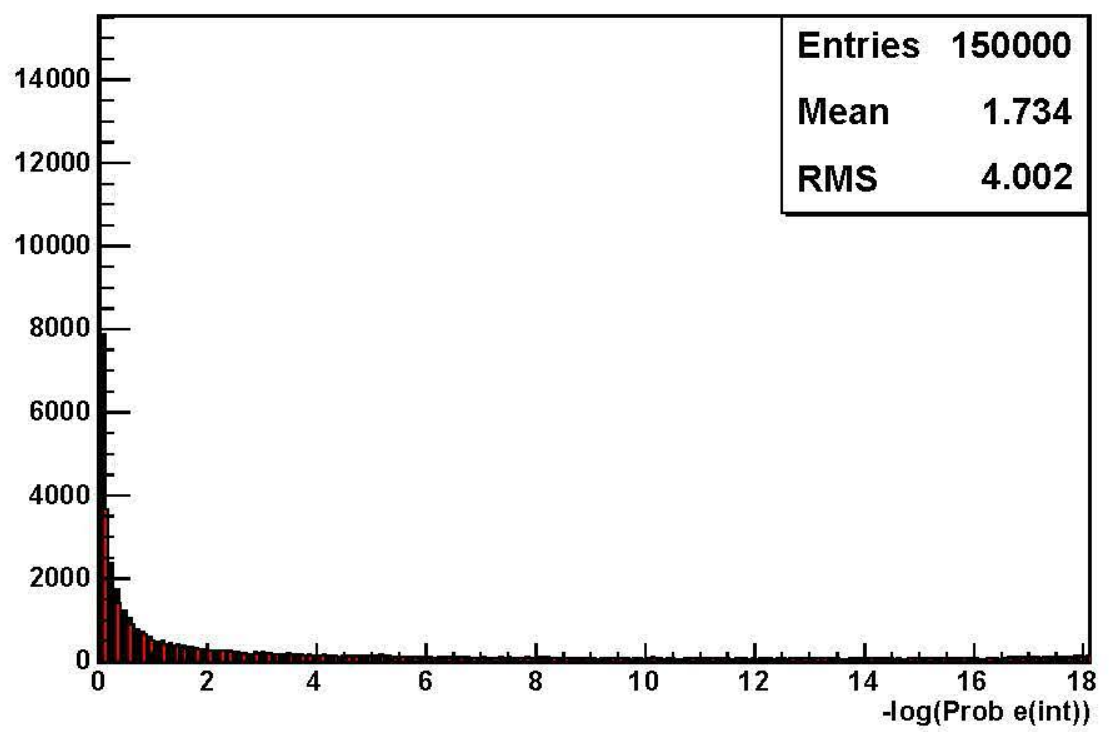

Figure 7.2: Showing the probability distribution for the hypothesis that the electron in the event has the same common source as any photons.

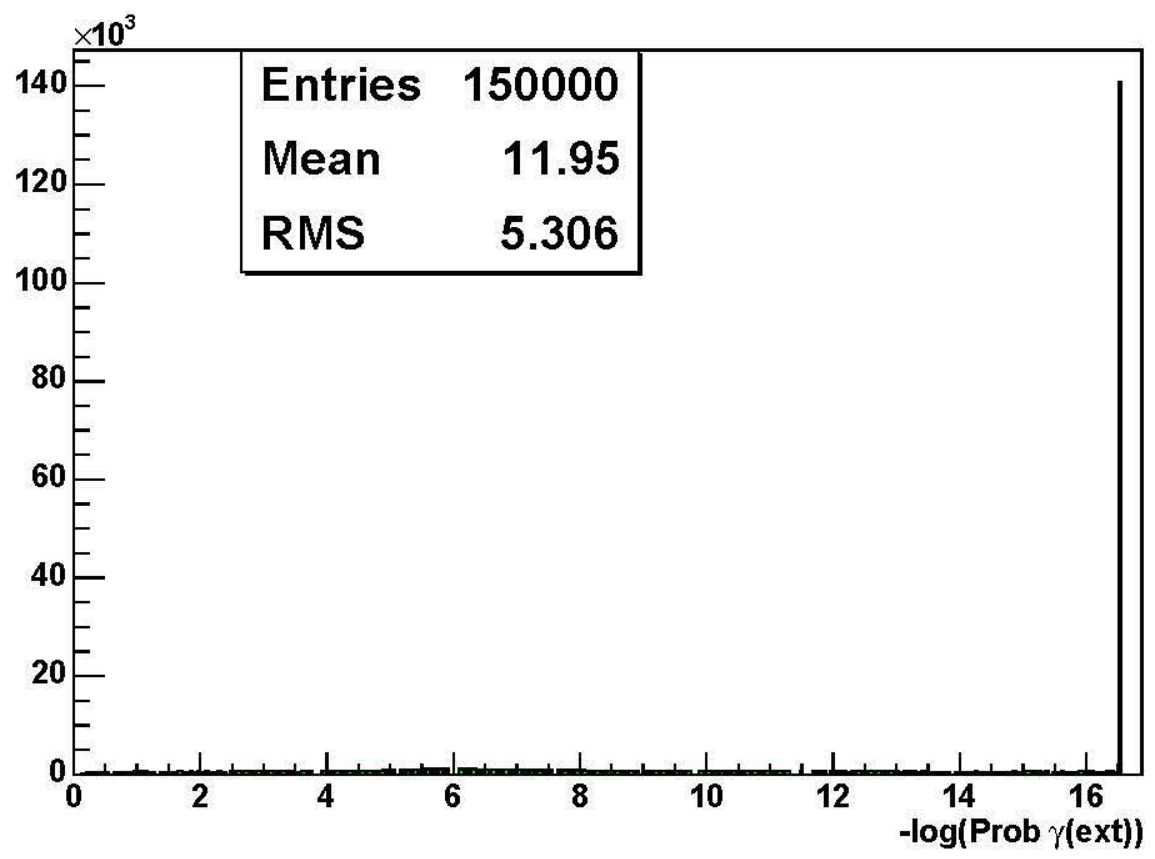

Figure 7.3: Showing the probability distribution for the hypothesis that a photon does not have the same common source as the electron. All events with $\log (\operatorname{Prob} \gamma($ ext $)>16.5$ are shown together. 


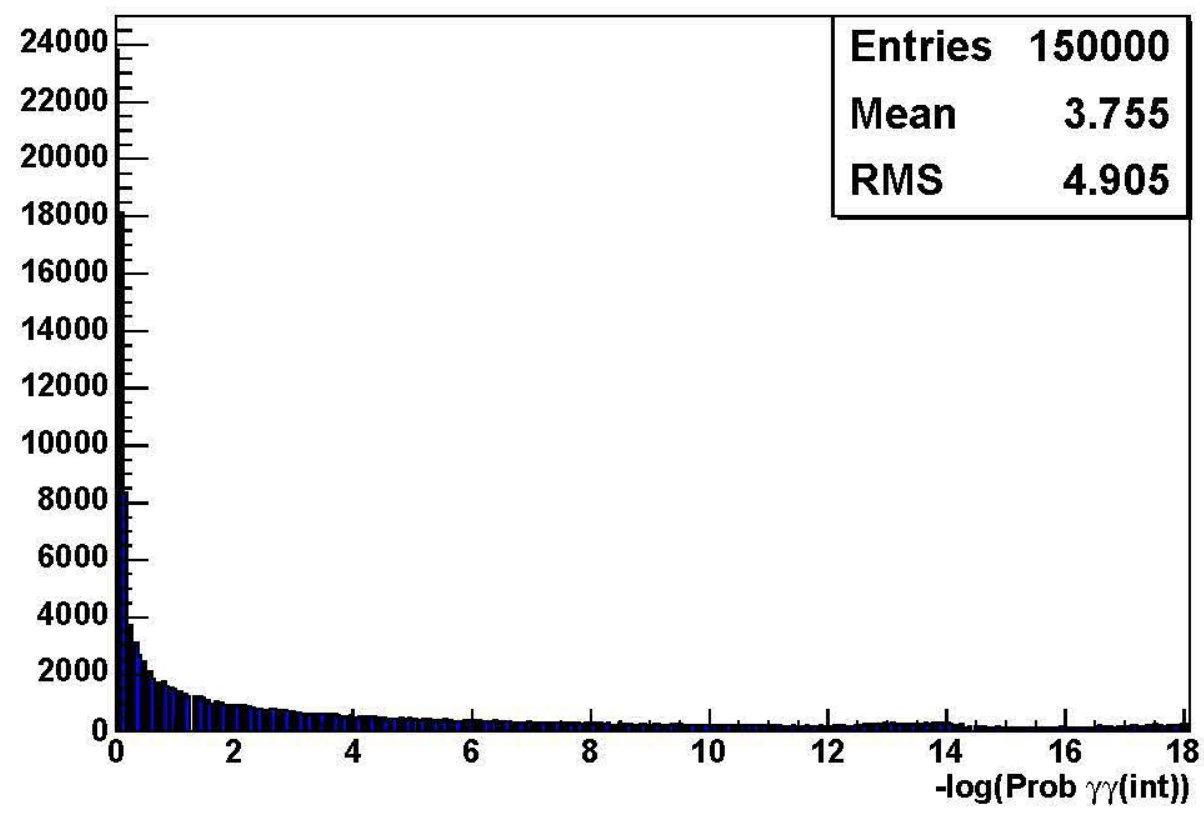

Figure 7.4: Showing the probability distribution for the hypothesis that two separate photons in the event have the same common source as the electron.

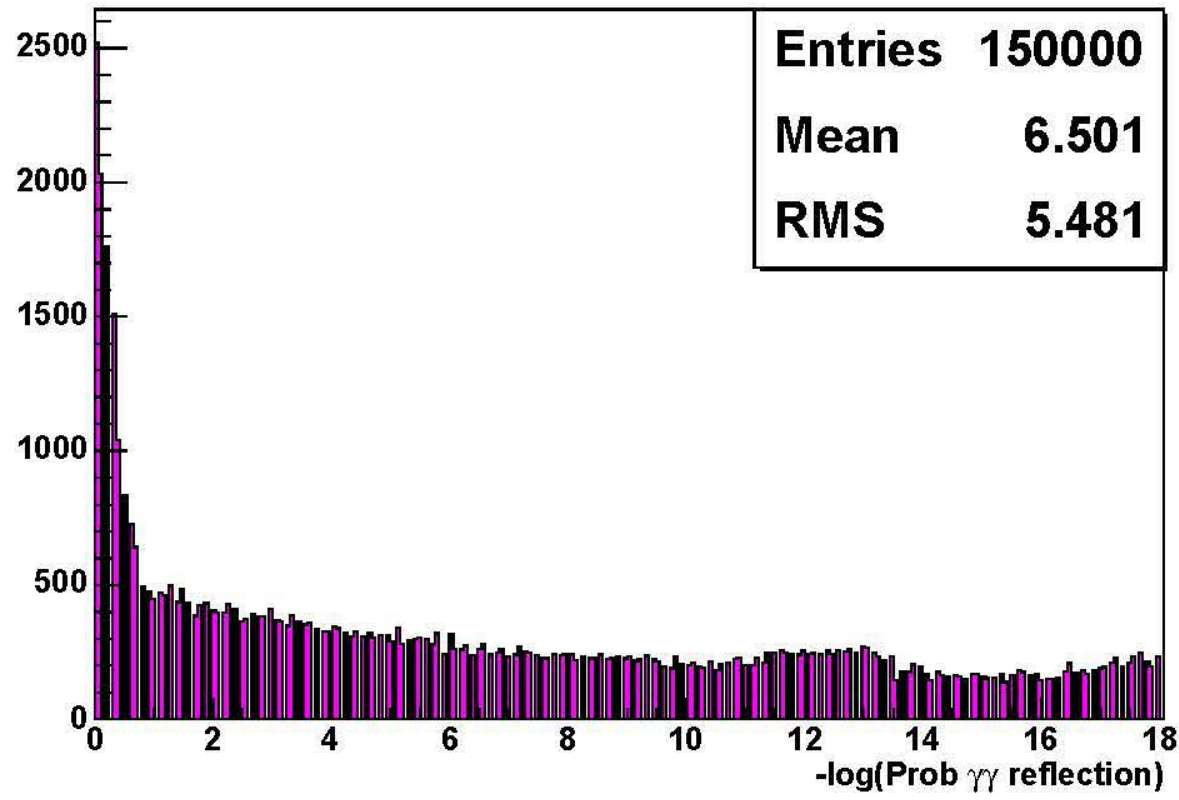

Figure 7.5: Showing the probability distribution for the hypothesis that a photon has been reflected rather then there being two separate photons in the event. 


\subsubsection{Calculation of Probability from $\chi^{2}$}

The probability density function for the $\chi^{2}$ distribution with $\mathrm{r}$ degrees of freedom is given by:

$$
P_{r}(x)=\frac{x^{r / 2-1} e^{-x / 2}}{\Gamma\left(\frac{1}{2} r\right) 2^{r / 2}}
$$

For $\mathrm{x} \in(0, \infty), \Gamma(\mathrm{x})$ is a gamma function given by:

$$
\Gamma_{x}=\int_{0}^{\infty} t^{x-1} e^{-t} d t
$$

and the cumulative distribution function and the probability, $Q$ is given by:

$$
\operatorname{Qr}\left(\chi^{2}\right)=\int_{0}^{\chi^{2}} \frac{t^{r / 2-1} e^{-t / 2} d t}{\Gamma\left(\frac{1}{2} r\right) 2^{r / 2}}
$$

\subsubsection{Calculation of Number of Events}

The number of observed events can be derived from the radioactive decay law:

$$
N_{e, n t}(t)=\eta N_{\text {atoms }}\left(1-e^{-\ln 2 \frac{t}{T_{1 / 2}}}\right)
$$

where $\eta$ is the detection efficiency of the channel in question, $\mathrm{N}_{\text {atoms }}$ is the number of atoms in the sample, $t$ is the net data acquisition time and $T_{1 / 2}$ is the half-life of the decay mode being studied. For rare events, this simplifies to:

$$
N_{e \eta t}(t)=\eta N_{a v} \frac{M}{m_{a t}} \ln 2 \frac{t}{T_{1 / 2}}
$$

where $\mathrm{N}_{a v}$ is Avagadro's number, $\mathrm{M}$ is the mass of the considered sample and $\mathrm{m}_{a t}$ is the atomic mass of that sample.

In the case of a radioactive source of known activity, $\mathrm{A}(\mathrm{Bq} / \mathrm{kg})$, the number of events is given by:

$$
N_{e v t}(t)=\eta A M t
$$




\subsection{Measurement of the ${ }^{208} \mathrm{Tl}$ Background in the Foils}

\subsubsection{Method}

By making a measurement of the quantity of ${ }^{208} \mathrm{Tl}$ contamination of the foils, an estimate can be made of the number of events that are expected to mimic $0 \nu \beta \beta$ decays. The first step is to identify events that are characteristic of ${ }^{208} \mathrm{Tl}$ only. Using a chart of major decay transitions such as that shown in Figure 7.6, it is possible to isolate specific event quantities that can pinpoint ${ }^{208} \mathrm{Tl}$ decays. Three event types are studied: those with a single electron detected and one, two or three photons in accompaniment. Since the total energy of the transition is $4.99 \mathrm{MeV}$, this leads to the event profiles shown in Table 7.2 .

\begin{tabular}{|c|c|c|c|c|}
\hline $\mathrm{e}^{-} \mathbf{E}_{\max }$ & $\mathbf{E}_{\gamma 1}$ & $\mathbf{E}_{\gamma 2}$ & $\mathbf{E}_{\gamma 3}$ & Probability \\
\hline 2.375 & 2.615 & & & $0.03 \%$ \\
\hline 1.792 & 2.615 & 0.583 & & $(0.51 \times 0.87)=44 \%$ \\
1.515 & 2.615 & 0.860 & & $(0.217 \times 0.12)=0.026 \%$ \\
\hline 1.515 & 2.615 & 0.583 & 0.277 & $(0.217 \times 0.064 \times 0.87)=0.01 \%$ \\
1.281 & 2.615 & 0.583 & 0.511 & $(0.228 \times 0.22 \times 0.87)=0.04 \%$ \\
1.029 & 2.615 & 0.583 & 0.763 & $(0.031 \times 0.016 \times 0.87)=0.000426 \%$ \\
\hline
\end{tabular}

Table 7.2: The maximum electron energies and the decay photon energies for various ${ }^{208} \mathrm{Tl}$ event types. The energies shown are in $\mathrm{MeV}$.

\section{Description of Cuts}

An event is classed as being a ${ }^{208} \mathrm{Tl}$ decay if it satisfies the following criteria:

- 1 charged particle track only: $\operatorname{In}^{208} \mathrm{Tl}$ decays, in the channel being studied only one electron per event should be produced. Events with more than 1 track are disgarded. Noise hits or overlapping events can potentially generate additional tracks in a genuine decay. This scenario cannot be separated from more complicated events, however. 


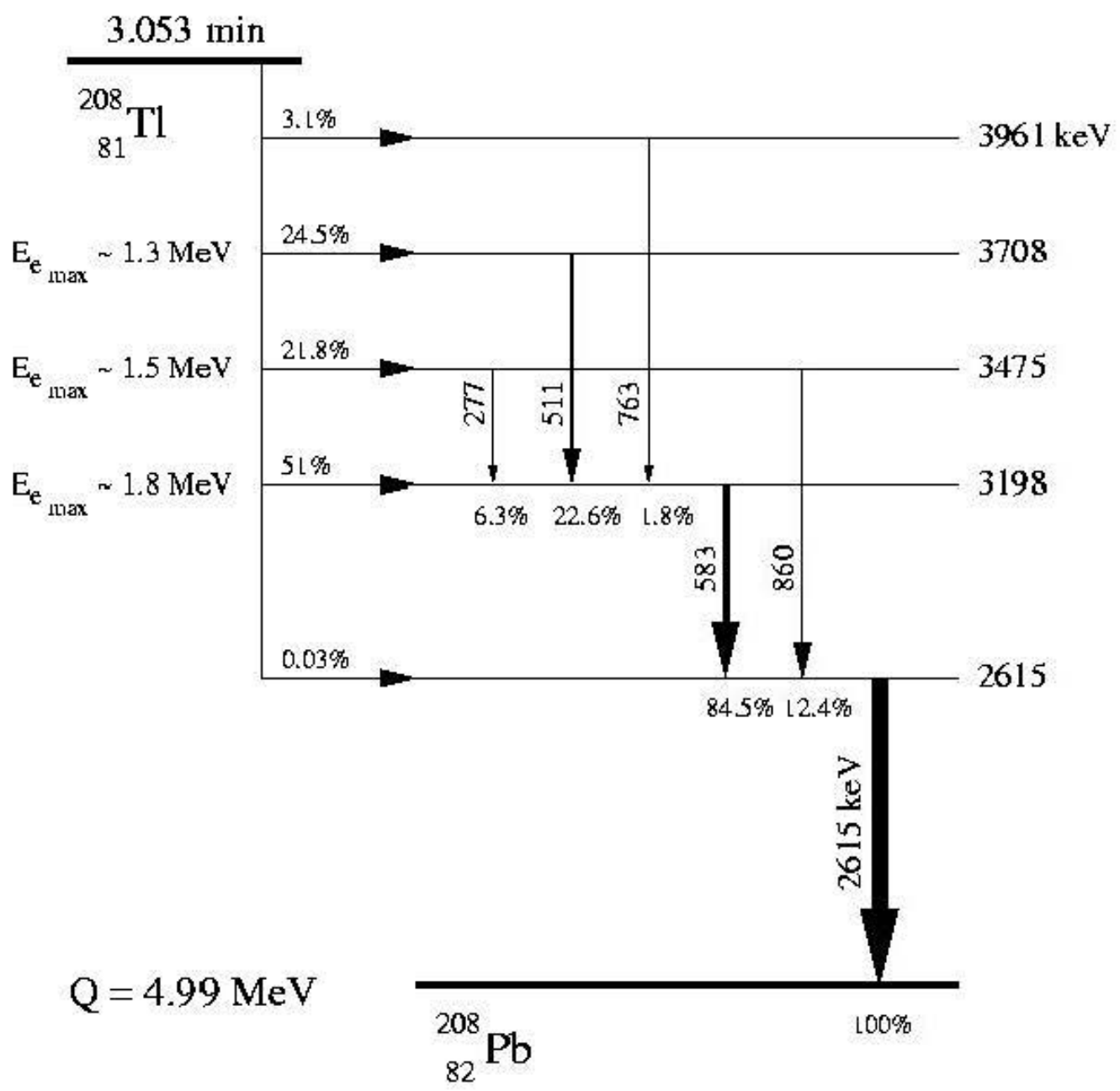

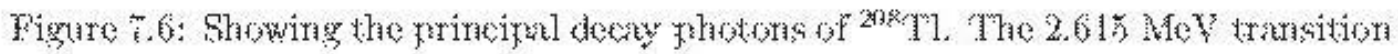
always ocetro.

- Charged particle hit scintillator: A meacurement of the energy of the

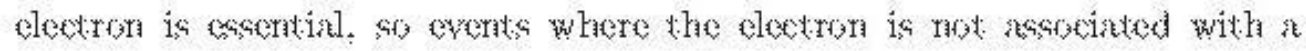
secirtillatior matsist he remowed.

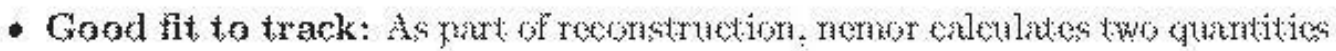

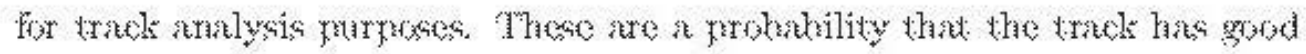

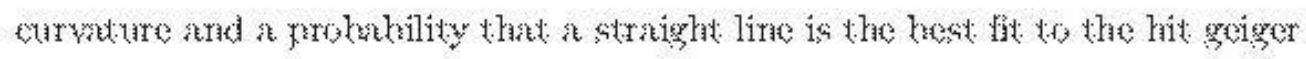

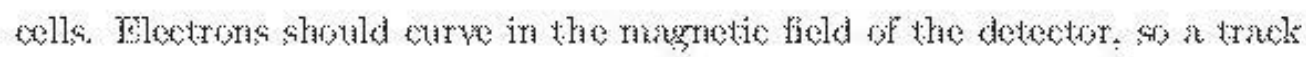

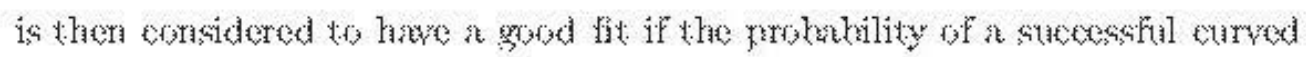


fit $>0.1$ and if the probability of a line fit is $<0.9$.

- Curvature of track suggest electron: Events that are more consistent with being positrons, or have no charge assigned at all are removed.

- At least one hit in row closest to Mo foil: Only the contamination of the Molybdenum foils is being considered, so only events in the appropriate sectors are passed. Also, to reduce the chance of an electron entering the detector from outside or some other unrelated internal source, there must be at least one hit in the row of Geiger cells closest to the foil.

- $0.5<$ Electron energy $<1.3$ : Events with electron energies outside this region (in $\mathrm{MeV}$ ) are not consistent, with having resulted from a ${ }^{208} \mathrm{Tl}$ decay.

- $\chi_{e(i n t)}^{2}<2$ : The internal hypothesis for the electron must be strongly supported for the event to pass.

- $\chi_{\gamma(e x t)}^{2}>3$ : Only events where it is unlikely that a photon originated other than from the event itself, are considered.

- $\chi_{\gamma \gamma(\text { int })}^{2}<2:$ In the case of there being more than one photon, there should be a strong likelihood that they originated from the same source and consistent with that source being the same as the electron.

- $\chi_{\gamma \gamma(r e f)}^{2}>3$ : The probability that a photon scattered and was subsequently re-detected should be low. 

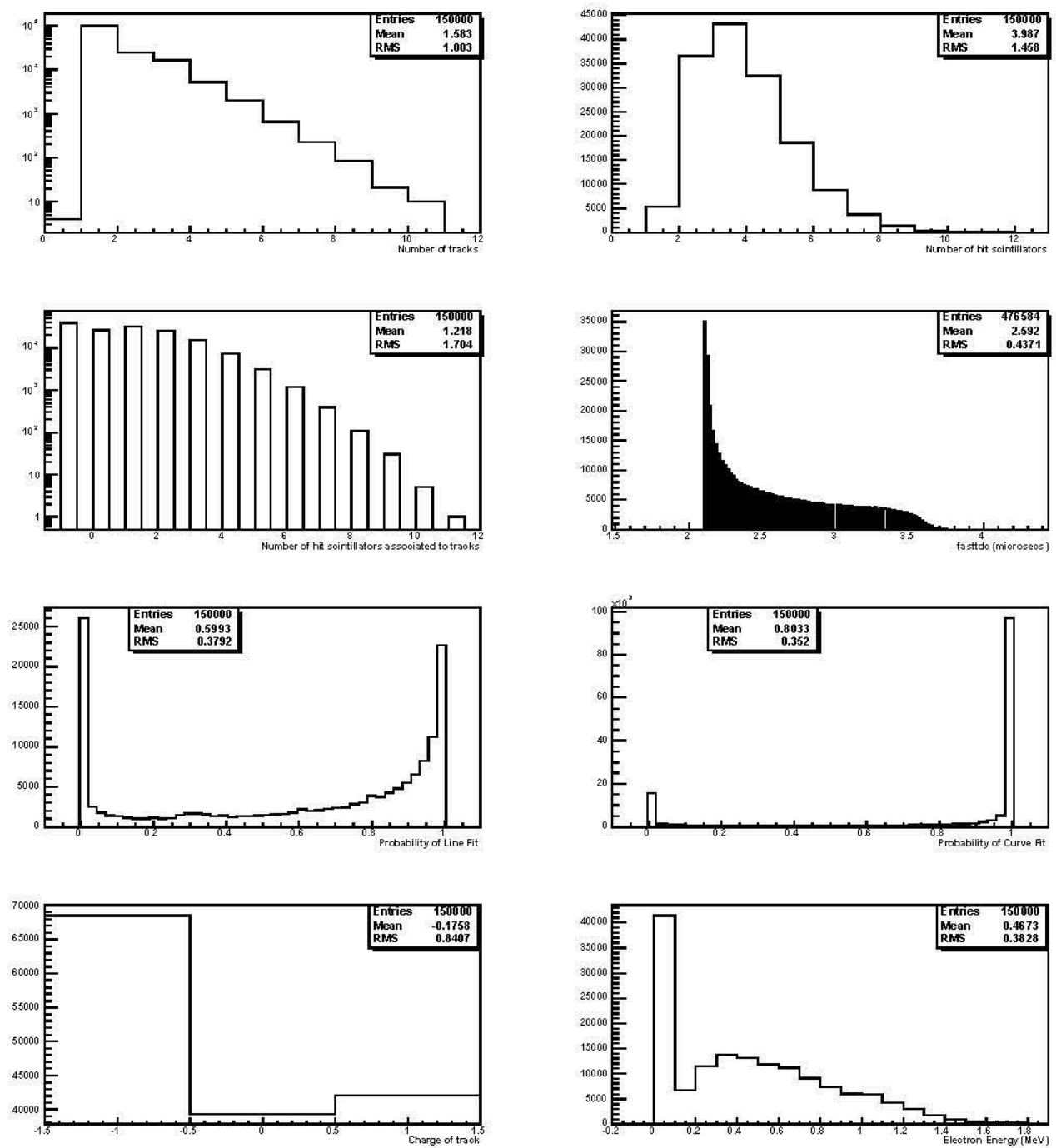

Figure 7.7: Showing the principle events quantities associated with ${ }^{208} \mathrm{Tl}$ Monte Carlo decays.

A large amount of Monte Carlo simulations of ${ }^{208} \mathrm{Tl}$ decays in foils are then produced. Analysis code is written to select out events that correspond to the above criteria. This results in an efficiency factor which is used later. This factor represents how well $\mathrm{Tl}$ events can be found based on the analysis cuts and is expected to be fairly low since only a portion of all types of $\mathrm{Tl}$ decays are considered and more importantly, only perfect ones are; many events may contain noise, contamination or mis-identification and are discarded. 


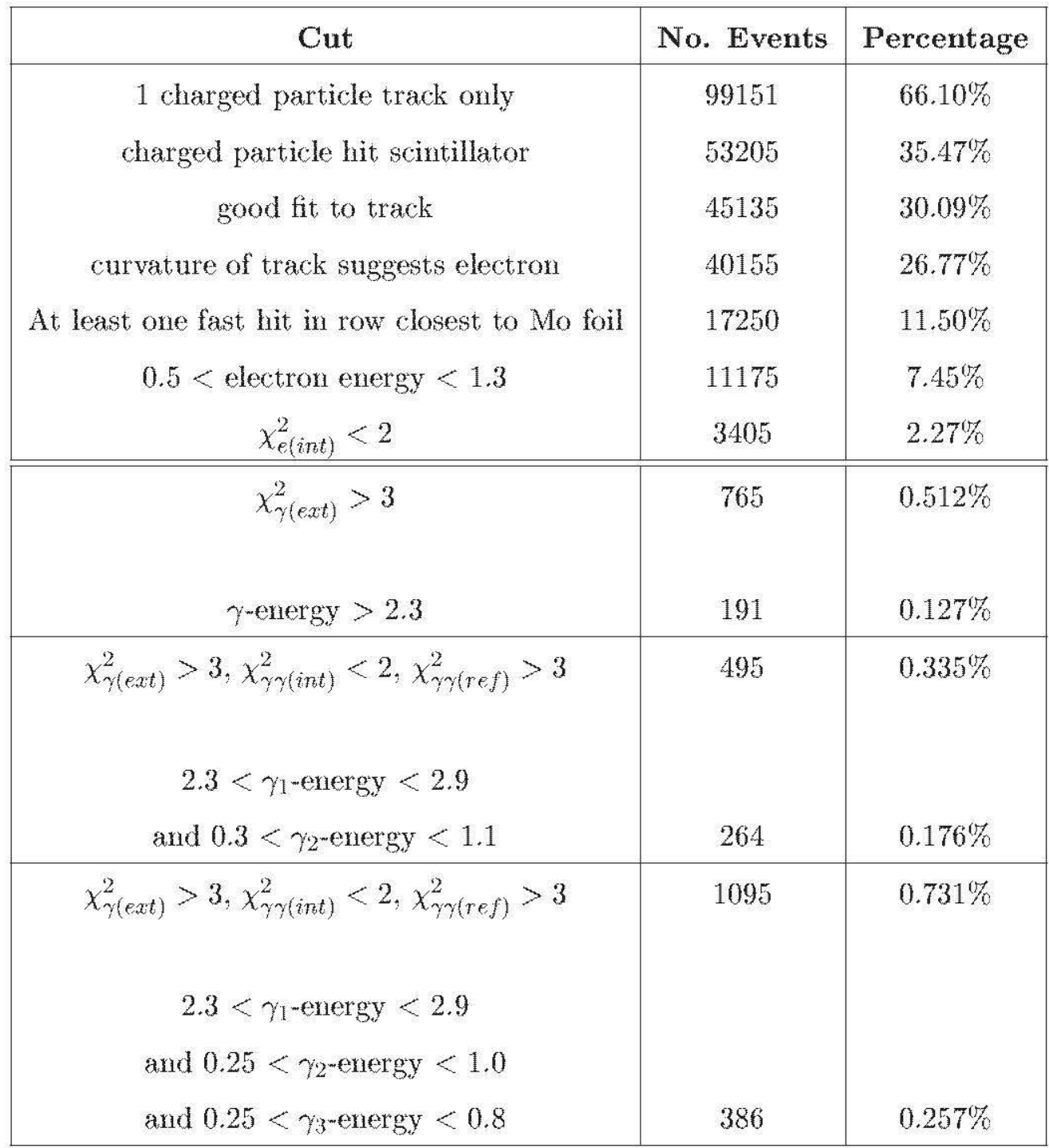

Table 7.3: Showing the selection cuts applied to the ${ }^{208} \mathrm{Tl}$ Monte Carlo dataset. The efficiencies for selection in the 1,2 and 3 photon channels are shown.

\subsubsection{Result}

Once all the described cuts have been applied to the ${ }^{208} \mathrm{Tl}$ Monte Carlo sample, there were 191, 264 and 386 events remaining in the 1,2 and 3 photon channels respectively. The sample size was originally 150,000 events, which therefore corresponds to detection efficiencies of $0.127 \%, 0.176 \%$ and $0.257 \%$ respectively.

Exactly the same procedure is then applied to the 1151 hour dataset. The upper limit, b, at $90 \%$ C.L. can then be solved for using the formula: 

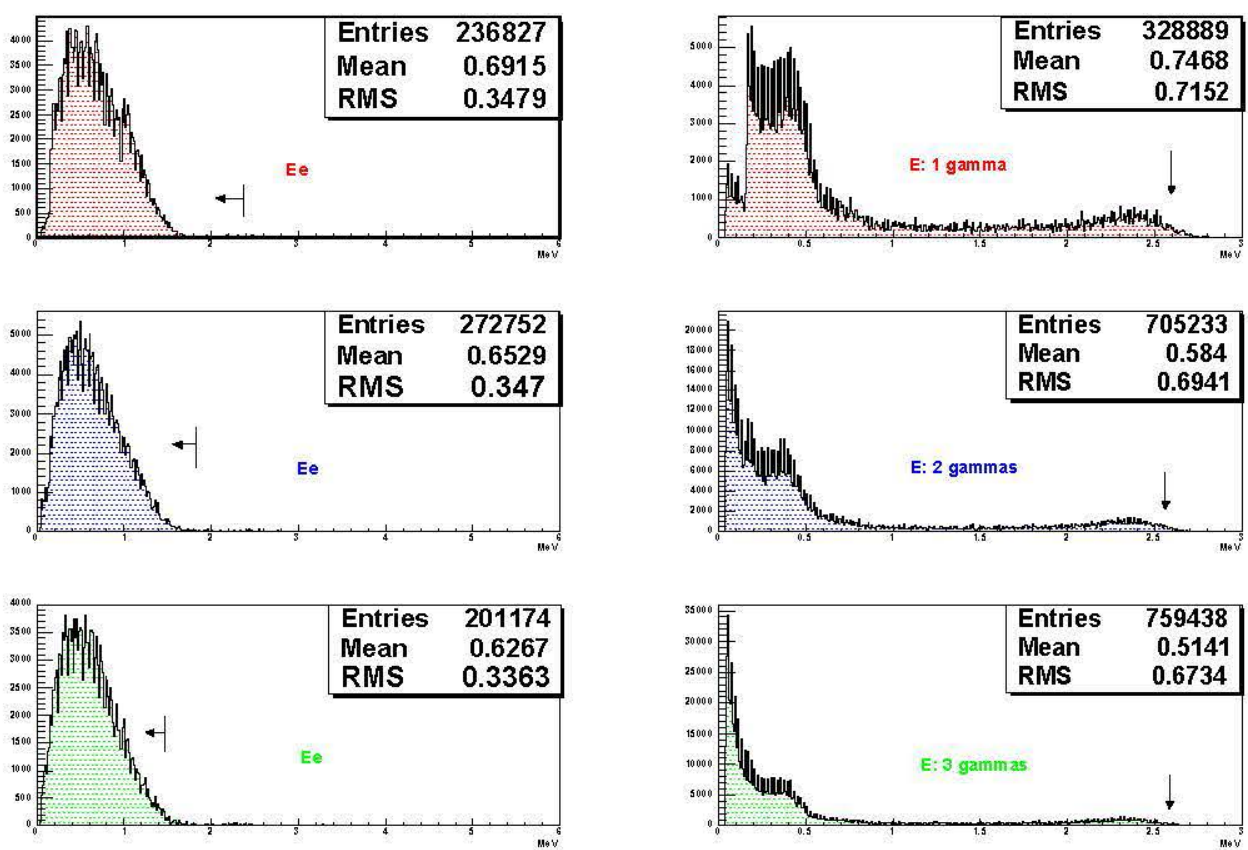

Figure 7.8: Showing the electron energies (left) with the most probable 1,2 and 3 photons transitions accompanying (right). ${ }^{208} \mathrm{Tl}$ events can be selected if the photons fall into the correct energy windows.

$$
\beta(90 \% \text { C.L. })=0.1=\sum_{n=0}^{n_{\text {obserefi }}} \frac{b^{n} e^{-b}}{n !}
$$

The results are shown in Table 7.4 .

\begin{tabular}{|c|c|c|}
\hline Channel & No. Events & Activity \\
\hline $1 \gamma$ & 0 & $<105.50 \mu \mathrm{Bq}$ \\
$2 \gamma$ & 1 & $<129.11 \mu \mathrm{Bq}$ \\
$3 \gamma$ & 2 & $<121.08 \mu \mathrm{Bq}$ \\
\hline Combined & 3 & $<69.72 \mu \mathrm{Bq}$ \\
\hline
\end{tabular}

Table 7.4: Showing the calculated activites in the the 1,2 and $3 \gamma^{208} \mathrm{Tl}$ Channel. 


\subsection{Measurement of the ${ }^{214} \mathrm{Bi}$ Background in the Foils}

\subsubsection{Method}

A measmement can be made of the anount of ${ }^{214} \mathrm{Bi}$ in the source foils in a similar way to the study of ${ }^{208}$ TI. There are mamy more possible decay tramsitions for Bismuth (See Figure 7.11), but a crucial difference is that these decays are always followed by the emission of an $\alpha$-particle in the secondary decay:

${ }^{214} \mathrm{Bi} \rightarrow{ }^{214} \mathrm{P} \xi+\beta \rightarrow{ }^{210} \mathrm{~Pb}+\alpha(164 \mu \mathrm{s} \mathrm{later})$

e-particles can be detected by the 'slow-tde' comters up to 710 fusecs after the initial geiger cell hit. When hits of this type are reconstructed by the Nemo software, they show up in event displays as blue scuares rather than the momal red circles. Figrure 7.9 shows an example of a reconstructed Monte Carlo ${ }^{214}$ Bi decay.

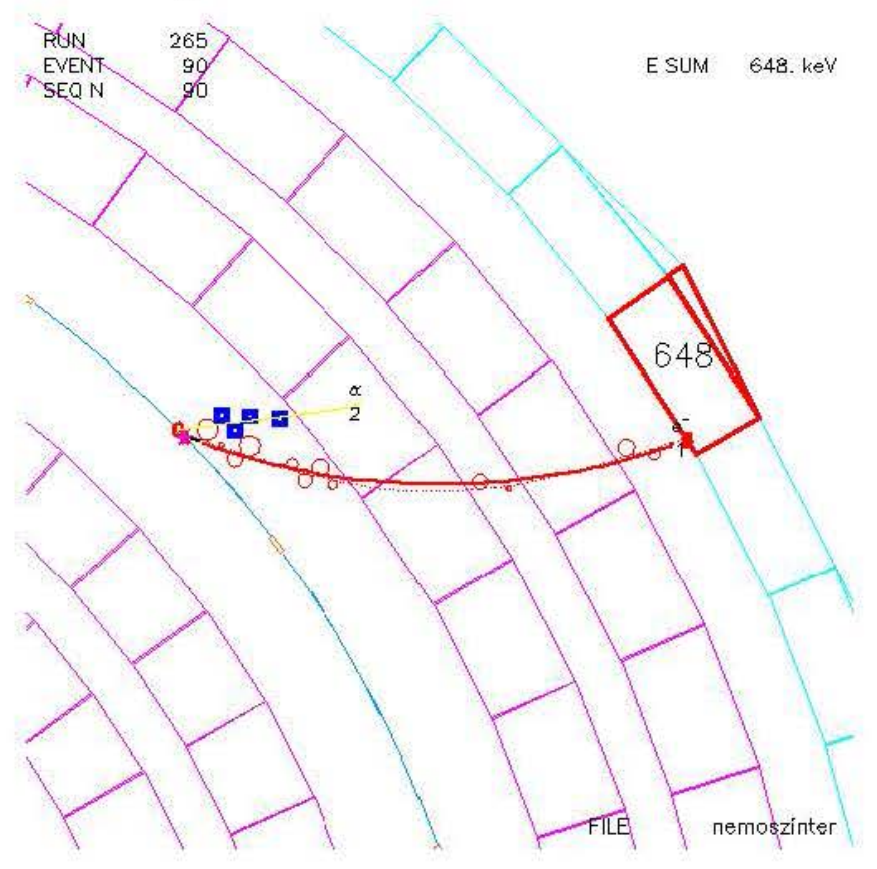

Figne 7.9: Showing a typical bismuth decay event display. The red track is the reconstructed electron path, the red box is the hit scintillator with the energy deposited by the electron shown and the bhe scuares show the delayed geiger hits camed by the alpha particle.

The maximum electron energies and the decay photon energies for various ${ }^{214} \mathrm{Bi}$ decays are shown in Table 7.5. However, since the probability of single electron 


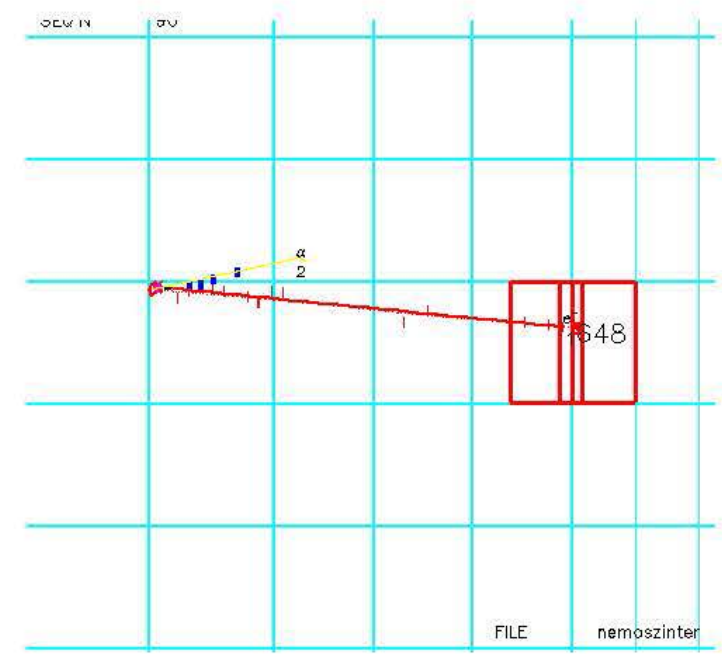

Figure 7.10: Side view of a ${ }^{214}$ Bi decay event.

events is high, only events with one electron and no associated photons are considered.

- 1 charged particle track only: See 7.2 .1

- 1 scintillator hit only: Only 1 scintillator was hit in the entire event. This ought to have been caused by the electron.

- Charged particle hit scintillator: See 7.2 .1

- Good fit to track: See 7.2.1

- Curvature of track suggests electron: See 7.2 .1

- At least one delayed hit in event: $A$ delayed hit is indicative of a ${ }^{214} \mathrm{Bi}$ decay. At least one such hit is required to pass the event.

- At least one delayed hit in row closest to Mo foil: To ensure that the delayed hit has arisen from a ${ }^{214} \mathrm{Bi}$ decay in the Molybdenum foils and not some external source, there is a requirement that one of the delayed hits is in the first row.

- At least one fast hit in row closest to Mo foil: See 7.2.1

- Delayed hit and fast hit in the same sector: The $\alpha$-particle (delayed) and electron (fast) should have originated from the same source. 


\begin{tabular}{|c|c|c|c|}
\hline $\mathrm{e}^{-} \mathbf{E}_{\max }$ & $\mathbf{E}_{\gamma 1}$ & $\mathbf{E}_{\gamma 2}$ & Probability \\
\hline 3.270 & & & $18 \%$ \\
\hline 2.661 & 0.609 & & $(0.01 \times 0.46)=0.46 \%$ \\
1.892 & 1.378 & & $(0.076 \times 0.034)=0.26 \%$ \\
1.540 & 1.730 & & $(0.18 \times 0.029)=0.52 \%$ \\
1.506 & 1.764 & & $(0.18 \times 0.158)=2.8 \%$ \\
1.423 & 1.847 & & $(0.083 \times 0.021)=0.17 \%$ \\
1.066 & 2.204 & & $(0.055 \times 0.05)=0.275 \%$ \\
0.823 & 2.447 & & $(0.028 \times 0.015)=0.042 \%$ \\
\hline 1.893 & 0.609 & 0.768 & $(0.076 \times 0.049 \times 0.46)=0.17 \%$ \\
1.727 & 0.609 & 0.934 & $(0.033 \times 0.032 \times 0.46)=0.05 \%$ \\
1.541 & 0.609 & 1.120 & $(0.18 \times 0.15 \times 0.46)=1.2 \%$ \\
1.423 & 0.609 & 1.238 & $(0.083 \times 0.059 \times 0.46)=0.23 \%$ \\
1.253 & 0.609 & 1.408 & $(0.025 \times 0.025 \times 0.46)=0.03 \%$ \\
1.152 & 0.609 & 1.509 & $(0.043 \times 0.022 \times 0.46)=0.04 \%$ \\
\hline
\end{tabular}

Table 7.5: The maximum electron energies and the decay photon energies for various ${ }^{214} \mathrm{Bi}$ decays. The energies shown are in $\mathrm{MeV}$.

- Delayed hit and fast hit in the same direction: $\alpha$-particles typically travel only a short distance and frequently do not even penetrate the surface of a foil. The chance that an electron or an alpha-particle, associated with the same event, will penetrate all the way through the foil, is very low.

- $\chi_{e(i n t)}^{2}<2:$ See 7.2 .1

\subsubsection{Result}

Having arrived at a ${ }^{214} \mathrm{Bi}$ decay detection efficiency from the analysis cuts on the Monte Carlo, application of these cuts to the data should yield the activity within the detector. The formula in this case is largely based on the identification of a $\alpha$ particles; it lends some confidence to the analysis that the half-life of the Polonium decay that produces the $\alpha$-particle can be reconstructed. Figure 7.14 shows the time 


\begin{tabular}{|c|c|c|}
\hline Cut & No. Events & Percentage \\
\hline 1 charged particle track only & 66898 & $44.60 \%$ \\
1 scintillator hit only & 25030 & $16.68 \%$ \\
charged particle hit scintillator & 18151 & $12.10 \%$ \\
good fit to track & 15014 & $10.09 \%$ \\
curvature of track suggests electron & 8558 & $5.70 \%$ \\
At least one delayed hit & 6210 & $4.14 \%$ \\
At least one delayed hit in row closest to Mo foil & 5610 & $3.74 \%$ \\
At least one fast hit in row closest to Mo foil & 1974 & $1.32 \%$ \\
Delayed hit and fast hit in same sector & 1053 & $0.70 \%$ \\
Delayed hit and fast hit in same direction & 470 & $0.31 \%$ \\
$\chi_{e(\text { int })}^{2}<$ & 256 & $0.17 \%$ \\
\hline
\end{tabular}

Table 7.6: The selection cuts applied to the ${ }^{214} \mathrm{Bi}$ Monte Carlo. The analysis began with 150,000 events.

after the initial hit that the $\alpha$-particle was detected. Here the exponential fit gives: $\mathrm{p} 1=\mathrm{T}=$ average lifetime $=\mathrm{T}^{\frac{1}{2}} / \ln 2$.

Finally, the analysis is run over the 1151 hour dataset and the upper limit on the activity calculated as before.

\begin{tabular}{|c|c|c|}
\hline Channel & No. Events & Activity (90\% C.L.) \\
\hline${ }^{214} \mathrm{Bi}$ & 4 & $<274.50 \mu \mathrm{Bq} / \mathrm{kg}$ \\
\hline
\end{tabular}

Table 7.7: Showing the calculated activity for ${ }^{214} \mathrm{Bi}$.

\subsection{Summary}

Neutrinoless double beta decay is a rare decay. All backgrounds to this decay must be studied and removed in order to ensure a good measurement can be made. Such backgrounds include the tail of the $2 \nu \beta \beta$ distribution, the interactions of external neutrons and photons with the detector and natural radioactivity of materials used for the construction of the detector, within the LSM and the source foils. 


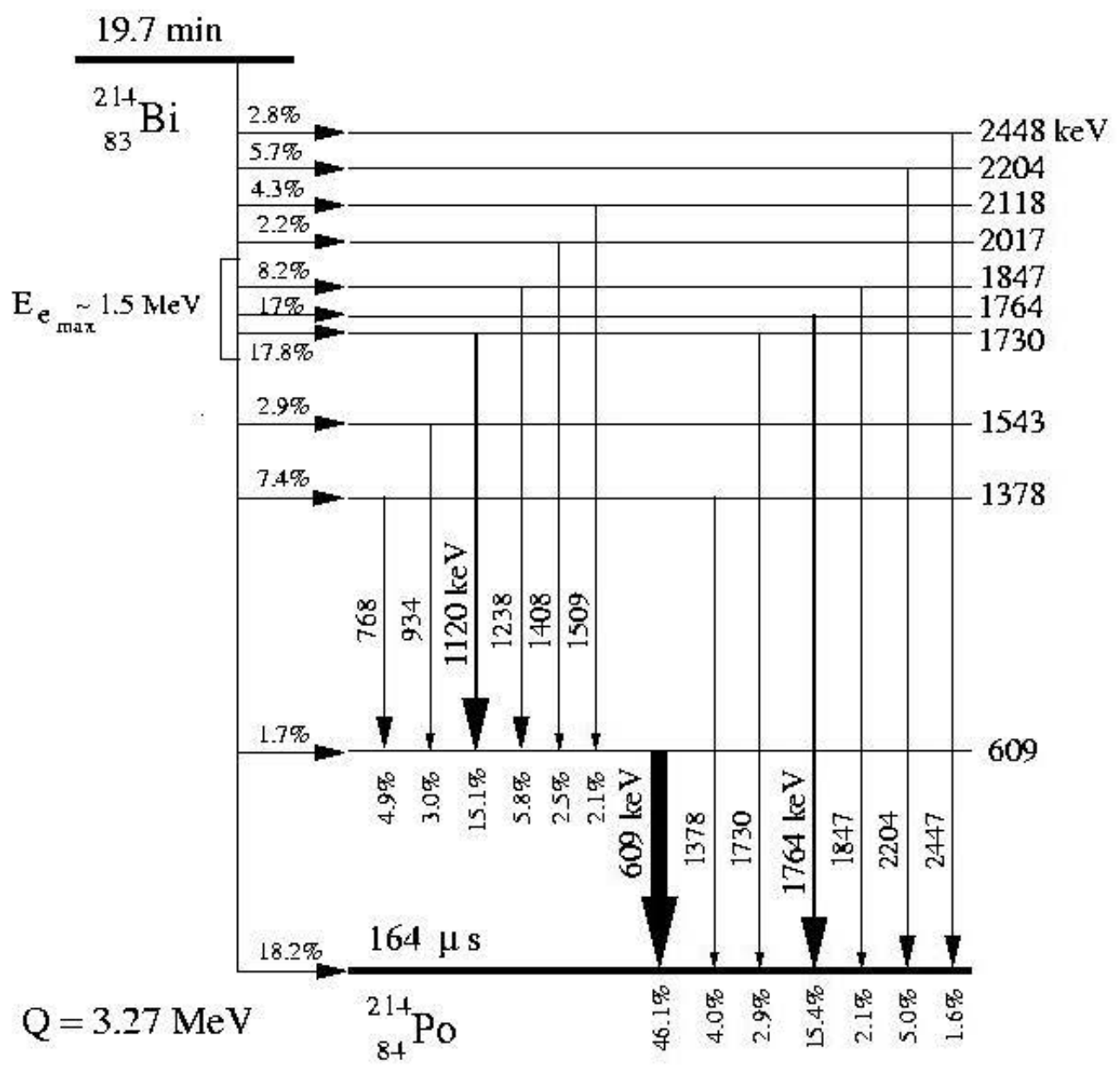

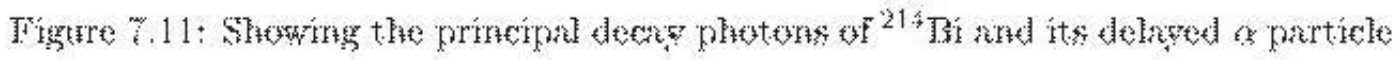
entisgitor.

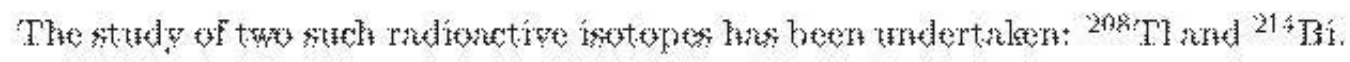

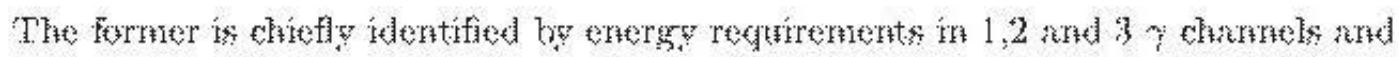

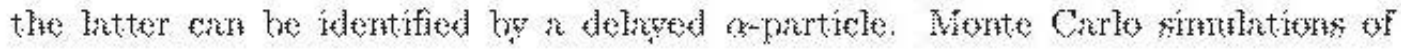

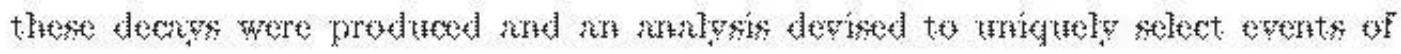

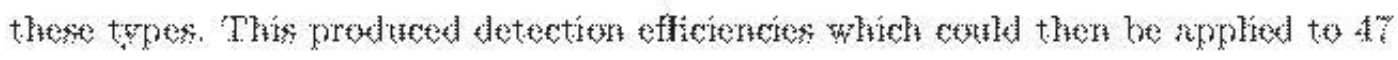
days tof real datn.

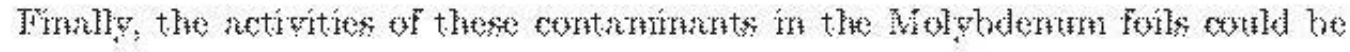
Fonfad. They were determirned to be:

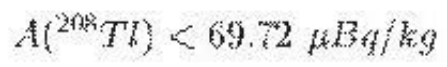



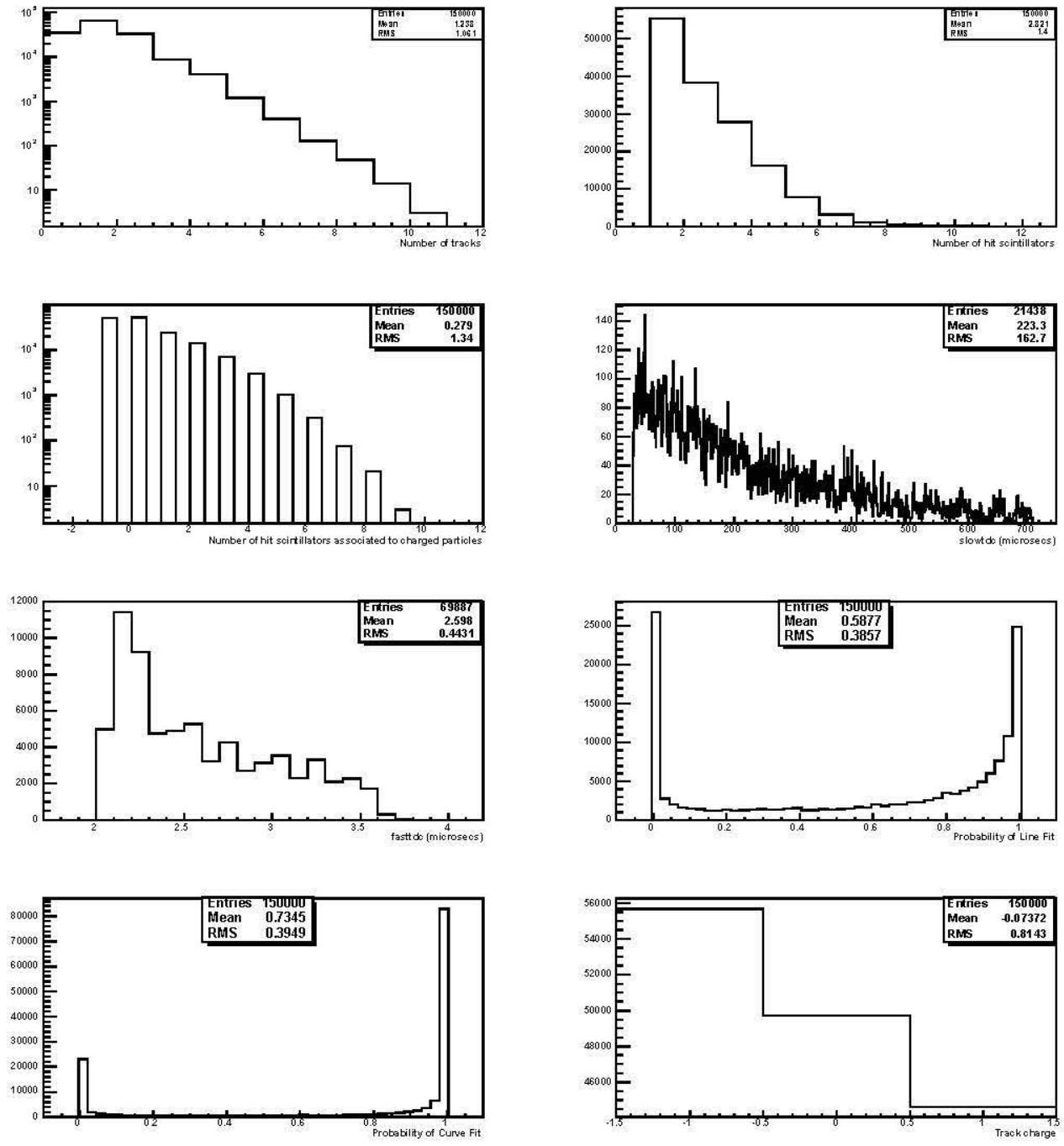

Figure 7.12: Showing the principle events quantities associated with ${ }^{214} \mathrm{Bi}$ Monte Carlo decays.

$$
A\left({ }^{214} \mathrm{Bi}\right)<274.50 \mu \mathrm{Bq} / \mathrm{kg}
$$

\subsubsection{Further Notes}

This analysis has produced results in good agreement with previous work. However it is important to note that the quoted values are upper limits only. There are a number of known second order effects that are outside the scope of this analysis. For example, the ${ }^{208} \mathrm{Tl}$ 'contamination' of the ${ }^{214} \mathrm{Bi}$ sample and vice versa could be studied. In addition, the study is incomplete without considering the activities of 

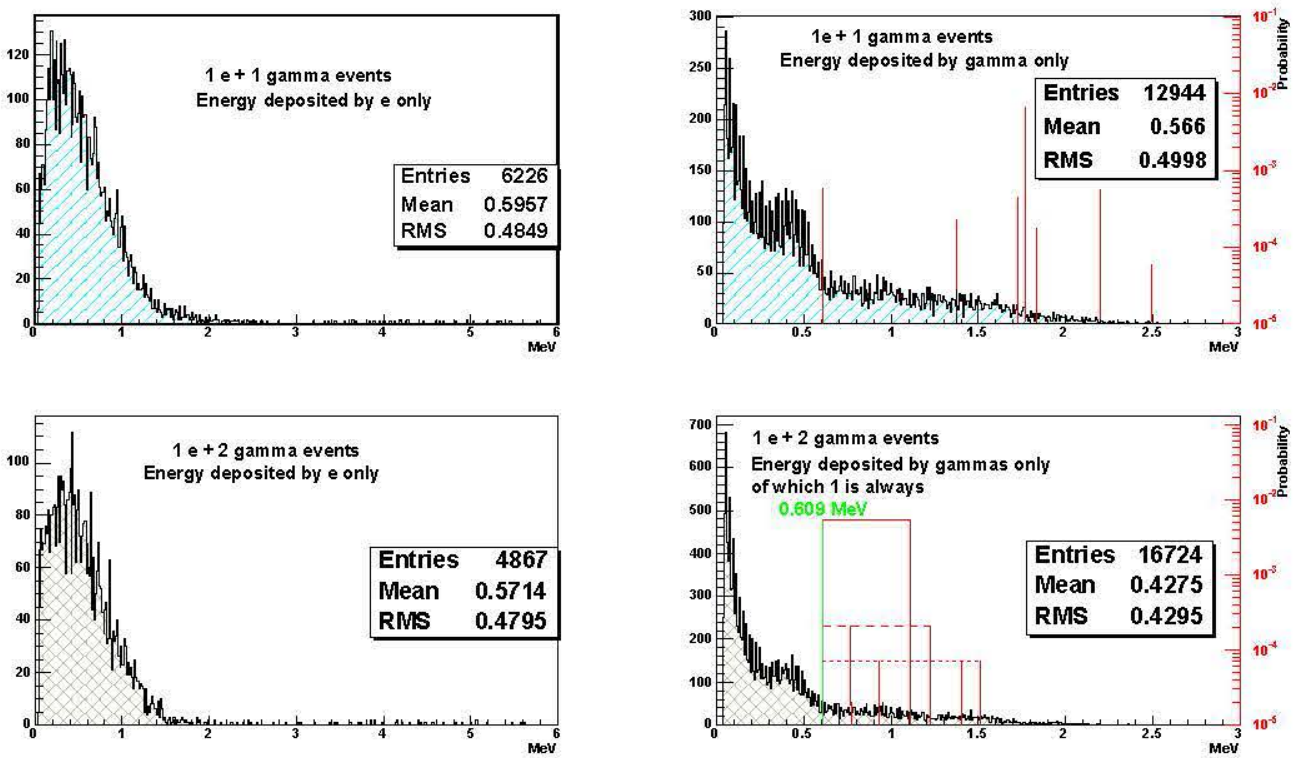

Figure 7.13: Showing the electron spectrum with one and two accompanying photons, for ${ }^{214} \mathrm{Bi}$. The probabilities of these transitions are shown on the right.

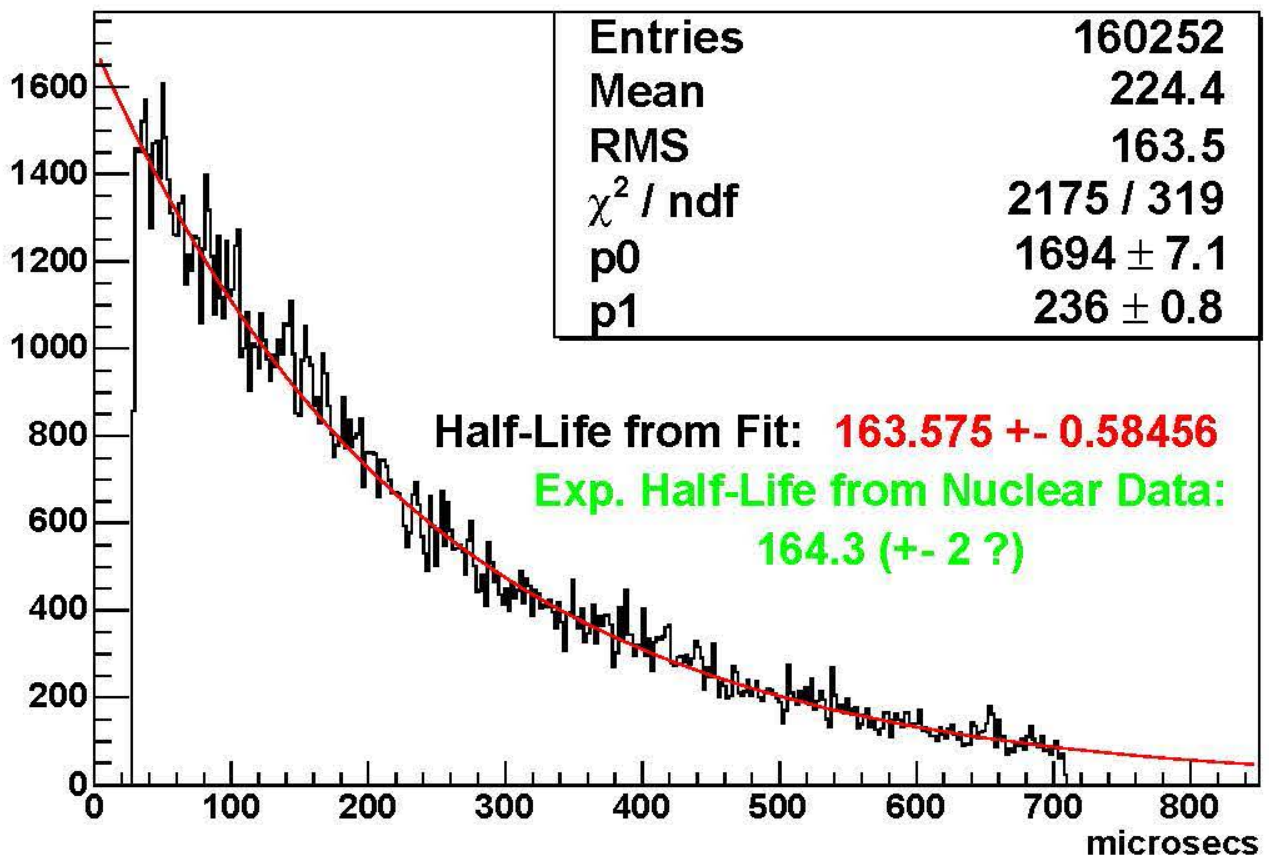

Figure 7.14: It is possible to reconstruct the half-life of the decay by plotting the times after the initial hit that the $\alpha$-particle was detected. The result is in good agreement with the expected value. 
various detector components such as the wires and PMs. It is thought that ambient radon attaches itself to the wires, increasing their activity, for example.

The activities of these isotopes are crucial numbers to understand. Their application is in the calculation of the number of events that they are expected to simulate in analysis channels (such as $0 \nu \beta \beta$ ). In this case, the analysis cuts must be applied to these background Monte Carlos to see how many unwanted events are selected. This is then multiplied by the acquisition time of the dataset and the measured activity. 


\section{Chapter 8}

\section{Conclusions}

MINOS is a long baseline neutrino oscillation experiment. It has three detector components, the Near Detector where the unoscillated spectrum is sampled, the Far Detector where the oscillated spectrum would be seen and CalDet which provided a test-bed for the electronics and methodology used, as well as providing the handle on the absolute energy scale of the experiment. Within 5 years, the MINOS experiment intends to establish whether neutrino oscillations are the cause of $\nu_{\mu}$ disappearance and if so, measure the oscillation parameters to $10 \%$.

Accurate calibration of CalDet is the first step to achieving a Near-to-Far absolute energy scale calibration. A number of calibration issues have been addressed in this work. It has been found that around 1 in 7 hits in the data can be designated as false, crosstalk hits and the effect of correctly handling these hits is to reduce the average muon range by almost $1 \%$. At CalDet, cerenkov counters can be used to remove pion contamination from the muon sample. These detectors were found to be around $85 \%$ efficient at finding on-momentum muons. Shape and energy cuts are used to separate pions and muons at the larger detectors. It has been shown that $94 \%$ of muons can be correctly identified and $97 \%$ of pions, in this way. In conjunction with the cerenkov counters, this led to the conclusion that the beamline simulation was underpredicting the number of muons present in the upstream region by up to $100 \%$. Bethe-Bloch fits to muon energy loss in CalDet was successful and agreed to a good degree with the published results from Groom et al.

NEMO-3 is a neutrinoless double beta decay experiment. Situated in the Fréjus 
tumnel between France and Italy, it houses over $8 \mathrm{~kg}$ of double beta decay isotopes. The experiment hopes to demonstrate that the neutrino is a massive, Majorana particle and can search for the effective Majorana electron neutrino mass $<m_{v}^{\epsilon}>$ down to the level of $0.1 \mathrm{eV}$. Large uncertainties in nuclear matrix element calculations introduce a large error on the final answer however.

NEMO-3 is a tracking calorimeter and it tags neutrinoless double beta events by searching for decays producing two electrons with the correct summed energy. Backgrounds to these events come from a variety of internal and external sources, most notably from radioactive contaminants of the double beta decay sources themselves. ${ }^{208} \mathrm{TI}$ and ${ }^{214} \mathrm{Bi}$ are known contaminants and events produced by these isotopes can be simulated. Applying detection efficiencies to the data, it has been found that the ${ }^{208} \mathrm{Tl}$ contamination is $<69 \mu \mathrm{Bq} / \mathrm{kg}$ in the 1,2 and 3 photon channels and $<274 \mu \mathrm{Bq} / \mathrm{kg}$ in the ${ }^{214} \mathrm{Bi}$ alpha particle channel. 


\section{Appendix A}

\section{Molybdenum Purification}

\section{Processes}

\section{A.1 ${ }^{100}$ Mo Metallic Foils}

Developed at ITEP, Moscow, this purification technique is based upon melting the molybdenum and removing the impurities. The material is melted using an electron beam and a crystal of pure material is removed with a long, narrow cylinder, leaving the impurities behind. The cylinders of metal are cropped and rolled into foils, then trimmed again. This process has yielded $2.479 \mathrm{~kg}$ of Molybdenum for the NEMO-3 experiment, which is deemed to be $95.1-98.9 \%$ pure ${ }^{100} \mathrm{Mo}$. This process guarantees the activities from ${ }^{208} \mathrm{Tl}$ and ${ }^{214} \mathrm{Bi}$ contamination to be less than $0.3 \mathrm{mBq} / \mathrm{kg}$.

\section{A.2 ${ }^{100}$ Mo Composite Foils}

The production of these foils involved a chemical procedure developed and processed at INEEL in Idaho, USA. This method specifically targets the daugter isotpes of the ${ }^{238} \mathrm{U}$ and ${ }^{232} \mathrm{Th}$ decay chains. Molybdenum metal powder is first dissolved in 4 molar $\mathrm{HNO}_{3}$ that also contains $\mathrm{Ba}\left(\mathrm{NO}_{3}\right)_{2}$. Radon present in the Molybdenum preferentially reacts with this compound. The soulution is heated to produce a slurry of $\mathrm{MoO}_{3}$ which is filtered and rinsed with ultra-pure water. It is finally reduced to a powder by heating it to $200^{\circ} \mathrm{C}$ in an inert He atmosphere. The powder is finally 
fixed to mylar strips with rhodovial, a binding paste. At the end of this process, the activities from ${ }^{208} \mathrm{Tl}$ and ${ }^{214} \mathrm{Bi}$ contamination are less than $0.14 \mathrm{mBq} / \mathrm{kg}$. 


\section{Bibliography}

[1] M. Cribier, M. Spiro, D. Vignaud, "La lumière et des neutrinos", Édition du Seuil.

[2] J. Chadwick, Nature, Feb 27, p.312, (1932).

[3] E. Fermi, Z. Physik 88, 161 (1934).

[4] Phys. Rev. 48, 512 (1935).

[5] Phys. Rev. 56, 1184 (1939).

[6] C. L. Cowen, F. Reines, et al. Science 124, 103 (1956).

[7] C. S. Wu, E. Ambler, R. W. Hayward, D. D. Hoppes, and R. P. Hudson, Phys. Rev. 105, 1413 (1957).

[8] S. M. Bilenky, B. Pontecorvo, Quark-Lepton Analagy and Neutrino Oscillations, Phys. Lett., B61, 248 (1976).

[9] URL http://nobelprize.org/physics/educational/poster/1988/hunt.html.

[10] J. N. Bahcall, R. Davis, Jr. Science 191, 264-267 (1976).

[11] K. S. Hirata et al. (Kamiokande Collaboration), Phys. Rev. Lett. 65, 1297 $(1990)$.

[12] R. Bionta et al., Observation of a Neutrino Burst in Coincidence with Supernova 1987 A in the Large Magellanic Cloud, Phys. Rev. D 37, 3361, (1987).

[13] B. Adeva et al. (L3), Z. Phys. C51, 179 (1991). 
[14] G. Alexander et al. (OPAL), Z. Phys. C52, 175 (1991).

[15] P. Abreu et al. (DELPHI), Nucl. Phys. B367, 511 (1991).

[16] D. Decamp et al. (ALEPH), Z. Phys. C53, 1 (1992).

[17] R. Barate et al., Eur. Phys. J. C. 2, 395 (1998).

[18] S. N. Ahmad et al. (SNO Collaboration), (2003),nucl-ex/0309004.

[19] Y. Ashie, et al., Evidence for an oscilliatory signature in atmospheric neutrino ascillation, Phys. Rev. Lett. 93, (2004).

[20] Q. Ho-Kim, Ph.X. Yem, Elementary Particles and Their Interactions - Concepts and Phenomena, Springer, (1998).

[21] K. Hagiwara, et al., Review of Particle Physics, Physics Review D66, 010001, (2002).

[22] S. Weinberg, The Quantum Theory of Fields Cambridge University Press, (1996).

[23] M. Rychlik, Lagrangian and Hamiltonian Mechanics, an Introduction, URL http://alamos.math.arizona.edu/ rychlik/557-dir/mechanics/

[24] P. W. Higgs, Broken Symmetries, Massless Particles and Gauge Fields, Phys. Lett. B. 12, 132 (1964)

[25] H. Leutwyler, URL arXiv.org:hep-ph/9409422 (2005-09-20).

[26] S. Weinberg, Phys. Rev. D. 5, Issue 8 (1972).

[27] R. N. Mohapatra, G. Senjanovic, Phys. Rev. D. 23, 165 (1981).

[28] Z. Maki, M. Nagakawa, S. Sakata, Prog. Theor. Phys. 28, 870 (1962).

[29] P. Adamson, et al., MINOS TDR, (MINOS Collaboration), 2004

[30] Phys. Rev. D. 17, 2369 (1978).

[31] S. P. Mikheyev, A. Yu. Smirnov, Sov. J. Nucl. Phys., 42, 913 (1985). 
[32] M. Goldhaber, L. Grodzins, and A. W. Sunyar, Phys. Rev. 109, 1015 (1958).

[33] A. Osipowicz, et al., (Katrin Collaboration), Letter of Intent, hep-ex/0109033

[34] M. Furlan, et al., Low temperature detectors for neutrinos and dark matter-III Gif-sur-Yvette, 21 (1991).

[35] K. Assamgan et al., Phys. Rev. D. 53, 6065 (1996).

[36] J. N. Bahcall, S. L. Glashow, Nature 326, 476-477 (1987).

[37] S. Hannestad, Journal of Cosmology and Astroparticle Physics 305, 4.

[38] T. Kirsten et al., Proc. Int. Symp. Nuclear Beta Decay and Neutrinos, World Scientific, 81, (1986).

[39] T. Kirsten, et al., Phys. Rev. Lett. 20, 1300 (1968).

[40] E. W. Hennecke, O. K. Manuel, and D. D. Sabu, Phys. Rev. C. 11, 1378 (1975).

[41] E. W. Hennecke, Phys. Rev. C. 17, 1168 (1978).

[42] K. Marti, and S. V. S. Murty, Phys. Rev. B. 59, 2108 (1999).

[43] B. T. Cleveland et al., Phys. Rev. Lett. 35, 757 (1975).

[44] L. Balysh et al. (Heidelberg-Moscow Collaboration), Phys. Lett. B. 322, 176 (1994).

[45] L. Baudis et al. (Heidelberg-Moscow Collaboration), Phys. Lett. B. 407, 219 (1997).

[46] M. Günther et al. (Heidelberg-Moscow Collaboration), Phys. Lett. D. 55, 54 (1997).

[47] A. M. Bakalyarov, et al. (Heidelberg-Moscow Collaboration), "Results of the experiment on investigation of Germanium-76 double beta decay. Experimental data of Heidelberg-Moscow collaboration November 1995-August 2001", Phys. Part. Nucl. Lett., 2, 77 (2005).

[48] C. E. Alseth et al. (IGEX Collaboration), Phys. Rev. C. 59, 2108 (1999). 
[49] C. E. Alseth et al. (IGEX Collaboration), Phys. At. Nucl. 63, 1225 (2000).

[50] I. G. Irastorza, A. Morales, and S. Scopel,URL hep-ph/0108146.

[51] R. Ardito, et al. (CUORE Collaboration), URL hep-ex/0501010, (2005).

[52] A. I. Abazov et al. (SAGE Collaboration), Phys. Rev. Lett. 67, 3332 (1991).

[53] D. N. Abdurashitov et al. (SAGE Collaboration), Phys. Rev. Lett. 77, 4708 (1996).

[54] P. Anselmann et al. (GALLEX Collaboration), Phys. Lett. B. 285, 376 (1992)

[55] W. Hampel et al. (GALLEX Collaboration), Phys. Lett. B. 388, 384 (1996)

[56] M. Altmann et al. (GNO Collaboration), Phys. Lett. B. 490, 16 (2000)

[57] Y. Fukadaa et al.(Super-K Collaboration), Phys. Rev. Lett. 81, 1562 (1998).

[58] M. Aglietta et al., Europhys. Lett. 8, 611 (1989).

[59] K. Daum et al., Z. Phys. C. 66, 417 (1995).

[60] W. W. M. Allison et al., Phys. Lett. B. 449, 137 (1999).

[61] M. Ambrosio et al. (MACRO collaboration), Eur. Phys. J, 323 (2004).

[62] D. Duchesneau, (OPERA Collaboration), 2002, hep-ex/0209082

[63] R. Arnold, et al. (NEMO Collaboration), "First Results of the Search for Neutrinoless Double-Beta Decay with the NEMO-3 Detector", Phys. Rev. Lett., 2 , 77 (2005).

[64] M. Appollonio et al.(CHOOZ collaboration), Phys. Lett. B. 95, 28 Oct (1999).

[65] K. Eguchi et al.(KamLAND collaboration), Phys. Rev. Lett. 90, 021802 (2003).

[66] M. Maltoni, T. Schwetz, J. W. F. Valle, and M. A. Tortola,hep-ph/0405172.

[67] Y. Fukada et al. (Super-K Collaboration), Phys. Rev. Lett. 82, 1810 (1999).

[68] S. Fukada et al. (Super-K Collaboration), Phys. Rev. Lett. 86, 5656 (2001). 
[69] T. J. Haines et al. (IMB Collaboration), Phys. Rev. Lett. 57, 1986 (1986).

[70] L. Ludovici, Nucl. Phys. Proc. Suppl, 91, 177 (2001).

[71] P. Astier et al. (NOMAD Collaboration), Nucl. Phys. B. 611, 3 (2001).

[72] P. Adamson et al., Nucl. Instrum. Meth. A492, 325 (2002).

[73] C. Athanassopoulos et al. (LSND Collaboration), Phys. Rev. Lett., 81, 1774 (1988).

[74] R. Arnold et al. (NEMO Collaboration), Nucl. Instr, and Meth. in Phys. Res. A., (2003).

[75] K. Nakamura (K2K Collaboration), Nucl. Phys. Proc. Suppl. 91, 203 (2001).

[76] M. H. Ahn et al. (K2K Collaboration), Phys. Rev. Lett. 90, 041801 (2003).

[77] D. Groom, Atomic and Nuclear Properties of Materials for 292 substances. URL http://durpdg.dur.ac.uk/lbl/AtomicNuclearProperties/

[78] R. Arnold, V. Tretiak, NEMO-3 simulation program - User's Guide, IReS1/2001, December 2001).

[79] P. Adamson, "Crosstalk Measurements in M16 at Sussex.", NuMI-NOTE-0622.

[80] J. Day, S. Fuqua, M. Kordosky, K. Lang, P. Vahle, A. Winbow, "Results from Comprehensive testing of an Evaluation Sample of 50 R5900-00-M16 PMTs for the MINOS detector:", NuMI-NOTE-0663.

[81] J. Liu et al., "Basic Cross-Talk Analysis of the Data from the M16 Test Stands." NuMI-NOTE-0735.

[82] R. Lee, S. Seun, "Crosstalk Measurements at the Far Detector:", NuMI-NOTE0795 .

[83] L. Jenner, URL http://www.hep.ucl.ac.uk/ lj/minos 26 .htm.

[84] R. Nichol, C. Smith, "Strip-to-Strip Calibration at the Calibration Detector:", NuMI-NOTE-0972. 
[85] R. Nichol, C. Smith, "Procedure for the Relative Calibration of the Calibration. Detector.", NuMI-NOTE-0973.

[86] A. Cabrera, "Studies of Crosstalk on the MINOS" Hamamatsu M64 Photomultipliers.", NuMI-NOTE-0976.

[87] M. Barker, A. De Santo, P. Dervan, K. Lang, P. Miyagawa, R. Saakyan, C. Smith, D. Michael, J. Thomas, A. Weber, "Testing of Hamamatsu R590000-M64 Multi-Pixel PMTs for MINOS.", NuMI-NOTE-0820.

[88] A. Cabrera, A. De Santo, P. Miyagawa, N. Tagg, A. Weber, "Hamamatsu R5900-00-M64 PMT Characteristics Measured by the Oxford Test Stand.", NuMI-NOTE-0934.

[89] L. Jenner, URL http://www.hep.ucl.ac.uk/ 1j/minos 21 .htm.

[90] P. Adamson, A. Para, J. Thomas, "Performance of a least-squares based muon. reconstruction procedure for MINOS.", NuMI-NOTE-SIM-0536.

[91] R. Arnold et al. (Nemo Collaboration), Nucl. Inst. Meth. A536, 79-122 (2005). 